AperTO - Archivio Istituzionale Open Access dell'Università di Torino

\title{
On some aspects of oscillation theory and geometry
}

\section{This is the author's manuscript}

Original Citation:

Availability:

This version is available http://hdl.handle.net/2318/1693259

since 2019-02-18T20:59:20Z

Published version:

DOI:10.1090/S0065-9266-2012-00681-2

Terms of use:

Open Access

Anyone can freely access the full text of works made available as "Open Access". Works made available under a Creative Commons license can be used according to the terms and conditions of said license. Use of all other works requires consent of the right holder (author or publisher) if not exempted from copyright protection by the applicable law. 


\title{
On some aspects of Oscillation Theory and Geometry
}

\author{
Bruno Bianchini \\ Luciano Mari \\ Marco Rigoli
}

Author address:

Dipartimento di Matematica Pura ed Applicata, Università degli Studi di Padova, Via Trieste 63, I-35121 Padova, ITALY

E-mail address: bianchini@dmsa.unipd.it

Dipartimento di Matematica, Università degli Studi di Milano, Via SALDini 50, I-20133 Milano, ITALY

E-mail address: lucio.mari@libero.it

Dipartimento di Matematica, Università degli Studi di Milano, Via SAldini 50, I-20133 Milano, ITALY

E-mail address: marco.rigoli@unimi.it, marco.rigoli55@gmail.com 



\section{Contents}

Introduction $\quad \mathrm{v}$

Chapter 1. The Geometric setting 1

1.1. Cut-locus and volume growth function 1

1.2. Model manifolds and basic comparisons $\quad 10$

1.3. Some spectral theory on manifolds 23

Chapter 2. Some geometric examples related to oscillation theory 35

2.1. Conjugate points and Myers type compactness results 35

2.2. The spectrum of the Laplacian on complete manifolds 43

2.3. Spectral estimates and immersions 50

2.4. Spectral estimates and nonlinear PDE 54

Chapter 3. On the solutions of the ODE $\left(v z^{\prime}\right)^{\prime}+A v z=0 \quad 63$

3.1. Existence, uniqueness and the behaviour of zeroes 63

3.2. The critical curve: definition and main estimates 68

$\begin{array}{lll}\text { Chapter } 4 . & \text { Below the critical curve } & 81\end{array}$

4.1. Positivity and estimates from below 81

4.2. Stability, index of $-\Delta-q(x)$ and the uncertainty principle $\quad 87$

4.3. A comparison at infinity for nonlinear PDE $\quad 95$

4.4. Yamabe type equations with a sign-changing nonlinearity 102

4.5. Upper bounds for the number of zeroes of $z \quad 110$

$\begin{array}{lll}\text { Chapter 5. Exceeding the critical curve } & 115\end{array}$

5.1. First zero and oscillation $\quad 115$

5.2. Comparison with known criteria 121

5.3. Instability and index of $-\Delta-q(x) \quad 124$

5.4. Some remarks on minimal surfaces 126

5.5. Newton operators, unstable hypersurfaces and the Gauss map 134

$\begin{array}{ll}\text { 5.6. Dealing with a possibly negative potential } & 145\end{array}$

5.7. An extension of Calabi compactness criterion 147

$\begin{array}{lll}\text { Chapter 6. } & \text { Much above the critical curve } & 157\end{array}$

6.1. Controlling the oscillation 157

6.2. The growth of the index of $-\Delta-q(x) \quad 172$

6.3. The essential spectrum of $-\Delta$ and punctured manifolds 173

$\begin{array}{ll}\text { Bibliography } & 181\end{array}$ 


\begin{abstract}
The aim of this paper is to analyze some of the relationships between oscillation theory for linear ordinary differential equations on the real line (shortly, ODE) and the geometry of complete Riemannian manifolds. With this motivation we prove some new results in both directions, ranging from oscillation and nonoscillation conditions for ODE's that improve on classical criteria, to estimates in the spectral theory of some geometric differential operator on Riemannian manifolds with related topological and geometric applications. To keep our investigation basically self-contained we also collect some, more or less known, material which often appears in the literature in various forms and for which we give, in some instances, new proofs according to our specific point of view.
\end{abstract}

Received by the editor February 24, 2011.

2010 Mathematics Subject Classification. Primary 34K11, 58C40, 35J15; Secondary 35J10, $53 \mathrm{C} 21,57 \mathrm{R} 42$.

Key words and phrases. oscillation, spectral theory, index, Schrodinger operator, uncertainty principle, compactness, immersions, comparison. 


\section{Introduction}

Ordinary Differential Equation (hereafter, ODE) techniques are a powerful tool in investigating the geometry of a complete Riemannian manifold $(M,\langle\rangle$,$) , and$ their importance can be hardly overestimated. For instance, the classical comparison and oscillation theory for $g^{\prime \prime}-G g=0$ is fruitful in the investigation of Jacobi fields and related Hessian, Laplacian and volume comparison theorems for $M$, and to obtain sharp extensions of the classical Bonnet-Myers compactness theorem (in this respect, see $[\mathbf{G a l 8 2}]$, [Kup86], [EO80]). As a second example, radialization techniques lead in favourable circumstances to the study of an ordinary differential equation to control the solutions of a given partial differential equation. In both instances, the study of the sign of the solutions of the ODE, and the positioning of the possible zeros, reveals to be one of the challenging problems involved. In our work, we will be concerned with a solution $z(r)$ of the following Cauchy problem:

$$
\left\{\begin{array}{l}
\left(v(r) z^{\prime}(r)\right)^{\prime}+A(r) v(r) z(r)=0 \quad \text { on } \mathbb{R}^{+} \\
z^{\prime}(r)=O(1) \quad \text { as } r \downarrow 0^{+} \quad, \quad z\left(0^{+}\right)=z_{0}>0
\end{array}\right.
$$

where $\mathbb{R}^{+}=(0,+\infty), v(r)$ is a non-negative function and $A(r)$ is possibly somewhere negative but in a controlled way as we shall explain at due time. The application of these results to the geometric problems we shall consider below leads us to the following requests:

$$
\begin{aligned}
& A(r) \in L_{\mathrm{loc}}^{\infty}\left(\mathbb{R}_{0}^{+}\right), \quad \text { where } \mathbb{R}_{0}^{+}=[0,+\infty), \\
& 0 \leq v(r) \in L_{\mathrm{loc}}^{\infty}\left(\mathbb{R}_{0}^{+}\right) \quad, \quad 1 / v(r) \in L_{\mathrm{loc}}^{\infty}\left(\mathbb{R}^{+}\right) \\
& v(r) \text { is non decreasing near } 0 \text { and } \lim _{r \rightarrow 0^{+}} v(r)=0 .
\end{aligned}
$$

For our purposes we shall look for solutions $z(r) \in \operatorname{Lip}_{\text {loc }}\left(\mathbb{R}_{0}^{+}\right)$, that is, locally Lipschitz solutions. For the sake of completeness, in Section 3.1 we supply the basic ODE material related to $(C P)$. To illustrate a typical framework where the study of the solutions $z$ of $(C P)$ reveals to be useful, we consider on $M$ a Schrödinger operator of the type $L=-\Delta-q(x)$, where $q(x) \in L_{\mathrm{loc}}^{\infty}(M)$, and we search for estimates for the bottom of the spectrum of $L, \lambda_{1}^{L}(M)$, or for the index of $L, \operatorname{ind}_{L}(M)$. The key problem is to discover the critical growth of $q(x)$ that discriminates between the various cases that may occur: clearly, this critical growth must only depend on the geometry of $M$. Towards this purpose, to have a first insight into the matter we "radialize" the problem. Suppose that we want to prove, under suitable conditions on $q$, that $\lambda_{1}^{L}(M) \geq 0$ or $\operatorname{ind}_{L}(M)<+\infty$. By Theorems 1.33 and 1.41 below, it is enough to produce a positive, weak solution $u$ of $\Delta u+q(x) u \leq 0$ on $M$ or outside some compact set. Suppose for convenience that we are on a model manifold $\left(M_{g}, \mathrm{~d} s^{2}\right)$ (see Definition 1.16 below), with metric 
given, in polar geodesic coordinates, by $\mathrm{d} s^{2}=\mathrm{d} r^{2}+g(r)^{2} \mathrm{~d} \theta^{2}$, and let $A$ be a continuous, non-negative function such that $q(x) \leq A(r(x))$. Then, if we search $u$ of the form $u(x)=z(r(x))$, the problem shifts to the search of a positive solution $z$ (say $C^{1}$ ) of the ODE

$$
z^{\prime \prime}+(m-1) \frac{g^{\prime}}{g} z^{\prime}+A z=0 \quad \text { on } I=\left[r_{0},+\infty\right), r_{0} \geq 0 .
$$

Multiplying by the model volume density $g^{m-1}$, this can be rewritten as the SturmLiouville equation

$$
\left(g^{m-1} z^{\prime}\right)^{\prime}+A g^{m-1} z=0 .
$$

As we will see in this paper, we shall require the initial conditions $z\left(r_{0}\right)=z_{0}>0$, $z^{\prime}\left(r_{0}\right)=0$ in order to match with the inequalities of the Laplacian comparison theorem when we will deal with non-radial manifolds. Therefore, this leads to investigate the qualitative properties of the solution of $(C P)$ with $v=g^{m-1}$. If $A$ is sufficiently small, then $z$ is positive on $\left[r_{0},+\infty\right)$. With the aid of some spectral results that we shall recall in Section 1.3 , we can infer that $\lambda_{1}^{L}(M) \geq 0$ (when $r_{0}=0$ ), or that $\operatorname{ind}_{L}(M)<+\infty$ (when $r_{0}>0$ ). Suppose now that $r_{0}=0$ and $q(x) \geq A(r(x))$. If $z$ has a first zero at some $R$, then $u$ solves

$$
\left\{\begin{array}{l}
-L u=\Delta u+q u \geq 0 \text { on } B_{R}, \\
u=0 \text { on } \partial B_{R} .
\end{array}\right.
$$

By a simple argument, $\lambda_{1}^{L}(M)<0$. Indeed, by contradiction, if $\lambda_{1}^{L}(M) \geq 0$ then by the monotonicity of eigenvalues $\lambda_{1}^{L}\left(B_{R}\right)>0$. Let $0<w$ be the first eigenfunction of $L$ on $B_{R}$ with Dirichlet boundary conditions, that is, $w$ solves $L w=\lambda_{1}^{L}\left(B_{R}\right) w$ on $B_{R}, w=0$ on $\partial B_{R}$. Then, integrating by parts,

$$
0>-\lambda_{1}^{L}\left(B_{R}\right) \int_{B_{R}} u w=\int_{B_{R}} u(\Delta w+q w)=\int_{B_{R}} w(\Delta u+q u) \geq 0,
$$

a contradiction. Similarly, if $z$ oscillates, for every $r_{0}>0$ we can choose two consecutive zeroes $R_{1}<R_{2}$ of $z$ after $r_{0}$. Then, $u(x)=z(r(x)) \chi_{B_{R_{2}} \backslash \bar{B}_{R_{1}}}(x)$ solves $\Delta u+q u \geq 0$ on the annulus $B_{R_{2}} \backslash \bar{B}_{R_{1}}$, with zero boundary conditions. The above argument leads to $\lambda_{1}^{L}\left(M \backslash B_{r_{0}}\right)<0$, so $\operatorname{ind}_{L}(M)=+\infty$ again by Theorem 1.41. As a matter of fact, both the negativity of $\lambda_{1}^{L}(M)$ and $\operatorname{ind}_{L}(M)=+\infty$ can be obtained via radialization on each complete, non-compact Riemannian manifold by means of the Rayleigh characterization. The idea is as follows: let $v(r)$ be the volume of $\partial B_{r}$. By Proposition 1.6 below, in general we can only assume that $v$ is locally bounded, and bounded away from zero on compact subsets of $\mathbb{R}^{+}$. Suppose that the problem $(C P)$ admits a solution $z \in \operatorname{Lip}_{\text {loc }}\left(\mathbb{R}_{0}^{+}\right)$with a first zero $R$. Then, integrating by parts, the test function $\phi(x)=z(r(x)) \chi_{B_{R}}(x)$ solves

$$
\int_{B_{R}}|\nabla \phi|^{2}-A \phi^{2}=-\int_{0}^{R}\left[\left(v(s) z^{\prime}(s)\right)^{\prime}+A(s) v(s) z(s)\right] z(s) \mathrm{d} s=0,
$$

whence $\lambda_{1}^{L}(M)<0$ by the min-max characterization and the monotonicity of eigenvalues. Analogous computation shows that $\operatorname{ind}_{L}(M)=+\infty$ provided $z$ oscillates. This shows how spectral problems on $M$ can be related to the central theme of our ODE investigation. 
Developing ideas in [BR97] and [BMR09], the core of all of our ODE results lies in the identification of an explicit critical curve $\chi(r)$, depending only on $v(r)$, and which gives the border line for the behavior of $z(r)$. Roughly speaking and considering the simplest case $A(r) \geq 0$, if $A(r)$ is much greater than $\chi(r)$ in some region, then $z(r)$ has a first zero, while if $A(r)$ is not larger than $\chi(r)$ solutions are positive on $\mathbb{R}_{0}^{+}=[0,+\infty)$ and explicit lower bounds are provided. Using the critical curve we will be able to obtain sharp conditions on $A$ for the existence and localization of a first zero of $z$, and for the oscillatory behavior of $z$. Furthermore, the key technical ODE result of the paper will enable us to estimates the distance between two consecutive zeros of an oscillatory solution $z$ of $(C P)$ under very general assumptions.

Besides the estimates on the spectrum of Schrödinger operators just described, the ODE techniques that we are going to develop will enable us to get bounds from above on the growth of the spectral radius of the Laplacian outside geodesic balls, even when the volume growth of the manifold is faster than exponential. The spectral results that we shall obtain, in turn, have many geometric applications in the setting of minimal and higher order constant mean curvature hypersurfaces of $\mathbb{R}^{m}$, their Gauss map, minimal surfaces and the Yamabe problem, and so on. For more information, we refer to the description of the contents of the various chapters that we shall present in a while.

Another geometric application deserves particular attention. Indeed, in a quite simple way our results on solutions $z$ of $(C P)$ can be used to get sharp extensions of previous compactness criteria for complete manifolds, in the spirit of the BonnetMyers theorem mentioned at the beginning of this introduction. For this reason, throughout the paper we will often shift our attention from one another of the problems

$$
\begin{aligned}
& \text { (1) }\left\{\begin{array}{l}
\left(v z^{\prime}\right)^{\prime}+A v z=0 \quad \text { on } \mathbb{R}^{+}, \\
z(0)=z_{0}
\end{array}\right. \\
& \text { (2) }\left\{\begin{array}{l}
g^{\prime \prime}-G g=0 \\
g(0)=0, \quad g^{\prime}(0)=1,
\end{array} \text { on } \mathbb{R}^{+},\right.
\end{aligned}
$$

or of their counterparts with initial condition at some $r_{0}>0$. According to the situation, properties that we will establish for (1) will be successively rephrased for (2), or viceversa. More precisely, we will pass from one ODE to the other in two different ways. The first is classical and widely exploited in literature, see [Lei50] and [Moo55], while (at least to our knowledge) the second has not been so much considered. For instance, as we will see, this latter substitution will be the key to prove the theorems of Chapter 4. Even more, comparisons between the two ways will lead to interesting improvements of oscillation and nonoscillation criteria for $g^{\prime \prime}-G g=0$, such as those of E. Hille and Z. Nehari, in various directions. The main geometric achievement, however, will be the extension of Calabi compactness criterion for complete manifolds, [Cal67], to the case when the Ricci curvature along geodesics $\gamma(r)$ emanating from some origin is bounded by $-B^{2} r^{\alpha}$ on $\left[r_{0},+\infty\right)$, for some $r_{0}>0, B \geq 0$ and $\alpha \geq-2$, improving on all of the results in the most recent literature. 
In an attempt to give a unified approach to a number of apparently different geometric problems, based on the notion of critical curve, the paper is organized as follows.

In Chapter 1 we collect and prove some facts on the cut-locus of a point (or more generally of a submanifold) and on the behaviour of the function $\operatorname{vol}\left(\partial B_{r}\right)$ that shall determine the regularity of the coefficients in the Cauchy problem $(C P)$. We then prove some basic geometric comparison results such as the Laplacian and the Hessian comparison theorems. Their proofs will be accomplished starting from the Ricci commutation rules for third covariant derivative, without the use of Jacobi fields. The chapter ends with a short review of spectral theory on manifolds. We give a full proof of some of the most important results for our investigation, concentrating on those that, at least to our knowledge, are difficult to find in book form.

Chapter 2 describes a number of geometric examples that are related to oscillation theory, with the purpose to show the reader instances of the interaction of this latter with geometry. First, we discuss the relation between conjugate points and compactness results for complete manifolds beginning with the original theorem of Myers and proceeding with its more recent generalizations, including the well known cornerstone of Calabi. As a matter of fact, we extend the discussion to the case when the Ricci curvature is bounded below by a negative constant. In the subsequent section we collect and prove a number of, by now classical, theorems on the spectrum of the Laplacian on manifolds with a pole. Besides providing the necessary background for non-specialists, these help putting some results of Chapters 4 and 6 in perspective. We then present a mild extension of a very recent result of Bessa, Jorge and Montenegro [BJM10], which positively answers a question of S.T. Yau on the discreteness of the spectrum of the Martin-Morales-Nadirashvili minimal surface in $\mathbb{R}^{3}$. In the final part of the chapter we illustrate the use of spectral estimates in establishing the existence of positive solutions to Yamabe-type equations on a complete manifold, that is, equations of the form

$$
\Delta u+q(x) u-b(x) u^{\sigma}=0, \quad q(x), b(x) \in C^{0}(M), \quad \sigma>1 .
$$

The first part of Chapter 3 is devoted to the analytical results on $(C P)$ mentioned above. These include existence and uniqueness of solutions $z \in \operatorname{Lip}_{\text {loc }}\left(\mathbb{R}_{0}^{+}\right)$, and a proof that the zeroes of $z(r)$, if any, are attained at isolated point of $\mathbb{R}^{+}$. Next, we introduce the critical curve $\chi(r)$. We provide examples of $\chi(r)$, for instance in Euclidean and hyperbolic space, discussing some of its features. Monotonicity, comparison properties, and upper and lower bounds for $\chi$ are then proved in terms of curvature requirements on the manifold. To relax geometric assumptions in subsequent sections of the paper, we also introduce the related modified curves $\chi_{f}(r)$, where $f$ is some bound for $v$, and $\tilde{\chi}(r)$.

Chapters 4, 5, 6 are the core of the paper. Here we present either brand new results or new techniques to prove known facts. In Chapter 4 we investigate the consequences of lying below the critical curve. With this we mean that the potential $A(r)$ in the linear term is smaller than the critical curve. In this situation solutions of $(C P)$ have definite sign on $\mathbb{R}^{+}$and we provide a lower bound estimate which is sharp at infinity. As we explained before, these results are then used to obtain sufficient conditions to guarantee that Schrödinger type operators $L$ have nonnegative first eigenvalue or finite index, see for instance Theorem 4.10. In the same 
vein we prove a version of the Uncertainty Principle Lemma and lower bounds on $\lambda_{1}^{L}\left(B_{R}\right), \lambda_{1}^{L}(M)$ and $\inf \sigma_{\text {ess }}(L)$ (that is, the infimum of the essential spectrum of $L$ ) on each manifold with a pole. We conclude the chapter with some applications. The first is a comparison result for non-negative sub and supersolutions of Yamabe-type equations. As a consequence, we characterize isometries in the group of conformal diffeomorphisms of a complete manifold in itself. Finally, in the last section we relate a very recent upper bound for the number of zeroes of a nontrivial solution $z(r)$ of $(C P)$ (see [EFK11]) to the critical curve. In doing so, it will be apparent that $\chi$ is also deeply linked to Hardy-Sobolev inequalities on $\mathbb{R}^{+}$. We mention that throughout the chapter we discuss, with a number of examples, the mutual relationship between the critical curves $\chi$ and $\tilde{\chi}$.

In Chapter 5 we consider the case when the potential $A(r)$ exceeds, in an integral sense, the critical curve $\chi$ or the curve $\chi_{f}$. First we establish a first zero and an oscillation criterion, both in terms of the reciprocal "integral" behaviour of $A$ and $\chi$, and we compare them with well known criteria in the literature such as those of Leighton, Moore, Hille-Nehari, Calabi and others. Then we apply our achievements to determine instability and index of Schrödinger operators. We devote the second part of the chapter to applications to geometrical problems related to minimal surfaces, higher order constant mean curvature hypersurfaces of $\mathbb{R}^{m+1}$, the distribution of their spherical Gauss map in $\mathbb{S}^{m}$ together with an interesting reduction of codimension theorem. In the last two sections, we describe a simple method to extend Calabi compactness criterion to the case of a controlled negative bound of the Ricci curvature. For its versatility, this method can be also applied to obtain sharp refinements of Calabi and Hille-Nehari oscillation criteria. A number of remarks and observations spread throughout the chapter show the sharpness of our results.

In Chapter 6 we deal with the case when $A(r)$ is much above the critical curve in a pointwise sense, and we focus our attention on the problem of determining an upper bound for the difference between two consecutive zeroes of an oscillating solution of $(C P)$. With an example we show that in order to use classical Sturm type arguments to reach the desired conclusion we need the full knowledge of the asymptotic behaviour of $v(r)$. This is, interpreting $v(r)=\operatorname{vol}\left(\partial B_{r}\right)$, a strong geometric requirement and it forces to detect a new approach to deal with the case in which our geometric information only provide an upper bound for $v(r)$. The key technical tool of this chapter is Theorem 6.5: denoting with $R_{1}(\varrho)<R_{2}(\varrho)$ the first two consecutive zeros of $z(r)$ after $r=\varrho$, we can estimate the difference $R_{2}(\varrho)-R_{1}(\varrho)$. If $v(r) \leq \exp \left\{a r^{\alpha} \log ^{\beta} r\right\}, a, \alpha>0, \beta \geq 0$, this yields

$$
R_{2}(\varrho)-R_{1}(\varrho)=O(\varrho) \quad \text { as } \varrho \rightarrow+\infty,
$$

and even more, we provide an upper estimate for

$$
\limsup _{\varrho \rightarrow+\infty} \frac{R_{2}(\varrho)}{\varrho}
$$

with an explicit constant. Further specializations of this result yield a lower bound for the growth of the index of Schrödinger type operators and an upper bound for the growth of the first eigenvalue of the Laplacian on the punctured manifold $M \backslash B_{R}$ extending, in this latter case, some results of Do Carmo and Zhou [CZ99] and Brooks [Bro81]. Again, throughout the chapter attention is paid to compare with the previous literature and to show the sharpness of our results with the aid 
of suitable counterexamples.

Acknowledgements: the authors are deeply grateful to Proff. S. Pigola and A.G. Setti for having suggested them the paper [ER11], which leads to a definite improvement of the results of Section 5.4. Furthermore, they wish to express their gratitude to prof. M. L. Leite and to the referee for helpful comments and corrections, in particular regarding Section 5.5. 


\section{CHAPTER 1}

\section{The Geometric setting}

The aim of this chapter is to introduce some basic, but sometimes not widely known, material of Riemannian geometry that shall be needed in the rest of the paper. We briefly describe the cut-locus of a submanifold $K$, recalling its main properties especially relative to the distance function from $K$. For instance, we deal with the regularity of $v(r)=\operatorname{vol}\left(\partial B_{r}\right)$, where $B_{r}$ is the set of points whose distance from $K$ is less than $r$. We then introduce some comparison procedures to estimate from above and/or below Hess $r$ and $\Delta r$, and we conclude the chapter with a short review of spectral theory on manifolds. Although most of the material covered by this chapter is somehow standard, part of each section, at least to our knowledge, is still not accessible in book form. Furthermore, in some cases a different (and we hope clearer) presentation of known results is provided. The main theorems of each section will be extensively used throughout the paper.

\subsection{Cut-locus and volume growth function}

Let $(M,\langle\rangle$,$) be a connected, complete Riemannian manifold of dimension m \geq$ 2 with induced distance function $d: M \times M \rightarrow \mathbb{R}_{0}^{+}$, and let $K \subset M$ be a properly embedded submanifold. We write $d_{K}(x)$ for the distance function from $K$, and we denote with $\partial B_{r}$ the geodesic sphere centered at $K$, that is

$$
\partial B_{r}=\left\{x \in M: d_{K}(x)=r\right\} .
$$

This introductory section deals with the regularity of the volume growth function

$$
r \longmapsto \operatorname{vol}\left(\partial B_{r}\right),
$$

where vol stands for the $(m-1)$-dimensional Hausdorff measure (see [EG92]). Although in the next chapters we will be always concerned with the case $K=\{o\}$, $o \in M$, all that we say in this section holds for any $K$. The analysis of the volume growth function is deeply related to the topology and the geometry of the cut-locus of $K$, $\operatorname{cut}(K)$. For convenience, we briefly recall the definitions and main results on $\operatorname{cut}(K)$, and we refer the reader to [GHL90] and [Sak96] for the general treatment, and to [MM03] for the study of $\operatorname{cut}(K)$ when $K$ has a lower regularity. We set $\pi: N_{K} \rightarrow K$ and $\pi: U_{K} \rightarrow K$, respectively, for the normal bundle and unit normal bundle over $K$, and let exp : $N_{K} \rightarrow M$ be the normal exponential map. Since $M$ is complete and $K$ is closed in $M$, for every $x \in M \backslash K$ there exists at least one minimizing geodesic from $K$ to $x$, and every minimizing geodesic is orthogonal to $K$, that is, it is of the form $\exp (t v)$ for some $v \in U_{K}, t \in \mathbb{R}$. For every $v \in U_{K}$, let $\gamma_{v}(s)=\exp (s v)$ be the unit speed geodesic starting from $K$ with tangent vector $v$. 
We say that $\gamma_{v}$ is a segment on $[0, t]$ if it is length minimizing on $[0, t]$. Define

$$
\begin{aligned}
& \rho(v)=\sup \left\{t>0: \gamma_{v} \text { is a segment on }[0, t]\right\} \leq+\infty \\
& \lambda(v)=\min \left\{t>0: \gamma_{v}(t) \text { is a focal point of } K \text { along } \gamma_{v}\right\} \leq+\infty .
\end{aligned}
$$

We recall that $q=\gamma_{v}(t)$ is focal for $K$ along $\gamma_{v}$ if exp is not invertible at $t v$. If $\rho(v)=+\infty, \gamma_{v}$ is called a ray. If $\rho(v)<+\infty, \exp (\rho(v) v)$ is called the cut-point of $K$ along $\gamma_{v}$, and, if $\lambda(v)<+\infty$, $\exp (\lambda(v) v)$ is the first focal point of $K$ along $\gamma_{v}$. If $q=\gamma_{v}(t)$ is a focal point of $K$ along $\gamma_{v}, t v \in N_{K}$ is called a focal vector, and its multiplicity is by definition the dimension of $\operatorname{ker}\left(\exp _{*}\right)$. A point $q \in K$ is called a focal point if it is focal along some minimizing geodesic $\gamma_{v}$. Clearly, if $K$ is a point this reduces to the classical definition of conjugate points. Analogously to this latter situation, the set of focal points is discrete (Morse lemma, [Sak96]) and a geodesic ceases to be length minimizing after the first focal point, which implies $\rho(v) \leq \lambda(v)$ for every $v \in U_{K}$. The regularity of $\rho$ and $\lambda$ has been investigated by J.I. Itoh and M. Tanaka [IT01a], and Y. Li and L. Nirenberg [LN05] (see also the recent reference $[\mathbf{C R}])$. In both papers, the authors prove that $\rho$ and $\lambda$ are Lipschitz functions on the pre-image of compact intervals, where Lipschitz continuity is with respect to any fixed metric on $U_{K}$. Furthermore, $\rho$ and $\lambda$ are continuous if $(0,+\infty]$ is endowed with the topology having $\{(a,+\infty]: a>0\}$ as neighbourhoods of $+\infty$ (for $\rho$, this result goes back to M. Morse). Hence, the sets $U_{\rho}=\rho^{-1}\left(\mathbb{R}^{+}\right)$and $U_{\lambda}=\lambda^{-1}\left(\mathbb{R}^{+}\right)$are open subsets of $U_{K}$ and

$$
e_{\rho}: v \in U_{\rho} \rightarrow \exp (\rho(v) v) \in M, \quad e_{\lambda}: v \in U_{\lambda} \rightarrow \exp (\lambda(v) v) \in M
$$

are Lipschitz continuous on the pre-image of compact sets. A vector $v \in U_{\rho}$ for which $\rho(v)=\lambda(v)$ is called a focal cut-vector, and $e_{\rho}(v)$ is called a focal cut-point. The set $e_{\rho}\left(U_{\rho}\right)$ is called the cut-locus of $K$, $\operatorname{cut}(K)$.

Theorem 1.1 ([Sak96], [GHL90]). Let $M, K, N_{K}, U_{K}, \rho$ be as above. Then, the following properties hold:

- (M. Morse) $M$ is compact if and only if $U_{\rho} \equiv U_{K}$ and $K$ is compact;

- $\exp$ is a diffeomorphism between the open sets $W=\left\{t v: v \in U_{K}, t \in\right.$ $(0, \rho(v))\}$ and $M \backslash(K \cup \operatorname{cut}(K))$, furthermore $M=\exp (\bar{W})$;

- every $q \in M \backslash(K \cup \operatorname{cut}(K))$ is joined to $K$ by a unique minimizing geodesic, and $d_{K}$ is smooth on $M \backslash(K \cup \operatorname{cut}(K))$.

- (W. Klingenberg) if $q \in \operatorname{cut}(K)$, then either there exist at least two distinct segments from $K$ to $q$, or $q$ is focal for $K$. The two possibilities do not reciprocally exclude;

- if $q \in \operatorname{cut}(K)$ is non-focal, then there exists only a finite number of segments joining $q$ to $K$.

The cut-locus of $K$ can be subdivided into the following subsets:

- the focal cut-locus $\operatorname{cut}_{f}(K)$, that is, the set of focal cut-points;

- the normal cut-locus $\operatorname{cut}_{n}(K)$, consisting of the non-focal cut-points joined to $K$ by exactly two distinct segments;

- the anormal cut-locus $\operatorname{cut}_{a}(K)$, consisting of non-focal cut-points joined to $K$ by at least three distinct segments.

Furthermore, we split the focal cut-locus according to the multiplicity of each focal cut-point. 
- the set of focal cut-points $q$ such that whenever $\rho(v) v$ is a focal vector, where $v \in e_{\rho}^{-1}(\{q\})$, the multiplicity of $\rho(v) v$ is 1 . We call it $\operatorname{cut}_{f 1}(K)$;

- the set of focal points $q$ such that there exists a unit vector $v \in e_{\rho}^{-1}(\{q\})$ such that $\rho(v) v$ has multiplicity at least 2 . We call it $\operatorname{cut}_{f 2}(K)$.

The structure of the non-focal part of the cut-locus has been dealt with in detail by V. Ozols [Ozo74], and by P. Hartman [Har64a] for the 2-dimensional case. Briefly, the normal cut-points are a smooth embedded $(m-1)$-submanifold without boundary and with at most countably many connected components. Furthermore, for every $q \in \operatorname{cut}_{n}(K)$ there exists a neighbourhood $V$ of $q$ such that

$$
\operatorname{cut}(K) \cap V \equiv \operatorname{cut}_{n}(K) \cap V,
$$

and $\operatorname{cut}_{n}(K)$ bisects the angle between the two segments from $K$ to $q$. On the preimage $e_{\rho}^{-1}\left(\operatorname{cut}_{n}(K)\right)$ the function $\rho$ is smooth, and $\mathrm{d}_{v} \rho=0$ at some $v$ if and only if the two segments from $K$ to $e_{\rho}(v)$ meet orthogonally to $\mathrm{cut}_{n}(K)$, that is, if they are part of a unique geodesic. According to the terminology introduced by K. Grove and K. Shiohama in [GS77], a normal cut-point $q$ such that $\mathrm{d} \rho=0$ on $e_{\rho}^{-1}(\{q\})$ is called a normal critical cut-point. We agree on denoting with $\operatorname{cut}_{n c}(K)$ the set of normal critical cut-points of $K$. We now turn to the anormal cut-locus. Around each anormal cut-point, the graph of $\operatorname{cut}(K)$ is a finite intersection of submanifolds with boundary, and at least two of them are transverse. Furthermore, $\operatorname{cut}_{n}(K)$ is dense in a neighbourhood of each anormal cut-point ([IT98], Lemma 2). Hence, $\operatorname{cut}_{a}(K)$ is locally a subset of a finite union of submanifolds whose dimensions do not exceed $(m-2)$. In particular, if $m=2$ anormal cut-points are isolated, as observed in [Har64a], Lemma 5.1. The above implies that the Hausdorff dimension $\operatorname{dim}_{\mathcal{H}}\left(\operatorname{cut}_{a}(K)\right)$ is at most $(m-2)$, see [IT98], Lemma 3. As for the focal part, by the Sard-Federer theorem ([Sar58] and [Fed69]) applied to exp : $N_{K} \rightarrow M$ the Hausdorff dimension of $\operatorname{cut}_{f 2}(K)$ is at most $(m-2)$. For the set $\operatorname{cut}_{f 1}(K)$ the situation is more subtle. Around each vector $v_{0} \in e_{\rho}^{-1}(\{q\}), q \in \operatorname{cut}_{f 1}(K)$, by the Malgrange preparation theorem the function $\lambda$ is smooth ([IT01b], Lemma 1). A clever argument ([IT98], Lemma 1) shows that the tangent space to the set $\left\{\lambda(v) v: v \in U_{\lambda}\right\}$ at $\lambda\left(v_{0}\right) v_{0}$ is a subset of $\operatorname{ker}\left(\exp _{*}\right)$, so that the map $e_{\lambda}$ is smooth and has rank $(m-2)$ in a neighbourhood of $v_{0}$. Hence, again by Sard-Federer theorem for $e_{\lambda}, \operatorname{dim}_{\mathcal{H}}\left(\operatorname{cut}_{f 1}(K)\right)=m-2$. To conclude,

$$
\operatorname{dim}_{\mathcal{H}}\left(\operatorname{cut}_{a}(K) \cup \operatorname{cut}_{f}(K)\right) \leq m-2,
$$

and the Hausdorff dimension of $\operatorname{cut}(K)$ is at most $(m-1)$. We mention that, with some further work, it can be proved that $\operatorname{dim}_{\mathcal{H}}(\operatorname{cut}(K))$ is always an integer around each cut-point, see [IT98]. If $m=2$, since $d_{K}$ is Lipschitz we also deduce that

$$
d_{K}\left(\operatorname{cut}_{a}(K) \cup \operatorname{cut}_{f}(K)\right) \text { has Lebesgue measure zero on } \mathbb{R}^{+} \text {if } m=2 \text {. }
$$

Indeed, we recall that the Hausdorff 1-measure coincides with Lebesgue measure on $\mathbb{R}$. Combining (1.1) and the fact that $\operatorname{cut}_{n}(K)$ is dense around each anormal cut-point, we deduce that

$$
\operatorname{dim}_{\mathcal{H}}(\operatorname{cut}(K))<m-1 \quad \text { if and only if } \quad \operatorname{cut}(K) \equiv \operatorname{cut}_{f}(K) .
$$

It is easy to construct non-compact manifolds $M$ with the property that, for some compact submanifold $K, \operatorname{cut}(K)$ is non-empty and has only focal points. For instance, if $m \geq 3$, consider a $j$-dimensional Cartan-Hadamard manifold $N$, $1 \leq j<m-1$, let $M=\mathbb{S}^{m-j} \times N$ and let $K=E \times\{p\}$, where $E \subset \mathbb{S}^{m-j}$ is an 
equator and $p \in N$. It is worth to observe that $\mathrm{F}$. Warner has given a sufficient condition for $\operatorname{cut}(o) \equiv \operatorname{cut}_{f}(o)$ to hold on a complete, simply connected $M$. More precisely, by [War67], Theorem 1.3 it is enough that, for every geodesic issuing from $o$, the first focal point (if any) along $\gamma$ has multiplicity at least 2 .

Next, we consider the intersection of the cut-locus with geodesic spheres.

Proposition 1.2 ([GP94], Lemma 1.1). The intersection cut $(K) \cap \partial B_{r}$ can be decomposed as $\operatorname{cut}_{n c}(K) \cup B$, where $\operatorname{dim}_{\mathcal{H}}(B) \leq m-2$.

Proof. Define $B$ to be the complementary of $\operatorname{cut}_{n c}(K)$ in $\partial B_{r}$. Then, $B$ is a subset of

$$
\operatorname{cut}_{f}(K) \cup \operatorname{cut}_{a}(K) \cup\left(\left(\operatorname{cut}_{n}(K) \backslash \operatorname{cut}_{n c}(K)\right) \cup \partial B_{r}\right) .
$$

Observe that $\partial B_{r}$ is included in $\exp \left(r U_{K}\right)$. Since $\operatorname{dim}_{\mathcal{H}}\left(\operatorname{cut}_{a}(K) \cup \operatorname{cut}_{f}(K)\right) \leq$ $m-2$, we are left to consider $A=\left(\operatorname{cut}_{n}(K) \backslash \operatorname{cut}_{n c}(K)\right) \cap \partial B_{r}$, that is, the set of normal, non critical cut-points $q$ in $\partial B_{r}$. For each such $q$, choose a sufficiently small neighbourhood $V$ of $q$ such that $\operatorname{cut}(K) \cap V$ contains only normal points, $\exp ^{-1}(V)=V_{1} \cup V_{2}$ and $V_{1} \cap V_{2}=\emptyset$. Let $\gamma_{1}, \gamma_{2}$ be the two segments from $K$ to $q$, where $\gamma_{i}=\exp \left(t v_{i}\right)$ and $\rho\left(v_{i}\right) v_{i} \in V_{i}$. By Gauss lemma, the tangent space to the smooth hypersurface $\exp \left(r U_{K} \cap V_{i}\right)$ at $q$ is orthogonal to $\gamma_{i}$. Since $q$ is non critical, the tangent space to $\operatorname{cut}_{n}(K)$ is transverse to the tangent space of $\exp \left(r U_{K} \cap V_{i}\right)$ for each $i \in\{1,2\}$. Thus, up to shrinking $V$, it follows by transversality that locally $A \cap V_{i}$ is a connected, regular $(m-2)$-dimensional submanifold. Since $M$ is second countable, we can cover $A$ with countably many such neighbourhoods $V$. Hence $\operatorname{dim}_{\mathcal{H}}(A)=m-2$. This proves the proposition.

REMARK 1.3. The set of normal critical values $d_{K}\left(\operatorname{cut}_{n c}(K)\right)$ has Lebesgue measure zero by Sard-Federer theorem. Indeed, $d_{K}\left(\operatorname{cut}_{n c}(K)\right)$ is the set of critical values of the smooth function $\rho$ on the open set (with countably many connected components) $e_{\rho}^{-1}\left(\operatorname{cut}_{n}(K)\right)$. We note in passing that, in their celebrated paper [GS77], K. Grove and K. Shiohama extended the definition of a critical point to cover the case of the distance function $d_{K}$, a definition that turned out to be extremely fruitful. Recently, the Morse-Sard theorem for the distance function, namely the assertion that the set of critical values of $d_{K}$ has Lebesgue measure zero, has been proved by Itoh and Tanaka [IT01b] for manifolds $M$ of dimension $m \leq 4$, and by L. Rifford [Rif04] for every $m$.

Combining with observation (1.2), we deduce the following Proposition for complete surfaces.

Proposition 1.4 ([Har64a], Proposition 6.1). Let $M$ be a connected, complete surface, and let $K$ be either a smooth, embedded, simple closed curve or a point. Then, with the exception of a closed set $Z$ of Lebesgue measure zero, $\partial B_{r}$ is a union of finitely many smooth, simple curves, each of them possibly having a finite number of corners.

PROOF. By Remark 1.3 and observation (1.2),

$$
d_{K}\left(\operatorname{cut}_{a}(K) \cup \operatorname{cut}_{f}(K) \cup \operatorname{cut}_{n c}(K)\right) \quad \text { has Lebesgue measure zero on } \mathbb{R}^{+} \text {. }
$$

It is not hard to see that $Z=\operatorname{cut}_{a}(K) \cup \operatorname{cut}_{n c}(K) \cup \operatorname{cut}_{f}(K)$ is closed. Let $r_{0} \in$ $\mathbb{R}^{+} \backslash Z$, and let $I$ be a small open neighbourhood of $r_{0}$ in $\mathbb{R}^{+} \backslash Z$. Then, $\operatorname{cut}(K) \cap$ 
$d_{K}^{-1}(I)$, if non-empty, has only normal, non-critical cut-points, so that for every $r \in I$ and $v_{1} \in e_{\rho}^{-1}\left(\operatorname{cut}(K) \cap \partial B_{r}\right)$ the graph manifold

$$
V_{\rho}=\left\{\rho(v) v: v \in U_{\rho}\right\} \subset N_{K}
$$

around $v_{1}$ is a smooth curve transverse to $r U_{K}$. Thus, $V_{\rho} \cap r U_{K}$, if non-empty, is an even number of isolated points $\left\{r v_{j}\right\}, j=\{1, \ldots, 2 h\}$, for some $h>0$. Applying the exponential map, the cut-vectors $r v_{j}$ meet together in pairs, and the resulting set

$$
\partial B_{r}=\exp \left(\left\{t v \in N_{K}: v \in U_{\rho}, t=\min \{r, \rho(v)\}\right\}\right)
$$

is a finite union of at most $h$ disjoint smooth simple curves, possibly with corners at the points of type $\exp \left(r v_{i}\right)=\exp \left(r v_{j}\right), i \neq j$. This concludes the proof.

As an immediate consequence, a Gauss-Bonnet inequality holds for almost every $r \in \mathbb{R}^{+}$.

Proposition 1.5. Let $M$ be a connected, complete surface and let $K$ be either a smooth simple closed curve or a point. Denote with $l(r)$ the Hausdorff 1-measure of the sphere $\partial B_{r}$ centered at $K$, with $\chi_{E}(r)$ the Euler characteristic of $B_{r}$ and with $k(r)$ the integral over $B_{r}$ of the Gaussian curvature of $M$. Then, for almost every $r>0$,

$$
l^{\prime}(r) \leq 2 \pi \chi_{E}(r)-k(r) .
$$

Now, we can start to describe more closely the regularity of the volume growth function. For every fixed $r$, consider the inclusion $i_{r}: r U_{K} \rightarrow N_{K}$ and define the smooth map $\exp _{r}=\exp \circ i_{r}: r U_{K} \rightarrow M$. We can endow $N_{K}$ with a metric $($, constructed in a way similar to that for the standard metric on $T M$ (see [Car92], p.78). Namely, for every $v \in N_{K}, \pi(v)=p \in K$ and $W, Z \in T_{v} N_{K}$ we choose curves $\alpha, \beta: I=[0,1] \rightarrow N_{K}$ such that

$$
\alpha(0)=\beta(0)=v, \quad \alpha^{\prime}(0)=W, \quad \beta^{\prime}(0)=Z
$$

and we define

$$
(W, Z)_{v}=\left\langle\pi_{*}(W), \pi_{*}(Z)\right\rangle_{p}+\left\langle\nabla_{t} \alpha, \nabla_{t} \beta\right\rangle_{p} .
$$

Then, (, ) is independent of the chosen curves, and the submanifolds $r U_{K}, r \in \mathbb{R}$ are orthogonal to the geodesic rays $t v, v \in U_{K}, t \in \mathbb{R}$ on common intersections. Indeed, $($, ) can be written as

$$
(,)=i_{r}^{*}(,)+\mathrm{d} r \otimes \mathrm{d} r .
$$

Having defined the $m$-dimensional (respectively, $(m-1)$-dimensional) Jacobian of $\exp \left(\right.$ resp. $\exp _{r}$ )

$$
J \exp =\left\|\bigwedge_{m} \mathrm{~d} \exp \right\|, \quad J \exp _{r}=\left\|\bigwedge_{m-1} \mathrm{~d} \exp _{r}\right\|,
$$

where the norm is taken with respect to $(),\left(\operatorname{resp}, i_{r}^{*}(),\right)$, the warped product structure (1.4) implies that $J \exp _{r}(v)=J \exp (r v)$ for every $t \in \mathbb{R}$ and $v \in U_{K}$. Let $\omega$ and $\omega_{r}$ be the volume form of $($,$) and the induced volume form on r U_{K}$. Then, up to the sign, $\omega=\omega_{r} \wedge \mathrm{d} r$. By the area formula ([EG92], Theorem 1 p.96; 
[Fed69], Theorem 3.2.3 and pp.280-282) applied to exp and to $\exp _{r}$ we deduce that, for every locally summable function $\chi$ on $N_{K}$ (resp. $r U_{K}$ ),

$$
\begin{aligned}
& \text { (i) } \int_{N_{K}} \chi(t v) J \exp (t v) \omega=\int_{M}\left[\sum_{t v \in \exp ^{-1}\{p\}} \chi(t v)\right] \mathrm{d} V(p) ; \\
& \text { (ii) } \int_{r U_{K}} \chi(r v) J \exp (r v) \omega_{r}=\int_{M}\left[\sum_{r v \in \exp ^{-1}\{p\}} \chi(r v)\right] \mathrm{d} \mathcal{H}^{m-1}(p),
\end{aligned}
$$

where $\mathrm{d} V$ is the Riemannian volume form of $M$ and $\mathrm{d} \mathcal{H}^{m-1}$ is the $(m-1)$ dimensional Hausdorff measure. We now consider a suitable $\chi$ on $\nu$. To be sure that the integrals are finite, we assume that $K$ is compact. For every vector $t v$, $v \in U_{K}, t \in \mathbb{R}_{0}^{+}$, we define $n(v)$ to be the number of distinct geodesic segments joining $K$ to $e_{\rho}(v)$. Let

$$
\chi_{t}(v)=\chi(t v)= \begin{cases}1 & \text { if } t<\rho(v) \\ n(v)^{-1} & \text { if } t=\rho(v) \\ 0 & \text { if } t>\rho(v) .\end{cases}
$$

Fix $r>0$. By taking the limit as $t \uparrow r$ and $t \downarrow r$ of $\chi(t v)$ we can define also the following functions:

$$
\chi_{r^{+}}(v)=\lim _{t \downarrow r} \chi(t v)=\left\{\begin{array}{ll}
1 & \text { if } r<\rho(v) ; \\
0 & \text { if } r \geq \rho(v) .
\end{array} \quad \chi_{r^{-}}(v)=\lim _{t \uparrow r} \begin{cases}1 & \text { if } r \leq \rho(v) ; \\
0 & \text { if } r>\rho(v) .\end{cases}\right.
$$

Applying (1.5), (ii) to $\chi_{r}$ we obtain

$$
\int_{r U_{K}} \chi(r v) J \exp (r v) \omega_{r}=\mathcal{H}^{m-1}\left(\partial B_{r}\right)=\operatorname{vol}\left(\partial B_{r}\right),
$$

while using (ii) first to $\chi_{t}$ and then to $\chi_{r^{-}}$, with the aid of Lebesgue convergence theorem we deduce

$$
\begin{aligned}
& \lim _{t \rightarrow r^{-}} \operatorname{vol}\left(\partial B_{t}\right)=\lim _{t \rightarrow r^{-}} \int_{t U_{K}} \chi(t v) J \exp (t v) \omega_{t}=\int_{r U_{K}} \chi\left(r^{-} v\right) J \exp (r v) \omega_{r} \\
& =\operatorname{vol}\left(\partial B_{r} \backslash \operatorname{cut}(K)\right)+\int_{\partial B_{r} \cap \operatorname{cut}(K)} \mathcal{H}^{0}\left(\exp ^{-1}\{p\}\right) \mathrm{d} \mathcal{H}^{m-1}(p) .
\end{aligned}
$$

This shows that the left limit of $v(r)$ exists for every $r>0$. Analogously,

$$
\lim _{t \rightarrow r^{+}} \operatorname{vol}\left(\partial B_{t}\right)=\operatorname{vol}\left(\partial B_{r} \backslash \operatorname{cut}(K)\right) .
$$

Setting $v(r)=\operatorname{vol}\left(\partial B_{r}\right)$ for convenience, from (1.8) and (1.9) we get

$$
v\left(r^{+}\right)-v\left(r^{-}\right)=-\int_{\partial B_{r} \cap \operatorname{cut}(K)} \mathcal{H}^{0}\left(\exp ^{-1}\{p\}\right) \mathrm{d} \mathcal{H}^{m-1}(p) .
$$

By Proposition 1.2, $\partial B_{r} \cap \operatorname{cut}(K)$ can be decomposed as the set of normal critical points in $\partial B_{r}$ plus a set of Hausdorff dimension at most $(m-2)$. Hence, the integral in (1.10) coincides with the integral over all the normal critical points in 
$\partial B_{r}$. Therefore,

$$
\begin{aligned}
v\left(r^{+}\right)-v\left(r^{-}\right) & =-\int_{\partial B_{r} \cap \operatorname{cut}_{n c}(K)} \mathcal{H}^{0}\left(\exp ^{-1}\{p\}\right) \mathrm{d} \mathcal{H}^{m-1}(p) \\
& =-2 \operatorname{vol}\left(\partial B_{r} \cap \operatorname{cut}_{n c}(K)\right)
\end{aligned}
$$

It follows that $v(r)$ jumps downward every time $\partial B_{r}$ meets nontrivial portions of the normal critical cut-locus. The following proposition collects the basic properties of the volume function that will be needed in the next chapters

Proposition 1.6. Let $M$ be a connected, complete, non-compact Riemannian manifold, and let $K \subset M$ be a compact embedded submanifold of dimension $k$. Then, $v(r)=\operatorname{vol}\left(\partial B_{r}\right)$ is smooth in a neighbourhood of $r=0$. Furthermore,

$$
\begin{aligned}
& \text { (i) } \quad \text { if } k=m-1, \text { then } \quad v(0)=\operatorname{vol}(K)>0, v^{\prime}(0)=0 \\
& \text { (ii) } \quad \text { if } k \leq m-2, \text { then } \quad v(0)=0, v^{\prime}(r)>0 \text { for positive } r \text { around } 0 ; \\
& \text { (iii) } \quad v(r) \in L_{\mathrm{loc}}^{\infty}([0,+\infty)), \quad v(r)>0 \text { for } r>0, \quad \frac{1}{v(r)} \in L_{\mathrm{loc}}^{\infty}((0,+\infty)) \text {; } \\
& \text { (iv) } \quad v(r)=\frac{v\left(r^{+}\right)+v\left(r^{-}\right)}{2}
\end{aligned}
$$

Proof. Using the normal exponential map near $K$ and a covering argument, by the compactness of $K$ there exists $\varepsilon>0$ such that $\exp : B_{\varepsilon}(0) \rightarrow B_{\varepsilon}$ is a diffeomorphism, where 0 means the set of zero vectors. Thus, for $r<\varepsilon, \partial B_{r}$ is contained in the domain of normal geodesic coordinates, hence $\chi(t v)=1$ for every $v \in U_{K}, t \in[0, \varepsilon)$ and $v(r)$ is smooth by formula (1.7). By the divergence theorem and coarea formula,

$$
v^{\prime}(r)=\frac{\mathrm{d}}{\mathrm{d} r}\left(\operatorname{vol}\left(\partial B_{r}\right)-\operatorname{vol}\left(\partial B_{\delta}\right)\right)=\frac{\mathrm{d}}{\mathrm{d} r}\left(\int_{B_{r} \backslash B_{\delta}} \Delta r\right)=\int_{\partial B_{r}} \Delta r .
$$

As for $(i)$, suppose first that $K$ is orientable and that $\exp : B_{\varepsilon}(0) \approx K \times(-\varepsilon, \varepsilon) \rightarrow$ $B_{\varepsilon}$ is a double collar (this is always the case if, for instance, $M$ is orientable). Denote with $\nu_{+}$and $\nu_{-}$the two orientations of $M$. Then, for $r<\varepsilon, \partial B_{r}$ has two connected components $\Sigma_{+, r}$ and $\Sigma_{-, r}$, where the signs,+- are chosen coherently with the orientations. Setting, for each $p \in K, p_{r}^{+}=\exp (p, r) \in \Sigma_{+, r}$ and $p_{r}^{-}=$ $\exp (p,-r) \in \Sigma_{-, r}$, by Gauss lemma $\Delta r\left(p_{r}^{+}\right)$(resp. $\left.\Delta r\left(p_{r}^{-}\right)\right)$is the mean curvature of $\Sigma_{+, r}\left(\right.$ resp. $\left.\Sigma_{-, r}\right)$ at $p_{r}^{+}$(resp. $p_{r}^{-}$). Letting $r \rightarrow 0^{+}, \Delta r\left(p_{r}^{ \pm}\right) \rightarrow \pm H$, where $H$ is the mean curvature of $K$ with respect to $\nu_{+}$. Thus, letting $r \rightarrow 0^{+}$in (1.13)

$$
v^{\prime}(0)=\lim _{r \rightarrow 0^{+}} \int_{\Sigma_{+, r} \cup \Sigma_{-, r}} \Delta r=\int_{K}(H-H)=0 .
$$

The other possibilities for $K$ (that is, $K$ is orientable but without any double collar, or $K$ is non-orientable) can be dealt with in a similar manner.

To show $(i i)$, it is enough to extend the computations in normal coordinates performed in [Pet97], Section 5.6 for $K=\{o\}$ to cover the case of general $K$. The simple method of the author allows a clean extension. Let $\left\{x^{i}, x^{\alpha}\right\}$ be coordinates on $M$ such that $\left\{x^{i}\right\}$ are coordinates on $K$ and $\left\{x^{\alpha}\right\}$ are the standard coordinates on the fibers of $N_{K}$ composed with the exponential map. Writing the metric as

$$
\langle,\rangle=g_{i j} \mathrm{~d} x^{i} \otimes \mathrm{d} x^{j}+g_{i \alpha} \mathrm{d} x^{i} \otimes \mathrm{d} x^{\alpha}+g_{\beta j} \mathrm{~d} x^{\beta} \otimes \mathrm{d} x^{j}+g_{\alpha \beta} \mathrm{d} x^{\alpha} \otimes \mathrm{d} x^{\beta},
$$


the Hessian of $r$ has the following behaviour as $r \rightarrow 0^{+}$

$$
\text { Hess } r=\frac{1}{r}\left(g_{\alpha \beta} \mathrm{d} x^{\alpha} \otimes \mathrm{d} x^{\beta}-\mathrm{d} r \otimes \mathrm{d} r\right)+O(1) \quad \text { as } r \rightarrow 0^{+} .
$$

(indeed, if $K=\{o\}$ the remaining is $o(1)$, but it is unessential). Tracing, we get

$$
\Delta r=\frac{m-1-k}{r}+O(1) \quad \text { as } r \rightarrow 0^{+} .
$$

Since $k \leq m-2$, then clearly $v(0)=\operatorname{vol}(K)=0$ and, if $r$ is sufficiently small, by (1.15) $\Delta r>0$ on $\partial B_{r}$, which gives $v^{\prime}(r)>0$. From (1.11)

$$
\begin{aligned}
v(r) & =\operatorname{vol}\left(\partial B_{r} \backslash \operatorname{cut}(K)\right)+\operatorname{vol}\left(\partial B_{r} \cap \operatorname{cut}(K)\right) \\
& =\operatorname{vol}\left(\partial B_{r} \backslash \operatorname{cut}(K)\right)+\operatorname{vol}\left(\partial B_{r} \cap \operatorname{cut}_{n c}(K)\right) \\
& =v\left(r^{+}\right)+\frac{v\left(r^{-}\right)-v\left(r^{+}\right)}{2}=\frac{v\left(r^{+}\right)+v\left(r^{-}\right)}{2},
\end{aligned}
$$

which proves assertion (iv). As for (iii), $v \in L_{\mathrm{loc}}^{\infty}([0,+\infty))$ follows from (1.7), since $\chi$ is bounded and the other terms vary continuously with $r$. Next, observe that if we prove that $1 / v \in L_{\mathrm{loc}}^{\infty}((0,+\infty))$, then $v(r)>0$ on $(0,+\infty)$. Indeed, assume $v\left(r_{0}\right)=0$ for some $r_{0} \in(0,+\infty)$. Then necessarily $v\left(r_{0}^{+}\right)=0, v\left(r_{0}^{-}\right)=$ $2 v\left(r_{0}\right)-v\left(r_{0}^{+}\right)=0$ and $1 / v$ is unbounded in a neighborhood of $r_{0}$. It remains to prove that $1 / v \in L_{\mathrm{loc}}^{\infty}((0,+\infty))$, that is, $v(r)$ is bounded away from zero on every compact set $C$ disjoint from $r=0$. Assume by contradiction that there exists $\left\{r_{k}\right\} \subset C$ such that $v\left(r_{k}\right) \rightarrow 0$. By compactness, and by $(i v)$, there exists $\widetilde{r} \in C$ such that $r_{k} \leftrightarrow \widetilde{r}$ and $v\left(\widetilde{r}^{+}\right)=0$. We are going to show that

$$
\partial B_{\widetilde{r}} \subseteq \operatorname{cut}(K) .
$$

Indeed, let (1.16) be false, and let $q \in \partial B_{\widetilde{r}} \backslash \operatorname{cut}(K)$. Then, we can choose a unique $v \in U_{K}$ such that $q=e_{\rho}(v)$, a neighbourhood $U$ of $v$ in $U_{K}$ such that $\tilde{r}<\rho(w)$ for every $w \in U$, and a neighbourhood $V$ with compact closure of the form

$$
V=\left\{r w: r \in\left(\widetilde{r}-\varepsilon_{0}, \widetilde{r}+\varepsilon_{0}\right), w \in U\right\},
$$

where $\varepsilon_{0}>0$ is sufficiently small. On $V, J \exp$ is strictly positive, thus there exists $C>0$ independent of $\varepsilon_{0}$ such that, for every $v \in U$ and $\varepsilon \leq \varepsilon_{0}$,

$$
J \exp ((\widetilde{r}+\varepsilon) v) \geq C J \exp (\widetilde{r} v), \quad \omega_{\widetilde{r}+\varepsilon} \geq C \omega_{\widetilde{r}}
$$

It follows that, by $(1.7)$, for every $\varepsilon \in\left(0, \varepsilon_{0}\right)$

$$
v(\widetilde{r}+\varepsilon)=\int_{(\widetilde{r}+\varepsilon) U_{K}} \chi((\widetilde{r}+\varepsilon) v) J \exp ((\widetilde{r}+\varepsilon) v) \omega_{\widetilde{r}+\varepsilon} \geq C \int_{r U_{K} \cap V} J \exp \omega_{\widetilde{r}}
$$

This contradicts $v\left(\widetilde{r}^{+}\right)=0$ and proves (1.16). By (1.16) we deduce that, for every geodesic ray $\gamma_{v}$ starting from $K$, there exists $t_{v} \leq r$ such that $\gamma_{v}\left(t_{v}\right) \in \operatorname{cut}(K)$, that is, $\rho(v)<+\infty$. Therefore, $U_{\rho} \equiv U_{K}$ and, since $K$ is compact, $M$ is compact by Theorem 1.1, against our assumptions.

Corollary 1.7. In the assumptions of Proposition 1.6, $v(r)$ has at most a countable number of discontinuities.

Proof. Define

$$
Q(r)=e_{\rho}^{-1}\left(\partial B_{r} \cap \operatorname{cut}_{n c}(K)\right) .
$$


By (1.11), $2 \operatorname{vol}\left(e_{\rho}(Q(r))\right)$ is the downward jump of $v(r)$. The sets $Q(r)$ are pairwise disjoint in $U_{K}$. Write $|\cdot|$ for the measure induced on $U_{K}$ by $i_{1}^{*}\left(\right.$, ). Since $U_{K}$ is compact, $\left|U_{K}\right|<+\infty$ so that each $A_{i}=\{r \geq 0:|Q(r)|>1 / i\}, i \in \mathbb{N}$, has finitely many elements, whence $A=\bigcup_{i=1}^{\infty} A_{i}$ is at most countable. To prove the sought it is enough to show that, if $\operatorname{vol}\left(e_{\rho}(Q(r))\right)>0$, then $|Q(r)|>0$. Let $r$ be such that $\operatorname{vol}\left(e_{\rho}(Q(r))\right)>0$. By (1.5) and Proposition 1.2

$$
\operatorname{vol}\left(e_{\rho}(Q(r))\right)=\int_{r U_{K}} \psi(r v) J \exp (r v) \omega_{r}
$$

where $\psi(r v)=\frac{1}{2}$ if $v \in Q(r), 0$ otherwise. Hence,

$$
0<\operatorname{vol}\left(e_{\rho}(Q(r))\right)=\frac{1}{2} \int_{Q(r)} J \exp (r v) \omega_{r} \leq C_{1} \int_{Q(r)} J \exp (v) \omega_{1} \leq C_{2}|Q(r)|,
$$

for some $C_{1}=C_{1}(r)>0, C_{2}=C_{2}(r)>0$, as desired.

It can be shown that, if $M$ and $K$ are real analytic (anyway, the case $K=\{o\}$ is allowed), $v(r)$ is continuous on $\mathbb{R}^{+}$. The result has been proved by F. Fiala [Fia41] when $M$ is an analytic closed curve on an analytic surface $M$, and by R. Grimaldi and P. Pansu for general $M$ and $K=\{o\}$. The argument in [GP94], Theorem 2 is as follows: if by contradiction $Z=\partial B_{r} \cap \operatorname{cut}_{n c}(K)$ has positive Hausdorff measure, since $e_{\rho}$ is locally Lipschitz $e_{\rho}^{-1}(Z)$ has positive Hausdorff measure. Moreover, from the characterization

$$
e_{\rho}^{-1}(Z)=\left\{v \in U_{K}: \exp (2 r v) \in K\right\},
$$

$e_{\rho}^{-1}(Z)$ is an analytic subset of $U_{K}$. Hence, $e_{\rho}^{-1}(Z) \equiv U_{K}$. Consequently, $M \equiv$ $B_{r}(K)$ is compact, contradicting our assumptions.

We conclude this section by recalling an integral inequality for Riemann surfaces that extends the Gauss-Bonnet theorem. This has been addressed by [Har64a] and [ST93]. To deal with the regularity of $l(r)=\operatorname{vol}\left(\partial B_{r}\right)$ when $M$ is a complete Riemann surface, the authors defined the jump function ([Har64a], equation (6.10))

$$
J(r)=\sum_{0 \leq t \leq r} \int_{Q(t)} J \exp (t v) \omega_{t}
$$

where $Q(t)$ is as in (1.17) and the sum contains at most countably many elements by Corollary 1.7. Furthermore, they defined as $L(r)$ ([Har64a], equation (6.8)) what is in our notations $l\left(r^{-}\right)$. Then, they proved that $L(r)+J(r)$ is absolutely continuous on $\mathbb{R}^{+}$. By (1.17), (1.18) and Proposition 1.6 we deduce that

$$
l(r)=L(r)+\operatorname{vol}\left(\partial B_{r} \cap \operatorname{cut}_{n c}(K)\right)=L(r)+\frac{1}{2} \int_{Q(r)} J \exp (r v) \omega_{r}
$$

Hence, setting

$$
j(r)=\sum_{0 \leq t<r} \int_{Q(t)} J \exp (t v) \omega_{t}+\frac{1}{2} \int_{Q(r)} J \exp (r v) \omega_{r},
$$

$j(r)$ shares the same properties as $J(r)$ and $L(r)+J(r)=l(r)+j(r)$. With the aid of Proposition 1.5, Theorems 6.2 and Corollary 6.1 of [Har64a], together with Theorems 2.2 and 3.2 of [ST93] can be restated as follows. 
Proposition 1.8. Let $M$ be a connected, complete Riemann surface, and let $K$ be either a smooth, simple closed curve or a point. Set $l(r)=\operatorname{vol}\left(\partial B_{r}\right)$, and define $j$ as in (1.20). Then, the function

$$
l(r)+j(r)
$$

is absolutely continuous on $\mathbb{R}^{+}$. Furthermore, for every $0 \leq R_{1}<R_{2}$

$$
l\left(R_{2}\right)-l\left(R_{1}\right) \leq \int_{R_{1}}^{R_{2}} l^{\prime}(s) \mathrm{d} s \leq 2 \pi \int_{R_{1}}^{R_{2}} \chi_{E}(s) \mathrm{d} s-\int_{R_{1}}^{R_{2}} k(s) \mathrm{d} s .
$$

\subsection{Model manifolds and basic comparisons}

Let $(M,\langle\rangle$,$) denote a connected, complete Riemannian manifold of dimension$ $m \geq 2$, with volume element $\mathrm{d} V$. For every $x \in M$, let $r(x)$ be the distance function from a reference origin $o \in M$. As we observed in the previous section, $r(x)$ is Lipschitz on $M$ and smooth on $D_{o}=M \backslash(\{o\} \cup \operatorname{cut}(o))$. We recall that $o$ is called a pole if $\operatorname{cut}(o)=\emptyset$. Comparison results for the Hessian and the Laplacian of $r$ may be considered a first instance where an extensive use of ODE theory comes into play. The material covered by this section is mostly contained in Section 2 of [PRS08], which is itself motivated by the analytic approach of P. Petersen, [Pet97]. The reasoning relies on some comparisons theorems for Riccati type equations that follow from Sturm type arguments, which we briefly recall for the convenience of the reader.

Theorem 1.9 (Sturm arguments, [Swa68]). Let $G \in L_{\text {loc }}^{\infty}(\mathbb{R})$.

(1) Let $g_{1}, g_{2}$ be solutions of

$$
\left\{\begin{array}{l}
g_{1}^{\prime \prime}-G g_{1} \leq 0 \\
g_{1}(0)=0,
\end{array}, \quad\left\{\begin{array}{l}
g_{2}^{\prime \prime}-G g_{2} \geq 0 \\
g_{2}(0)=0,
\end{array} \quad \text { and } \quad 0<g_{1}^{\prime}(0) \leq g_{2}^{\prime}(0)\right. \text {. }\right.
$$

Let $I_{j}=\left(0, S_{j}\right), j \in\{1,2\}$, be the maximal interval where $g_{j}$ is positive. Then, $S_{1} \leq S_{2}, g_{1}^{\prime} / g_{1} \leq g_{2}^{\prime} / g_{2}$ and $g_{1} \leq g_{2}$ on $I_{1}$. If $g_{1}(S)=g_{2}(S)$ for some $S \in I_{1}$, then $g_{1} \equiv g_{2}$ on $[0, S)$.

(2) Let $g_{1}, g_{2}$ satisfy $g_{1}^{\prime \prime}-G g_{1} \leq 0, g_{2}^{\prime \prime}-G g_{2} \geq 0$ on $[a, b] \subset \mathbb{R}$. If $g_{2}(a)=$ $g_{2}(b)=0$ and $g_{2}(s) \neq 0$ for each $s \in(a, b)$, then either $g_{1}$ has a zero in $(a, b)$ or $g_{1}=k g_{2}$ on $[a, b]$, for some $k \in \mathbb{R}$.

Proof. (1) Let $I=I_{1} \cap I_{2}$. On it, we define $F=g_{2} g_{1}^{\prime}-g_{1} g_{2}^{\prime}$. Then, $F(0)=0$ and $F^{\prime} \leq 0$ on $I$, therefore $F \leq 0$ on $I$. It follows that, on $I,\left(g_{1} / g_{2}\right)^{\prime} \leq 0$, hence $g_{1}^{\prime} / g_{1} \leq g_{2}^{\prime} / g_{2}$. Since, by De L'Hopital theorem, $\left(g_{1} / g_{2}\right)\left(0^{+}\right) \leq 1$, we deduce that $g_{1} \leq g_{2}$ on $I$, and thus $S_{1} \leq S_{2}$, that is, $I=I_{1}$, as claimed. The equality case follows easily from the above reasoning. To prove (2), suppose that $g_{1}$ has no zeroes in $(a, b)$. Without loss of generality, we can assume that $g_{1}$ and $g_{2}$ are positive on $(a, b)$. Having defined $F$ as in (1) we obtain $F^{\prime} \leq 0$. Integrating on $[a, b]$ and using $g_{1} \geq 0, g_{2}(a)=g_{2}(b)=0, g_{2}^{\prime}(a) \geq 0$ and $g_{2}^{\prime}(b) \leq 0$ we deduce that necessarily $F^{\prime} \equiv 0$, hence $F$ is constant. Since $F(a) \leq 0$ and $F(b) \geq 0$ we deduce that $F \equiv 0$, so that $g_{1} / g_{2}$ is constant on $[a, b]$.

Corollary 1.10. Let $G \in L_{\mathrm{loc}}^{\infty}(\mathbb{R})$, and let $g_{1}, g_{2}$ be two distinct solutions of $g^{\prime \prime}-G g=0$. Then, the zeroes of $g_{1}$ interlace with those of $g_{2}$.

Proof. It follows immediately from Sturm argument (2) interchanging the role of $g_{1}$ and $g_{2}$. 
REMARK 1.11. As a straightforward consequence of the above Corollary, each function $g$ satisfying $g^{\prime \prime}-G g=0$ on $\mathbb{R}$ has the same number of zeroes, possibly infinite. Thus the ODE $g^{\prime \prime}-G g=0$ is oscillatory if some (hence any) solution $g$ has infinitely many zeroes, and nonoscillatory if some (any) solution has only finitely many zeroes. We point out that the number of zeroes of each solution is related to the spectral theory of $-\mathrm{d}^{2} / \mathrm{d} s^{2}+G$ on the real line. The interested reader can consult [Wei87] for further study.

Next, we prove two variants of the comparison theorem for Riccati equations that follows from Sturm type arguments.

Proposition 1.12 (Riccati comparison). Let $I=\left[s_{0}, S\right)$ for some $-\infty<s_{0}<$ $S \leq+\infty$, and let $G \in C^{0}(I), \alpha>0$. Let $\phi_{i} \in A C(I), i=1,2$ be positive solutions respectively of the Riccati differential inequalities

$$
\phi_{1}^{\prime}+\frac{\phi_{1}^{2}}{\alpha} \leq \alpha G, \quad \phi_{2}^{\prime}+\frac{\phi_{2}^{2}}{\alpha} \geq \alpha G
$$

and suppose that $\phi_{1}\left(s_{0}\right) \leq \phi_{2}\left(s_{0}\right)$. Then, $\phi_{1} \leq \phi_{2}$ on $I$.

Proof. The functions $g_{i}$ defined by

$$
g_{i}(s)=\exp \left(\int_{s_{0}}^{s} \frac{\phi_{i}(\tau)}{\alpha} \mathrm{d} \tau\right)
$$

satisfy $g_{1}\left(s_{0}\right)=g_{2}\left(s_{0}\right), g_{1}^{\prime}\left(s_{0}\right) \leq g_{2}^{\prime}\left(s_{0}\right)$ and

$$
g_{1}^{\prime \prime}-G g_{1} \leq 0, \quad g_{2}^{\prime \prime}-G g_{2} \geq 0 .
$$

The desired conclusion follows by applying Sturm argument.

Proposition 1.13. Let $G \in C^{0}\left(\mathbb{R}_{0}^{+}\right)$and let $\phi_{i} \in A C((0, S)), i=1,2$, be positive solutions respectively of the Riccati differential inequalities

$$
\phi_{1}^{\prime}+\frac{\phi_{1}^{2}}{\alpha} \leq \alpha G, \quad \phi_{2}^{\prime}+\frac{\phi_{2}^{2}}{\alpha} \geq \alpha G
$$

a.e. on $(0, S)$, for some $\alpha>0$, satisfying the asymptotic relation

$$
\phi_{i}(s)=\frac{\beta_{i}}{s}+O(1), \quad \text { as } s \rightarrow 0^{+},
$$

for some $0<\beta_{1} \leq \beta_{2}$. Assume that $\beta_{1}+\beta_{2}-\alpha \geq 0$. Then $\phi_{1} \leq \phi_{2}$ on $(0, S)$.

Proof. The idea is the same as above. Since $\widetilde{\phi}_{i}=\alpha^{-1} \phi_{i}$ satisfies the assumptions with $\alpha=1$ and $\beta_{i}$ replaced by $\beta_{i} / \alpha$, we may assume $\alpha=1$. Observing that $\phi_{i}(s)-\beta_{i} / s$ is integrable in a neighbourhood of zero, we set

$$
g_{i}(s)=s^{\beta_{i}} \exp \left\{\int_{0}^{s}\left(\phi_{i}(\tau)-\frac{\beta_{i}}{\tau}\right) d \tau\right\} .
$$

Then $g_{i}(0)=0$,

$$
g_{i}^{\prime}=\phi_{i} g_{i} \in A C((0, S)) \quad \text { and } \quad g_{1}^{\prime \prime}-G g_{1} \leq 0, \quad g_{2}^{\prime \prime}-G g_{2} \geq 0 .
$$

From (1.22), $g_{i}^{\prime} \sim \beta_{i} s^{\beta_{i}-1}$ as $s \rightarrow 0^{+}$. Now, we apply Sturm argument: from (1.24) we deduce $\left(g_{1} g_{2}^{\prime}-g_{2} g_{1}^{\prime}\right)^{\prime} \geq 0$. From

$$
g_{1}(s) g_{2}^{\prime}(s) \sim \beta_{2} s^{\beta_{1}+\beta_{2}-1}, \quad g_{2}(s) g_{1}^{\prime}(s) \sim \beta_{1} s^{\beta_{1}+\beta_{2}-1} \quad \text { as } s \rightarrow 0^{+},
$$


and the assumptions $\beta_{1}+\beta_{2}-\alpha \geq 0$ and $0<\beta_{1} \leq \beta_{2}$, we get $\lim _{s \rightarrow 0^{+}}\left(g_{1} g_{2}^{\prime}-g_{2} g_{1}^{\prime}\right) \geq$ 0 , hence $g_{1} g_{2}^{\prime}-g_{2} g_{1}^{\prime} \geq 0$ on $(0, S)$, that is,

$$
\phi_{2}=\frac{g_{2}^{\prime}}{g_{2}} \geq \frac{g_{1}^{\prime}}{g_{1}}=\phi_{1},
$$

and this concludes the proof.

The comparison theory for Riccati equations can be implemented in the matrixvalued setting. Let $E$ be a finite dimensional vector space endowed with an inner product $\langle$,$\rangle and induced norm \|\cdot\|$, and let $S(E)$ be the space of self-adjoint linear endomorphisms of $E$. We say that $A \in S(E)$ satisfies $A \geq 0$ if $A$ is positive semidefinite. Analogously, we say that $A \leq B$ if $B-A$ is positive semidefinite. We denote with $I \in S(E)$ the identity transformation. The following comparison result is due to J.H. Eschenburg and E. Heintze [EH90].

Theorem 1.14 (Matrix Riccati comparison, [EH90]). Let $R_{i}: \mathbb{R}_{0}^{+} \rightarrow S(E)$, $i=1,2$ be smooth curves, and assume that $R_{1} \leq R_{2}$. For each $i$, let $B_{i}:\left(0, s_{i}\right) \rightarrow$ $S(E)$ be a maximally defined solution of the matrix Riccati equation

$$
B_{i}^{\prime}+B_{i}^{2}=R_{i} .
$$

Suppose that $U=B_{2}-B_{1}$ can be continuously extended at $s=0$ and $U\left(0^{+}\right) \geq 0$. Then,

$$
s_{1} \leq s_{2} \quad \text { and } \quad B_{1} \leq B_{2} \quad \text { on }\left(0, s_{1}\right) .
$$

Furthermore, $d(s)=\operatorname{dim} \operatorname{ker} U(s)$ is non-increasing on $\left(0, s_{1}\right)$. In particular, if $B_{1}(\widetilde{s})=B_{2}(\widetilde{s})$, then $B_{1} \equiv B_{2}$ on $(0, \widetilde{s})$.

Proof. Set $s_{0}=\min \left\{s_{1}, s_{2}\right\}$ and observe that, on $\left(0, s_{0}\right), U=B_{2}-B_{1}$ satisfies

$$
U^{\prime}=U X+X U+S, \quad \text { where } \quad\left\{\begin{array}{l}
S=R_{2}-R_{1} \geq 0 \\
X=-\frac{1}{2}\left(B_{2}+B_{1}\right)
\end{array}\right.
$$

We claim that $X$ is bounded from above near $s=0$. Indeed, by the Riccati equation $B_{i}^{\prime} \leq R_{i}$, hence for every unit vector $x \in E$ the function $\eta_{i}(s)=\left\langle B_{i}(s) x, x\right\rangle$ satisfies $\eta_{i}^{\prime} \leq\left\langle R_{i}(s) x, x\right\rangle \leq\left\|R_{i}(s)\right\| \leq C$, where the last inequality follows since $R_{i}$ is bounded on $\left[0, s_{0}\right]$. Integrating on some $[s, \widetilde{s}] \subset\left(0, s_{0}\right)$,

$$
\eta_{i}(s) \geq-C(\widetilde{s}-s)+\eta_{i}(\widetilde{s}) \geq-C \widetilde{s}-\left\|B_{i}(\widetilde{s})\right\|
$$

independently on $x$. Therefore, each $B_{i}$ is bounded from below as $s \rightarrow 0$, and thus there exists $a>0$ such that $X \leq a I$ near $s=0$, as claimed. The solution $U$ of (1.25) can be computed via the method of the variation of constants. First, fix $\widetilde{s} \in\left(0, s_{0}\right)$ and consider the solution $g$ of the Cauchy problem

$$
\left\{\begin{array}{l}
g^{\prime}=X g \\
g(\widetilde{s})=I
\end{array}\right.
$$

where $I \in S(E)$ is the identity. Then, $g$ is nonsingular on $\left(0, s_{0}\right)$ : indeed, its inverse is given by the function $\bar{g}$ satisfying $\bar{g}^{\prime}=-\bar{g} X, \bar{g}(\widetilde{s})=I$. The general solution $U$ of $(1.25)$ is thus

$$
U=g V g^{t}
$$

Where $V:\left(0, s_{0}\right) \rightarrow S(E)$ is the general solution of

$$
V^{\prime}=g^{-1} S\left(g^{-1}\right)^{t} .
$$


Since $S \geq 0$, we deduce $V^{\prime} \geq 0$. Hence, for every fixed $x \in E,\langle V(s) x, x\rangle$ : $\left(0, s_{0}\right) \rightarrow \mathbb{R}$ is non-decreasing. This shows that the pointwise limit $\langle V(0) x, x\rangle$ exists, possibly infinite. We claim that $\langle V(0) x, x\rangle$ is finite, hence $V(0)$ can be defined by polarization. Furthermore, we shall show that $V(0) \geq 0$. Towards this aim, from (1.26) and setting, for notational convenience, $h=\left(g^{-1}\right)^{t}$,

$$
\langle V x, x\rangle=\left\langle g^{-1} U\left(g^{t}\right)^{-1} x, x\right\rangle=\left\langle U\left(g^{-1}\right)^{t} x,\left(g^{-1}\right)^{t} x\right\rangle=\langle U h x, h x\rangle,
$$

so that

$$
|\langle V x, x\rangle| \leq\|U\| \cdot\|h x\|^{2} .
$$

Since, by assumption, $\|U\|$ is bounded as $s \rightarrow 0$, to prove that $|\langle V x, x\rangle|$ is bounded in a neighbourhood of zero we shall show that so is the function $f(s)=\|h(s) x\|^{2}$. Note that, by its very definition and the properties of $g, h^{\prime}=-X h$. Hence,

$$
f^{\prime}(s)=2\left\langle h^{\prime}(s) x, h(s) x\right\rangle=-2\langle X h(s) x, h(s) x\rangle \geq-2 a f .
$$

By Gronwall lemma, $f$ cannot diverge as $s \rightarrow 0^{+}$, as required. As a consequence, for every $s_{k} \rightarrow 0$ the set $\left\{y_{k}\right\}=\left\{h\left(s_{k}\right) x\right\} \subset E$ is bounded. By compactness, up to a subsequence $y_{k} \rightarrow y$, for some $y \in E$. Therefore, by (1.27)

$$
\langle v(0) x, x\rangle=\lim _{k}\left\langle V\left(s_{k}\right) x, x\right\rangle=\lim _{k}\left\langle U\left(s_{k}\right) y_{k}, y_{k}\right\rangle=\langle U(0) y, y\rangle \geq 0
$$

hence $V(0) \geq 0$. From $V^{\prime} \geq 0$, we deduce $V \geq 0$, thus by (1.26) $U \geq 0$, as desired. Since $V$ is non-negative and non-decreasing, so is $\operatorname{dim} \operatorname{ker} V(s)$. By (1.26), $\operatorname{dim} \operatorname{ker} V(s)=\operatorname{dim} \operatorname{ker} U(s)=d(s)$, and this concludes the proof.

We briefly recall the procedure that yields the classical Hessian, Laplacian and volume comparison theorems. In the notation of Section 1.2, let $p \in D_{o}$, and let $\gamma:[0, r(x)] \rightarrow M$ be the minimizing geodesic from $o$ to $p$, so that $r(\gamma(s))=s$ and $\nabla r \circ \gamma=\gamma^{\prime}$ for every $s$. Fix a local orthonormal coframe $\left\{e_{i}\right\}$ around $p$, with dual coframe $\left\{\theta^{i}\right\}, 1 \leq i \leq m$, so that the $(1,3)$-curvature tensor is given by

$$
R_{j k t}^{i} \theta^{k} \otimes \theta^{t} \otimes \theta_{j} \otimes e_{i}, \quad R_{j k t}^{i}=\left\langle R\left(e_{k}, e_{t}\right) e_{j}, e_{i}\right\rangle=-\left\langle R\left(e_{i}, e_{j}\right) e_{k}, e_{t}\right\rangle
$$

Then $\gamma^{\prime}=\nabla r=r_{i} e_{i}, \mathrm{~d} r=r_{i} \theta^{i}$ and differentiating $r_{i} r_{i}=1$ we obtain

$$
r_{i j} r_{i}=0 \quad \text { that is, } \operatorname{Hess} r(\nabla r, \cdot)=0 \text {. }
$$

A further covariant differentiation of (1.28) gives

$$
r_{i j k} r_{i}+r_{i j} r_{i k}=0
$$

By Schwarz symmetry of second derivatives of $r$ and the Ricci commutation rules

$$
u_{i j k}=u_{i k j}+u_{t} R_{i j k}^{t} \quad \forall u \in C^{3}(M)
$$

we get

$$
0=r_{i j k} r_{i}+r_{i j} r_{i k}=r_{j i k} r_{i}+r_{i j} r_{i k}=r_{j k i} r_{i}+r_{t} R_{j i k}^{t} r_{i}+r_{i j} r_{i k}
$$

Contracting the above relation with two parallel vector fields $X=X^{j} e_{j}, Y=Y^{j} e_{j}$ along $\gamma$ and perpendicular to $\nabla r$ we obtain

$$
0=r_{j k i} X^{j} Y^{k} r_{i}+X^{j} Y^{k} r_{t} r_{i} R_{j i k}^{t}+r_{i j} r_{i k} X^{j} Y^{k}
$$

Using Koszul notation and denoting with hess $r$ the $(1,1)$ version of Hess $r$, the above relation reads

$$
0=\langle\nabla \operatorname{hess} r(X ; \nabla r), Y\rangle+\langle\operatorname{hess}(r)(X) \text {, hess }(r)(Y)\rangle+\langle R(X, \nabla r) \nabla r, Y\rangle=0 \text {. }
$$


Since hess $r$ is self-adjoint, denoting with $R_{\gamma}$ the self-adjoint map

$$
X \mapsto R_{\gamma}(X)=R(X, \nabla r) \nabla r,
$$

and with a prime the covariant differentiation along $\gamma,(1.29)$ becomes

$$
0=\left\langle\left((\text { hess } r)^{\prime}+(\text { hess } r)^{2}+R_{\gamma}\right)(X), Y\right\rangle=0 \quad \forall X, Y \in \nabla r^{\perp} \text { parallel. }
$$

Note that, by (1.28) and the properties of the curvature tensor, both hess $r$ and $R_{\gamma}$ can be thought as endomorphisms of $\nabla r^{\perp}$. Furthermore, for every unit vector $X \in \nabla r^{\perp}$,

$$
\left\langle R_{\gamma}(X), X\right\rangle=K(X \wedge \nabla r)=K_{\text {rad }}(X),
$$

that is, the sectional curvature of $X \wedge \nabla r$. Since $X$ and $Y$ are arbitrary, we have

$$
(\text { hess } r)^{\prime}+(\text { hess } r)^{2}+R_{\gamma}=0
$$

as a section of $\operatorname{End}\left(\nabla r^{\perp}\right)$ along $\gamma$. By parallel translation, we can identify the fibres of the vector bundle $\nabla r^{\perp}$. Indeed, if we consider an orthonormal basis $\left\{E_{i}\right\} \subset \nabla r^{\perp}$ of parallel vector fields along $\gamma$, and we denote with $B=\left(r_{i j}\right), R_{\gamma}=\left(\left(R_{\gamma}\right)_{i j}\right)$ the representation of hess $r_{\mid \nabla r^{\perp}}$ and $R_{\gamma}$ in the basis $\left\{E_{i}\right\}$, (1.33) becomes the matrix Riccati equation

$$
B^{\prime}+B^{2}+R_{\gamma}=0
$$

Taking into account the asymptotic relation (1.14) for $K=\{o\}$

$$
\text { Hess } r=\frac{1}{s}(\langle,\rangle-\mathrm{d} r \otimes \mathrm{d} r)+o(1) \quad \text { as } s \rightarrow 0^{+},
$$

and $B$ satisfies

$$
\left\{\begin{array}{l}
B^{\prime}+B^{2}+R_{\gamma}=0 \quad \text { on } \quad(0, r(x)] \\
B(s)=s^{-1} I+o(1) \quad \text { as } s \rightarrow 0^{+} .
\end{array}\right.
$$

Now, assume either

$$
\text { (i) : } K_{\mathrm{rad}} \geq-G(r) \quad \text { or } \quad(i i): K_{\mathrm{rad}} \leq-G(r),
$$

for some $G \in C^{0}\left(\mathbb{R}_{0}^{+}\right)$. Henceforth, $(i)$ (resp. (ii)) means that the inequality

$$
K(\Pi)(x) \geq-G(r(x)),
$$

(resp, $\leq$ ) holds for every 2 -plane $\Pi$ containing $\nabla r$. Then, by (1.32), respectively

$$
\text { (i) : } R_{\gamma}(s) \geq-G(s) I, \quad(\text { ii }): R_{\gamma} \leq-G(s) I,
$$

and by (1.34) this yields the following matrix Riccati inequalities:

$$
\begin{aligned}
& \text { case }(i):\left\{\begin{array}{l}
B^{\prime}+B^{2} \leq G I, \\
B(s)=s^{-1} I+o(1) \quad \text { as } s \rightarrow 0^{+} ;
\end{array}\right. \\
& \text {case }(i i):\left\{\begin{array}{l}
B^{\prime}+B^{2} \geq G I, \\
B(s)=s^{-1} I+o(1) \quad \text { as } s \rightarrow 0^{+} ;
\end{array}\right.
\end{aligned}
$$


Now, consider a solution $g$ to

$$
\begin{aligned}
& \left\{\begin{array}{l}
g^{\prime \prime}-G g \geq 0 \\
g(0)=0, \quad g^{\prime}(0)=1
\end{array} \quad \text { for }(i),\right. \\
& \left\{\begin{array}{l}
g^{\prime \prime}-G g \leq 0 \\
g(0)=0, \quad g^{\prime}(0)=1
\end{array} \quad \text { for }(i i),\right.
\end{aligned}
$$

and assume that $g$ is positive on some maximal interval $I=\left(0, R_{0}\right)$. Setting $B_{g}=\left(g^{\prime} / g\right) I$, we have that

$$
\begin{aligned}
& \text { case }(i):\left\{\begin{array}{l}
B_{g}^{\prime}+B_{g}^{2} \geq G I, \\
B_{g}(s)=s^{-1} I+o(1) \quad \text { as } s \rightarrow 0^{+} ;
\end{array}\right. \\
& \text {case }(\text { ii }):\left\{\begin{array}{l}
B_{g}^{\prime}+B_{g}^{2} \leq G I, \\
B_{g}(s)=s^{-1} I+o(1) \text { as } s \rightarrow 0^{+} .
\end{array}\right.
\end{aligned}
$$

By the matrix Riccati Comparison 1.14, $B \leq B_{g}$ when $(i)$ holds, and $B \geq B_{g}$ under assumption (ii). Together with (1.28) and the definition of $B$ this yields the following

TheOREM 1.15 (Hessian comparison). Let $(M,\langle\rangle$,$) be a complete manifold of$ dimension $m$. Having fixed an origin $o$, let $r(x)$ be the distance function from $o$ and let $D_{o}=M \backslash(\{o\} \cup \operatorname{cut}(o))$ be the maximal domain of normal geodesis coordinated at o. Consider a function $G \in C^{0}\left(\mathbb{R}_{0}^{+}\right)$, let $g$ be the solution of the Cauchy problem

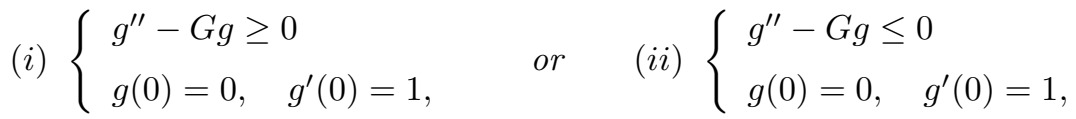

and let $\left(0, R_{0}\right)$ be the maximal interval in $\mathbb{R}^{+}$where $g>0$. Then,

(1) If the radial sectional curvature $K_{\mathrm{rad}}$ satisfy

$$
K_{\mathrm{rad}}(x) \geq-G(r(x)) \quad \text { on } B_{R_{0}}(o),
$$

then

$$
\text { Hess } r(x) \leq \frac{g^{\prime}(r(x))}{g(r(x))}(\langle,\rangle-\mathrm{d} r \otimes \mathrm{d} r) \quad \text { on } \quad D_{o} \cap B_{R_{0}}(o)
$$

in the sense of quadratic forms, where $g(r)$ solves $(i)$.

(2) If the radial sectional curvature $K_{\text {rad }}$ satisfy

$$
K_{\mathrm{rad}}(x) \leq-G(r(x)) \quad \text { on } B_{R_{0}}(o),
$$

then

$$
\operatorname{Hess} r(x) \geq \frac{g^{\prime}(r(x))}{g(r(x))}(\langle,\rangle-\mathrm{d} r \otimes \mathrm{d} r) \quad \text { on } \quad D_{o} \cap B_{R_{0}}(o)
$$

in the sense of quadratic forms, where $g(r)$ solves (ii).

The above theorem and the next ones are essentially comparisons with a model manifold in the sense of R.E. Greene and H. Wu, [GW79]. Since models will be repeatedly used in the rest of this work, we feel convenient to recall their definition and basic properties. 
Definition 1.16. A Riemannian manifold $\left(M_{g}, \mathrm{~d} s^{2}\right)$ is called a model if $M_{g}$ is diffeomorphic to $\mathbb{R}^{m}$, there exists a point $o \in M_{g}$ such that $\exp _{o}: T_{o} M_{g} \rightarrow M_{g}$ is a diffeomorphism, the metric $\mathrm{d} s^{2}$ is radially symmetric and writes, in global polar geodesic coordinates around $o$, as

$$
\mathrm{d} s^{2}=\mathrm{d} r^{2}+g(r)^{2} \mathrm{~d} \theta^{2}
$$

where with the symbol $\mathrm{d} \theta^{2}$ we mean the standard metric on the unit sphere $\mathbb{S}^{m-1}$, and $g \in C^{\infty}\left(\mathbb{R}_{0}^{+}\right), g>0$ on $\mathbb{R}^{+}$satisfies the following conditions at $r=0$ :

$$
g^{\prime}(0)=1, \quad g^{(2 k)}(0)=0 \quad \text { for every } k=0,1,2, \ldots
$$

Here, $g^{(j)}$ denotes the $j$-iterated derivative of $g$.

The conditions imposed on $g$ at $r=0$ are necessary and sufficient to ensure that $\mathrm{d} s^{2}$ can be smoothly extended in a neighbourhood of $o$. Typical examples of model manifolds are $\mathbb{R}^{m}$, for which $g(r)=r$, and the hyperbolic space $\mathbb{H}_{B}^{m}$ of sectional curvature $-B^{2}<0$, where $g(r)=B^{-1} \sinh (B r)$. A model manifold enjoys the following properties (see [Pet97], Section 1.4)

- The tangential sectional curvature at $x \in M_{g}, r(x)=r$, is $K(X \wedge Y)=$ $\left[1-\left(g(r)^{\prime}\right)^{2}\right] / g(r)^{2}$ for every orthogonal pair of unit vectors $X, Y \in \nabla r_{x}^{\perp}$.

- The radial sectional curvature at $x, r(x)=r$, is $K_{\mathrm{rad}}(X)=-g^{\prime \prime}(r) / g(r)$ for every unit vector $X \in \nabla r_{x}^{\perp}$. Consequently, the operator $R_{\gamma}$ in (1.30) is $-g^{\prime \prime} / g I$ and by $(1.33)$

$$
\text { Hess } r(x)=\frac{g^{\prime}(r)}{g(r)}\left(\mathrm{d} s^{2}-\mathrm{d} r \otimes \mathrm{d} r\right) \quad \text { on } \quad M_{g} \backslash\{o\} .
$$

- The Laplacian of $r$ at $x, r(x)=r$, is $\Delta r(x)=(m-1) g^{\prime}(r) / g(r)$; the volume of the geodesic spheres and balls centered at $o$ is, respectively, given by

$$
\operatorname{vol}\left(\partial B_{r}\right)=\omega_{m-1} g(r)^{m-1}, \quad \operatorname{vol}\left(B_{r}\right)=\omega_{m-1} \int_{0}^{r} g(s)^{m-1} \mathrm{~d} s,
$$

where $\omega_{m-1}$ is the volume of the unit sphere $\mathbb{S}^{m-1}$.

In what follows, we will often consider models with given radial sectional curvature $G(r)=-g^{\prime \prime}(r) / g(r) \in C^{\infty}\left(\mathbb{R}_{0}^{+}\right)$. Clearly, a model $\left(M_{g}, \mathrm{~d} s^{2}\right)$ is uniquely determined by $G$ once $g$ is a solution of

$$
\left\{\begin{array}{l}
g^{\prime \prime}-G g=0 \quad \text { on } \mathbb{R}^{+} \\
g(0)=0, \quad g^{\prime}(0)=1 .
\end{array}\right.
$$

Before considering the Laplacian and volume comparison theorems, we spend a few words on Jacobi tensors along geodesics, that can be easily constructed starting from the Riccati equation for hess $r$. For a more detailed treatment, see [EO80]. If $x, \gamma, R_{\gamma}, B$ are as in the proof of Theorem 1.15, consider the solution $W$ of the following problem:

$$
\left\{\begin{array}{l}
W^{\prime}=\left(B-s^{-1} I\right) W \quad \text { on }[0, r(x)] \\
W(0)=I .
\end{array}\right.
$$

Note that, from the asymptotic properties of $B$ in (1.35), $W$ is well defined and invertible on $[0, r(x)]$. The tensor field $J(s)=s W(s)$ is thus invertible on $(0, r(x)]$ 
and solves

$$
J^{\prime}=B J \quad \text { on }(0, r(x)] \text { and }\left\{\begin{array}{l}
J^{\prime \prime}+R_{\gamma} J=0 \quad \text { on }(0, r(x)] \\
J(0)=0, \quad J^{\prime}(0)=I .
\end{array}\right.
$$

By the linearity of (1.39), $J$ is smooth on $[0, r(x)]$ and can be smoothly extended on the whole $\mathbb{R}_{0}^{+} . J$ is called a Jacobi tensor along the geodesic $\gamma$. It is easy to see that $J$ is characterized by the property that, whenever $X \perp \gamma^{\prime}$ is a unit parallel vector field along $\gamma, J X \perp \gamma^{\prime}$ is a Jacobi field. Therefore, a point $y=\gamma\left(s_{1}\right)$ is conjugate to $o$ along $\gamma$ if and only if $J$ is not invertible at $s_{1}$. On the maximal interval where $J$ is invertible, say $\left(0, s_{1}\right)$, we can define a function $\widehat{B}$ by setting $\widehat{B}=J^{\prime} J^{-1}$. Then, by (1.39) $\widehat{B}$ extends $B$ and solves the Riccati equation (1.34). Moreover, if $s_{1}<+\infty$, $B$ cannot be defined past $s_{1}$. Indeed, let $X$ be a unit parallel vector field such that $J X\left(s_{1}\right)=0$. Then, since $J X \not \equiv 0,(J X)^{\prime}\left(s_{1}\right) \neq 0$. Therefore, by (1.39)

$$
\frac{\langle B J X, J X\rangle}{|J X|^{2}}=\frac{\left\langle J^{\prime} X, J X\right\rangle}{|J X|^{2}}=\frac{1}{2} \frac{\mathrm{d}}{\mathrm{d} s} \log |J X|^{2} \rightarrow-\infty \quad \text { as } s \rightarrow s_{1}^{-} .
$$

This means that the function hess $r \circ \gamma$ can be extended past the cut-point of $o$ along $\gamma$, if the cut-point is non-focal, and the maximal extension is defined on $\left(0, s_{1}\right)$, where $\gamma\left(s_{1}\right)$ is the first focal point of $o$ along $\gamma$. At $\gamma\left(s_{1}\right)$, however, hess $r \circ \gamma$ presents a singularity, and more precisely it is unbounded from below as $s \rightarrow s_{1}$.

The Laplacian comparison theorem from below is simply obtained by tracing the inequalities of the Hessian comparison Theorem 1.15, (2).

THEOREM 1.17 (Laplacian comparison from below). Let $(M,\langle\rangle$,$) be a complete$ manifold of dimension $m$ with a pole o. Consider a function $G \in C^{0}\left(\mathbb{R}_{0}^{+}\right)$, and let $g$ be the solution of the Cauchy problem

$$
\left\{\begin{array}{l}
g^{\prime \prime}-G g \leq 0 \\
g(0)=0, \quad g^{\prime}(0)=1
\end{array}\right.
$$

Suppose that $g>0$ on $\mathbb{R}^{+}$. Then, if

$$
K_{\mathrm{rad}}(x) \leq-G(r(x)) \quad \text { for every } \quad x \in M \backslash\{o\},
$$

the inequality

$$
\Delta r(x) \geq(m-1) \frac{g^{\prime}(r(x))}{g(r(x))}
$$

holds pointwise on $M \backslash\{o\}$ and weakly on $M$.

REMARK 1.18. The weak inequality is simple to show, since by (1.15) $\Delta r$ has an integrable singularity near $r=0$.

In particular, when $G(r)=B^{2}$ for some $B>0$ we can choose

$$
g(r)=B^{-1} \sinh (B r), \text { hence } \quad \Delta r(x) \geq(m-1) B \operatorname{coth}(B r(x)) \quad \text { on } M \backslash\{o\} .
$$

This last bound will be often applied in forecoming sections. However, a similar upper estimate for $\Delta r$ holds under the weaker assumption of a lower bound on the Ricci curvature, and even past the cut-locus, as the next theorem shows. 
TheOREM 1.19 (Laplacian comparison from above). In the notations of the previous theorem, assume that the radial Ricci curvature satisfy

$$
\operatorname{Ricc}(\nabla r, \nabla r)(x) \geq-(m-1) G(r(x)) \quad \text { on } D_{o},
$$

for some function $G \in C^{0}\left(\mathbb{R}_{0}^{+}\right)$, and let $g \in C^{2}\left(\mathbb{R}_{0}^{+}\right)$be a solution of

$$
\left\{\begin{array}{l}
g^{\prime \prime}-G g \geq 0 \\
g(0)=0, \quad g^{\prime}(0)=1 .
\end{array}\right.
$$

Let $\left(0, R_{0}\right)$ be the maximal interval where $g$ is positive. Then,

$$
D_{o} \subset B_{R_{0}}
$$

and the inequality

$$
\Delta r(x) \leq(m-1) \frac{g^{\prime}(r(x))}{g(r(x))}
$$

holds pointwise on $D_{o}$ and weakly on $M$.

Proof. Tracing (1.33) with respect to a parallel orthonormal frame $\left\{E_{j}\right\}$ for $\nabla r^{\perp}$ along $\gamma$, and using that

$$
\left\langle(\operatorname{hess} r)^{\prime}\left(E_{j}\right), E_{j}\right\rangle=\frac{\mathrm{d}}{\mathrm{d} s}\left\langle\operatorname{hess} r\left(E_{j}\right), E_{j}\right\rangle=\frac{\mathrm{d}}{\mathrm{d} s}\left(\operatorname{Hess} r\left(E_{j}, E_{j}\right)\right)
$$

we deduce

$$
(\Delta r)^{\prime}+|\operatorname{Hess} r|^{2}+\operatorname{Ricc}(\nabla r, \nabla r)=0 .
$$

From Newton inequality and (1.28), $|\operatorname{Hess} r|^{2} \geq(\Delta r)^{2} /(m-1)$, and from the asymptotic behaviour (1.14) and (1.42), we infer that $\Delta r$ satisfies

$$
\left\{\begin{array}{l}
(\Delta r)^{\prime}+\frac{(\Delta r)^{2}}{m-1}-(m-1) G \leq(\Delta r)^{\prime}+\frac{(\Delta r)^{2}}{m-1}+\operatorname{Ricc}(\nabla r, \nabla r) \leq 0, \\
\Delta r(s)=\frac{m-1}{r}+o(1) \quad \text { as } s \rightarrow 0^{+} .
\end{array}\right.
$$

Now, if $g$ solves (1.43), $h=(m-1) g^{\prime} / g$ is a solution of

$$
h^{\prime}+\frac{h^{2}}{m-1}-(m-1) G \geq 0,
$$

and we apply the Riccati comparison Proposition 1.13 to conclude the validity of (1.45) on $D_{o} \cap B_{R_{0}}(o)$. Next, we show that $D_{o} \subset B_{R_{0}}(o)$. A computation in normal coordinates gives

$$
\Delta r=\frac{\partial}{\partial r} \log \sqrt{g(r, \theta)},
$$

where $g(r, \theta)$ is the determinant of the metric in this coordinate system. Thus, (1.45) on $D_{o} \cap B_{R_{0}}(o)$ reads

$$
\frac{\partial}{\partial r} \log \sqrt{g(r, \theta)} \leq(m-1) \frac{h^{\prime}(r)}{h(r)} .
$$

Integrating and using the asymptotic behaviour in 0 we get, for each unit vector $\theta \in T_{o} M$

$$
\sqrt{g(r, \theta)} \leq h(r)^{m-1} \quad \forall r \in\left[0, \min \left\{c(\theta), R_{0}\right\}\right),
$$


$c(\theta)$ being the distance between $o$ and the first cut-point along the geodesic $\gamma_{\theta}$. Since $g(r, \theta)>0$ on $D_{o}$, we shall have $R_{0} \geq c(\theta)$, that is, $D_{o} \subset B_{R_{0}}$. We are left to show the weak inequality, that is,

$$
-\int_{M}\langle\nabla r, \nabla \varphi\rangle \leq(m-1) \int_{M} \frac{g^{\prime}(r)}{g(r)} \varphi \quad \forall 0 \leq \varphi \in \operatorname{Lip}_{c}(M) .
$$

Now, observe that if $\hat{g}$ solves (1.43) with the equality sign, by a Sturm type argument and the positivity of $\varphi$ we get

$$
\varphi \frac{\hat{g}^{\prime}(r)}{\hat{g}(r)} \leq \varphi \frac{g^{\prime}(r)}{g(r)} .
$$

Therefore, it is enough to show (1.49) when $g$ solves (1.43) with the equality sign. Let $E_{o}$ be the star-shaped domain of the normal coordinates in $T_{o} M$. Then, $E_{o}$ can be exhausted by an increasing family of smooth star-shaped domains $\left\{E_{j}\right\}$. Let $\Omega_{j}=\exp _{o}\left(E_{j}\right)$ and denote with $\nu_{j}$ the outward pointing unit normal to $\partial \Omega_{j}$. Note that $\bigcup_{j} \Omega_{j}$ differs from $M$ by the zero measure set cut $(o)$. Consider a decreasing sequence $\left\{\varepsilon_{j}\right\}$ converging to zero such that $B_{\varepsilon_{1}}(o) \subset D_{o}$, and set $B_{j}=B_{\varepsilon_{j}}(o)$. Then, for every $0 \leq \varphi \in \operatorname{Lip}_{c}(M)$, since $B_{j}$ is regular,

$$
\begin{aligned}
-\int_{M}\langle\nabla r, \nabla \varphi\rangle & =-\lim _{j \rightarrow+\infty} \int_{\Omega_{j} \backslash B_{j}}\langle\nabla r, \nabla \varphi\rangle \\
& =\lim _{j \rightarrow+\infty}\left[-\int_{\partial \Omega_{j}} \varphi\left\langle\nabla r, \nu_{j}\right\rangle+\int_{\partial B_{j}} \varphi+\int_{\Omega_{j} \backslash B_{j}} \varphi \Delta r\right] .
\end{aligned}
$$

Since $\Omega_{j}$ is star-shaped, $\left\langle\nabla r, \nu_{j}\right\rangle \geq 0$ on $\partial \Omega_{j}$. Letting $\varepsilon \rightarrow 0$, the integral over $\partial B_{j}$ vanishes and we deduce, using also (1.45) on $\Omega_{j} \backslash B_{j} \subset D_{o}$,

$$
-\int_{M}\langle\nabla r, \nabla \varphi\rangle \leq \limsup _{j \rightarrow+\infty} \int_{\Omega_{j} \backslash B_{j}} \varphi \Delta r \leq(m-1) \limsup _{j \rightarrow+\infty} \int_{\Omega_{j} \backslash B_{j}} \frac{g^{\prime}(r)}{g(r)} \varphi .
$$

Since $g^{\prime} / g \sim 1 / r$ as $r \rightarrow 0$, the singularity in $r=0$ is integrable. It remains to show that the limit of the RHS exists. This requires a little care. We define

$$
U_{j}=\left\{x \in \Omega_{j} \backslash B_{j}: g^{\prime}(r(x)) \geq 0\right\}, \quad V_{j}=\left\{x \in \Omega_{j} \backslash B_{j}: g^{\prime}(r(x))<0\right\},
$$

And we note that both $\left\{U_{j}\right\}$ and $\left\{V_{j}\right\}$ are increasing sequences. We split the RHS as the sum of an integral over $U_{j}$ and an integral over $V_{j}$. Clearly, by the monotone converge theorem, both integrals have a limit as $r \rightarrow+\infty$. Thus, it is enough to show that the integral over $U_{j}$ has a finite limit. Let $B_{R}$ be a geodesic ball containing $\operatorname{supp} \varphi$, and let $B>0$ be sufficiently large that $G(r) \geq-B^{2}$ on $B_{R}$. We consider the function

$$
\widetilde{g}(r)=\frac{1}{B} \sinh (B r), \quad \text { which solves } \quad\left\{\begin{array}{l}
\widetilde{g}^{\prime \prime}-B^{2} \widetilde{g}=0 \\
\widetilde{g}(0)=0, \quad \widetilde{g}^{\prime}(0)=1 .
\end{array}\right.
$$

By Sturm argument we get

$$
\frac{g^{\prime}(r)}{g(r)} \leq B \operatorname{coth}(B r) \quad \text { on } \quad(0, R)
$$

hence

$$
\int_{U_{j}} \frac{g^{\prime}(r)}{g(r)} \varphi \leq B \int_{U_{j}} \varphi \operatorname{coth}(B r) \leq B \int_{M} \varphi \operatorname{coth}(B r)<+\infty
$$


Concluding,

$$
-\int_{M}\langle\nabla r, \nabla \varphi\rangle \leq(m-1) \lim _{j \rightarrow+\infty} \int_{\Omega_{j} \backslash B_{j}} \frac{g^{\prime}(r)}{g(r)} \varphi=(m-1) \int_{M} \frac{g^{\prime}(r)}{g(r)} \varphi,
$$

and the theorem is proved.

REMARK 1.20. The analytic approach for the Hessian and the Laplacian comparison theorems is extremely flexible and can be easily adapted to the more general diffusion type operator

$$
L_{D} u=\frac{1}{D} \operatorname{div}(D \nabla u) \quad 0<D \in C^{2}(M), \quad u \in C^{2}(M)
$$

on weighted manifolds $(M,\langle\rangle,, D \mathrm{~d} V)$. In this situation, the interplay with geometry is described through lower bounds on the modified Bakry-Emery Ricci tensor, which allows to prove a comparison result for $L_{D} r$ analogous to that of Theorem 1.19. There is, nevertheless, a subtle difference with the case of the Laplacian. Indeed, the asymptotic $\Delta r \sim(m-1) / r+o(1)$ as $r \rightarrow 0$ is trivially replaced with

$$
L_{D} r \frac{m-1}{r}+O(1),
$$

but the Riccati inequality analogous to (1.47) is

$$
\left(L_{D} r\right)^{\prime}+\frac{\left(L_{D} r\right)^{2}}{n-1}-(n-1) G \leq 0,
$$

for some $n>m$ coming from the definition of the modified Bakry-Emery Ricci tensor (see [MRS10] for details). A solution of (1.51) with the equality sign is $h=(n-1) g^{\prime} / g$, where $g$ solves $g^{\prime \prime}-G g=0, g(0)=0, g^{\prime}(0)=1$. Clearly,

$$
h(r) \sim \frac{n-1}{r}+o(1) \quad \text { as } r \rightarrow 0^{+} .
$$

However, the Riccati comparison Proposition 1.13 can be applied with

$$
n-1=\alpha=\beta_{2}>\beta_{1}=m-1,
$$

and the rest follows the same lines as those described above. Although, in many instances, the next results can be generalized to include diffusion type operators, to avoid unessential technicalities no further consideration will be made. The interested reader can consult the recent [MRS10], Section 2, and the references therein.

Due to the important role played by the solutions $g(r)$ of $g^{\prime \prime}-G g=0$, we need some sufficient condition to guarantee that $g>0$ on $\mathbb{R}^{+}$. The next proposition is a sharpened version of a criterion due to A. Kneser, see [Car92], p.241, and will be proved in Remark 4.8 and generalized in Theorem 5.44.

Proposition 1.21. Let $G \in C^{0}\left(\mathbb{R}_{0}^{+}\right)$be such that

$$
G_{-} \in L^{1}\left(\mathbb{R}^{+}\right), \quad s \int_{s}^{+\infty} G_{-}(\sigma) \mathrm{d} \sigma \leq \frac{1}{4} \quad \text { on } \mathbb{R}^{+} .
$$

Then, every solution of

$$
\left\{\begin{array}{l}
g^{\prime \prime}-G g \geq 0 \quad \text { on } \mathbb{R}_{0}^{+}, \\
g(0)=0, \quad g^{\prime}(0)=1
\end{array}\right.
$$


is positive and increasing on $\mathbb{R}^{+}$. If furthermore

$$
G(s) \geq-\frac{1}{4 s^{2}} \quad \text { on } \mathbb{R}^{+},
$$

then $g(s) \geq C \sqrt{s} \log s$ on $\left[s_{1},+\infty\right)$, for some $s_{1}>0$ and some positive constant $C=C\left(s_{1}\right)$.

REMARK 1.22. Hereafter, the next example will be repeatedly used. For every $B \in[0,1 / 2]$, the Cauchy problem associated to the Euler equation

$$
\left\{\begin{array}{l}
g^{\prime \prime}+\frac{B^{2}}{(1+s)^{2}} g=0 \\
g(0)=0, \quad g^{\prime}(0)=1
\end{array}\right.
$$

has the explicit, positive solution

$$
g(s)= \begin{cases}\sqrt{1+s} \log (1+s) & \text { if } B=1 / 2 ; \\ \frac{1}{\sqrt{1-4 B^{2}}}\left((1+s)^{B^{\prime \prime}}-(1+s)^{1-B^{\prime \prime}}\right) & \text { if } B \in[0,1 / 2),\end{cases}
$$

where

$$
B^{\prime \prime}=\frac{1+\sqrt{1-4 B^{2}}}{2} \in(1 / 2,1]
$$

(see also [Swa68], p.45). For $B=1 / 2$, this example shows that, under assumption (1.54), the inequality $g(s) \geq C \sqrt{s} \log s$ is sharp.

An application of the above Proposition and of the Laplacian comparison Theorem 1.17 yields the following

Corollary 1.23. Let $(M,\langle\rangle$,$) be a complete, non-compact Riemannian man-$ ifold with a pole $o$ and radial sectional curvature satisfying

$$
K_{\text {rad }}(x) \leq-G(r(x)) \quad \text { on } M \backslash\{o\},
$$

where $G \in C^{0}\left(\mathbb{R}_{0}^{+}\right)$is such that

$$
G_{-} \in L^{1}(+\infty), \quad \text { and } \quad r \int_{r}^{+\infty} G_{-}(\sigma) \mathrm{d} \sigma \leq \frac{1}{4} \quad \text { on } \quad \mathbb{R}_{0}^{+} .
$$

Then, $\Delta r>0$ on $M \backslash\{o\}$.

Integrating the Laplacian comparison inequalities from below and above we obtain the Bishop-Gromov volume comparisons. We state the estimate from above.

THEOREM 1.24. In the notations of Theorem 1.15, assume that the radial Ricci curvature satisfies

$$
\operatorname{Ricc}(\nabla r, \nabla r)(x) \geq-(m-1) G(r(x)) \quad \text { on } D_{o},
$$

for some function $G \in C^{0}\left(\mathbb{R}_{0}^{+}\right)$, and let $g \in C^{2}\left(\mathbb{R}_{0}^{+}\right)$be a solution of

$$
\left\{\begin{array}{l}
g^{\prime \prime}-G g \geq 0 \\
g(0)=0, \quad g^{\prime}(0)=1,
\end{array}\right.
$$

positive on some maximal interval $\left(0, R_{0}\right)$. Then, the functions

$$
r \longmapsto \frac{\operatorname{vol}\left(\partial B_{r}\right)}{g(r)^{m-1}}
$$


and

$$
r \longmapsto \frac{\operatorname{vol}\left(B_{r}\right)}{\int_{0}^{r} g(s)^{m-1} \mathrm{~d} s}
$$

are non-increasing a.e, respectively non-increasing, on $\left(0, R_{0}\right)$, and

$$
\operatorname{vol}\left(\partial B_{r}\right) \leq \omega_{m-1} g(r)^{m-1}, \quad \operatorname{vol}\left(B_{r}\right) \leq \omega_{m-1} \int_{0}^{r} g(s)^{m-1} \mathrm{~d} s
$$

on $\left(0, R_{0}\right)$, where $\omega_{m-1}$ is the volume of the unit $(m-1)$-sphere in $\mathbb{R}^{m}$.

Proof. We fix $0<r<R<R_{0}$. For any $\varepsilon>0$, we apply inequality (1.49) to the radial cut-off function

$$
\varphi_{\varepsilon}(x)=\rho_{\varepsilon}(r(x)) g(r(x))^{-m+1}
$$

where $\rho_{\varepsilon}$ is the piecewise linear function

$$
\rho_{\varepsilon}(s)= \begin{cases}0 & \text { if } s \in[0, r) \\ (s-r) / \varepsilon & \text { if } s \in[r, r+\varepsilon) \\ 1 & \text { if } s \in[r+\varepsilon, R-\varepsilon) \\ (R-s) / \varepsilon & \text { if } s \in[R-\varepsilon, R) \\ 0 & \text { if } s \in[R, \infty) .\end{cases}
$$

Simplifying, we get

$$
\frac{1}{\varepsilon} \int_{B_{R} \backslash B_{R-\varepsilon}} g(r(x))^{-m+1} \leq \frac{1}{\varepsilon} \int_{B_{r+\varepsilon} \backslash B_{r}} g(r(x))^{-m+1} .
$$

Using the co-area formula we deduce that

$$
\frac{1}{\varepsilon} \int_{R-\varepsilon}^{R} \operatorname{vol}\left(\partial B_{s}\right) g(s)^{-m+1} \mathrm{~d} s \leq \frac{1}{\varepsilon} \int_{r}^{r+\varepsilon} \operatorname{vol}\left(\partial B_{s}\right) g(s)^{-m+1} \mathrm{~d} s
$$

and, letting $\varepsilon \rightarrow 0$,

$$
\frac{\operatorname{vol}\left(\partial B_{R}\right)}{g(R)^{m-1}} \leq \frac{\operatorname{vol}\left(\partial B_{r}\right)}{g(r)^{m-1}}
$$

for a.e. $0<r<R<R_{0}$. Statement (1.58) follows from the first and the coarea formula, since, as observed in Section 4 of [CGT82], for general real valued functions $f(r) \geq 0, g(r)>0$,

$$
\text { if } \quad r \rightarrow \frac{f(r)}{g(r)} \quad \text { is decreasing, then } \quad r \rightarrow \frac{\int_{0}^{r} f}{\int_{0}^{r} g} \quad \text { is decreasing. }
$$

Integrating the asymptotic $\Delta r \sim(m-1) / r+o(1)$ on $\partial B_{r}$ we deduce

$$
\operatorname{vol}\left(\partial B_{r}\right) \sim \omega_{m-1} r^{m-1}
$$

which, together with (1.57), proves (1.59).

As the above proof and Theorem 1.17 show, the control from below on $\operatorname{vol}\left(\partial B_{r}\right)$ and the related reversed monotonicity formula require an upper bound on the radial sectional curvatures and are valid only for regular geodesic balls, that is, geodesic balls contained in the domain of normal coordinates. For particular $G(r)$, explicit solutions $g$ of (1.43) can be provided, and will be repeatedly used in the next sections. The reader can find such $g$ 's in the proof of Theorems 3.16 and 3.18. For 
this reason, here we limit ourselves to state the estimates with no proof. For the case $\alpha=-2$ of the upper bounds, we suggest the reader to consult also [CGT82], Theorem 4.9 .

Proposition 1.25 ([PRS08], Proposition 2.1). Let $(M,\langle\rangle$,$) be a complete$ Riemannian manifold of dimension $m \geq 2$, with radial Ricci curvature satisfying

$$
\operatorname{Ricc}(\nabla r, \nabla r)(x) \geq-(m-1)^{2} B^{2}\left(1+r(x)^{2}\right)^{\alpha / 2} \quad \text { on } D_{o},
$$

for some $B>0, \alpha \geq-2$. Then, for $r \geq 1$ there exists a constant $C>0$ such that

$$
\operatorname{vol}\left(\partial B_{r}\right) \leq C \begin{cases}\exp \left\{\frac{2 B(m-1)}{2+\alpha}(1+r)^{1+\frac{\alpha}{2}}\right\} & \text { if } \alpha \geq 0 ; \\ r^{-\frac{\alpha(m-1)}{4} \exp \left\{\frac{2 B(m-1)}{2+\alpha} r^{1+\frac{\alpha}{2}}\right\}} & \text { if } \alpha \in(-2,0) ; \\ r^{(m-1) B^{\prime}} & \text { if } \alpha=-2,\end{cases}
$$

Where $B^{\prime}=\left(1+\sqrt{1+4 B^{2}}\right) / 2$.

Proposition 1.26. Let $(M,\langle\rangle$,$) be a complete Riemannian manifold of di-$ mension $m \geq 2$, with a pole o and radial sectional curvature satisfying

$$
K_{\mathrm{rad}}(x) \leq-B^{2}\left(1+r(x)^{2}\right)^{\alpha / 2} \quad \text { on } M \backslash\{o\},
$$

for some $B>0, \alpha \geq-2$. Then, for $r \geq 1$ there exists a constant $C>0$ such that

$$
\operatorname{vol}\left(\partial B_{r}\right) \geq C \begin{cases}r^{-\frac{\alpha(m-1)}{4} \exp \left\{\frac{2 B(m-1)}{2+\alpha} r^{1+\frac{\alpha}{2}}\right\}} & \text { if } \alpha \geq 0 ; \\ \exp \left\{\frac{2 B(m-1)}{2+\alpha}(1+r)^{1+\frac{\alpha}{2}}\right\} & \text { if } \alpha \in(-2,0) ; \\ r^{(m-1) B^{\prime}} & \text { if } \alpha=-2,\end{cases}
$$

where $B^{\prime}=\left(1+\sqrt{1+4 B^{2}}\right) / 2$.

By using the solutions $g$ described in Remark 1.22, we can easily state volume comparison theorems under curvature bounds of the type

$$
\operatorname{Ricc}(\nabla r, \nabla r)(x) \geq(m-1) \frac{B^{2}}{(1+r(x))^{2}}, \quad \text { resp. } \quad K_{\mathrm{rad}}(x) \leq \frac{B^{2}}{(1+r(x))^{2}},
$$

for some $B \in(0,1 / 2]$. The reason of the appearance of the constant $1 / 2$ will be clarified in later sections.

\subsection{Some spectral theory on manifolds}

Since in the sequel we will be concerned with spectral arguments for some elliptic operators, we recall a few constructions and results. We assume that the reader is familiar with the basics of spectral theory on Hilbert spaces, for which we refer to the book of T. Kato [Kat95] and to the encyclopedic treatise of M. Reed and B. Simon, especially [RS80], Chapter VIII and [RS75], Chapter X. The main source for this section is the concise but detailed account in [PRS08], Section 3, and we suggest the reader to consult the references therein for further insight. Let $(M,\langle\rangle$,$) be a Riemannian manifold; let A: \Gamma(T M) \rightarrow \Gamma(T M)$ be a symmetric endomorphism such that $A$ is positive definite at every point of $M$, and let $q(x) \in L_{\mathrm{loc}}^{\infty}(M)$. The regularity $A \in C_{\mathrm{loc}}^{1, \alpha}$, for some $\alpha \in(0,1)$, suffices for our 
purposes. However, in our applications $A$ will always be smooth. In what follows, we shall be concerned with complex vector fields, and we agree on using the same symbol $A$ to denote also the quadratic form defined by $A(X, Y)=\langle A X, Y\rangle$ for each $X, Y \in \Gamma\left(T M^{\mathbb{C}}\right)$. Consider the differential operator $L: C_{c}^{\infty}(M) \rightarrow C_{c}^{\infty}(M)$ given by

$$
L u=-\operatorname{div}(A \nabla u)-q(x) u \quad \forall u \in C_{c}^{\infty}(M) .
$$

For convenience, we shall think of $L$ as acting on complex-valued functions. Since $A$ is symmetric, $L$ is a symmetric linear operator on $L^{2}(M)$ endowed with the inner product

$$
(u, v)_{L^{2}}=\int_{M} u \bar{v}, \quad \forall u, v \in L^{2}(M),
$$

where integration is with respect to the volume measure. Thus, by standard spectral theory, $L$ is closable. Denote with $L^{*}$ its adjoint, which by construction is closed on its domain

$$
\mathcal{D}\left(L^{*}\right)=\left\{u \in L^{2}(M): L u \in L^{2}(M) \text { as a distribution }\right\} .
$$

By elliptic regularity of ultra-weak solutions (see $[\mathbf{A g m 1 0}]$ ), if $u \in \mathcal{D}\left(L^{*}\right)$ then $u \in H_{\mathrm{loc}}^{2}(M)$, so that

$$
\mathcal{D}\left(L^{*}\right)=\left\{u \in H_{\mathrm{loc}}^{2}(M) \cap L^{2}(M): L u \in L^{2}(M)\right\} .
$$

Since $A$ is real on real vector fields, it is easy to deduce that $u \in \operatorname{ker}\left(L^{*}+i\right)$ if and only if $\bar{u} \in \operatorname{ker}\left(L^{*}-i\right)$. This shows that the deficiency indices $d_{ \pm}=\operatorname{dim} \operatorname{ker}\left(L^{*} \pm\right.$ $i)$ are equal, thus by spectral theory $L$ always admits at least one self-adjoint extension. We recall that the self-adjoint extension is unique if and only if $L$ is essentially self-adjoint, equivalently if $L^{*}=L^{* *}$, and in this case $L^{*}$ is the selfadjoint extension of $L$.

Let $\Omega$ be any open, relatively compact domain of $M$ with Lipschitz boundary, and define $L_{\mid \Omega}$ as the operator $L$ on $C_{c}^{\infty}(\Omega)$. Indeed, Lipschitz regularity of the boundary is basically required in order to have the validity of the RellichKondrachov compactness theorem, see [EG92]. As in (1.67),

$$
\mathcal{D}\left(L_{\mid \Omega}^{*}\right)=\left\{u \in H_{\mathrm{loc}}^{2}(\Omega) \cap L^{2}(\Omega): L u \in L^{2}(\Omega) \text { as a distribution }\right\} .
$$

From $\left(L_{\mid \Omega} u, u\right) \geq-\|q\|_{L^{\infty}(\Omega)}\|u\|_{L^{2}}, L_{\mid \Omega}$ is bounded from below. The quadratic form associated to $L_{\mid \Omega}$ is

$$
\begin{array}{ccc}
Q_{\mid \Omega}: C_{c}^{\infty}(\Omega) \times C_{c}^{\infty}(\Omega) & \longrightarrow & \mathbb{C} \\
u, v & \longrightarrow & (L u, v)_{L^{2}}=\int_{\Omega}[A(\nabla u, \nabla \bar{v})+q u \bar{v}] .
\end{array}
$$

Since $q \in L_{\mathrm{loc}}^{\infty}$ and $A$ is locally equivalent to $\langle$,$\rangle , there exist positive constants$ $C_{1}, \widetilde{C}_{1}, C_{2}, \widetilde{C}_{2}$ such that

$$
C_{1}\|u\|_{H^{1}}^{2} \leq \widetilde{C}_{1}\|\nabla u\|_{L^{2}}^{2} \leq Q_{\mid \Omega}(u, u)+\widetilde{C}_{2}\|u\|_{L^{2}}^{2} \leq C_{2}\|u\|_{H^{1}}^{2},
$$

where the first inequality is the Poincarè inequality on $\Omega$ ([LS84], Corollary 1.1). The norm induced by $Q_{\mid \Omega}+\widetilde{C}_{2}(,)_{L^{2}}$ is therefore the $H^{1}$ norm, whence the closure $Q_{\Omega}$ of $Q_{\mid \Omega}$ is defined on $H_{0}^{1}(\Omega) \times H_{0}^{1}(\Omega)$. By standard theory, the Friedrichs extension $L_{\Omega}$ of $L_{\mid \Omega}$ is the self-adjoint extension of $L_{\mid \Omega}$ whose domain is

$\mathcal{D}\left(L_{\Omega}\right)=\left\{u \in H_{0}^{1}(\Omega): \exists w \in L^{2}(\Omega)\right.$ satisfying $\left.Q_{\Omega}(u, \phi)=(w, \phi)_{L^{2}} \forall \phi \in H_{0}^{1}(\Omega)\right\}$, 
the image of $u \in \mathcal{D}\left(L_{\Omega}\right)$ being given by $L_{\Omega} u=w$. We can also consider the next bounded, $\mathbb{C}$-linear operator:

$$
T_{\Omega} \quad: \quad\left(H_{0}^{1}(\Omega),\|\cdot\|_{H^{1}}\right) \longrightarrow H^{-1}(\Omega), \quad \text { by setting } \quad T_{\Omega} u=Q_{\Omega}(u, \cdot),
$$

$H^{-1}(\Omega)$ being the dual of $H_{0}^{1}(\Omega)$ endowed with its operator norm. This is called the weak extension of $L_{\mid \Omega}$, often called the extension in the sense of quadratic forms. By (1.70), $T_{\Omega}$ applied to $u \in \mathcal{D}\left(L_{\Omega}\right)$ gives rise to the functional $\left(L_{\Omega} u, \cdot\right) \in H^{-1}(\Omega)$, whence $u \in H_{0}^{1}(\Omega)$ belongs to $\mathcal{D}\left(L_{\Omega}\right)$ if and only if $T_{\Omega} u \in L^{2}(\Omega) \leq H^{-1}(\Omega)$. If $\lambda \in$ $\mathbb{R}$ is sufficiently large, $Q_{\Omega}+\lambda(,)_{L^{2}}$ is continuous and coercive on $H_{0}^{1}(\Omega) \times H_{0}^{1}(\Omega)$. Lax-Milgram theorem gives that

$$
Q_{\Omega}(u, \cdot)+\lambda(u, \cdot)_{L^{2}}=<f, \cdot>,
$$

as an equality in $H^{-1}(\Omega)$, has a unique solution $u \in H_{0}^{1}(\Omega)$ for each fixed $f \in$ $H^{-1}(\Omega)$. Combining (1.71) and (1.72), by the open mapping theorem the operator

$$
T_{\Omega}+\lambda \quad: \quad\left(H_{0}^{1}(\Omega),\|\cdot\|_{H^{1}}\right) \longrightarrow H^{-1}(\Omega)
$$

is a $\mathbb{C}$-linear homeomorphism. Therefore,

$$
L^{2}(\Omega) \hookrightarrow H^{-1}(\Omega) \stackrel{\left(T_{\Omega}+\lambda\right)^{-1}}{\longrightarrow} H_{0}^{1}(\Omega) \hookrightarrow L^{2}(\Omega)
$$

is a compact map, being the composition of continuous maps with the inclusion

$$
\left(H_{0}^{1}(\Omega),\|\cdot\|_{H^{1}}\right) \hookrightarrow L^{2}(\Omega),
$$

which is compact by Rellich-Kondrachov theorem (here the requirement $\partial \Omega$ being of Lipschitz class is essential, see [EG92], Section 4.6). We still denote (1.73) with $\left(T_{\Omega}+\lambda\right)^{-1}$. By the symmetry of $A,\left(T_{\Omega}+\lambda\right)^{-1}$ is self-adjoint, and the spectral theorem gives the existence of a sequence of (positive) eigenvalues $\left\{\sigma_{k}^{-1}\right\}$, each counted with its finite multiplicity, such that $\sigma_{k}^{-1} \rightarrow 0^{+}$. If $\left\{u_{k}\right\} \subset L^{2}(\Omega)$ is the corresponding complete orthonormal set of eigenfunctions in $L^{2}(\Omega)$,

$$
\left(T_{\Omega}+\lambda\right)^{-1} u_{k}=\sigma_{k}^{-1} u_{k}, \quad \text { that is, } \quad\left(T_{\Omega}+\lambda\right) u_{k}=\sigma_{k} u_{k} .
$$

Setting $\lambda_{k}^{L}(\Omega)=\sigma_{k}-\lambda$, we have thus

$$
T_{\Omega} u_{k}=\lambda_{k}^{L}(\Omega) u_{k} \in L^{2}(\Omega) \leq H^{-1}(\Omega)
$$

in the sense of quadratic forms. By definition, $\left\{u_{k}\right\} \subset \mathcal{D}\left(L_{\Omega}\right)$, and (1.74) also implies the equality $L_{\mid \Omega} u_{k}=\lambda_{k}^{L}(\Omega) u_{k}$ in the distributional sense, as we have

$$
<L_{\mid \Omega} u_{k}, \phi>=\left(u_{k}, L_{\mid \Omega} \phi\right)_{L^{2}}=Q_{\Omega}\left(u_{k}, \phi\right)=\lambda_{k}^{L}(\Omega)\left(u_{k}, \phi\right)_{L^{2}}
$$

for each $\phi \in C_{c}^{\infty}(\Omega)$, where the first equality is by definition, and the second one follows integrating by parts. Combining with (1.68) we deduce that $\left\{u_{k}\right\} \subset \mathcal{D}\left(L_{\mid \Omega}^{*}\right)$, whence $\mathcal{D}\left(L_{\mid \Omega}^{*}\right)$ contains an $L^{2}$ orthonormal basis made up of eigenfunctions. By Theorem 3.2 of [PRS08], $L_{\mid \Omega}^{*}$ is essentially self-adjoint on its domain. Being $L_{\mid \Omega}^{*}$ closed by construction, $L_{\mid \Omega}^{*}=L_{\mid \Omega}^{* *}$ is self-adjoint, or equivalently $\left(L_{\mid \Omega}, C_{c}^{\infty}(\Omega)\right)$ is essentially self-adjoint. Since $L_{\Omega}^{* *}$ is the closure of $\left(L_{\Omega}, C_{c}^{\infty}(\Omega)\right)$ in the graph norm, we can say that

(1.77)

$\forall u \in \mathcal{D}\left(L_{\Omega}^{*}\right) \quad \exists\left\{u_{j}\right\} \subset C_{c}^{\infty}(\Omega) \quad$ such that $\left\|u_{j}-u\right\|_{L^{2}} \rightarrow 0, \quad\left\|L u_{j}-L u\right\|_{L^{2}} \rightarrow 0$

as $j \rightarrow+\infty$. 
Remark 1.27. By Theorem 1.1 of [Tol84], $u_{k} \in C_{\text {loc }}^{1, \beta}(\Omega)$. The classical interior regularity $u_{k} \in C_{\mathrm{loc}}^{2, \alpha}(\Omega)$, for some $\alpha \in(0,1)$, is attained provided that $q \in C_{\mathrm{loc}}^{0, \alpha}(\Omega)$.

Summarizing, for relatively compact, open sets $\Omega$ with Lipschitz boundary $L_{\Omega} \equiv L_{\mid \Omega}^{*}$ is the unique self-adjoint extension of $L_{\mid \Omega}$ and, again by Theorem 3.2 of [PRS08], its spectrum consists of the divergent sequence $\left\{\lambda_{k}^{L}(\Omega)\right\}$. An applications of the Poincaré-Polya min-max theorem (see [PRS08], Theorem 3.7 and [Dav95], Theorems 4.5.1, 4.5.2, 4.5.3) gives the characterization

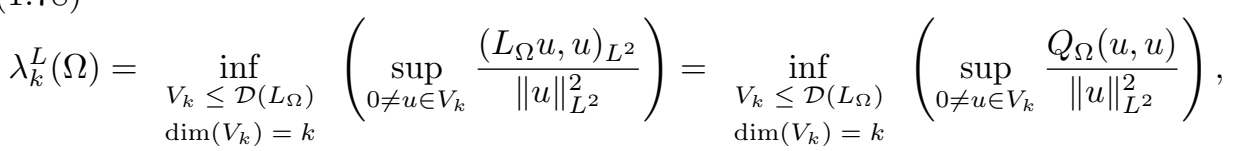

where the last equality follows from the construction of $L_{\Omega}$, and where $\mathcal{D}\left(L_{\Omega}\right)$ can be substituted by any core for the quadratic form $Q_{\Omega}$, that is, every dense subspace of $\left(H_{0}^{1}(\Omega),\|\cdot\|_{H^{1}}\right)$. In particular, we can use $C_{c}^{\infty}(\Omega), \operatorname{Lip}_{0}(\Omega), H_{0}^{1}(\Omega)$. Splitting into real and imaginary parts, it is easy to see that, in (1.78), we can restrict ourselves to consider real-valued functions $u$. It is worth to point out that there is also a complementary max-min principle for $\lambda_{k}^{L}(\Omega)$, originating from the works of H. Weyl and of R. Courant, D. Hilbert. The relationship between the min-max and the max-min characterizations, together with historical references, is worked out in detail, for instance, in the paper of W. Stenger [Ste70].

We conclude this short account for $L_{\Omega}$ by remarking that each stationary point $u \in H_{0}^{1}(\Omega)$ of the functional $\phi \mapsto Q_{\Omega}(\phi, \phi)$ satisfies the Euler-Lagrange equations

$$
\left(Q_{\Omega}-\lambda_{1}^{L}(\Omega)(,)\right)(u, \phi)=0 \quad \text { for every } \phi \in H_{0}^{1}(\Omega),
$$

that is, from (1.71), $u$ must be a weak solution of $L_{\Omega} u=\lambda_{1}^{L}(\Omega) u$ (this is classically called Courant minimum principle).

REMARK 1.28. Let $u$ be a real valued eigenfunction of $L$ relative to the first eigenvalue $\lambda_{1}^{L}(\Omega)$. As we have observed, $u \in C_{\text {loc }}^{1, \beta}(\Omega) \cap H_{0}^{1}(\Omega)$. It is well known that $u$ has constant sign on $\Omega$, and thus $\lambda_{1}^{L}(\Omega)$ is a simple eigenvalue. We briefly recall how to prove that. Assume by contradiction that $u$ changes sign on $\Omega$. Then, $u_{+}$and $u_{-}$are nonzero $\operatorname{Lip}_{0}(\Omega)$ functions, each vanishing on some open subset of $\Omega$. Applying the weak definition of $L u=\lambda_{1}^{L}(\Omega) u$ to the test functions $u_{+}$, and using the min-max definition of $\lambda_{1}^{L}(\Omega)$ we get

$$
0=\left(Q_{\Omega}-\lambda_{1}^{L}(\Omega)(,)\right)\left(u, u_{+}\right) \equiv\left(Q_{\Omega}-\lambda_{1}^{L}(\Omega)(,)\right)\left(u_{+}, u_{+}\right) \geq 0,
$$

thus $u_{+}$is a minimum of the Rayleigh quotient. Analogously, we can prove that also $u_{-}$is a minimum. Hence, by Courant minimum principle $u_{+}$and $u_{-}$are both eigenfunctions, each vanishing on some nonempty open subset of $\Omega$. This contradicts the unique continuation property ([Aro57] and [PRS08], Appendix A). Up to changing the sign, this shows that $u \geq 0$ on $\Omega$. The stronger $u>0$ follows from the strong maximum principle, see [GT98], p.35. As a consequence, $\lambda_{1}^{L}(\Omega)$ is a simple eigenvalue, for if not there should be a plane $\pi \subset L^{2}(\Omega)$ consisting of eigenfunctions for $\lambda_{1}^{L}(\Omega)$, and we could find an eigenfunction $u_{2} \perp u$, which is impossible since both $u, u_{2}$ have constant sign. The interested reader should consult Chapter 1 of [Cha84] for related discussions. 
By the domain monotonicity of eigenvalues (see [Sma65], [Sma67]) or, as well, by the unique continuation property ([Aro57] and [PRS08], Appendix A), $\lambda_{k}^{L}(\Omega) \geq \lambda_{k}^{L}\left(\Omega^{\prime}\right)$ whenever $\Omega \subset \Omega^{\prime}$, and strict inequality holds if $\Omega^{\prime} \backslash \Omega$ has nonempty interior.

We define the index of $L_{\Omega}, \operatorname{ind}_{L}(\Omega)$, as

$$
\operatorname{ind}_{L}(\Omega)=\sup \left\{\operatorname{dim} V: \begin{array}{l}
V \leq \mathcal{D}\left(L_{\Omega}\right), \quad \operatorname{dim} V<\infty, \\
\left(L_{\Omega} u, u\right)_{L^{2}}<0 \quad \forall 0 \neq u \in V
\end{array}\right\},
$$

and we observe that we can substitute $V \leq \mathcal{D}\left(L_{\Omega}\right)$ with subspaces $V$ of any core for $Q_{\Omega}$ contained in $\mathcal{D}\left(L_{\Omega}\right)$. By the previous discussion, $\operatorname{ind}_{L}(\Omega)$ coincides with the number of negative eigenvalues, thus $\operatorname{ind}_{L}(\Omega)<\infty$ and increases when $\Omega$ grows.

We now turn to the description of $L$ on $C_{c}^{\infty}(M)$. First, the essential selfadjointness of $\left(L, C_{c}^{\infty}(M)\right)$ is not automatic. Suppose that $L$ is bounded from below on $C_{c}^{\infty}(M)$, that is, there exists $c \in \mathbb{R}$ such that

$$
(L u, u)_{L^{2}} \geq c\|u\|_{L^{2}}^{2} \quad \forall u \in C_{c}^{\infty}(M) .
$$

Then, we have the next sufficient condition for complete Riemannian manifolds, compare also with $[$ Che73], [Kat74] and [Str83].

Proposition 1.29. Let $M$ be a complete Riemannian manifold, and let $r(x)$ be the distance function from a fixed origin o. Assume that $L$ is bounded from below on $C_{c}^{\infty}(M)$ and that

$$
\liminf _{r \rightarrow+\infty} \frac{\|A\|_{L^{\infty}\left(B_{r}\right)}}{r}<+\infty, \quad \text { where } \quad\|A\|_{L^{\infty}\left(B_{r}\right)}=\sup _{x \in B_{r}}|A|(x)
$$

and $|A|(x)$ is the Hilbert-Schmidt norm of $A$ at $x$. Then, $\left(L, C_{c}^{\infty}(M)\right)$ is essentially self-adjoint.

Remark 1.30. The above Proposition can be obtained, with minor modifications, from Theorem 3.12 of [PRS08]. Indeed, the requirement (1.81) allows to follow the proof step by step up to the desired conclusion. Se also the recent [Mar] for a mild generalization.

Remark 1.31. Note that (1.81) is met, for instance, when $A \equiv \mathrm{Id}$, that is, for the Schrödinger operator $L=-\Delta-q(x)$. For the sake of completeness, even if we do not address the problem here, we mention that the essential self-adjointness of $-\Delta-q(x)$ on $C_{c}^{\infty}(M)$, when $q$ has well-behaved singularities, has been proved for instance in [DG97] and [Agm85].

In general, for arbitrary non-compact Riemannian manifolds $L$ may fail to be essentially self-adjoint on $C_{c}^{\infty}(M)$, even when $L$ is bounded from below. In this case, $L$ has infinitely many self-adjoint extensions. In what follows, when $L$ is bounded from below, we agree on considering always the Friedrichs extension $L_{M}$, that is, the self-adjoint extension of $L$ associated to the closure of the quadratic form

$$
\begin{array}{ccc}
Q: C_{c}^{\infty}(M) \times C_{c}^{\infty}(M) & \longrightarrow & \mathbb{C} \\
u, v & \longrightarrow(L u, v)_{L^{2}}=\int_{M}[A(\nabla u, \nabla \bar{v})+q u \bar{v}],
\end{array}
$$


with respect to the norm $\|\cdot\|_{Q}$ induced by the inner product $Q+(1-c)(,)_{L^{2}}$ on $C_{c}^{\infty}(M)$, where $c$ is as in (1.80). It turns out that

$\mathcal{D}\left(L_{M}\right)=\left\{u \in{\overline{C_{c}^{\infty}(M)}}^{\|\cdot\|_{Q}}: \exists w \in L^{2}(M)\right.$ s.t. $\left.Q(u, \phi)=(w, \phi)_{L^{2}} \forall \phi \in C_{c}^{\infty}(M)\right\}$,

For such $u, L_{M} u=w$.

REMARK 1.32. From (1.83) it is easy to see that $\mathcal{D}\left(L_{M}\right) \subset H_{\mathrm{loc}}^{2}(M)$. Indeed, fix any $\Omega \Subset U \Subset M$ and $\psi \in C_{c}^{\infty}(U)$ such that $\psi=1$ on $\Omega$. For $u \in \mathcal{D}\left(L_{M}\right)$, clearly $\psi u \in \mathcal{D}\left(L_{U}\right)$. Since $L$ is essentially self-adjoint on $C_{c}^{\infty}(U), \mathcal{D}\left(L_{U}\right) \equiv \mathcal{D}\left(L_{\mid U}^{*}\right)$, hence by the definition (1.68) and regularity of distributional solutions, $\psi u \in H_{\mathrm{loc}}^{2}(U)$, thus $u \in H^{2}(\Omega)$. By the arbitrariness of $\Omega, u \in H_{\mathrm{loc}}^{2}(M)$, as claimed.

When $L$ is bounded from below on $C_{c}^{\infty}(M)$, the min-max prnciple ([PRS08], Theorem 3.7) can be applied to give the variational characterization of the discrete part below the bottom of the essential spectrum $\sigma_{\text {ess }}\left(L_{M}\right)$. Having defined

$$
\lambda_{k}^{L}(M)=\inf _{\substack{V_{k} \leq \mathcal{D}\left(L_{M}\right) \\ \operatorname{dim}\left(V_{k}\right)=k}}\left(\sup _{\substack{0 \neq u \in V_{k}\\}} \frac{Q(u, u)}{\|u\|_{L^{2}}^{2}}\right),
$$

one of the following three cases occur:

- $\sigma_{\text {ess }}\left(L_{M}\right)=\emptyset$. In this case, $\left\{\lambda_{k}^{L}(M)\right\}$ consists of all the eigenvalues of $L_{M}$, written in increasing order and repeated according to multiplicity, and $\lambda_{k}^{L}(M) \rightarrow+\infty$ as $k \rightarrow+\infty$;

- $\sigma_{\text {ess }}\left(L_{M}\right) \neq \emptyset$, and there exists $C \in \mathbb{R}$ such that $\lambda_{k}^{L}(M)<C$ for every $k$ and $\lambda_{k}^{L}(M) \rightarrow C$. Then, inf $\sigma_{\text {ess }}\left(L_{M}\right)=C, \sigma\left(L_{M}\right) \cap(-\infty, C)=\left\{\lambda_{k}^{L}(M)\right\}$ and each $\lambda_{k}^{L}(M)$ is an eigenvalue;

- $\sigma_{\text {ess }}\left(L_{M}\right) \neq \emptyset$, and there exists $C \in \mathbb{R}$ such that $\lambda_{k}^{L}(M)<C$ for every $k \in\{1, \ldots, N\}$ and $\lambda_{k}^{L}(M)=C$ for every $k>N$. Then, $\inf \sigma_{\text {ess }}\left(L_{M}\right)=C$, $\sigma\left(L_{M}\right) \cap(-\infty, C)=\left\{\lambda_{k}^{L}(M)\right\}_{k=1}^{N}$ and each $\lambda_{k}^{L}(M), 1 \leq k \leq N$, is an eigenvalue.

Again, in (1.84), $\mathcal{D}\left(L_{M}\right)$ can be substituted with $C_{c}^{\infty}(M), \operatorname{Lip}_{c}(M)$ or any other core for $Q$. As for (1.78), it is enough to evaluate the Rayleigh quotients on real-valued $u$, and for this reason hereafter we consider every function space as consisting only of real-valued functions. Combining (1.78) and the monotonicity of eigenvalues, we have

$$
\begin{aligned}
\lambda_{1}^{L}(M) & =\inf \left\{\lambda_{1}^{L}(\Omega): \Omega \subset M \text { is a relatively compact domain }\right\} \\
& =\lim _{j \rightarrow+\infty} \lambda_{1}^{L}\left(\Omega_{j}\right)
\end{aligned}
$$

where $\left\{\Omega_{j}\right\}$ is any exhaustion of $M$ by means of increasing, relatively compact domains with Lipschitz boundary. Moreover, if $\left(L, C_{c}^{\infty}(M)\right)$ is bounded from below,

$$
\lambda_{k}^{L}(M)=\lim _{j \rightarrow+\infty} \lambda_{k}^{L}\left(\Omega_{j}\right) \quad \forall k \geq 1 .
$$

Indeed, if we denote with $\delta_{k}$ the RHS of (1.85), by the min-max $\lambda_{k}^{L}(M) \leq \delta_{k}$. To prove the converse, fix $\varepsilon>0$. Since $C_{c}^{\infty}(M)$ is a core for the Friedrichs extension, there exists a subspace $V_{k} \leq C_{c}^{\infty}(M)$ such that

$$
\sup _{0 \neq u \in V_{k}} \frac{Q(u, u)}{\|u\|_{L^{2}}^{2}} \leq \lambda_{k}^{L}(M)+\varepsilon \text {. }
$$


Since $V_{k} \subset C_{c}^{\infty}(M)$ is finite dimensional, there exists a large compact set $\Omega$ such that $V_{k} \leq C_{c}^{\infty}(\Omega)$. If $j$ is sufficiently large that $\Omega \subset \Omega_{j}$, by the min-max and (1.86) we get $\lambda_{k}^{L}\left(\Omega_{j}\right) \leq \lambda_{k}^{L}(\Omega) \leq \lambda_{k}^{L}(M)+\varepsilon$, and the sought follows letting $j \rightarrow+\infty$ by the arbitrariness of $\varepsilon$.

For future use, for every subset $Z \subset M$ we define the "first eigenvalue" of $L$ on $Z$, $\lambda_{1}^{L}(Z)$ as follows:

$$
\lambda_{1}^{L}(Z)=\sup \left\{\lambda_{1}^{L}(\Omega): Z \subset \Omega \text { and } \Omega \text { is an open set }\right\} .
$$

The following theorems are taken from works of D. Fischer-Colbrie [FC85], D. Fischer-Colbrie and R. Schoen, [FCS80], W.F. Moss and J. Piepenbrink [MP78] and P. Bèrard, M.P. Do Carmo and W. Santos [BCS97], and have been collected, in a slightly generalized form, in [PRS08]. Most of the results also appeared in the paper of S. Agmon [Agm85], where further attention has been paid to the regularity of $q$. With the exception of [MP78], all the papers consider the prototype Schrödinger operator $L=-\Delta-q(x)$. However, the proofs use only local arguments and can be rephrased, verbatim, for more general elliptic operators such as, for instance, those coming from the Newton tensors of an isometrically immersed oriented hypersurface. For details, see Section 5.5, where we shall use the result below in this generality.

Theorem 1.33 ([FCS80], [MP78] and [PRS08], Lemma 3.10). Let $\Omega$ be an open set of a Riemannian manifold $(M,\langle\rangle$,$) , with possibly non-compact closure,$ and let $q \in L_{\mathrm{loc}}^{\infty}(\Omega)$. The following facts are equivalent:

(i) There exists $w \in C^{1}(\Omega), w>0$ which solves $L w=0$ weakly on $\Omega$;

(ii) There exists $w \in H_{\mathrm{loc}}^{1}(\Omega), w \geq 0, w \not \equiv 0$ which solves $L w \geq 0$ weakly on $\Omega$

(iii) $\lambda_{1}^{L}(\Omega) \geq 0$.

Definition 1.34. When $\lambda_{1}^{L}(\Omega) \geq 0$, we say that $L$ is stable on $\Omega$.

REMARK 1.35. We stress that no connectedness of $\Omega$ is required. Indeed, the domain monotonicity of eigenvalues allows us to work on each connected component separately.

Remark 1.36. Indeed, $w \in C_{\text {loc }}^{1, \beta}(\Omega)$, for some $\beta \in[0,1)$. If $q \in C_{\text {loc }}^{0, \alpha}(\Omega)$, $\alpha \in(0,1)$, then $w \in C_{\mathrm{loc}}^{2, \alpha}(\Omega)$ is a classical solution of $L w=0$.

Corollary 1.37 ([BCS97], Prop. 1 and [PRS08], Theorem 3.12). Let $(M,\langle\rangle$,$) be a Riemannian manifold. The following statements are equivalent:$

(i) $L$ is bounded from below on $C_{c}^{\infty}(M)$;

(ii) For every relatively compact open set $\Omega, L$ is bounded from below on $C_{c}^{\infty}(M \backslash \bar{\Omega})$

(iii) There exists a relatively compact set $\Omega$ such that $L$ is bounded from below on $C_{c}^{\infty}(M \backslash \bar{\Omega})$.

The following theorem is often called the decomposition principle, and originates from a work of H. Donnelly and P. Li [LD79]. The characterization of the bottom of the essential spectrum is due to A. Persson in the previous paper [Per60]. We shall remark that the proofs in $[\mathbf{B C S 9 7}]$ and $[\mathbf{P R S 0 8}]$ require not only that $L$ is bounded from below, but also that $\left(L, C_{c}^{\infty}(M)\right)$ is essentially self-adjoint. This condition, automatic if $M$ is complete and $L=-\Delta-q(x)$, turns out to be quite 
restrictive for geometrically relevant operators $L$ whose principal part is, for example, a Newton operator (see Section 5.5). Indeed, for those $L$ condition (1.81) in not automatically satisfied. However, refining the proof in [PRS08], in the recent [Mar] the author has observed that both the completeness assumption on $M$ and the essential self-adjointness of $\left(L, C_{c}^{\infty}(M)\right)$ are, in fact, unnecessary.

Theorem 1.38 ([BCS97], Prop. 2, [PRS08], Theorem 3.15 and [Mar]). Assume that $L$ is bounded from below on $C_{c}^{\infty}(M)$. Then, for every $\Omega \Subset M$,

$$
\sigma_{\text {ess }}\left(L_{M}\right) \equiv \sigma_{\text {ess }}\left(L_{M \backslash \bar{\Omega}}\right) .
$$

Moreover, for every exhaustion $\left\{\Omega_{j}\right\} \uparrow M$,

$$
\begin{aligned}
\inf \sigma_{\text {ess }}\left(L_{M}\right) & =\sup _{\Omega \Subset M}\left(\inf \sigma\left(L_{M \backslash \bar{\Omega}}\right)\right)=\sup _{\Omega \Subset M}\left(\lambda_{1}^{L}(M \backslash \bar{\Omega})\right) \\
& =\lim _{j \rightarrow+\infty}\left(\lambda_{1}^{L}\left(M \backslash \bar{\Omega}_{j}\right)\right) \\
& =\lim _{j \rightarrow+\infty}\left(\inf _{0 \neq u \in C_{c}^{\infty}\left(M \backslash \bar{\Omega}_{j}\right)} \frac{Q(u, u)}{\|u\|_{L^{2}}^{2}}\right) .
\end{aligned}
$$

The definition of the (spectral) index follows (1.79), that is, $\operatorname{ind}_{L}(M)$ is the dimension, possibly infinite, of the spectral projection $\mathbb{P}_{(-\infty, 0)} L^{2}(M)$ :

$$
\operatorname{ind}_{L}(M)=\sup \left\{\operatorname{dim} V: \begin{array}{l}
V \leq \mathcal{D}\left(L_{M}\right), \quad \operatorname{dim} V<\infty, \\
\left(L_{M} u, u\right)_{L^{2}}<0 \quad \forall 0 \neq u \in V
\end{array}\right\} .
$$

Where any core for $Q$ can replace $\mathcal{D}\left(L_{M}\right)$. Our last task is to explore the relationship between $\operatorname{ind}_{L}(M)$ and the index of $L$ on relatively compact domains. We define the Morse index, $\widetilde{\operatorname{ind}}_{L}(M)$, as

$$
\widetilde{\operatorname{ind}}_{L}(M)=\sup _{\Omega \Subset M} \operatorname{ind}_{L}(\Omega) .
$$

Clearly, by the monotonicity of eigenvalues, $\widetilde{\operatorname{ind}}_{L}(M)=\lim _{j \rightarrow+\infty} \operatorname{ind}_{L}\left(\Omega_{j}\right)$ for every exhaustion $\left\{\Omega_{j}\right\}$.

Lemma 1.39. $\operatorname{ind}_{L}(M)=\widetilde{\operatorname{ind}_{L}}(M)$, possibly with infinite values.

Proof. Let $n$ be such that $\widetilde{\operatorname{ind}}_{L}(M) \geq n$. Then, there exists $\Omega \Subset M$ such that $\operatorname{ind}_{L}(\Omega) \geq n$. Since $L_{\Omega}$ is essentially self-adjoint on $C_{c}^{\infty}(\Omega)$, we can find $V \leq$ $C_{c}^{\infty}(\Omega), \operatorname{dim} V=n$, such that $Q$ is negative definite on $V$. From $C_{c}^{\infty}(\Omega) \leq \mathcal{D}\left(L_{M}\right)$, $\operatorname{ind}_{L}(M) \geq n$ and this shows that $\operatorname{ind}_{L}(M) \geq \widetilde{\operatorname{ind}_{L}}(M)$. To prove the reversed inequality, we shall need the property that $C_{c}^{\infty}(M)$ is a core for the Friedrichs extension $L_{M}$. Indeed, in this case $\mathcal{D}(L)$ can be replaced with $C_{c}^{\infty}(M)$ and the proof follows the same lines as before. For each finite dimensional $V \leq C_{c}^{\infty}(M)$ where $Q$ is negative definite, up to taking $\Omega$ sufficiently large to contain the support of a basis of $V$ we obtain $V \leq C_{c}^{\infty}(\Omega)$. Using the definitions, $\operatorname{ind}_{L}(M) \leq \sup _{\Omega \Subset M} \operatorname{ind}_{L}(\Omega)=$ $\operatorname{ind}_{L}(M)$.

The next theorem is a celebrated result of W. Allegretto, D. Fischer-Colbrie, R. Gulliver and I.M. Glazman, see also [PRS08], Lemma 3.16. We stress that, as for the above results, the proof for $L=-\Delta-q(x)$ can be repeated, almost word-by-word, for general $L$. 
Theorem 1.40 ([All74], [FC85], [Gul88], [Gla65] pp.158-159). Let $(M,\langle\rangle$, be a Riemannian manifold, and assume that $\operatorname{ind}_{L}(M)<+\infty$. Then, there exists an open, relatively compact set $\Omega$ such that $\lambda_{1}^{L}(M \backslash \bar{\Omega}) \geq 0$, that is, the Friedrichs extension $L_{M \backslash \bar{\Omega}}$ is non-negative.

Proof. The proof is substantially that of [FC85], up to removing the completeness assumption. Let $\left\{\Omega_{j}\right\} \uparrow M$ be a smooth exhaustion of $M$ by means of open, relatively compact smooth domains. If $\lambda_{1}^{L}\left(\Omega_{j}\right) \geq 0$ for every $j$, we are done by setting $\Omega=\emptyset$, otherwise there exists some $j$ such that $\lambda_{1}^{L}\left(\Omega_{j}\right)<0$. Without loss of generality, we can assume $j=1$. Since $\left(L, C_{c}^{\infty}\left(\Omega_{1}\right)\right)$ is essentially self-adjoint, we can choose $\phi_{1} \in C_{c}^{\infty}\left(\Omega_{1}\right)$ such that $\left(L \phi_{1}, \phi_{1}\right)_{L^{2}}<0$. Now we consider the annuli $A_{j}=\Omega_{j} \backslash \bar{\Omega}_{1}$. If, for every $j, \lambda_{1}^{L}\left(A_{j}\right) \geq 0$ we are done by setting $\Omega=\Omega_{1}$, otherwise there exists $j$ (say $j=2$ ) such that $\lambda_{1}^{L}\left(A_{j}\right)<0$. We choose $\phi_{2} \in C_{c}^{\infty}\left(A_{2}\right)$ such that $\left(L \phi_{2}, \phi_{2}\right)_{L^{2}}<0$, and we note that the supports of $\phi_{1}$ and $\phi_{2}$ are disjoint subsets of $\Omega_{2}$ since $\Omega_{1}$ and $A_{2}$ are. Repeating the argument on the annuli $\Omega_{j} \backslash \bar{\Omega}_{2}$ and so on, we can find linearly independent functions $\left\{\phi_{i}\right\}$ that make the Rayleigh quotient negative. Since $\widetilde{\operatorname{ind}}_{L}(M)<+\infty$, there are only finitely many $\phi_{i}$, say $n$, hence $\lambda_{1}^{L}\left(\Omega_{j} \backslash \bar{\Omega}_{n}\right) \geq 0$ for every $j>n$. Letting $j \rightarrow+\infty$ we deduce the claim.

The problem whether stability outside some compact set $\Omega$, that is,

$$
\lambda_{1}^{L}(M \backslash \bar{\Omega}) \geq 0
$$

is equivalent to $\operatorname{ind}_{L}(M)<+\infty$ has been affirmatively solved, in Euclidean setting, by J. Piepenbrink in $[\mathbf{P i e 7 4 b}],[\mathbf{P i e} 74 \mathbf{a}],[\mathbf{P i e} 7 \mathbf{7}]$ under the growth requirement (1.81). Recently, the result has been given a new, different proof by B. Devyver, [Dev] for complete manifolds and for operators with $A=\mathrm{Id}$. The interested reader should also consult [RS78], Chapter 15 and Theorem RSK in [AK]. Here, according to $[\mathbf{M a r}]$, we present a generalization of Piepenbrink and Devyver's results. Next theorem shall also be compared with Proposition 2 of [FC85].

Theorem 1.41. Let $(M,\langle\rangle$,$) be a Riemannian manifold, and let \left(L, C_{c}^{\infty}(M)\right)$ be as in (1.66). Then, the following statements are equivalent:

(i) $L$ is bounded from below on $C_{c}^{\infty}(M)$ and there exists a relatively compact set $\Omega$ such that $L$ is stable on $M \backslash \bar{\Omega}$;

(ii) There exists a $C^{1}$ function $w>0$, defined outside some relatively compact set $\Omega$, such that $L w=0$ weakly on $M \backslash \bar{\Omega}$;

(iii) There exists a $H_{\mathrm{loc}}^{1}$ function $w \geq 0, w \neq \equiv 0$, defined a.e. outside some relatively compact set $\Omega$, such that $L w \geq 0$ weakly on $M \backslash \bar{\Omega}$;

(iv) $\widetilde{\operatorname{ind}}_{L}(M)<+\infty$;

(v) $L$ is bounded from below on $C_{c}^{\infty}(M)$ and its Friedrichs extension $L_{M}$ satisfies $\operatorname{ind}_{L}(M)<+\infty$, dim $\operatorname{ker}\left(L_{M}\right)<+\infty$;

Moreover, if any of the conditions holds, $\operatorname{ind}_{L}(M)=\widetilde{\operatorname{ind}_{L}}(M)$.

Proof. $(v) \Rightarrow(i v)$. If $(v)$ holds, by Proposition $1.39 \widetilde{\operatorname{ind}}_{L}(M)=\operatorname{ind}_{L}(M)<$ $+\infty$, and this proves both (iv) and the last statement.

$(i) \Leftrightarrow(i i) \Leftrightarrow($ iii $)$ is Theorem 1.33, together with Remark 1.35 and Corollary 1.37. $(i v) \Rightarrow(i)$. By Theorem $1.40, L$ is stable outside some $\Omega \Subset M$, and thus by Proposition $1.37 L$ is bounded from below on $C_{c}^{\infty}(M)$, as required. 
$(i) \Rightarrow(v)$. We basically follow the ideas in [Pie74a], Theorem 4.1. If $(i)$ holds, let $w \in C^{1}(M \backslash \Omega)$ be the weak solution of $L w=0$ given by $(i) \Leftrightarrow(i i)$, that is,

$$
\int_{M} A(\nabla w, \nabla \phi)=\int_{M} q w \phi \quad \text { for every } \phi \in \operatorname{Lip}_{c}(M \backslash \bar{\Omega}),
$$

where $\operatorname{Lip}_{c}(M \backslash \bar{\Omega})$ can be substituted by its closure in the norm induced by the quadratic form $Q$ in (1.82) restricted to $C_{c}^{\infty}(M \backslash \bar{\Omega}) \times C_{c}^{\infty}(M \backslash \bar{\Omega})$. In particular, (1.90) holds for any $\phi \in \mathcal{D}\left(L_{M \backslash \bar{\Omega}}\right)$. Fix a relatively compact domain with smooth boundary $\Omega^{\prime}$ such that $\Omega \Subset \Omega^{\prime}$, and let $\varepsilon<\operatorname{dist}\left(\Omega, M \backslash \Omega^{\prime}\right) / 2$ be sufficiently small that the function $\operatorname{dist}\left(\cdot, M \backslash \Omega^{\prime}\right)$ is smooth on $B_{\varepsilon}\left(M \backslash \Omega^{\prime}\right)$. Denote with $\nu$ the outward pointing unit normal to $\partial \Omega^{\prime}$. Consider the Lipschitz functions

$$
\varphi_{\varepsilon}(x)= \begin{cases}0 & \text { if } \operatorname{dist}\left(x, M \backslash \Omega^{\prime}\right) \geq \varepsilon ; \\ \frac{1}{\varepsilon}\left[\varepsilon-\operatorname{dist}\left(x, M \backslash \Omega^{\prime}\right)\right] & \text { if } \operatorname{dist}\left(x, M \backslash \Omega^{\prime}\right) \in(0, \varepsilon) \\ 1 & \text { if } x \in \Omega^{\prime}\end{cases}
$$

and an arbitrary $\eta \in \mathcal{D}\left(L_{M}\right)$. We recall that, by Remark 1.32, $\eta \in H_{\mathrm{loc}}^{2}(M)$. Then, applying (1.90) to the test function $\phi=\eta \varphi_{\varepsilon} \in \mathcal{D}\left(L_{M \backslash \bar{\Omega}}\right)$, letting $\varepsilon \rightarrow 0$ and using the coarea formula ([EG92], Theorem 2 p.117) we deduce

$$
\int_{M \backslash \Omega^{\prime}} A(\nabla w, \nabla \eta)+\int_{\partial \Omega^{\prime}} A(\nabla w, \nu) \eta \mathrm{d} \sigma=\int_{M \backslash \Omega^{\prime}} q w \eta, \quad \forall \eta \in \mathcal{D}\left(L_{M}\right),
$$

where, in the integral on $\partial \Omega^{\prime}, \mathrm{d} \sigma$ is the $(m-1)$-dimensional Hausdorff measure, and the integrand is meant to be in the trace sense. Up to renaming, we write $\Omega$ instead of $\Omega^{\prime}$. Fix $u \in \mathcal{D}\left(L_{M}\right)$, and apply (1.91) to $\eta=u^{2} / w$ to obtain

$$
2 \int_{M \backslash \Omega} \frac{u}{w} A(\nabla w, \nabla u)-\int_{M \backslash \Omega} \frac{u^{2}}{w^{2}} A(\nabla w, \nabla w)+\int_{\partial \Omega} A(\nabla w, \nu) \frac{u^{2}}{w} \mathrm{~d} \sigma=\int_{M \backslash \Omega} q u^{2} .
$$

From

$$
0 \leq A\left(\nabla\left(\frac{u}{w}\right), \nabla\left(\frac{u}{w}\right)\right)=\frac{1}{w^{2}} A(\nabla u, \nabla u)+\frac{u^{2}}{w^{4}} A(\nabla w, \nabla w)-2 \frac{u}{w^{3}} A(\nabla w, \nabla u),
$$

Multiplying the last equality by $w^{2}$, integrating and inserting into (1.92) we get

$$
\int_{M \backslash \Omega}\left[A(\nabla u, \nabla u)-q u^{2}\right] \geq-\int_{\partial \Omega} \frac{u^{2}}{w} A(\nabla w, \nu) \mathrm{d} \sigma .
$$

Next, we consider $u$ on $\Omega$. Let $Q$ be the following quadratic form:

$$
Q(\phi, \phi)=\int_{\Omega}\left[A(\nabla \phi, \nabla \phi)-q \phi^{2}\right]-\int_{\partial \Omega} \frac{1}{w} A(\nabla w, \nu) \phi^{2} \mathrm{~d} \sigma \quad \forall \phi \in C^{\infty}(\bar{\Omega}) .
$$

Since $q, A \in L^{\infty}(\Omega)$ and $w \in C^{1}(\partial \Omega), Q$ is bounded from below on $C^{\infty}(\bar{\Omega})$ and its closure is on $H^{1}(\Omega) \times H^{1}(\Omega)$. By elliptic regularity up to the boundary, the solution $u$ of the Euler-Lagrange equations

$$
0=Q(u, \phi)=\int_{\Omega}[A(\nabla u, \nabla \phi)-q u \phi]-\int_{\partial \Omega} \frac{1}{w} A(\nabla w, \nu) u \phi \mathrm{d} \sigma \quad \forall \phi \in H^{1}(\Omega) .
$$


is in $H^{2}(\Omega)$. Integrating by parts, $u$ solves

$$
\left\{\begin{array}{l}
L u=0 \quad \text { on } \Omega, \\
A(\nabla u, \nu)-[A(\nabla \log w, \nu)] u=0 .
\end{array}\right.
$$

Let $\left\{\sigma_{k}\right\}$ be the set of min-max levels of the self-adjoint extension of $\left(L, C^{\infty}(\bar{\Omega})\right)$ associated to the closure of $Q$. We claim that $\sigma_{k} \rightarrow+\infty$, so that there is no essential spectrum. This follows from a standard compactness argument that we now recall. Since $A$ is uniformly elliptic on $\Omega$, there exists a constant $c>0$ such that

$$
|Q(\phi, \phi)| \geq c\|\nabla \phi\|_{L^{2}(\Omega)}^{2}-\|q\|_{L^{\infty}(\Omega)}\|\phi\|_{L^{2}(\Omega)}^{2}-\|A(\nabla \log w, \nu)\|_{L^{\infty}(\partial \Omega)}\|\phi\|_{L^{2}(\partial \Omega)}^{2} .
$$

The trace theorem ([EG92], p.134) and Young inequality imply that, for some positive constants $\widetilde{C}, C$ with $C=C(\varepsilon)$,

$$
\|\phi\|_{L^{2}(\partial \Omega)}^{2} \leq \int_{\Omega}\left|\nabla \phi\|\phi \mid+\widetilde{C}\| \phi\left\|_{L^{2}(\Omega)}^{2} \leq \varepsilon\right\| \nabla \phi\left\|_{L^{2}(\Omega)}^{2}+C\right\| \phi \|_{L^{2}(\Omega)}^{2} .\right.
$$

Inserting into the above inequality and choosing $\varepsilon$ sufficiently small, we deduce that, for some constant $C>0$,

$$
|Q(\phi, \phi)| \geq \frac{c}{2}\|\nabla \phi\|_{L^{2}(\Omega)}^{2}-C\|\phi\|_{L^{2}(\Omega)}^{2} \quad \forall \phi \in H^{1}(\Omega) .
$$

Assume by contradiction that $\sigma_{k} \rightarrow \sigma$, for some $\sigma \in \mathbb{R}$, and let $\left\{v_{k}\right\} \subset H^{2}(\Omega)$ be the associated set of orthogonal eigenfunctions, normalized in $L^{2}$-norm. Then by (1.95) $\left\{v_{k}\right\}$ should be bounded in $H^{1}$ norm, and by Rellich-Kondrachov theorem some subsequence of $\left\{v_{k}\right\}$ should converges in $L^{2}(\Omega)$, which contradicts the orthonormality. Therefore, we can consider the greatest index $k_{1}$ such that $\sigma_{k_{1}}<0$, and the greatest index $k_{2} \geq k_{1}$ such that $\sigma_{k_{2}}=0$. Define

$$
V_{-}=<v_{1}, \ldots, v_{k_{1}}>\subset L^{2}(\Omega), \quad V_{0}=<v_{k_{1}+1}, \ldots, v_{k_{2}}>\subset L^{2}(\Omega)
$$

and $V=V_{-}+V_{0}$. Note that these subspaces are contained in $H^{2}(\Omega)$. By the min-max characterization, for every nonzero $u \in H^{2}(\Omega), u \perp V$ we deduce

$$
0<\sigma_{k_{2}+1}\|u\|_{L^{2}(\Omega)}^{2} \leq Q(u, u)=\int_{\Omega}\left[A(\nabla u, \nabla u)-q u^{2}\right]-\int_{\partial \Omega} \frac{1}{w} A(\nabla w, \nu) u^{2} \mathrm{~d} \sigma .
$$

Extending each $v_{k}$ to be zero outside $\Omega$, and summing up (1.94) and (1.96) we obtain

$$
\left(L_{M} u, u\right)_{L^{2}}=\int_{M}\left[A(\nabla u, \nabla u)-q u^{2}\right] \begin{cases}\geq 0 & \forall u \in \mathcal{D}\left(L_{M}\right) \cap V_{-}^{\perp}, \\ \geq \sigma_{k_{2}+1}\|u\|_{L^{2}(\Omega)}^{2} & \forall u \in \mathcal{D}\left(L_{M}\right) \cap V^{\perp} .\end{cases}
$$

It is now easy to deduce that

$$
\text { (1) } \operatorname{ind}\left(L_{M}\right) \leq k_{1}, \quad \text { (2) } \quad \operatorname{dim} \operatorname{ker}\left(L_{M}\right) \leq k_{2} .
$$

For the ease of completeness we give a detailed proof of it. For each given $r$ dimensional subspace $F \leq \mathcal{D}\left(L_{M}\right)$, denote with $\left\{f_{i}\right\}$ a chosen basis for $F$, and with $F_{\mid \Omega}$ the subspace of $L^{2}(\Omega)$ obtained by restricting each function $f \in F$ to $\Omega$. We first address inequality (1). Suppose thus that $Q$ is negative definite on $F$. We claim that $\left\{f_{i}\right\}$ is still a basis when restricted to $\Omega$. Indeed, assume by contradiction that 
$f=\lambda^{i} f_{i}$ is a non-zero linear combination which is identically zero on $\Omega$. Then, since the trace of $f$ on $\partial \Omega$ is zero, by (1.94)

$$
\left(L_{M} f, f\right)_{L^{2}}=Q(f, f) \equiv \int_{M \backslash \Omega}\left[A(\nabla f, \nabla f)-q f^{2}\right] \geq 0,
$$

which contradicts the fact that $Q$ is negative definite on $F$. To prove (1), we show that $r \leq k_{1}$. Suppose by contradiction that $r>k_{1}$. Then, since $F_{\mid \Omega}$ is still $r$ dimensional, there exists a nonzero $f \in F_{\mid \Omega} \cap V_{-}^{\perp}$. Writing $f=\lambda^{i} f_{i}, f \in \mathcal{D}\left(L_{M}\right)$ and, by (1.97), $\left(L_{M} f, f\right)_{L^{2}} \geq 0$, contradicting the fact that $Q$ is negative definite on $F$.

In a similar way, we prove (2). Let thus $F$ be an $r$-dimensional subspace of $\operatorname{ker}\left(L_{M}\right)$. We first show that $F_{\mid \Omega}$ is still $r$-dimensional by verifying that $\left\{f_{i}\right\}$ restricted to $\Omega$ is still a basis. Indeed, if this is not so, let $f=\lambda^{i} f_{i}$ is a non-zero linear combination which is identically zero on $\Omega$. Again by (1.94) and since $f \in \operatorname{ker}\left(L_{M}\right)$ we deduce

$$
0=\left(L_{M} f, f\right)_{L^{2}}=Q(f, f) \equiv \int_{M \backslash \Omega}\left[A(\nabla f, \nabla f)-q f^{2}\right] \geq 0 .
$$

Therefore, inequality in (1.94) must be an equality. In particular, (1.93) must be an equality, and being $A$ positive definite necessarily $f=C w$ for some positive constant $C$. This is a contradiction since the trace of $f$ on $\partial \Omega$ is zero while $w$ is positive and continuous on $\partial \Omega$. Next, suppose by contradiction that $r>k_{2}$. Then, since $F_{\mid \Omega}$ is still $r$-dimensional, there exists a nonzero $f \in F_{\mid \Omega} \cap V^{\perp}$. Writing $f=\lambda^{i} f_{i}, f \in \operatorname{ker}\left(L_{M}\right)$ and, by (1.97),

$$
0=\left(L_{M} f, f\right)_{L^{2}} \geq \sigma_{k_{2}+1}\|f\|_{L^{2}(\Omega)}^{2},
$$

which implies that $f$ is identically zero on $\Omega$. Now, we proceed as above to show that $f=C w$ for some positive constant $C$, which gives the desired contradiction.

REMARK 1.42. The above theorem has the immediate consequence that the finiteness of $\operatorname{ind}_{L}(M)$ is a stable property under compactly supported variations of the potential $q(x)$. 


\section{CHAPTER 2}

\section{Some geometric examples related to oscillation theory}

The purpose of this section is to describe some geometric problems where the study of the oscillations of a suitable ODE has an important role. In Section 2.1, we discuss an ODE approach to compactness results for Riemannian manifolds in the spirit of the classical Bonnet-Myers theorem [Mye41]. Then, in Section 2.2 we show how very similar techniques can be used to get information on the spectrum of the Laplacian on a complete, non-compact manifold $M$. In fact, we can even obtain spectral estimates by analyzing smooth maps, in particular isometric immersions, from $M$ to some manifold $N$, and in Section 2.3 we discuss a prototype example. As observed in the Introduction, the ODE approach has important applications in the spectral theory of Schrödinger operators. In turn, spectral assumptions are often used to obtain existence or non-existence of solutions of semilinear PDE. In this respect, the Yamabe problem is very well suited to describe this interplay, that we analyze in Section 2.4 below.

\subsection{Conjugate points and Myers type compactness results}

With the appearance of the classical Bonnet-Myers theorem, [Mye41], on the compactness of a complete manifold under an appropriate Ricci curvature condition, an entire field of research rose to clarify the interplay between curvature, Jacobi fields and conjugate points. This relationship has been investigated by many authors, notably E. Calabi [Cal67], J. Cheeger, M. Gromov and M. Taylor [CGT82] and, more recently, for instance by J.H. Eschenburg and J.J. O'Sullivan [EO80], G. Galloway [Gal82] and D. Kupeli [Kup86]. In particular, these latter have shown that the original Bonnet-Myers problem can be shifted to the analysis of the solutions $g$ of the ODE $g^{\prime \prime}-G g=0$, for a suitable function $G$ related to geometry. On the other hand, the above ODE has been the subject of an intensive independent research in the last century (for an account, see [Swa68], [Har64b]), and the possibility of exploiting these available analytical results has highly improved the original conclusions of Bonnet and Myers. To briefly explain this approach, we begin with deriving Myers theorem from the Laplacian comparison Theorem 1.19. Let $M$ be complete and assume that

$$
\operatorname{Ricc}(\nabla r, \nabla r) \geq-(m-1) G(r) .
$$

Then, by (1.44) the domain $D_{o}$ of the normal coordinates is a subset of $B_{R_{0}}$, where $R_{0} \leq+\infty$ is the first zero of any solution $g$ of

$$
\left\{\begin{array}{l}
g^{\prime \prime}-G g \geq 0, \\
g(0)=0, \quad g^{\prime}(0)=1 .
\end{array}\right.
$$


If $R_{0}<+\infty$, since $\bar{D}_{o}=M$ we deduce that $M$ is bounded. Since $M$ is complete, it is compact and the diameter of $M$ does not exceed $2 R_{0}$. This is the case, for instance, when $G(r)=-B^{2}$ for some $B>0$, that is,

$$
\operatorname{Ricc}(\nabla r, \nabla r)(x) \geq(m-1) B^{2},
$$

for which we can choose $g(r)=B^{-1} \sin (B r)$ and $M$ is compact with diameter at most $2 \pi / B$. Therefore, if

$$
\operatorname{Ricc} \geq(m-1) B^{2}\langle,\rangle
$$

we recover the classical Bonnet-Myers theorem [Mye41]. The improvement from $\operatorname{diam}(M) \leq 2 \pi / B$ to the sharp $\operatorname{diam}(M) \leq \pi / B$ comes from the fact that $(2.3)$ is independent of the point $o$. The above discussion shows, following the way outlined by Galloway in [Gal82], that we can prove compactness of $M$ via Theorem 1.19 without making use of Morse index techniques. There is, however, a technical unpleasant restriction in the approach we have just described, that is, the bound $G(r)$ is independent of the geodesics emanating from $o$. For this reason, we pause to reproduce the reasoning in $[\mathbf{G a l} 82]$.

ThEOREM 2.1 ([Gal82], Lemma 1). Let $(M,\langle\rangle$,$) be a complete Riemannian$ manifold of dimension $m \geq 2$. Assume that, for some origin o and for every unit speed geodesic $\gamma: \mathbb{R}_{0}^{+} \rightarrow M$ emanating from $o$, the solution $g$ of

$$
\left\{\begin{array}{l}
g^{\prime \prime}+\frac{\operatorname{Ricc}\left(\gamma^{\prime}, \gamma^{\prime}\right)(s)}{m-1} g=0, \\
g(0)=0, \quad g^{\prime}(0)=1
\end{array}\right.
$$

has a first zero. Then, $M$ is compact with finite fundamental group.

The main step of the proof is the following well known lemma. We report the nice argument in [PRRS11] that avoids the use of variational arguments.

LEMma 2.2 ([PRRS11], Lemma 8.2). Let $(M,\langle\rangle$,$) be a complete Riemannian$ manifold of dimension $m \geq 2$, and let $\gamma:\left[0, r_{0}\right] \rightarrow M$ be a unit speed geodesic starting from $o$ and ending at $q=\gamma\left(r_{0}\right)$. If $\gamma$ is length minimizing on $\left[0, r_{0}\right]$, then for every $h \in \operatorname{Lip}\left(\left[0, r_{0}\right]\right)$ such that $h(0)=h\left(r_{0}\right)=0$ we have

$$
\int_{0}^{r_{0}}\left(h^{\prime}(s)\right)^{2} \mathrm{~d} s-\int_{0}^{r_{0}}\left(\frac{\operatorname{Ricc}\left(\gamma^{\prime}, \gamma^{\prime}\right)(s)}{m-1}\right) h^{2}(s) \mathrm{d} s \geq 0
$$

Proof. First, assume that $q$ is not the cut-point for $o$ along $\gamma$, so that the distance function $r$ is smooth. Using Newton inequality $|\operatorname{Hess}(r)|^{2} \geq(\Delta r)^{2} /(m-1)$ in (1.46) we deduce that, along $\gamma$,

$$
(\Delta r)^{\prime}+\frac{(\Delta r)^{2}}{m-1}+\operatorname{Ricc}\left(\gamma^{\prime}, \gamma^{\prime}\right) \leq 0
$$

Fix $0<\varepsilon<r_{0}$. Multiplying by $h^{2}$ and integrating on $\left(\varepsilon, r_{0}\right]$ we get

$$
\int_{\varepsilon}^{r_{0}} h^{2}(\Delta r)^{\prime} \mathrm{d} s+\int_{\varepsilon}^{r_{0}}\left(\frac{h^{2}(\Delta r)^{2}}{m-1}+\operatorname{Ricc}\left(\gamma^{\prime}, \gamma^{\prime}\right) h^{2}\right) \mathrm{d} s \leq 0 .
$$

By the asymptotic behaviour of $\Delta r$ in $(1.47), h^{2}(\varepsilon)(\Delta r)(\varepsilon) \rightarrow 0$ as $\varepsilon \rightarrow 0^{+}$. Hence, integrating by parts the first term and letting $\varepsilon \rightarrow 0^{+}$we deduce

$$
\int_{0}^{r_{0}}\left(\frac{h^{2}(\Delta r)^{2}}{m-1}+\operatorname{Ricc}\left(\gamma^{\prime}, \gamma^{\prime}\right) h^{2}\right) \mathrm{d} s \leq \int_{0}^{r_{0}} 2 h h^{\prime}(\Delta r) \mathrm{d} s .
$$


By Young inequality, the integrand on the RHS can be rearranged as follows:

$$
2 h h^{\prime}(\Delta r) \leq \frac{h^{2}(\Delta r)^{2}}{m-1}+(m-1)\left(h^{\prime}\right)^{2},
$$

and inserting into (2.7) we obtain (2.5). Now, assume that $q$ is a cut-point for $o$ along $\gamma$, and use Calabi trick. Let $\varepsilon>0$ be small and define

$$
o_{\varepsilon}=\gamma(\varepsilon), \quad r_{\varepsilon}(x)=d\left(x, o_{\varepsilon}\right), \quad \gamma_{\varepsilon}:\left[0, r_{0}-\varepsilon\right] \rightarrow M, \quad \gamma_{\varepsilon}(s)=\gamma(\varepsilon+s) .
$$

Then, $q$ is not a cut-point of $o_{\varepsilon}$ along $\gamma_{\varepsilon}$, so that (2.6) holds for $r_{\varepsilon}, \gamma_{\varepsilon}$. Consider a Lipschitz function $h$ with compact support in $\left(0, r_{0}\right)$, and set $h_{\varepsilon}(r)=h(r+\varepsilon)$. We choose $\varepsilon$ to be sufficiently small that $h_{\varepsilon}(0)=0$. Multiply (2.6) for $h_{\varepsilon}^{2}$ and integrate on $\left[\delta, r_{0}-\varepsilon\right]$, for some small $\delta>0$ to deduce

$$
\int_{\delta}^{r_{0}-\varepsilon} h_{\varepsilon}^{2}\left(\Delta r_{\varepsilon}\right)^{\prime} \mathrm{d} s+\int_{\delta}^{r_{0}-\varepsilon}\left(\frac{h_{\varepsilon}^{2}\left(\Delta r_{\varepsilon}\right)^{2}}{m-1}+\operatorname{Ricc}\left(\gamma_{\varepsilon}^{\prime}, \gamma_{\varepsilon}^{\prime}\right) h_{\varepsilon}^{2}\right) \mathrm{d} s \leq 0
$$

By the asymptotic behaviour of $\Delta r_{\varepsilon}$ near $r_{\varepsilon}=0$, and since $h_{\varepsilon}(0)=0$, we can integrate by parts and let $\delta \rightarrow 0$ as above to get

$$
\begin{aligned}
& \int_{0}^{r_{0}-\varepsilon}\left(\frac{h_{\varepsilon}^{2}\left(\Delta r_{\varepsilon}\right)^{2}}{m-1}+\operatorname{Ricc}\left(\gamma_{\varepsilon}^{\prime}, \gamma_{\varepsilon}^{\prime}\right) h_{\varepsilon}^{2}\right) \mathrm{d} s \leq \int_{0}^{r_{0}-\varepsilon} 2 h_{\varepsilon} h_{\varepsilon}^{\prime}\left(\Delta r_{\varepsilon}\right) \mathrm{d} s \\
& \leq \int_{0}^{r_{0}-\varepsilon}\left(\frac{h_{\varepsilon}^{2}\left(\Delta r_{\varepsilon}\right)^{2}}{m-1}+(m-1)\left(h_{\varepsilon}^{\prime}\right)^{2}\right) \mathrm{d} s,
\end{aligned}
$$

hence

$$
\int_{0}^{r_{0}-\varepsilon}\left(h_{\varepsilon}^{\prime}(s)\right)^{2} \mathrm{~d} s-\int_{0}^{r_{0}-\varepsilon}\left(\frac{\operatorname{Ricc}\left(\gamma_{\varepsilon}^{\prime}, \gamma_{\varepsilon}^{\prime}\right)(s)}{m-1}\right) h_{\varepsilon}^{2}(s) \mathrm{d} s \geq 0 .
$$

It is enough to change variables to recover (2.5) for every Lipschitz $h$ with compact support in $\left(0, r_{0}\right)$. A density argument gives $(2.5)$ for every $h \in \operatorname{Lip}\left(\left[0, r_{0}\right]\right)$ with zero boundary conditions.

REMARK 2.3. The above proof basically reflects the 1-dimensional case of the implication (ii) $\Rightarrow$ (iii) in Theorem 1.33 (see the proof of Theorem 1 of [FCS80]). Indeed, (2.5) is equivalent to say

$$
\lambda_{1}^{L}\left(\left[0, r_{0}\right]\right) \geq 0, \quad \text { where } \quad L=-\frac{\mathrm{d}^{2}}{\mathrm{~d} s^{2}}-\frac{\operatorname{Ricc}\left(\gamma^{\prime}, \gamma^{\prime}\right)(s)}{m-1} .
$$

On the other hand, if $\Delta r$ satisfies (2.6) on some segment $\gamma_{\left[\left[0, r_{0}\right]\right.}$, by (1.15) the function

$$
u(s)=s \exp \left\{\int_{0}^{s}\left(\frac{\Delta r \circ \gamma(\sigma)}{m-1}-\frac{1}{\sigma}\right) \mathrm{d} \sigma\right\}
$$

is well defined, positive on $\left(0, r_{0}\right)$ and solves $L u \geq 0$, see also Proposition 1.13.

Proof of Theorem 2.1. If (2.4) admits a (smooth) solution $g$ such that $g$ has a first zero at some $r_{0}>0$, then $g$ solves

$$
0=(L g, g)_{L^{2}}=\int_{0}^{r_{0}}\left(g^{\prime}(s)\right)^{2} \mathrm{~d} s-\int_{0}^{r_{0}}\left(\frac{\operatorname{Ricc}\left(\gamma^{\prime}, \gamma^{\prime}\right)(s)}{m-1}\right) g^{2}(s) \mathrm{d} s,
$$

where $L$ is as in (2.8). Therefore, by the min-max principle $\lambda_{1}^{L}\left(\left[0, r_{0}\right]\right) \leq 0$, and by monotonicity of eigenvalues $\lambda_{1}^{L}\left(\left[0, r_{1}\right]\right)<0$ for every $r_{1}>0$. The above lemma implies that $\gamma$ is not length minimizing after $r_{0}$, so that there exists a cut-point (in fact, a conjugate point) of $o$ along $\gamma$. Since this happens for every $\gamma, M$ is compact by Theorem 1.1. The argument above can be repeated verbatim for the 
Riemannian universal covering $\widetilde{M} \rightarrow M$ to show that $\widetilde{M}$ is compact. Hence, the fundamental group of $M$ is finite.

The "converse" of the above statement comes from an application of the matrix Riccati comparison.

Proposition 2.4. Let $(M,\langle\rangle$,$) be a Riemannian manifold of dimension m \geq$ 2 , and let $\gamma: \mathbb{R}_{0}^{+} \rightarrow M$ be a unit speed geodesic emanating from some origin $o$. Denote with $K_{\mathrm{rad}}(s)$ the radial sectional curvature at $\gamma(s)$, and assume that

$$
K_{\text {rad }}(s) \leq-G(s) .
$$

If the solution $g$ of

$$
\left\{\begin{array}{l}
g^{\prime \prime}-G g=0 \\
g(0)=0, \quad g^{\prime}(0)=1
\end{array}\right.
$$

is positive on $\mathbb{R}^{+}$, then there is no conjugate point to o along $\gamma$. If $M$ is complete and this happens for every $\gamma$ emanating from o, then o has no conjugate points and $\exp _{o}$ is a covering map.

Proof. Let $\left(0, s_{1}\right)$ be the maximal interval such that $o$ is free of conjugate points on $\left(0, s_{1}\right)$. Assume by contradiction that $s_{1}<+\infty$. By the discussion in Section 1.2, the Jacobi tensor $J$ along $\gamma$ has nontrivial kernel at $s_{1}$, and the function $B=J^{\prime} J^{-1}$ is unbounded from below as $s \rightarrow s_{1}^{-}$. Note that $B$ solves, in a parallel orthonormal frame along $\gamma$,

$$
\left\{\begin{array}{l}
B^{\prime}+B^{2}+R_{\gamma}=0 \quad \text { on }\left(0, s_{1}\right) \\
B(s)=s^{-1} I+o(1) \quad \text { as } s \rightarrow 0^{+} .
\end{array}\right.
$$

and $R_{\gamma}$ is defined as in (1.30). By (2.9) and (1.32), $R_{\gamma} \leq-G(s) I$. Setting, in a parallel orthonormal frame along $\gamma, B_{g}=\left(g^{\prime} / g\right) I, B_{g}$ solves

$$
\left\{\begin{array}{l}
B_{g}^{\prime}+B_{g}^{2}=G I \leq-R_{\gamma} \quad \text { on } \mathbb{R}^{+} \\
B_{g}(s)=s^{-1} I+o(1) \quad \text { as } \quad s \rightarrow 0 .
\end{array}\right.
$$

By the matrix Riccati Comparison 1.14, $B \geq B_{g}$ as a quadratic form. Hence, since $B_{g}$ is defined on the whole $\mathbb{R}^{+}, B$ cannot be unbounded from below as $s \rightarrow s_{0}^{-}$, contradiction.

REMARK 2.5. The above proposition can indeed be proved as a direct application of the Rauch comparison theorem ([Car92], p. 215). It should be observed that Rauch theorem, however, is not a straightforward consequence of the sole matrix Riccati comparison, but it also requires the Index lemma ([Car92], p.212).

Next corollary follows from Proposition 1.21.

Corollary 2.6. Let $(M,\langle\rangle$,$) be a complete, connected Riemannian manifold$ of dimension $m \geq 2$. Assume that, along some unit speed geodesic $\gamma: \mathbb{R}_{0}^{+} \rightarrow M$, the radial sectional curvature $K_{\gamma}(s)$ satisfy

$$
\left(K_{\gamma}\right)_{+} \in L^{1}\left(\mathbb{R}^{+}\right), \quad s \int_{s}^{+\infty}\left(K_{\gamma}\right)_{+}(s) \leq \frac{1}{4}
$$

Then, there exists no conjugate point to $\gamma(0)$ along $\gamma$. Moreover, if (2.12) holds for every ray emanating from some $o \in M$, then $o$ has no conjugate points and $\exp _{o}: T_{o} M \rightarrow M$ is a covering map. 
The existence of a first zero of a solution $g$ of either (2.1) or (2.4) can be guaranteed, for instance, by classical oscillation results. Among the various criteria, that of Hille-Nehari is one of the finest, see [Swa68], p.45. For the convenience of the reader, we recall here this result in the simple form given by E. Hille, [Hil48].

Theorem 2.7 ([Swa68], p.45 and [Hil48], Theorem 5 and Corollary 1). Let $K \in C^{0}(\mathbb{R}) \cap L^{1}(+\infty)$ be non-negative, and consider the ODE $g^{\prime \prime}+K g=0$. Denote with $k(s), k_{*}$ and $k^{*}$ respectively the quantities

$$
k(s)=s \int_{s}^{+\infty} K(\sigma) \mathrm{d} \sigma, \quad k_{*}=\liminf _{s \rightarrow+\infty} k(s), \quad k^{*}=\limsup _{s \rightarrow+\infty} k(s) .
$$

Then,

- If the ODE is nonoscillatory, then necessarily $k_{*} \leq \frac{1}{4}$ and $k^{*} \leq 1$.

- If $k(s) \leq \frac{1}{4}$ for $s$ large enough, in particular if $k^{*}<1 / 4$, then the ODE is nonoscillatory.

As a consequence, $k_{*}>\frac{1}{4}$ is a sufficient condition for the equation to be oscillatory.

REMARK 2.8. If $K \notin L^{1}(+\infty)$, the result applies with $k_{*}=k^{*}=+\infty$, and the equation $g^{\prime \prime}+K g=0$ is oscillatory. This case is due to W.B. Fite [Fit18]. Note the strict analogy with condition (1.52) for the positivity of $g$, although the techniques used in [Hil48] to prove Theorem 2.7 are different from those of Proposition 1.21.

There are two main questions that, at the best of our knowledge, are still almost unanswered. The first regards the search of conditions in finite form for the existence of a first zero, that is, conditions involving the potential $K$ only in a compact interval. The second is how to deal with possibly negative $K$. In this last direction, the first instance of a result that allows $K$ to change sign is due to W. Ambrose [Amb57] and A. Wintner [Win49] (consult also [Gui92], Corollaries 3.5 and 3.6 for a different proof and a generalization). This was extended by R. Moore [Moo55] to the following

Theorem 2.9 ([Moo55], Theorem 2). Let $K \in C^{0}(\mathbb{R})$. Equation $g^{\prime \prime}+K g=0$ is oscillatory provided that, for some $\lambda \in[0,1)$, there exists

$$
\lim _{s \rightarrow+\infty} \int_{0}^{s} \sigma^{\lambda} K(\sigma) \mathrm{d} \sigma=+\infty
$$

Setting $\lambda=0$ in Moore statement we recover Ambrose-Wintner theorem, which improves on Fite theorem quoted in Remark 2.8. As Remark 1.22 shows, the result is false if $\lambda=1$.

Moore result, although sharp from many points of view, requires that the negative part of $K$ is, loosely speaking, globally smaller than the positive part. This is the essence of the existence of the limit in (2.13). In Chapter 5, in a slightly different context, we will prove an oscillation result that allows $K$ to have a relevant non-positive part. In particular, see Section 5.2 for a detailed discussion.

We shift our attention to the first problem. A striking result in this direction is due to E. Calabi [Cal67]. Since the techniques are very close to those presented in Section 2.2, we provide a complete proof of the next

Theorem 2.10 ([Cal67], Theorems 1 and 2). Let $(M,\langle\rangle$,$) be a complete Rie-$ mannian manifold of dimension $m$, and assume that Ricc $\geq 0$ on $M$. For each unit 
speed geodesic $\gamma$, define

$$
K_{\gamma}(s)=\frac{\operatorname{Ricc}\left(\gamma^{\prime}, \gamma^{\prime}\right)(s)}{m-1}
$$

Suppose that, for every $\gamma$ issuing from some origin o, there exists $0<a<b$ (possibly depending on $\gamma$ ) such that

$$
\int_{a}^{b} \sqrt{K_{\gamma}(\sigma)} \mathrm{d} \sigma>\left\{\left(1+\frac{1}{2} \log \frac{b}{a}\right)^{2}-1\right\}^{1 / 2} .
$$

Then, $M$ is compact with finite fundamental group. In particular this happens if, for every $\gamma$,

$$
\limsup _{s \rightarrow+\infty}\left(\int_{0}^{s} \sqrt{K_{\gamma}(\sigma)} \mathrm{d} \sigma-\frac{1}{2} \log s\right)=+\infty .
$$

Proof. By Theorem 2.1, it is enough to prove that a solution $g$ of

$$
\left\{\begin{array}{l}
g^{\prime \prime}+K_{\gamma} g=0 \quad \text { on } \mathbb{R}^{+} \\
g(0)=0, \quad g^{\prime}(0)=1
\end{array}\right.
$$

has a first zero. Suppose by contradiction that $g>0$ on $\mathbb{R}^{+}$. Then, $\phi=g^{\prime} / g$ solves

$$
\left\{\begin{array}{l}
\phi^{\prime}+\phi^{2}=-K_{\gamma} \leq 0 \text { on } \mathbb{R}^{+} \\
\phi(s)=\frac{1}{s}+O(1) \quad \text { as } \quad s \rightarrow 0^{+} .
\end{array}\right.
$$

Set $t=t(s)=e^{s}$ and define $w(s)$ on the whole $\mathbb{R}$ by means of

$$
\phi(t)=e^{-s}\left(w(s)+\frac{1}{2}\right)
$$

Then, $w(s)$ solves

$$
w^{\prime}+w^{2} \leq \frac{1}{4} \quad \text { on } \mathbb{R}
$$

We claim that $w(s) \in[-1 / 2,1 / 2]$ on $\mathbb{R}$. Indeed, suppose by contradiction that, for some $s_{0} \in \mathbb{R}, w\left(s_{0}\right)<-1 / 2$. Let $\psi_{c}$ be the general solution of $\psi_{c}^{\prime}+\psi_{c}^{2}=B^{2}$ on $\mathbb{R}$, where $c \in \mathbb{R}$ is a parameter and $B>0$. Depending on the initial data (i.d.), the expression of $\psi_{c}$ is given by

$$
\psi_{c}(s)= \begin{cases}B \operatorname{coth}(B(s-c)) & \text { if the i.d. is }<-B \text { or }>B, \\ \pm B & \text { if the i.d. is } \pm B, \\ B \tanh (B(s-c)) & \text { if the i.d. is in }(-B, B) .\end{cases}
$$

Set $B=1 / 2$. Since $w\left(s_{0}\right)<-1 / 2$, we can choose $c$ sufficiently large that the function

$$
\psi_{c}=\frac{1}{2} \operatorname{coth}\left(\frac{s-c}{2}\right) \quad \text { satisfies } \quad w\left(s_{0}\right) \leq \psi_{c}\left(s_{0}\right)<-\frac{1}{2} .
$$

By the Riccati comparison 1.12, $w \leq \psi_{c}$ on $\left[s_{0},+\infty\right)$, and since $\psi_{c} \rightarrow-\infty$ as $s \rightarrow c^{-}$this contradicts the fact that $w$ is defined on $\mathbb{R}$. The case $w\left(s_{0}\right)>1 / 2$ can be treated similarly. 
Choose now $0<a<b$, and set $a^{\prime}=\log a, b^{\prime}=\log b$. Then, changing variables according to $\sigma=\sigma(\xi)=e^{\xi}$ we get

$$
\int_{a}^{b} \sqrt{K_{\gamma}(\sigma)} \mathrm{d} \sigma=\int_{a}^{b} \sqrt{-\phi^{\prime}(\sigma)-\phi^{2}(\sigma)} \mathrm{d} \sigma=\int_{a^{\prime}}^{b^{\prime}} \sqrt{-w^{\prime}(\xi)-w^{2}(\xi)+\frac{1}{4}} \mathrm{~d} \xi
$$

On the other hand, using Cauchy-Schwarz inequality, $w \in[-1 / 2,1 / 2]$ and the definition of $a^{\prime}, b^{\prime}$ we deduce

$$
\begin{aligned}
\left(\int_{a^{\prime}}^{b^{\prime}} \sqrt{-w^{\prime}(\xi)-w^{2}(\xi)+\frac{1}{4}} \mathrm{~d} \xi\right)^{2} & \leq\left(b^{\prime}-a^{\prime}\right) \int_{a^{\prime}}^{b^{\prime}}\left(-w^{\prime}(\xi)-w^{2}(\xi)+\frac{1}{4}\right) \mathrm{d} \xi \\
& \leq\left(b^{\prime}-a^{\prime}\right)\left[\left|-w\left(b^{\prime}\right)+w\left(a^{\prime}\right)\right|+\frac{1}{4}\left(b^{\prime}-a^{\prime}\right)\right] \\
& \leq\left[\frac{1}{2}\left(b^{\prime}-a^{\prime}\right)+1\right]^{2}-1 \\
& =\left[\frac{1}{2} \log \frac{b}{a}+1\right]^{2}-1 .
\end{aligned}
$$

Combining (2.20) and (2.21) we contradict the assumption (2.15). That (2.16) implies (2.15) is immediate.

REMARK 2.11. The conclusions of Theorem 2 of [Cal67] are slightly more general than those presented above. Using this improved form, one can easily get that under condition (2.16) the solution $g$ of (2.17) indeed oscillates. In the next sections, we shall call (2.16) the Calabi oscillation criterion.

In the subsequent years, it seems to the authors that no substantial new achievements have appeared besides the very recent result in $[\mathbf{M R V}]$, which we are going to describe in a moment. Nevertheless, it shall be observed that the problem of obtaining Myers type compactness theorems under the presence of a suitably small amount of negative Ricci curvature has already been a flourishing field of research, for which we refer the reader to [Wu91], [ER91], [RY94] and the references therein. The techniques employed in these papers are of various nature and go much beyond the purpose of the present work, in particular neither of them relies on oscillation type results for a linear ODE. We stress that, however, the method in [Wu91] via Jacobi fields is indeed closely related to our approach. Getting back to $[$ MRV], P. Mastrolia, M. Rimoldi and G. Veronelli have followed the ideas on the proof of Calabi result to give the first condition in finite form for the existence of a first zero of $g^{\prime \prime}+K g=0$ when $K$ is only assumed to satisfy $K \geq-B^{2}$, for some $B \geq 0$. Applied to the compactness problem for Riemannian manifolds, their inequality improves on the application of Nehari condition ([Neh57], p.432 (8)), which requires $K \geq 0$. As we will see in the next section, the techniques used for Theorems 2.10 and 2.12 will also be a key tool in estimating the essential spectrum of the Laplacian. We state the result in [MRV] in geometric form.

Theorem 2.12 ([MRV], Theorem 5). Let $(M,\langle\rangle$,$) be a complete Riemannian$ manifold of dimension $m \geq 1$ satisfying

$$
\operatorname{Ricc} \geq-(m-1) B^{2}\langle,\rangle,
$$

for some $B \geq 0$. For every unit speed geodesic $\gamma$ issuing from $o \in M$, let $K_{\gamma}$ be as in (2.14). Suppose that, for each such $a \gamma$, there exist $0<a<b$ and $\lambda \neq 1$ for 
which either

$$
\int_{a}^{b} s K_{\gamma}(s) \mathrm{d} s>B\left\{b+a \frac{e^{2 B a}+1}{e^{2 B a}-1}\right\}+\frac{1}{4} \log \left(\frac{b}{a}\right)
$$

or

$$
\int_{a}^{b} s^{\lambda} K_{\gamma}(s) \mathrm{d} s>B\left\{b^{\lambda}+a^{\lambda} \frac{e^{2 B a}+1}{e^{2 B a}-1}\right\}+\frac{\lambda^{2}}{4(1-\lambda)}\left\{a^{\lambda-1}-b^{\lambda-1}\right\}
$$

holds (if $B=0$, this has to be intended in a limit sense). Then, $M$ is compact with finite fundamental group.

Proof. Again, we prove that a solution $g$ of (2.17) has a first zero. Suppose by contradiction that $g>0$ on $\mathbb{R}^{+}$. Then, setting $\phi=g^{\prime} / g$, by assumption (2.22) $\phi$ solves

$$
\left\{\begin{array}{l}
\phi^{\prime}+\phi^{2}=-K_{\gamma} \leq B^{2} \quad \text { on } \mathbb{R}^{+} \\
\phi(s)=\frac{1}{s}+O(1) \quad \text { as } s \rightarrow 0^{+} .
\end{array}\right.
$$

We compare $\phi$ with the general solution $\psi_{c}$ of $\psi_{c}^{\prime}+\psi_{c}^{2}=B^{2}$ given by (2.19). From Proposition 1.13 with the choices $\alpha=1, G=B^{2}, h_{1}=\phi$ and $h_{2}=\psi_{0}$ we deduce

$$
\phi(s) \leq \psi_{0}(s)=B \operatorname{coth}(B s)=B \frac{e^{2 B s}+1}{e^{2 B s}-1} \quad \text { on } \mathbb{R}^{+} .
$$

Moreover, with the same technique used in the proof of Theorem 2.10 to show that $w(s) \in[-1 / 2,1 / 2]$, we get the bound $\phi \geq-B$ on $\mathbb{R}^{+}$. Now, consider the case $\lambda \neq 1$, and choose any $0<a<b$. Integrating by parts and using the estimate on $\phi$ we deduce

$$
\begin{aligned}
\int_{a}^{b} s^{\lambda} K_{\gamma}(s) \mathrm{d} s & =\int_{a}^{b} s^{\lambda}\left(-\phi^{\prime}(s)-\phi^{2}(s)\right) \mathrm{d} s \\
& =\int_{a}^{b}\left[-\left(s^{\lambda} \phi(s)\right)^{\prime}-s^{\lambda}\left(\phi(s)-\frac{\lambda}{2 s}\right)^{2}+\frac{\lambda^{2}}{4} s^{\lambda-2}\right] \mathrm{d} s \\
& \leq-b^{\lambda} \phi(b)+a^{\lambda} \phi(a)+\frac{\lambda^{2}}{4(\lambda-1)}\left(b^{\lambda-1}-a^{\lambda-1}\right) \\
& \leq\left[b^{\lambda} B+a^{\lambda} \psi_{0}(a)\right]+\frac{\lambda^{2}}{4(\lambda-1)}\left(b^{\lambda-1}-a^{\lambda-1}\right),
\end{aligned}
$$

contradicting assumption (2.24), as desired. The case $\lambda=1$ is analogous, and $B=0$ follows by taking the limit as $B \rightarrow 0$.

REMARK 2.13. With a slight improvement of the above technique, one can also give an upper bound for the diameter of $M$. For details, we refer the reader to [MRV], Remark 18.

The method developed in Theorems 2.10 and 2.12 seems to be hardly generalizable to cover, for instance, the case

$$
K(s) \geq-B^{2}\left(1+s^{2}\right)^{\alpha / 2}, \quad B \geq 0, \alpha \geq-2,
$$

mainly because of the lack of a manageable form of the general solution of $\psi_{c}^{\prime}+\psi_{c}^{2}=$ $B^{2}\left(1+r^{2}\right)^{\alpha / 2}$. For this reason, a different approach shall be adopted. Note that, when $\alpha>0,(2.25)$ allows a lower bound that diverges as $s \rightarrow+\infty$; therefore, proving the existence of zeroes of $g$ when $K$ satisfies (2.25) will lead to a nontrivial improvement of Theorems 2.10 and 2.12 . 
In Section 5.1, we will generalize Calabi oscillation criterion (2.16) for $g^{\prime \prime}+K g=0$ to the case when $K$ has only to satisfy $K(s) \geq-B^{2} s^{\alpha}$, for some $\alpha \geq-2$ and $s$ sufficiently large. Furthermore, under the mild requirement (2.25), we will also provide a condition in finite form for the existence of a first zero of $g$. When $\alpha=0$, this condition does not overlap neither with (2.15) nor with (2.24). As we will see in Section 5.6, the negative part of the potential $K$ has a peculiar role. In substance, it enters the problem as some sort of weight for the manifold.

\subsection{The spectrum of the Laplacian on complete manifolds}

The study of the relations between the spectrum of $-\Delta$ on complete, noncompact manifolds and the geometric data (e.g. curvatures, volume growth) has been the core of an active area of research for the last four decades. Among the various interesting problems, a basic question concerns the characterization of the discrete and the continuous part of $\sigma(-\Delta)$. For this purpose, estimates on $\Delta r$ are useful, so that the ODE theory of Riccati and linear equations naturally comes into play. To introduce the argument, we give here a brief presentation of some of the principal results in the literature that shall be useful in the sequel, and we concentrate on proofs whenever the approach is close to the spirit of this paper. In the next chapters, we shall apply our techniques and results to recover and, possibly, to generalize some of the theorems described in this section.

We begin with the following simple estimate appearing in [Bro81] and [RRV97].

Proposition 2.14. Let $(M,\langle\rangle$,$) be a complete Riemannnian manifold with a$ pole $o$, and let $r(x)$ be the distance function from o. Suppose that $\Delta r \geq 0$ on $M$. Then,

$$
\lambda_{1}^{-\Delta}(M) \geq \frac{1}{4} \inf _{M}(\Delta r)^{2}, \quad \inf \sigma_{\mathrm{ess}}(-\Delta) \geq \frac{1}{4} \liminf _{r(x) \rightarrow+\infty}(\Delta r(x))^{2} .
$$

Proof. Let $\Omega \subset M$ be a open set. By the first Green formula we deduce, for every smooth domain $D \Subset \Omega$,

$$
\operatorname{vol}(D) \inf _{\Omega} \Delta r \leq \int_{D} \Delta r=\int_{\partial D}\langle\nabla r, \nu\rangle \leq \operatorname{vol}(\partial D) .
$$

Hence, indicating with $c(\Omega)$ the Cheeger constant of $\Omega$, by Cheeger inequality, [Che70], and the assumption $\Delta r \geq 0$ we get

$$
\lambda_{1}^{-\Delta}(\Omega) \geq \frac{c(\Omega)^{2}}{4}=\frac{1}{4}\left(\inf _{D \Subset \Omega} \frac{\operatorname{vol}(\partial D)}{\operatorname{vol}(D)}\right)^{2} \geq \frac{1}{4}\left(\inf _{\Omega} \Delta r\right)^{2} .
$$

The first inequality in (2.26) follow by choosing $\Omega=M$, while for the second inequality we consider $\Omega=M \backslash B_{r}$, we let $r \rightarrow+\infty$ and we use Theorem 1.38.

Remark 2.15. Clearly, in estimating inf $\sigma_{\text {ess }}(-\Delta)$ it is enough to assume $\Delta r \geq$ 0 only outside some compact set. A sufficient condition for $\Delta r>0$ to hold on $M \backslash\{o\}$ has been provided by Corollary 1.23.

The characterization of the essential spectrum has been studied by many authors, notably H. Donnelly [Don81b], H. Donnelly and P. Li [LD79], J.F. Escobar and A. Freire [EF92], J. Li [Li94] and H. Kumura [Kum97], [Kum05]. The next Theorem, due to Donnelly, has been refined by Kumura. The proof below is a simplified version of that appearing in [Kum97]. 
Theorem 2.16 ([Kum97], Theorem 1.2 and [Don81b]). Let $(M,\langle\rangle$,$) be a$ complete, non-compact Riemannian manifold with a pole o. Suppose that $\Delta r(x) \rightarrow c$ as $r(x) \rightarrow+\infty$, for some $c \in \mathbb{R}_{0}^{+}$. Then, $\sigma_{\mathrm{ess}}(-\Delta)=\left[c^{2} / 4,+\infty\right)$.

Proof. Since $\sigma_{\text {ess }}(-\Delta) \subset \mathbb{R}_{0}^{+}$is closed, applying Proposition 2.14 and Remark 2.15 , it is enough to show that each $\lambda>c^{2} / 4$ is in the essential spectrum of $-\Delta$. To do so, we shall exhibit a characteristic sequence for $\lambda$, that will be obtained by comparing $M$ with a suitable sequence of manifolds $\left(M_{k}, \mathrm{~d} s_{k}^{2}\right)$. Since $M$ has a pole, we can consider global geodesic coordinates $(r, \theta)$, where with the symbols $\theta$, $\Omega_{\theta}$ we respectively denote a local coordinate system and the volume form of $\mathbb{S}^{m-1}$, $m=\operatorname{dim}(M)$. Let $\omega=\omega(r, \theta)$ be the volume density, that is, the volume element of $M$ can be expressed as $\mathrm{d} V=\omega \mathrm{d} r \wedge \Omega_{\theta}$. Define

$$
\lambda_{c}=\left(\lambda-\frac{c^{2}}{4}\right)^{-1 / 2} \text {. }
$$

First, we construct inductively a sequence $\left\{u_{k}\right\} \subset \operatorname{Lip}_{c}(M)$ close to a characteristic one for $\lambda$. Fix $k>0$. For each $r_{k}>0$, to be specified later, consider the interval $I_{k}=\left[r_{k}, r_{k}+2 \pi \lambda_{c}\right]$. Define $M_{k}$ to be $\left[r_{k},+\infty\right) \times \mathbb{S}^{m-1}$ equipped with the metric $\mathrm{d} s_{k}^{2}$ given, in polar coordinates $(\rho, \theta)$, by

$$
\mathrm{d} s_{k}^{2}=\mathrm{d} \rho^{2}+\omega_{k}(\rho, \theta)^{\frac{2}{m-1}} \mathrm{~d} \theta^{2},
$$

with $\omega_{k}(\rho, \theta)=\exp \left\{c\left(\rho-r_{k}\right)\right\} \omega\left(r_{k}, \theta\right)$ the volume density of $M_{k}$, and let $\mathrm{d} V_{k}=$ $\omega_{k} \mathrm{~d} \rho \wedge \Omega_{\theta}$ be the volume form. A computation shows that the function

$$
z_{k}(\rho)=\exp \left\{-\frac{c\left(\rho-r_{k}\right)}{2}\right\} \sin \left(\frac{\rho-r_{k}}{\lambda_{c}}\right)
$$

satisfies $z_{k}^{\prime \prime}+c z_{k}^{\prime}=-\lambda z_{k}$ on $\left[r_{k},+\infty\right)$. Hence, $w_{k}(\rho, \theta)=z_{k}(\rho)$ is a solution of $\Delta w_{k}+\lambda w_{k}=0$ on $M_{k}$. From the assumption $\Delta r \rightarrow c$ as $r(x) \rightarrow+\infty$, for every $k$ we can suppose that $r_{k}$ is chosen in such a way that

$$
|\Delta r(x)-c|<\frac{1}{k} \quad \text { on } M \backslash B_{r_{k}} .
$$

Using (1.48), we deduce that

$$
c-\frac{1}{k} \leq \frac{\partial_{r} \omega}{\omega} \leq c+\frac{1}{k}
$$

and integrating on $\left[r_{k}, \rho\right] \subset I_{k}$ we get

$$
\omega\left(r_{k}, \theta\right) \exp \left\{\left(c-\frac{1}{k}\right)\left(\rho-r_{k}\right)\right\} \leq \omega(\rho, \theta) \leq \omega\left(r_{k}, \theta\right) \exp \left\{\left(c+\frac{1}{k}\right)\left(\rho-r_{k}\right)\right\} .
$$

Using the definition of $\omega_{k}(\rho, \theta)$ and the fact that $\rho-r_{k} \leq\left|I_{k}\right|=2 \pi \lambda_{c}$, the following inequalities hold:

$$
\omega_{k}(\rho, \theta) \exp \left\{-\frac{2 \pi \lambda_{c}}{k}\right\} \leq \omega(\rho, \theta) \leq \omega_{k}(\rho, \theta) \exp \left\{\frac{2 \pi \lambda_{c}}{k}\right\} .
$$

Up to choosing $k$ sufficiently large, we have therefore

$$
\frac{1}{2} \omega_{k} \leq \omega \leq 2 \omega_{k} \quad \text { on } I_{k} \times \mathbb{S}^{m-1} .
$$

Define $u_{k}(x)=z_{k}(r(x)) \chi_{A_{k}}$, where $\chi_{A_{k}}$ is the characteristic function of the annulus $A_{k}=I_{k} \times \mathbb{S}^{m-1} \subset M$. For notational convenience, we agree on denoting with $A_{k}$ 
also the annulus $I_{k} \times \mathbb{S}^{m-1} \subset M_{k}$. From the properties of $z_{k},(2.29)$ and Green formula on $M_{k}$ we deduce that

$$
\begin{aligned}
\left\|\Delta u_{k}+\lambda u_{k}\right\|_{L^{2}\left(A_{k}\right)}^{2} & =\int_{A_{k}}\left|z_{k}^{\prime \prime}+z_{k}^{\prime} \Delta r+\lambda z_{k}\right|^{2} \mathrm{~d} V \leq \int_{A_{k}}\left|z_{k}^{\prime}\right|^{2}|\Delta r-c|^{2} \omega \mathrm{d} r \wedge \Omega_{\theta} \\
& \leq \frac{2}{k^{2}} \int_{A_{k}}\left|z_{k}^{\prime}\right|^{2} \omega_{k} \mathrm{~d} r \wedge \Omega_{\theta}=\frac{2}{k^{2}} \int_{A_{k}}\left|\nabla w_{k}\right|^{2} \mathrm{~d} V_{k} \\
& =-\frac{2}{k^{2}} \int_{A_{k}} w_{k} \Delta w_{k} \mathrm{~d} V_{k}=\frac{2 \lambda}{k^{2}} \int_{A_{k}} w_{k}^{2} \mathrm{~d} V_{k} \\
& \leq \frac{4 \lambda}{k^{2}} \int_{A_{k}} z_{k}^{2} \omega \mathrm{d} r \wedge \Omega_{\theta}=\frac{4 \lambda}{k^{2}}\left\|u_{k}\right\|_{L^{2}\left(A_{k}\right)}^{2}
\end{aligned}
$$

Normalizing $u_{k}$ in $L^{2}$, we have that

$$
\left\|u_{k}\right\|_{L^{2}}=1, \quad \text { while } \quad\left\|\Delta u_{k}+\lambda u_{k}\right\|_{L^{2}\left(A_{k}\right)} \leq \frac{4 \lambda}{k^{2}} \rightarrow 0 \quad \text { as } k \rightarrow+\infty .
$$

Observe that, up to choosing $r_{k}$ sufficiently large, we can suppose that the support of $u_{k}$ is disjoint from that of $u_{1}, \ldots, u_{k-1}$. Now, we approximate $u_{k}$. Since $-\Delta$ is essentially self-adjoint on $C_{c}^{\infty}\left(A_{k}\right)$, for every fixed $k$, by (1.77), there exists $\left\{u_{k, j}\right\}_{j} \subset C_{c}^{\infty}\left(A_{k}\right)$ such that $u_{k, j} \rightarrow u_{k}$ in $L^{2}$ and $\Delta u_{k, j} \rightarrow \Delta u_{k}$ in $L^{2}$. By (2.31) and a Cantor diagonal argument, the functions $v_{k}=u_{k, k}$ have pairwise disjoint support and satisfy, for some $C>0$,

$$
\left\{v_{k}\right\} \subset C_{c}^{\infty}(M), \quad\left\|v_{k}\right\|_{L^{2}} \geq C, \quad\left\|\Delta v_{k}+\lambda v_{k}\right\|_{L^{2}} \rightarrow 0 \quad \text { as } k \rightarrow+\infty,
$$

that is, $\left\{v_{k}\right\}$ is the required characteristic sequence for $\lambda$.

Very recently, in $\left[\right.$ Kum05] the author points out that, for property $\sigma_{\text {ess }}(-\Delta) \subset$ $\left[c^{2} / 4,+\infty\right)$ to hold, the requirement $\Delta r \rightarrow c$ as $r(x) \rightarrow+\infty$ can be weakened. Indeed, up to the mild further requirements $\operatorname{vol}(M)=+\infty$ and $\Delta r \geq-\widehat{c}$ outside some compact set, for some constant $\widehat{c}>0$, it is enough that

$$
\|\Delta r-c\|_{L^{2}\left(M \backslash B_{r}\right)} \rightarrow 0 \quad \text { as } r \rightarrow+\infty .
$$

Via the Petersen-Wei method in [PW97], (2.32) is granted by an $L^{p}$ control of the type

$$
\frac{1}{\operatorname{vol}\left(B_{r}\right)} \int_{B_{r}}\left(\frac{c^{2}}{m-1}-\operatorname{Ricc}(\nabla r, \nabla r)\right)_{+}^{p} \mathrm{~d} V \longrightarrow 0 \quad \text { as } r \rightarrow+\infty,
$$

for some $p>m / 2$. Since the techniques used to prove this interesting result are somehow beyond the scope of the present paper, we will not elaborate on this subject. The condition $\Delta r \rightarrow c$ of Proposition 2.16 can be achieved via Riccati comparisons, under suitable control on the radial sectional or Ricci curvatures. This is the content of the following Corollary that collects some results of most of the authors cited above. The technique of the proofs follows the same type of argument of Theorem 2.12.

Corollary 2.17. Let $(M,\langle\rangle$,$) be a manifold with a pole o$.

(i) ([Don81b]) Let $K_{\mathrm{rad}}$ satisfies $K_{\mathrm{rad}}(x) \leq-G(r(x))$, for some $G \in C^{0}\left(\mathbb{R}_{0}^{+}\right)$ such that

$$
G_{-} \in L^{1}(+\infty), \quad r \int_{r}^{+\infty} G_{-}(\sigma) \mathrm{d} \sigma \leq \frac{1}{4} \quad \text { on } \quad \mathbb{R}_{0}^{+},
$$


and suppose that $K_{\text {rad }}(x) \rightarrow-B^{2}$ as $r(x) \rightarrow+\infty$, for some $B \geq 0$. Then,

$$
\sigma_{\mathrm{ess}}(-\Delta)=\left[\frac{B^{2}(m-1)^{2}}{4},+\infty\right)
$$

(ii) ([LD79]) Let $K_{\mathrm{rad}}$ and $G(r)$ satisfy the assumptions of item $(i)$. If $K_{\mathrm{rad}}(x) \rightarrow-\infty$ as $x$ diverges, then $-\Delta$ has discrete spectrum.

(iii) ([Li94], [Kum97], [EF92]) Suppose that the Ricci curvature satisfies

$$
\operatorname{Ricc}(\nabla r, \nabla r) \geq-(m-1) G(r),
$$

for some $0 \leq G(r) \rightarrow 0$ as $r \rightarrow+\infty$. Then, $\sigma_{\text {ess }}(-\Delta)=\mathbb{R}_{0}^{+}$.

Proof. ( $i$ ) By Theorem 2.16, it is enough to show that $\Delta r \rightarrow(m-1) B$ as $r \rightarrow+\infty$. Without loss of generality, we can assume that $-G(r)$ is the supremum of the radial sectional curvatures at point $x \in \partial B_{r}$. Define $-G_{i}(r)$ to be the infimum of the radial sectional curvatures of points $x \in \partial B_{r}$. By definition, for every $x \in M$, $X \in \nabla r_{x}^{\perp}$

$$
-G_{i}(r(x)) \leq K_{\mathrm{rad}}(X) \leq-G(r(x)) \quad \text { on } \mathbb{R}_{0}^{+},
$$

and $G_{i}, G \rightarrow B^{2}$ as $r \rightarrow+\infty$. By Proposition 1.21, and by Sturm argument, it follows that the solutions $g_{i}, g$ of

$$
\left\{\begin{array} { l } 
{ g ^ { \prime \prime } - G g = 0 } \\
{ g ( 0 ) = 0 , \quad g ^ { \prime } ( 0 ) = 1 }
\end{array} \quad \left\{\begin{array}{l}
g_{i}^{\prime \prime}-G_{i} g_{i}=0 \\
g_{i}(0)=0, \quad g_{i}^{\prime}(0)=1
\end{array}\right.\right.
$$

are positive and increasing on $\mathbb{R}^{+}$, hence $\phi=(m-1) g^{\prime} / g, \phi_{i}=(m-1) g_{i}^{\prime} / g_{i}$ are positive solutions of

$$
\phi^{\prime}+\frac{\phi^{2}}{m-1}=(m-1) G, \quad \phi_{i}^{\prime}+\frac{\phi_{i}^{2}}{m-1}=(m-1) G_{i} .
$$

By the Laplacian comparison Theorems 1.17 and 1.19 we deduce that

$$
(0<) \quad \phi(r(x)) \leq \Delta r(x) \leq \phi_{i}(r(x)) \quad \text { on } \mathbb{R}^{+} .
$$

To prove that $\Delta r(x) \rightarrow(m-1) B$ as $r(x) \rightarrow+\infty$, it is enough to show that $\phi, \phi_{i} \rightarrow(m-1) B$ as $r \rightarrow+\infty$. For convenience, we consider $\eta=\phi /(m-1)$, $\eta_{i}=\phi_{i} /(m-1)$, and we prove that $\eta, \eta_{i} \rightarrow B$. Note that

$$
\eta^{\prime}+\eta^{2}=G, \quad \eta_{i}^{\prime}+\eta_{i}^{2}=G_{i}, \quad \eta \leq \eta_{i} .
$$

We deal with the case $B>0$, the case $B=0$ being analogous. For every $\varepsilon>0$ small enough, let $r_{\varepsilon}$ be such that $G, G_{i} \in\left(-(B+\varepsilon)^{2},-(B-\varepsilon)^{2}\right)$ on $M \backslash B_{r_{\varepsilon}}$. Set for convenience $B_{\varepsilon}=B-\varepsilon, B^{\varepsilon}=B+\varepsilon$, and denote with $\psi, \psi_{i}$ the solutions of the following Cauchy problems on $\left[r_{\varepsilon},+\infty\right)$ :

$$
\left\{\begin{array} { l } 
{ \psi ^ { \prime } + \psi ^ { 2 } = ( B _ { \varepsilon } ) ^ { 2 } } \\
{ \psi ( r _ { \varepsilon } ) = \eta ( r _ { \varepsilon } ) }
\end{array} \quad \left\{\begin{array}{l}
\psi_{i}^{\prime}+\psi_{i}^{2}=\left(B^{\varepsilon}\right)^{2} \\
\psi_{i}\left(r_{\varepsilon}\right)=\eta_{i}\left(r_{\varepsilon}\right)
\end{array}\right.\right.
$$

Then, by the Riccati comparison 1.12, we get $\psi \leq \eta$ and $\eta_{i} \leq \psi_{i}$ on $\left[r_{\varepsilon},+\infty\right)$. Taking into account the form of the general solution (2.19) of the $\mathrm{ODE} \psi^{\prime}+\psi^{2}=B^{2}$, and observing that the initial conditions $\eta\left(r_{\varepsilon}\right), \eta_{i}\left(r_{\varepsilon}\right)$ are positive numbers, we get the chain of inequalities

$$
\begin{aligned}
B_{\varepsilon} & =\lim _{s \rightarrow+\infty} \psi(s) \leq \liminf _{s \rightarrow+\infty} \eta(s) \leq \limsup _{s \rightarrow+\infty} \eta(s) \\
& \leq \liminf _{s \rightarrow+\infty} \eta_{i}(s) \leq \limsup _{s \rightarrow+\infty} \eta_{i}(s) \leq \lim _{s \rightarrow+\infty} \psi_{i}(s)=B^{\varepsilon} .
\end{aligned}
$$


The claim $\eta, \eta_{i} \rightarrow B$ as $s \rightarrow+\infty$ is proved letting $\varepsilon \rightarrow 0$.

(ii) By Proposition 2.14 and the min-max theorem, it is enough to prove that $\Delta r(x) \rightarrow+\infty$ as $r(x) \rightarrow+\infty$. Let $G, g, \phi, \eta$ be as in the proof of item $(i)$. As already observed, by assumption (2.33) the function $g$ is positive and increasing, and by $(2.34)$

$$
\Delta r \geq \phi(r)=(m-1) \eta(r),
$$

where $\eta$ is a positive solution of $\eta^{\prime}+\eta^{2}=G$. We prove that $\eta$ diverges as $r \rightarrow+\infty$. By the assumption $K_{\mathrm{rad}} \rightarrow-\infty$, for every $B>0$ we can choose $r_{B}>0$ sufficiently large that $G \geq B^{2}$ on $\left[r_{B},+\infty\right)$. Comparing $\eta$ with a solution of $\psi^{\prime}+\psi^{2}=B^{2}$ in (2.19) with the initial condition $\psi\left(r_{B}\right)=\eta\left(r_{B}\right)>0$ we deduce that $\psi \leq \eta$, thus

$$
\liminf _{r \rightarrow+\infty} \eta(r) \geq \lim _{r \rightarrow+\infty} \psi(r)=B
$$

and the sought follows letting $B \rightarrow+\infty$.

(iii) By Theorem 2.16, it is enough to prove that $\Delta r \rightarrow 0$ as $r \rightarrow+\infty$. By the Laplacian comparison Theorem 1.19

$$
\Delta r \leq(m-1) g^{\prime}(r) / g(r)=(m-1) \eta(r),
$$

where $\eta(r)$ solves $\eta^{\prime}+\eta^{2}=G$. Fix $\varepsilon>0$, and let $r_{\varepsilon}>0$ be such that $G \in\left[0, \varepsilon^{2}\right)$ on $\left[r_{\varepsilon},+\infty\right)$. Let $\gamma: \mathbb{R}_{0}^{+} \rightarrow M$ be a ray issuing from $o$, and define

$$
u_{\gamma}(s)=\frac{\Delta r \circ \gamma(s)}{m-1}
$$

By the Riccati comparison 1.12, formula (1.47), and $\operatorname{Ricc}(\nabla r, \nabla r) \geq-(m-1) \varepsilon^{2}$ on $\left[r_{\varepsilon},+\infty\right)$, we get that $u_{\gamma} \leq \psi_{\gamma}$ on $\left[r_{\varepsilon},+\infty\right)$, where $\psi_{\gamma}$ solves

$$
\left\{\begin{array}{l}
\psi_{\gamma}^{\prime}+\psi_{\gamma}^{2}=\varepsilon^{2} \quad \text { on }\left[r_{\varepsilon},+\infty\right) \\
\psi_{\gamma}\left(r_{\varepsilon}\right)=\Delta r\left(\gamma\left(r_{\varepsilon}\right)\right) .
\end{array}\right.
$$

We claim that $u_{\gamma}(s) \geq-\varepsilon$ on $\left[r_{\varepsilon},+\infty\right)$. Indeed, if by contradiction $u_{\gamma}\left(r_{1}\right)<$ $-\varepsilon$ for some $r_{1}>r_{\varepsilon}$, then by (2.19) (with $\varepsilon$ replacing $B$ ) the solution of (2.38) with initial condition $\psi_{\gamma}\left(r_{1}\right)=u\left(r_{1}\right)$ tends to $-\infty$ in finite time. Thus, from the Riccati comparison, this contradicts the fact that $u_{\gamma}$ is defined on $\left[r_{\varepsilon},+\infty\right)$. By the arbitrariness of $\gamma, \Delta r \geq-(m-1) \varepsilon$ on $\left[r_{\varepsilon},+\infty\right)$. Next, again by the Riccati comparison $1.12, \eta \leq \psi$, where $\psi$ is a solution of

$$
\left\{\begin{array}{l}
\psi^{\prime}+\psi^{2}=\varepsilon^{2} \quad \text { on }\left[r_{\varepsilon},+\infty\right) \\
\psi\left(r_{\varepsilon}\right)=\eta\left(r_{\varepsilon}\right) .
\end{array}\right.
$$

Since $\eta\left(r_{\varepsilon}\right) \geq-\varepsilon$, the explicit solution $\psi$ of $(2.39)$ tends either to $\varepsilon$ (if $\eta\left(r_{\varepsilon}\right)>-\varepsilon$ ) or to $-\varepsilon$ (if $\eta\left(r_{\varepsilon}\right)=-\varepsilon$ ). Summarizing, we have showed that, on $M \backslash B_{r_{\varepsilon}}$

$$
-\varepsilon \leq \Delta r(x) \leq(m-1) \eta(r) \rightarrow \varepsilon \text { or }-\varepsilon \quad \text { as } r \rightarrow+\infty,
$$

and $\Delta r \rightarrow 0$ follows by the arbitrariness of $\varepsilon$.

REMARK 2.18. It is interesting to observe that, for (iii), the assumption (2.33) is not needed. As a matter of fact, this requirement only guarantees that the lower bound $\phi$ in (2.34) is positive. By Riccati comparison and the explicit formula (2.19), $\phi>0$ is enough to ensure that $\psi$ in (2.35) tends to $B_{\varepsilon}$ and not to $-B_{\varepsilon}$ as $r$ diverges. This allows to conclude that $\Delta r \rightarrow(m-1) B$ by (2.36). Loosely speaking, if $B=0$ we have no gap between $B_{\varepsilon}$ and $-B_{\varepsilon}$, so there is no need of (2.33). 
REMARK 2.19. We stress that there exist explicit conditions which are both necessary and sufficient to ensure the discreteness of the spectrum of $\Delta$ on a manifold. Such conditions are the results of various approaches, whose ideas are generally unrelated to those described in this paper. For this reason, without any further mention we refer the interested reader to [Kle88] and [CM11] for a detailed insight.

Next, we spend a few words about the discrete spectrum. When $\sigma_{\text {ess }}(-\Delta) \neq \emptyset$, we can ask whether $\sigma_{\text {disc }}(-\Delta)$ is empty or not. A celebrated theorem of S.T. Yau [Yau76] states that a complete manifold with infinite volume does not support any non-zero $L^{2}$ harmonic function, i.e. the eigenspace associated to the zero eigenvalue is trivial. Furthermore, if $\operatorname{vol}(M)<+\infty$, the space of $L^{2}$ harmonic functions is the 1-dimensional space of constants. For eigenvalues $\lambda>0$, either in the discrete or in the essential spectrum, things are much more complicated. Among the techniques developed to prove non-existence of $L^{2}$ eigenfunctions of $-\Delta$ related to $\lambda>0$, Rellich type integral identities turned out to be extremely useful. We suggest the interested reader to consult [EF92], [DG92], [Don81a], [Don90], [Esc85] and the references therein. The next theorem collects some of the results in these papers.

TheOREM 2.20. Let $(M,\langle\rangle$,$) be a manifold with a pole o.$

(i) ([Kum02]) Suppose that the radial sectional curvatures satisfy

$$
-1-\frac{\alpha}{r(x)} \leq K_{\mathrm{rad}}(x) \leq-1+\frac{\beta(1-\beta)}{r(x)} \quad \text { on } M \backslash\{o\} .
$$

For some $\alpha \geq 0, \beta \in[0,1]$ such that $2-(m-1) \alpha-(m+1) \beta>0$. Then, there exist no $L^{2}$ eigenfunctions related to eigenvalues $\lambda$ whenever

$$
\lambda \geq\left(\frac{(m-1)}{2-(m-1) \alpha-(m+1) \beta}\right)^{2} .
$$

(ii) ([DG97], Theorem 3.9 and [Esc85]) If $M=\left(M_{g}, \mathrm{~d} s^{2}\right)$ is a model with radial sectional curvature satisfying either

$$
\text { (i) } K_{\mathrm{rad}} \geq 0 \quad \text { or } \quad(\text { ii }) \quad K_{\mathrm{rad}} \leq 0 \quad \text { and } \quad K_{\mathrm{rad}}^{\prime} \geq 0 \text {, }
$$

then there exist no $L^{2}$ eigenfunctions related to positive eigenvalues.

REMARK 2.21. Observe that Theorem 2.20 and Corollary 2.17 jointly describe the whole spectrum of $\mathbb{R}^{m}$ and of hyperbolic space, $\mathbb{H}_{B}^{m}$, of sectional curvature $-B^{2}$.

REMARK 2.22. We mention that integral identities can be extended to analyze the operator $-\Delta$ on the space of $L^{2} p$-forms. For further insight, see $[\mathbf{R S 0 1}]$ and the references therein.

We conclude this section by giving an account of upper estimates for $\lambda_{1}^{-\Delta}(M)$ and inf $\sigma_{\text {ess }}(-\Delta)$, which have been deeply investigated by many authors since the '70s. In their pioneering work [CY75], S.Y. Cheng and S.T. Yau proved, among many other things, that a manifold $M$ with at most polynomial volume growth satisfies $\lambda_{1}^{\Delta}(M)=0$ ([CY75], Proposition 9). This is the case, for instance, of Euclidean space $\mathbb{R}^{m}$ and of any manifold of finite volume. A few years later, M.A. Pinsky [Pin81] turned his attention on Cartan-Hadamard manifolds, that is, simply connected manifolds of non-positive sectional curvature. He showed that if, for some $B \geq 0, K_{\mathrm{rad}} \rightarrow-B^{2}$ sufficiently fast, then $\lambda_{1}^{-\Delta}(M) \leq(m-1)^{2} B^{2} / 4$. His proof relies on comparison techniques for ODE in a way similar to that used in Corollary 2.17. A first important extension is due to M.E. Gage [Gag80] and H. Donnelly 
[Don81b], who weakened the conditions by only requiring the completeness of $M$ and

$$
\text { Ricc } \geq-(m-1) B^{2}\langle,\rangle \text {. }
$$

However, as shown by R. Brooks [Bro81] and M.E. Taylor [Tay89], sharp upper bounds for $\lambda_{1}^{-\Delta}(M)$ and $\inf \sigma_{\text {ess }}(-\Delta)$ can be obtained by only imposing growth condition on the volume of geodesic balls. Adapting Brooks technique, Y. Higuchi in [Hig01] extended his result by proving

Theorem 2.23. Let $(M,\langle\rangle$,$) be a complete Riemannian manifold. Then,$

(i) ([Bro81], [Tay89], [Hig01]). If $\operatorname{vol}(M)=+\infty$, then

$$
\inf \sigma_{\text {ess }}(-\Delta) \leq \frac{a^{2}}{4}, \quad \text { where } a=\liminf _{r \rightarrow+\infty} \frac{\log \operatorname{vol}\left(B_{r}\right)}{r} \text {. }
$$

(ii) $([$ Bro84], [Hig01]). If $\operatorname{vol}(M)<+\infty$, then

$$
\inf \sigma_{\text {ess }}(-\Delta) \leq \frac{a^{2}}{4}, \quad \text { where } \quad a=\liminf _{r \rightarrow+\infty}\left[-\frac{1}{r} \log \left(\operatorname{vol}(M)-\operatorname{vol}\left(B_{r}\right)\right)\right]
$$

Note that the results of Pinsky and Gage can be derived from this theorem and the volume comparison Theorem 1.25. Moreover, (2.40) and Persson formula (1.88) imply that

$$
\lambda_{1}^{-\Delta}\left(M \backslash B_{R}\right) \leq \frac{a^{2}}{4} \quad \text { for every } \quad R \geq 0 .
$$

In particular, if $\operatorname{vol}\left(B_{r}\right)$ is subexponential and $\operatorname{vol}(M)=+\infty$, then $\lambda_{1}^{-\Delta}(M)=$ $\inf \sigma_{\text {ess }}(-\Delta)=0$.

REMARK 2.24. On the contrary, if $\operatorname{vol}(M)<+\infty$ it is easy to construct manifolds where $0=\lambda_{1}^{-\Delta}(M)<\inf \sigma_{\text {ess }}(-\Delta)$, so that, by the min-max principle, the discrete spectrum is non-empty. For instance, we quote the following example of M.P. Do Carmo and D. Zhou [CZ99]. Let $M=\left(M_{g}, \mathrm{~d} s^{2}\right)$ be a model manifold whose defining function $g$ satisfies

$$
g(r)= \begin{cases}r & \text { if } r \in[0,1] \\ e^{-r} & \text { if } r \in[2,+\infty) .\end{cases}
$$

Then, $\operatorname{vol}(M)<+\infty$, hence $\lambda_{1}^{-\Delta}(M)=0$ by Cheng-Yau theorem. Furthermore, by Theorem 2.23, $\inf \sigma_{\text {ess }}(-\Delta) \leq(m-1)^{2} / 4$. We prove that equality holds. Indeed, for every $h \in \mathbb{R}$ the function $u(x)=e^{h r(x)}$ satisfies $\Delta u=\left(h^{2}-(m-1) h\right) u$ on $M \backslash B_{2}$. The minimum of the coefficient of $u$ in the RHS is attained when $h=(m-1) / 2$. In this case,

$$
\Delta u+\frac{(m-1)^{2}}{4} u=0 \quad \text { on } M \backslash B_{2} .
$$

applying a result of J. Barta [Bar37], extended to non-compact domains by Cheng and Yau [CY75] and H. Alencar and Do Carmo [AC93], we get

$$
\lambda_{1}^{-\Delta}\left(M \backslash B_{2}\right) \geq \sup _{\substack{u \in C^{2}\left(M \backslash B_{2}\right) \\ u>0}}\left[\inf _{M \backslash B_{2}}\left(\frac{-\Delta u}{u}\right)\right] \geq \frac{(m-1)^{2}}{4},
$$

so that, combining with Persson formula (1.88) and the above upper bound for $\inf \sigma_{\text {ess }}(-\Delta)$ we deduce inf $\sigma_{\text {ess }}(-\Delta)=(m-1)^{2} / 4$. 
It is interesting to see what happens if the volume growth of the manifold is faster than exponential, that is, if $\operatorname{vol}\left(\partial B_{r}\right) \asymp \exp \left\{a r^{\alpha}\right\}$ for some $\alpha>1$. In general, there exists no essential spectrum and one may ask what is the rate of growth of $\lambda_{1}^{-\Delta}\left(M \backslash B_{R}\right)$ as an increasing function of $R$. We will address this problem in Chapter 6, where we will also give a proof of (a weaker version of) Theorem 2.23. We observe that the bounds that we will obtain could have interesting applications, for instance, in estimating the volume growth of the Martin-Morales-Nadirashvili minimal surface, see the next section. In Chapter 4, we will recover some of the estimates from below with a different approach based on the critical curve of a manifold, that will be introduced in Section 3.2. As we will see, lower bounds will be the consequence of a non-Euclidean extension of the Hardy-Poincarè inequality

$$
\frac{(m-2)^{2}}{4} \int_{\mathbb{R}^{m}} \frac{u^{2}}{|x|^{2}} \leq \int_{\mathbb{R}^{m}}|\nabla u|^{2},
$$

where $m \geq 3$ and $u \in H^{1}\left(\mathbb{R}^{m}\right)$, usually called the uncertainty principle lemma. The link between the estimates in this section and those that we shall present reveals to be nontrivial, and will be subject of investigation.

\subsection{Spectral estimates and immersions}

The min-max characterization of eigenvalues, Persson formula (1.88) for the infimum of the essential spectrum, together with Barta inequality [Bar37] and its extensions ([CY75], [AC93], [BM07]), are particularly useful when $M$ is an isometrically immersed submanifold of some ambient space $N$. Next example, a mild generalization of a very recent result of G.P. Bessa, L.P. Jorge and J.F. Montenegro [BJM10], is instructive. In this paper, the authors addressed a question of S.T. Yau [Yau00]: is the spectrum of $-\Delta$ on the Nadirashvili minimal surface discrete? We recall that the Nadirashvili minimal surface, [Nad96], is the first example of a complete, minimal immersion in $\mathbb{R}^{3}$ with bounded image. Unfortunately, it is not known whether the Nadirashvili minimal surface is properly immersed or not (we recall that a map $\varphi: B \rightarrow D$ is proper if the pre-image of every compact subset of $D$ is compact in $B$ ); this is one of the reasons why the tricky construction via the Enneper-Weiestrass representation has been further refined by F. Martin and S. Morales in [MM05], [MM06]. In this way they exhibit, for every convex domain $D \subset \mathbb{R}^{3}$ a complete, proper, minimal immersion from the unit disk $B \subset \mathbb{C}$ into $D$. Martin-Morales highly nontrivial improvement on Nadirashvili construction is called in [BJM10] the Martin-Morales-Nadirashvili minimal surface. Note that both Nadirashvili and Martin-Morales-Nadirashvili examples, however, cannot be embeddings. In fact, embedded minimal surfaces of $\mathbb{R}^{3}$ must be unbounded, as showed by T. Colding and W. Minicozzi [CM05]. In their paper, Bessa, Jorge and Montenegro succeeded in proving that the spectrum of $-\Delta$ of the MartinMorales-Nadirashvili surface must be discrete ([BJM10], Theorem 1.2). As it will be apparent, the properness assumption is essential for their argument to work. Here we use their method to deal with a mildly more general situation. To state the theorem, we first need some definitions and preliminary computations.

Suppose that $\left(N^{n},\langle,\rangle_{N}\right),\left(Q^{q},\langle,\rangle_{Q}\right)$ are two complete Riemannian manifolds of dimension, respectively, $n$ and $q$, let $0<f \in C^{\infty}(N)$ and let $N \times_{f} Q$ be a warped product of $N$ and $Q$, that is, the product manifold $N \times Q$ with metric $\langle\langle\rangle\rangle=,\langle,\rangle_{N}+f^{2}\langle,\rangle_{Q}$. Denote with $\|\cdot\|$ the norm induced by $\langle\langle\rangle$,$\rangle on T\left(N \times_{f} Q\right)$, 
and with $\nabla$ the connection of $N$. Let $i_{N}, i_{Q}$ be the standard inclusions of $N$, respectively $Q$, into $N \times_{f} Q$, and let $\pi_{N}, \pi_{Q}$ be the projections of $N \times_{f} Q$ onto its components. We fix the index notation

$$
r, s, t \in\{1, \ldots, n\}, \quad \alpha, \beta, \gamma \in\{n+1, \ldots, n+q\}, \quad a, b, c \in\{1, \ldots, n+q\} .
$$

Consider a local frame $\left\{E_{r}\right\}$ in a neighbourhood of a point of $N$, its dual coframe $\left\{\omega^{s}\right\}$ and the connection forms $\left\{\omega_{s}^{r}\right\}$. Similarly, let $\left\{E_{\alpha}, \omega^{\beta}, \omega_{\beta}^{\alpha}\right\}$ locally describe the geometry of $Q$. Then, a local orthonormal coframe $\left\{\psi^{a}\right\}$ for $N \times_{f} Q$ is given by setting $\psi^{r}=\omega^{r}, \psi^{\alpha}=f \omega^{\alpha}$. Accordingly, the dual frame $\left\{\xi_{a}\right\}$ is given by $\xi_{r}=E_{r}$, $\xi_{\alpha}=E_{\alpha} / f$. An inspection of the structure equations of $N, Q$ and $N \times_{f} Q$ shows that the connection forms $\left\{\psi_{b}^{a}\right\}$ of $N \times_{f} Q$ are given by

$$
\psi_{s}^{r}=\omega_{s}^{r}, \quad \psi_{\beta}^{\alpha}=\omega_{\beta}^{\alpha}, \quad \psi_{r}^{\alpha}=f_{r} \omega^{\alpha}=\frac{f_{r}}{f} \psi^{\alpha},
$$

where $\mathrm{d} f=f_{r} \omega^{r}$. For future use, we need to compute the Hessian of a smooth function $h$ on $M \times{ }_{f} Q$. Let $\left\{h_{a b}\right\}$ be the components of Hess $h$ in the basis $\left\{\psi^{a} \otimes \psi^{b}\right\}$. Towards this purpose, let $\mathrm{d} h=h_{r} \psi^{r}+h_{\alpha} \psi^{\alpha}$. We agree to denote with a subscript $N$, respectively $Q$, the projection of $T\left(N \times_{f} Q\right)$ onto the subbundles generated by $\left\{\xi_{r}\right\}$, respectively $\left\{\xi_{\alpha}\right\}$, so that, for instance,

$$
\mathrm{d}_{N} h=h_{r} \psi^{r} \equiv \mathrm{d}\left(h \circ i_{N}\right), \quad \mathrm{d}_{Q} h=h_{\alpha} \psi^{\alpha} \equiv \mathrm{d}\left(h \circ i_{Q}\right),
$$

where the equivalences hold up to obvious identifications. Decomposing the expression for the covariant derivative

$$
h_{r a} \psi^{a}=\mathrm{d} h_{r}-h_{s} \psi_{r}^{s}-h_{\alpha} \psi_{r}^{\alpha}, \quad h_{\beta a} \psi^{a}=\mathrm{d} h_{\beta}-h_{s} \psi_{\beta}^{s}-h_{\alpha} \psi_{\beta}^{\alpha}
$$

along the basis $\psi^{r}, \psi^{\alpha}$, and using (2.42) we get

$$
\begin{cases}(i) & h_{r s}=\xi_{s}\left(h_{r}\right)-h_{t} \omega_{r}^{t}\left(\xi_{s}\right)=E_{s} E_{r}(h)-E_{t}(h) \omega_{r}^{t}\left(E_{s}\right)=\left({ }^{N} \operatorname{Hess} h\right)_{r s} \\ (\text { ii }) & h_{r \alpha}=\xi_{\alpha}\left(h_{r}\right)-\frac{h_{\alpha} f_{r}}{f}=\xi_{r}\left(h_{\alpha}\right)=h_{\alpha r} \\ (\text { iii }) & h_{\alpha_{\beta}}=\frac{h_{s} f_{s}}{f} \delta_{\alpha \beta}+\xi_{\beta}\left(h_{\alpha}\right)-h_{\gamma} \omega_{\alpha}^{\gamma}\left(\xi_{\beta}\right),\end{cases}
$$

where ${ }^{N}$ Hess $h$ is the Hessian of the function $h \circ i_{N} \in C^{\infty}(N)$. In order to make the Hessian of $h \circ i_{Q}$ to appear in the third equation, we write $\mathrm{d}\left(h \circ i_{Q}\right)=\bar{h}_{\alpha} \omega^{\alpha}$. From $\mathrm{d}\left(h \circ i_{Q}\right)=\mathrm{d}_{Q} h=h_{\alpha} \psi^{\alpha}$ we deduce $\bar{h}_{\alpha}=f h_{\alpha}$. The coefficients of ${ }^{Q} \operatorname{Hess} h$ in the basis $\omega^{\alpha} \otimes \omega^{\beta}$ are given by the expression

$$
\left({ }^{Q} \operatorname{Hess} h\right)_{\alpha \beta}=E_{\beta}\left(\bar{h}_{\alpha}\right)-\bar{h}_{\gamma} \omega_{\alpha}^{\gamma}\left(E_{\beta}\right)
$$

Taking into account that $E_{\alpha}(f)=0$ for every $\alpha$, we can rewrite (2.43), (iii) as

$$
h_{\alpha_{\beta}}=\frac{h_{s} f_{s}}{f} \delta_{\alpha \beta}+\frac{E_{\beta}}{f}\left(\frac{\bar{h}_{\alpha}}{f}\right)-\frac{\bar{h}_{\gamma}}{f} \omega_{\alpha}^{\gamma}\left(\frac{E_{\beta}}{f}\right)=\frac{h_{s} f_{s}}{f} \delta_{\alpha \beta}+\frac{1}{f^{2}}\left({ }^{Q} \operatorname{Hess} h\right)_{\alpha \beta}
$$

Let now $\varphi: M^{m} \rightarrow N \times_{f} Q$ be a smooth map, and define $u=h \circ \varphi$. Our next task is to compute the Hessian of $u$. With the index convention $i, j, k \in\{1, \ldots, m\}$, let $\left\{e_{i}, \theta^{j}, \theta_{j}^{i}\right\}$ be a local description of the geometry of $M$. Then, the differential $\mathrm{d} \varphi$, its Hilbert-Schmidt norm $\|\mathrm{d} \varphi\|^{2}$, the generalized second fundamental form $\nabla \mathrm{d} \varphi$ 
and the tension field $\tau(\varphi)$ are given by

$$
\left\{\begin{array}{l}
\mathrm{d} \varphi=\varphi_{i}^{a} \theta^{i} \otimes \xi_{a}, \quad\|\mathrm{~d} \varphi\|^{2}=\varphi_{i}^{a} \varphi_{i}^{a}, \\
\nabla \mathrm{d} \varphi=\varphi_{i j}^{a} \theta^{j} \otimes \theta^{i} \otimes \xi_{a}, \quad \text { where } \\
\varphi_{i j}^{a} \theta^{j}=\mathrm{d} \varphi_{i}^{a}-\varphi_{j}^{a} \theta_{i}^{j}+\varphi_{i}^{b} \psi_{b}^{a}, \\
\tau(\varphi)=\varphi_{i i}^{a} \xi_{a} .
\end{array}\right.
$$

From the chain rule, we have

$$
\text { Hess } u=\operatorname{Hess} h(\mathrm{~d} \varphi \otimes \mathrm{d} \varphi)+\mathrm{d} h \circ \nabla \mathrm{d} \varphi,
$$

hence, taking traces,

$$
\Delta u=\sum_{i} \operatorname{Hess} h\left(\mathrm{~d} \varphi\left(e_{i}\right), \mathrm{d} \varphi\left(e_{i}\right)\right)+\mathrm{d} h(\tau(\varphi))
$$

Suppose now that $h$ is a function that only depends on the points of $N$, so that the mixed terms $h_{r \alpha}$ vanish. Then, the first term in the RHS of (2.44) can be written as

$$
h_{r s} \varphi_{i}^{r} \varphi_{i}^{s}+h_{\alpha \beta} \varphi_{i}^{\alpha} \varphi_{i}^{\beta}=\left({ }^{N} \operatorname{Hess} h\right)_{r s} \varphi_{i}^{s} \varphi_{i}^{r}+\frac{h_{s} f_{s}}{f} \varphi_{i}^{\alpha} \varphi_{i}^{\alpha} .
$$

Consequently, we can rewrite (2.44) as follows:

$$
\Delta u=\sum_{i}\left({ }^{N} \operatorname{Hess} h\right)\left(\mathrm{d}_{N} \varphi\left(e_{i}\right), \mathrm{d}_{N} \varphi\left(e_{i}\right)\right)+\langle\nabla h, \nabla \log f\rangle_{N}\left\|\mathrm{~d}_{Q} \varphi\right\|^{2}+\left\langle\nabla h, \tau_{N}(\varphi)\right\rangle
$$

Next, let $k \in \mathbb{R}$, and let $\mathrm{sn}_{k}$ be the solution of the Cauchy problem

$$
\left\{\begin{array}{l}
\mathrm{sn}_{k}^{\prime \prime}+k \mathrm{sn}_{k}=0 \\
\mathrm{sn}_{k}(0)=0, \quad \operatorname{sn}_{k}^{\prime}(0)=1
\end{array}\right.
$$

that is,

$$
\operatorname{sn}_{k}(r)= \begin{cases}\sin (\sqrt{k} r) / \sqrt{k} & \text { if } k>0, \\ r & \text { if } k=0, \\ \sinh (\sqrt{-k} r) / \sqrt{-k} & \text { if } k<0 .\end{cases}
$$

Define $\mathrm{cn}_{k}(r)=\operatorname{sn}_{k}^{\prime}(r)$. We are ready to state

TheOREM 2.25. Let $N \times{ }_{f} Q$ be a warped product as above. Let $\rho(x)$ be the distance function on $N$ from a reference origin $p$, and let $B_{R_{0}} \subset N$ be a geodesic ball centered at $p$ of radius $R_{0}$. Define $k \in \mathbb{R}$ to be an upper bound of the radial sectional curvatures at points of $B_{R_{0}}$. If $k>0$, we restrict to the case $R_{0}<\pi /(2 \sqrt{k})$. Let $M^{m}$ be a non-compact Riemannian manifold, possibly non complete, and let $\varphi: M \rightarrow N^{n} \times{ }_{f} Q^{q}$ be a smooth map whose image lies in the cylindrical region $B_{R_{0}} \times Q$. Assume that the following properties hold:

(i) $\varphi^{-1}\left(B_{R} \times Q\right)$ is relatively compact for every $R<R_{0}$;

(ii) $\quad \liminf _{x \rightarrow \infty}\left\|\mathrm{d}_{N} \varphi(x)\right\|^{2} \geq A>0, \quad \limsup _{x \rightarrow \infty}\left\|\mathrm{d}_{Q} \varphi(x)\right\|^{2} \leq B<+\infty$.

If

$$
\limsup _{x \rightarrow \infty}\|\tau(\varphi)(x)\|<A \frac{\mathrm{cn}_{k}\left(R_{0}\right)}{\operatorname{sn}_{k}\left(R_{0}\right)}-B\||\nabla \log f|\|_{C^{0}\left(\partial B_{R_{0}}\right)},
$$

then $-\Delta$ on $M$ has only discrete spectrum. 
Proof. For $R \in\left(0, R_{0}\right)$, let $\Omega_{R}=\varphi^{-1}\left(B_{R} \times Q\right)$. By $(i),\left\{\Omega_{R}\right\}$ is an exhaustion of $M$ by relatively compact domains. Let $j_{0} \in \mathbb{N}$ be sufficiently large and, for every $j \geq j_{0}$, let $R_{j}, \Omega_{j}$ be such that

$$
\left\|\mathrm{d}_{N} \varphi\right\|^{2} \geq A_{j}=A-\frac{1}{j}>0, \quad\left\|\mathrm{~d}_{Q} \varphi\right\|^{2} \leq B_{j}=B+\frac{1}{j} \quad \text { on } \quad M \backslash \Omega_{R_{j}}=M \backslash \Omega_{j}
$$

Clearly, we can assume $R_{j} \uparrow R_{0}$. Set also $D_{j}=B_{R_{0}} \backslash B_{R_{j}}$. Define, for $r \in\left(0, R_{0}\right)$

$$
\operatorname{in}_{k}(r)=\int_{r}^{R_{0}} \operatorname{sn}_{k}(s) \mathrm{d} s
$$

and consider the function $h: N \times_{f} Q \rightarrow \mathbb{R}$ given by $h(x, y)=\operatorname{in}_{k}(\rho(x))$. From the Hessian comparison Theorem 1.15, and since $\mathrm{in}_{k}$ is decreasing, we deduce

$$
\begin{aligned}
{ }^{N} \operatorname{Hess} h & =\operatorname{in}_{k}^{\prime \prime}(\mathrm{d} \rho \otimes \mathrm{d} \rho)+\operatorname{in}_{k}^{\prime}\left({ }^{N} \operatorname{Hess} \rho\right) \\
& \leq-\operatorname{cn}(\mathrm{d} \rho \otimes \mathrm{d} \rho)-\operatorname{sn}_{k} \frac{\operatorname{cn}_{k}}{\operatorname{sn}_{k}}\left(\langle,\rangle_{N}-\mathrm{d} \rho \otimes \mathrm{d} \rho\right)=-\operatorname{cn}_{k}\langle,\rangle_{N} .
\end{aligned}
$$

By formula (2.45), the Laplacian of $u=h \circ \varphi$ is bounded as follows:

$$
\begin{aligned}
\Delta u & \leq-\operatorname{cn}_{k}\left\|\mathrm{~d}_{N} \varphi\right\|^{2}+\operatorname{sn}_{k}|\nabla \log f|\left\|\mathrm{d}_{Q} \varphi\right\|^{2}+\operatorname{sn}_{k}\|\tau(\varphi)\| \\
& =-\operatorname{sn}_{k}\left(\frac{\mathrm{cn}_{k}}{\operatorname{sn}_{k}}\left\|\mathrm{~d}_{N} \varphi\right\|^{2}+|\nabla \log f|\left\|\mathrm{d}_{Q} \varphi\right\|^{2}+\|\tau(\varphi)\|\right)
\end{aligned}
$$

By (2.47) and by (2.48), if $j$ is sufficiently large and $x \in M \backslash \Omega_{j}$ we get

$$
\frac{\mathrm{cn}_{k}\left(R_{0}\right)}{\operatorname{sn}_{k}\left(R_{0}\right)} A_{j}-\||\nabla \log f|\|_{C^{0}\left(D_{j}\right)} B_{j}-\|\tau(\varphi)\| \geq c,
$$

for some $c>0$ independent of $j$. Therefore, since $\mathrm{cn}_{k} / \mathrm{sn}_{k}$ is decreasing (on $(0, \pi /(2 \sqrt{k}))$, if $k>0),(2.49)$ implies

$$
\Delta u \leq-c \cdot \operatorname{sn}_{k} \leq-c \cdot \operatorname{sn}_{k}\left(R_{j}\right)
$$

Therefore, an application of Barta inequality, together with (2.51) gives

$$
\lambda_{1}^{-\Delta}\left(M \backslash \Omega_{j}\right) \geq \inf _{M \backslash \Omega_{j}}\left(-\frac{\Delta u}{u}\right) \geq c \frac{\operatorname{sn}_{k}\left(R_{j}\right)}{\operatorname{in}_{k}\left(R_{j}\right)},
$$

and letting $j \rightarrow+\infty$ with the aid of Persson formula (1.88) we deduce

$$
\inf \sigma_{\mathrm{ess}}(-\Delta) \geq \lim _{j \rightarrow+\infty} c \frac{\mathrm{sn}_{k}\left(R_{j}\right)}{\operatorname{in}_{k}\left(R_{j}\right)}=+\infty
$$

By the min-max characterization, $-\Delta$ has only discrete spectrum.

REMARK 2.26. We observe that, loosely speaking, property $(i)$ in (2.46) requires that $\varphi(x)$ tends to the boundary of the cylinder uniformly as $x$ diverges in $M$.

The next corollaries are immediate consequences of the above theorem. We first consider the particular case when $\varphi$ is an isometric immersion. As usual, we denote with $I I$ the second fundamental form $\nabla \mathrm{d} \varphi$, and with $H$ the mean curvature vector, normalized according to $m H=\tau(\varphi)=\operatorname{Tr}(I I)$. 
Corollary 2.27 ([BJM10], Theorem 4.1). In the assumptions of the above theorem, let

$$
\varphi: M^{m} \rightarrow B_{R_{0}} \times_{f} Q^{q} \subset N^{n} \times_{f} Q^{q}
$$

be an isometric immersion satisfying property $(i)$ of (2.46), and assume that $m>q$. If

$$
\limsup _{x \rightarrow \infty}\|H(x)\|<\frac{(m-q)}{m} \frac{\mathrm{cn}_{k}\left(R_{0}\right)}{\operatorname{sn}_{k}\left(R_{0}\right)}-\frac{q}{m}\||\nabla \log f|\|_{C^{0}\left(\partial B_{R_{0}}\right)},
$$

then $-\Delta$ on $M$ has only discrete spectrum. In particular, if $\varphi$ is minimal and $f_{\mid \partial B_{R_{0}}}$ satisfies

$$
\||\nabla \log f|\|_{C^{0}\left(\partial B_{R_{0}}\right)}<\frac{(m-q)}{q} \frac{\mathrm{cn}_{k}\left(R_{0}\right)}{\operatorname{sn}_{k}\left(R_{0}\right)},
$$

then, the spectrum of $-\Delta$ on $M$ is discrete.

Proof. We only prove the first part of the statement, the second being an immediate consequence. Since $\varphi$ is isometric, $\left\{\mathrm{d} \varphi\left(e_{i}\right)\right\}$ is an orthonormal set, hence $\left\|\mathrm{d}_{N} \varphi\right\|^{2}+\left\|\mathrm{d}_{Q} \varphi\right\|^{2}=\|\mathrm{d} \varphi\|^{2}=m$,

$$
\left\|\mathrm{d}_{Q} \varphi\right\|^{2}=\varphi_{i}^{\alpha} \varphi_{i}^{\alpha}=\sum_{\alpha}\left(\sum_{i}\left\langle\left\langle\mathrm{~d} \varphi\left(e_{i}\right), E_{\alpha}\right\rangle\right\rangle\right) \leq \sum_{\alpha} 1=q,
$$

and thus $\left\|\mathrm{d}_{N} \varphi\right\|^{2} \geq m-q$. Inserting $(m-q)$ and $q$ in place of $A, B$ in (2.47) we reach the desired conclusion.

Corollary 2.28. Let $M$ be a Riemannian manifold such that there exists a proper harmonic map $\varphi$ into some relatively compact ball $B_{R_{0}} \subset N^{n}$. Denote with $k$ an upper bound for the radial sectional curvatures of points of $B_{R_{0}}$. If $k>0$, assume furthermore that $R_{0}<\pi /(2 \sqrt{k})$. Then, if $\|\mathrm{d} \varphi\|^{2} \geq C>0$ outside some compact set, the spectrum of $-\Delta$ on $M$ is discrete.

Proof. Roughly speaking, it is enough to get rid of $Q$ and $f$ in Theorem 2.25. Indeed, the computations and the steps of the proof can be straightforwardly rephrased in this slightly different setting, and by the harmonicity assumption $\tau(\varphi)=0$ the conclusion follows easily.

As a particular case of Corollary 2.28, we recover

Corollary 2.29 ([BJM10], Theorem 1.2). The Martin-Morales-Nadirashvili minimal surface has discrete spectrum.

\subsection{Spectral estimates and nonlinear PDE}

Spectral theory is intimately related to existence and non-existence results for semilinear elliptic equations. To justify this claim, we consider as a prototype example the classical Yamabe problem. Let $(M,\langle\rangle$,$) be a Riemannian manifold$ with dimension $m \geq 3$, volume form $\mathrm{d} V$ and scalar curvature $s$, and let

$$
\widetilde{\langle,\rangle}=u^{\frac{4}{m-2}}\langle,\rangle, \quad 0<u \in C^{\infty}(M)
$$


be a (pointwise) conformal deformation of the metric. Adding a tilde to geometric quantities referring to $(M, \widetilde{\langle,\rangle}), \mathrm{d} \widetilde{V}=u^{2 m /(m-2)} \mathrm{d} V$. A computation shows that the scalar curvatures are related by Yamabe equation

$$
\Delta u-\frac{s(x)}{c_{m}} u+\frac{\widetilde{s}(x)}{c_{m}} u^{\frac{m+2}{m-2}}=0, \quad \text { where } \quad c_{m}=\frac{4(m-1)}{m-2}
$$

(see $[\mathbf{K a z 8 5}]$ ). The existence of a conformal deformation of the metric with assigned scalar curvature $\widetilde{s}$ is equivalent to the solvability of (2.55) with $u>0$. Set $L=$ $-\Delta+s / c_{m}, \widetilde{L}=-\widetilde{\Delta}+\widetilde{s} / c_{m} . L$ is usually called the conformal Laplacian of $M$. From the transformation law for $\Delta$ under a conformal change of the metric, that is,

$$
\widetilde{\Delta} \phi=u^{-\frac{4}{m-2}} \Delta \phi+2 u^{-\frac{m+2}{m-2}}\langle\nabla u, \nabla \phi\rangle \quad \forall \phi \in C^{2}(M),
$$

the following relations hold for every $\phi \in \operatorname{Lip}_{c}(M)$ (respectively, $\phi \in C^{2}(M)$ ):

$$
\begin{gathered}
\int_{M}|\widetilde{\widetilde{\nabla}} \phi|^{2} \mathrm{~d} \widetilde{V}+\int_{M} \frac{\widetilde{s}}{c_{m}} \phi^{2} \mathrm{~d} \widetilde{V}=\int_{M}|\nabla(u \phi)|^{2} \mathrm{~d} V-\int_{M} \frac{s}{c_{m}}(u \phi)^{2} \mathrm{~d} V, \\
\widetilde{L} \phi=u^{-\frac{m+2}{m-2}} L(u \phi) .
\end{gathered}
$$

From this and the variational characterization (1.78), the signs of $\lambda_{k}^{L}(\Omega)$ and $\widetilde{\lambda}_{k}^{\widetilde{L}}(\Omega)$ coincide for every $\Omega \Subset M$. Spectral assumptions on $L$ such as stability, either global or outside a compact set, are thus conformal invariants. As a consequence, it is expected that the sign of $\lambda_{1}^{L}(M)$, for instance, be relevant for existence or nonexistence of positive solutions $u$ of $(2.55)$. This is indeed true for a wider class of nonlinearities. As an example we consider the following theorem, which combines the method of sub-supersolutions as described in [A085], [AO88] with ideas in [BRS98a], [RRV94], [RRV97]. This has been further extended in [PRS10] to the present.

TheOREM 2.30. Let $(M,\langle\rangle$,$) be a non-compact Riemannian manifold of di-$ mension $m \geq 2$, and let $q(x), b(x) \in C_{\mathrm{loc}}^{0, \mu}(M), \mu \in(0,1]$. Let $b(x) \geq 0$ on $M$ and strictly positive outside a compact set. Having set

$$
B_{0}=\{x \in M: b(x)=0\},
$$

assume that $\lambda_{1}^{L}\left(B_{0}\right)>0$, where $L=-\Delta-q(x)$. Suppose furthermore that

$$
\lambda_{1}^{L}(M)<0 .
$$

Then, for every $\sigma>1$, the equation

$$
\Delta u+q(x) u-b(x) u^{\sigma}=0
$$

possesses a minimal and a maximal (possibly coinciding) positive $C_{\mathrm{loc}}^{2, \mu}$ solutions.

REMARK 2.31. Since the first eigenvalue of $-\Delta$ on $B_{r}$ grows like $r^{-2}$ as $r \rightarrow 0$, for each $q(x) \in L_{\text {loc }}^{\infty}(M)$ we have $\lambda_{1}^{L}\left(B_{r}\right)>0$ provided $r$ is sufficiently small. One may therefore think that the condition $\lambda_{1}^{L}\left(B_{0}\right)>0$ expresses the fact that $B_{0}$ is "small", at least in a spectral sense.

For the convenience of the reader, we divide the proof into several steps. The first is a simple comparison. 
Proposition 2.32. Let $\Omega \subset M$ be a bounded domain with Lipschitz boundary. Assume that $q(x), b(x) \in C^{0}(\bar{\Omega})$ and that $b(x) \geq 0$. Let $u, v \in C^{2}(\Omega) \cap C^{0}(\bar{\Omega})$ be solutions on $\Omega$ of

$$
\begin{aligned}
& \Delta u+q(x) u-b(x) u^{\sigma} \leq 0 ; \\
& \Delta v+q(x) v-b(x) v^{\sigma} \geq 0 .
\end{aligned}
$$

With $v \geq 0, u>0$ and $\sigma \geq 1$. If $v \leq u$ on $\partial \Omega$ then $v \leq u$ on $\Omega$.

Proof. The proof is modelled on that of the generalized maximum principle, see [PW84]. Set $w=v / u$. A computation using (2.58) shows

$$
\Delta w \geq b(x)\left(v^{\sigma-1}-u^{\sigma-1}\right) w-2\langle\nabla w, \nabla \log v\rangle .
$$

If, by contradiction, $v>u$ somewhere on $\Omega$, let $\varepsilon$ be sufficiently small that

$$
\Omega_{\varepsilon}=\{x \in \Omega: w(x)>1+\varepsilon\} \neq \emptyset .
$$

Since $v \geq u$ and $b(x) \geq 0$ on $\Omega_{\varepsilon}$,

$$
\Delta w+2\langle\nabla w, \nabla \log v\rangle \geq 0 \quad \text { on } \Omega_{\varepsilon} .
$$

From $w=1+\varepsilon$ on $\partial \Omega_{\varepsilon}$, applying the maximum principle we deduce $w \leq 1+\varepsilon$ on $\Omega_{\varepsilon}$, contradicting $\Omega_{\varepsilon} \neq \emptyset$.

Next, we state and prove a mild improvement of an original result of P. Li, L.F. Tam and D. Yang [LTD98].

Proposition 2.33. Let $q(x), b(x) \in C_{\mathrm{loc}}^{0, \mu}(M), \mu \in(0,1], b(x) \geq 0$ and suppose that $B_{0}$ is compact. Let $\Omega$ be a relatively compact open domain with smooth boundary containing $B_{0}$. If

$$
\Delta u+q(x) u-b(x) u^{\sigma}=0, \quad \sigma>1
$$

has a positive weak supersolution $u \in H^{1}(\Omega) \cap L_{\mathrm{loc}}^{\infty}(\Omega)$, then $\lambda_{1}^{L}\left(B_{0}\right) \geq 0$. Conversely, if $\lambda_{1}^{L}\left(B_{0}\right)>0$, then (2.59) has a positive supersolution $u \in C^{2, \mu}(\bar{\Omega})$.

Proof. Suppose $u \in H^{1}(\Omega) \cap L_{\text {loc }}^{\infty}(\Omega)$ is a positive weak supersolution of (2.59) on $\Omega$ and, by contradiction, assume that $\lambda_{1}^{L}\left(B_{0}\right)=-a$, for some $a>0$. Then, by the definition of $\lambda_{1}^{L}\left(B_{0}\right)$ we can find a sequence of open sets with smooth boundaries $\Omega_{i}, i \in \mathbb{N}$, such that

$$
\Omega_{i+1} \Subset \Omega_{i} \Subset \Omega, \quad \bigcap_{i=1}^{+\infty} \Omega_{i}=B_{0},
$$

and, increasingly, $\lambda_{i}=\lambda_{1}^{L}\left(\Omega_{i}\right) \rightarrow-a$ as $i \rightarrow+\infty$. Corresponding to $\lambda_{i}$, there exists a $C^{2, \mu}$ positive eigenfunction $v_{i}$ such that

$$
\begin{cases}\Delta v_{i}+q(x) v_{i}=-\lambda_{i} v_{i} & \text { on } \Omega_{i} \\ v_{i}>0 \quad \text { on } \Omega_{i}, \quad v_{i}=0 & \text { on } \partial \Omega_{i} .\end{cases}
$$

Note that, since $v_{i}>0$, on $\Omega_{i}$,

$$
\frac{\partial v_{i}}{\partial \nu} \leq 0
$$

$\nu$ being the outward pointing unit normal to $\partial \Omega_{i}$. Using Green formula and the fact that $u>0$ solves $\Delta u+q(x) u \leq b(x) u^{\sigma}$ weakly on $\Omega_{i}$ we get

$$
0 \geq \int_{\partial \Omega_{i}} u \frac{\partial v_{i}}{\partial \nu}=\int_{\Omega_{i}} u \Delta v_{i}+\int_{\Omega_{i}}\left\langle\nabla v_{i}, \nabla u\right\rangle \geq \int_{\Omega_{i}}-v_{i} u\left(\lambda_{i}+b(x) u^{\sigma-1}\right),
$$


that is,

$$
\int_{\Omega_{i}} u v_{i}\left(\lambda_{i}+b(x) u^{\sigma-1}\right) \geq 0 .
$$

Since $\Omega_{i} \downarrow B_{0}$ and $u \in L_{\mathrm{loc}}^{\infty}(\Omega)$, using both the continuity of $b(x)$ and $\lambda_{i} \rightarrow-a<0$ for $i$ sufficiently large we contradict (2.62).

To prove the converse, assume $\lambda_{1}^{L}\left(B_{0}\right)>0$. Let $\Lambda, \Lambda^{\prime}$ be open sets with smooth boundary such that

$$
B_{0} \subset \Lambda^{\prime} \Subset \Lambda \Subset \Omega \quad \text { and } \quad \lambda_{1}^{L}(\Lambda)>0 .
$$

Let $u_{1}$ be a solution of

$$
\begin{cases}\Delta u_{1}+q(x) u_{1}=-\lambda_{1}^{L}(\Lambda) u_{1} & \text { on } \Lambda, \\ u_{1}=0 & \text { on } \partial \Lambda .\end{cases}
$$

By elliptic regularity up to the boundary, $u_{1} \in C^{2, \mu}(\bar{\Lambda})$ ([GT98], Theorem 6.6) and, by Remark $1.28, u_{1}>0$ on $\Lambda$. Since $b(x)>0$ on $\bar{\Omega} \backslash \Lambda^{\prime}$, we can define

$$
\beta=\inf _{\Omega \backslash \Lambda^{\prime}} b>0 .
$$

We claim that a sufficiently large positive constant $u_{2}$ is a supersolution of (2.59) on $\Omega \backslash \Lambda^{\prime}$. Towards this aim we let

$$
A=\sup _{\Omega \backslash \Lambda^{\prime}} q .
$$

Note that $A<+\infty$ since $\Omega$ has compact closure. Then we have

$$
\Delta u_{2}+q(x) u_{2}-b(x) u_{2}^{\sigma}=u_{2}\left[q(x)-b(x) u_{2}^{\sigma-1}\right] \leq u_{2}\left[A-\beta u_{2}^{\sigma-1}\right] \leq 0
$$

provided $u_{2} \geq(A / \beta)^{1 /(\sigma-1)}$. Let now $\psi \in C_{c}^{\infty}(\Lambda)$ be a smooth cut-off function such that $0 \leq \psi \leq 1, \psi \equiv 1$ on $\Lambda^{\prime}$. Fix a positive constant $\gamma$ and define

$$
u=\gamma\left(\psi u_{1}+(1-\psi) u_{2}\right) \quad \in C^{2, \mu}(\bar{\Omega})
$$

Then, on $\Lambda^{\prime}$, where $u \equiv \gamma u_{1}$, we have

$$
\Delta u+q(x) u-b(x) u^{\sigma}=-\left[\lambda_{1}^{L}(\Lambda)+b(x)\left(\gamma u_{1}\right)^{\sigma-1}\right] \gamma u_{1} \leq 0
$$

irrespectively of the value of $\gamma>0$. Moreover, on $\Omega \backslash \Lambda$, where $u \equiv \gamma u_{2}$, we get

$$
\Delta u+q(x) u-b(x) u^{\sigma}=\gamma\left[q(x) u_{2}-b(x) u_{2}^{\sigma} \gamma^{\sigma-1}\right] .
$$

Now, for $\gamma \geq 1$ and since $b>0$ on $\Omega \backslash \Lambda$, we deduce $b(x) \gamma^{\sigma} \geq b(x) \gamma$, so that

$$
\Delta u+q(x) u-b(x) u^{\sigma} \leq \gamma\left[\Delta u_{2}+q(x) u_{2}-b(x) u_{2}^{\sigma}\right] \leq 0
$$

because of our choice of $u_{2}$. It remains to analyze the situation on $\Lambda \backslash \Lambda^{\prime}$. On this set

$$
(\Delta+q(x))\left(\psi u_{1}+(1-\psi) u_{2}\right) \leq C,
$$

for some $C>0$ sufficiently large. Now, since $b(x)>0$ on $\overline{\Lambda \backslash \Lambda^{\prime}}$,

$$
\inf _{\Lambda \backslash \Lambda^{\prime}} b(x)\left(\psi u_{1}+(1-\psi) u_{2}\right)^{\sigma}>C^{-1}
$$


up to enlarging $C$ further. Therefore, on $\Lambda \backslash \Lambda^{\prime}$ we have

$$
\begin{aligned}
\Delta u+q(x) u-b(x) u^{\sigma}= & \gamma(\Delta+q(x))\left(\psi u_{1}+(1-\psi) u_{2}\right) \\
& -b(x) \gamma^{\sigma}\left(\psi u_{1}+(1-\psi) u_{2}\right)^{\sigma} \\
\leq & \gamma\left(C-\gamma^{\sigma-1} C^{-1}\right) \leq 0
\end{aligned}
$$

up to choosing $\gamma \geq C^{2 /(\sigma-1)}$. Thus, $u$ is a supersolution on $\Omega$ whenever $\gamma \geq$ $\max \left\{1, C^{2 /(\sigma-1)}\right\}$.

Next, we proceed to construct solutions on relatively compact domains.

Lemma 2.34. Let $q(x), b(x) \in C_{\mathrm{loc}}^{0, \mu}(M), \mu \in(0,1], b(x) \geq 0$ and suppose that $B_{0}$ is compact and satisfies $\lambda_{1}^{L}\left(B_{0}\right)>0$. Let $\Omega$ be a relatively compact open domain with smooth boundary such that $B_{0} \Subset \Omega$. Fix $n \in(0,+\infty)$. Then, there exists $u \in C^{2, \mu}(\bar{\Omega})$ which solves the problem

$$
\left\{\begin{array}{l}
\Delta u+q(x) u-b(x) u^{\sigma}=0 \quad \text { on } \Omega, \\
u>0 \quad \text { on } \Omega, \quad u=n \quad \text { on } \partial \Omega .
\end{array}\right.
$$

Proof. By the definition of $\lambda_{1}^{L}\left(B_{0}\right)$, there exists an open domain with smooth boundary $D$ such that $B_{0} \Subset D \Subset \Omega$ and $\lambda_{1}^{L}(D)>0$. Let $\psi \in C_{c}^{\infty}(\Omega)$ be a cut-off function such that $0 \leq \psi \leq 1$ and $\psi \equiv 1$ on $D$. Fix

$$
N \geq \max \left\{1+\sup _{\bar{\Omega}}|q(x)|, \lambda_{1}^{-\Delta}(M)+1\right\}
$$

and define

$$
\hat{q}(x)=\psi(x) q(x)+N(1-\psi(x)) \quad \in C^{2, \mu}(\bar{\Omega}) .
$$

Consider the operator $\hat{L}=-\Delta-\hat{q}(x)$. Since $\hat{q}=q$ on $D$, we have $\lambda_{1}^{\hat{L}}\left(B_{0}\right) \equiv$ $\lambda_{1}^{L}\left(B_{0}\right)>0$. Furthermore, since $N \geq \lambda_{1}^{-\Delta}(M)+1$, there exists a sufficiently large, relatively compact domain $\Omega_{1}$ such that

$$
\bar{\Omega} \subset \Omega_{1} \quad \text { and } \quad \lambda_{1}^{\hat{L}}\left(\Omega_{1}\right)<0 .
$$

We fix $\gamma>0$ sufficiently small in such a way that, if $\varphi \in C^{2, \mu}\left(\bar{\Omega}_{1}\right)$ is a normalized eigenfunction of $\hat{L}$ on $\Omega_{1}$, that is, if $\varphi$ satisfies

$$
\begin{cases}\hat{L} \varphi=\lambda_{1}^{\hat{L}}\left(\Omega_{1}\right) & \text { on } \Omega_{1} \\ \varphi=0 & \text { on } \partial \Omega_{1}\end{cases}
$$

and $\|\varphi\|_{L^{2}\left(\Omega_{1}\right)}=1$, then

$$
\int_{\Omega_{1}}\left[|\nabla \varphi|^{2}-\hat{q}(x) \varphi^{2}\right]+\gamma \int_{\Omega_{1}} b(x) \varphi^{2}=\lambda_{1}^{\hat{L}}\left(\Omega_{1}\right)+\gamma \int_{\Omega_{1}} b(x) \varphi^{2}<0 .
$$

This shows that the operator $\widetilde{L}=\hat{L}+\gamma b(x)$ satisfies $\lambda_{1}^{\widetilde{L}}\left(\Omega_{1}\right)<0$. Let $\psi \in C^{2, \mu}\left(\bar{\Omega}_{1}\right)$ be an eigenfunction corresponding to $\lambda_{1}^{\widetilde{L}}\left(\Omega_{1}\right)$. Then, $\psi$ is positive by Remark 1.28 , and satisfies

$$
\begin{cases}-\hat{L} \psi \geq \gamma b(x) \psi & \text { on } \Omega_{1} \\ \psi=0 & \text { on } \partial \Omega_{1} .\end{cases}
$$


If we choose

$$
0<\rho<\gamma^{\frac{1}{\sigma-1}}\left[\sup _{\Omega_{1}} \psi\right]^{-1}
$$

then the $C^{2, \mu}$ function $v_{-}=\rho \psi$ solves

$$
\left\{\begin{array}{l}
\Delta v_{-}+\hat{q}(x) v_{-}-b(x) v_{-}^{\sigma} \geq 0 \quad \text { on } \Omega_{1}, \\
v_{-}>0 \quad \text { on } \Omega_{1}, \quad v_{-}=0 \quad \text { on } \partial \Omega_{1} .
\end{array}\right.
$$

On the other hand, since $\lambda_{1}^{\hat{L}}\left(B_{0}\right)>0$, by Proposition 2.33 there exists $0<v_{+} \epsilon$ $C^{2, \mu}\left(\bar{\Omega}_{1}\right)$ satisfying

$$
\left\{\begin{array}{l}
\Delta v_{+}+\hat{q}(x) v_{+}-b(x) v_{+}^{\sigma} \leq 0 \quad \text { on } \Omega_{1}, \\
v_{+} \geq 0 \quad \text { on } \partial \Omega_{1} .
\end{array}\right.
$$

By the comparison Proposition 2.32, $v_{-} \leq v_{+}$on $\Omega_{1}$. Thus, by the monotone iteration scheme (see [Sat72], or [Kur89] for a different approach), we find a solution $w \in C^{2, \mu}\left(\bar{\Omega}_{1}\right)$ of the problem

$$
\left\{\begin{array}{l}
\Delta w+\hat{q}(x) w-b(x) w^{\sigma}=0 \quad \text { on } \Omega_{1}, \\
w>0 \quad \text { on } \Omega_{1}, \quad w=0 \quad \text { on } \partial \Omega_{1} .
\end{array}\right.
$$

Note that $w>0$ on $\partial \Omega$ since $\bar{\Omega} \subset \Omega_{1}$. We set $w_{+}=\xi w, \xi>0$. Then, it is immediate to see that, since $\hat{q} \geq q$ on $\bar{\Omega}$, up to choosing $\xi$ sufficiently large

$$
\left\{\begin{array}{l}
\Delta w_{+}+q(x) w_{+}-b(x) w_{+}^{\sigma} \leq 0 \quad \text { on } \Omega, \\
w_{+} \geq n \quad \text { on } \partial \Omega .
\end{array}\right.
$$

Since $u \equiv 0$ is clearly a subsolution of the same problem, by the monotone iteration scheme we deduce the existence of a non-negative solution $u \in C^{2, \mu}(\bar{\Omega})$ of the problem (2.68). However, $u>0$. Indeed, $\Delta u+\left(q(x)-b(x) u^{\sigma-1}\right) u=0$ and now apply the strong maximum principle ([GT98], p.35) to conclude.

In the next result we produce a solution blowing up at the boundary of $\Omega$.

LEMma 2.35. In the assumptions of Lemma 2.34, there exists a solution $u \in$ $C_{\mathrm{loc}}^{2, \mu}(\Omega)$ of the problem

$$
\left\{\begin{array}{l}
\Delta u+q(x) u-b(x) u^{\sigma}=0 \quad \text { on } \Omega, \\
u>0 \quad \text { on } \Omega, \quad u \rightarrow+\infty \quad \text { on } \partial \Omega .
\end{array}\right.
$$

Proof. By standard Schauder estimates (Chapter 6 of [GT98]), it is enough to show that the sequence $\left\{u_{n}\right\}, n \in \mathbb{N}$, with $u_{n}$ solution of $(2.68)$, is bounded on any compact subset $K$ of $\Omega$. Once this is proved, by Theorem 6.2 of [GT98] $\left\{u_{n}\right\}$ is bounded in $C^{2, \mu}(K)$ for every domain $K$ with compact closure in $\Omega$. AscoliArzelà compactness result together with a Cantor diagonal argument yields, up to a subsequence, $u_{n} \rightarrow u$ in the $C^{2}$ topology. As a matter of fact, $u$ is again in $C_{\text {loc }}^{2, \mu}(\Omega)$ by passing to the limit in the definition of Hölder seminorm. If $K \subset \Omega \backslash B_{0}$, then we can find a finite covering of relatively compact balls $\left\{B_{i}\right\}$ for $K, i \in\{1, \ldots, t\}$, such that $b(x)>0$ on $\bar{B}_{i}$. We claim that for each $B_{i}$ there exists a constant $C_{i}$ such that $u_{n} \leq C_{i}$ on $B_{i}$ for every $n \in \mathbb{N}$. Postponing for a moment the proof of this claim we deduce the existence of a constant $C$ such that

$$
u_{n}(x) \leq C \quad \forall x \in K, \forall n \in \mathbb{N} .
$$


It remains to find an upper bound for $u_{n}$ in a neighbourhood of $B_{0}$. Towards this aim, let $\left\{N_{j}\right\}$ be a decreasing nested sequence of relatively compact domains with smooth boundary converging to $B_{0}$. By the compactness of $B_{0}$, we can choose $j$ sufficiently large that $\bar{N}_{j} \subset \Omega$. Furthermore, by the definition of $\lambda_{1}^{L}\left(B_{0}\right)$ and by $\lambda_{1}^{L}\left(B_{0}\right)>0$, we can choose $j$ big enough in such a way that

$$
\lambda_{1}^{L}\left(N_{j}\right)>0
$$

is met. Now, $\partial N_{2 j}$ is compact, therefore (2.72) holds on $\partial N_{2 j}$ for some constant $C_{2}>0$. Let $\varphi$ be the positive eigenfunction associated to $\lambda_{1}^{L}\left(N_{j}\right)$. Then, there exists a positive constant $S>0$ such that

$$
S \varphi \geq C_{2} \geq u_{n} \quad \text { on } \partial N_{2 j}, \quad \forall n \in \mathbb{N} .
$$

Since, on $N_{2 j}$,

$$
\begin{aligned}
& \Delta(S \varphi)+q(x)(S \varphi)=-\lambda_{1}^{L}\left(N_{j}\right)(S \varphi)<0 ; \\
& \Delta u_{n}+q(x) u_{n}=b(x) u_{n}^{\sigma} \geq 0,
\end{aligned}
$$

we can apply Proposition 2.32 with $b(x) \equiv 0$ to deduce the uniform estimate $u_{n} \leq$ $S \varphi \leq S\|\varphi\|_{L^{\infty}\left(N_{j}\right)}$ on $N_{2 j}$.

To finish the proof of of the Lemma is remains to prove the claim. Let $B_{3 R}$ be a relatively compact ball of radius $3 R$ such that $b(x)>0$ on $\bar{B}_{3 R}$. Let $u>0$ be a solution of

$$
\Delta u+q(x) u=b(x) u^{\sigma} \quad \text { on } B_{3 R} .
$$

Then, if $q_{0}=\|q(x)\|_{L^{\infty}\left(B_{3 R}\right)}, u$ satisfies

$$
\Delta u+q_{0} u \geq 0 .
$$

Thus, we can apply Theorem 8.17 of $[\mathbf{G T 9 8}]$ to the operator $\Delta+q_{0}$ to deduce the weak Harnack inequality

$$
\sup _{B_{R}} u \leq C\|u\|_{L^{p}\left(B_{2 R}\right)},
$$

for some $p>1$ and with a constant $C$ depending on $m, p, q_{0}, R$, the geometry of $B_{2 R}$ and the ellipticity constant of $\Delta$ on $B_{3 R}$. To give a uniform upper estimate of $\|u\|_{L^{p}\left(B_{2 R}\right)}$, observe that if $\phi \in C_{c}^{\infty}\left(B_{3 R}\right), \phi \equiv 1$ on $B_{2 R}$ and we choose $p=\sigma+1$, for any $\eta>1$

$$
\|u\|_{L^{p}\left(B_{2 R}\right)}^{p} \leq \int_{B_{3 R}} u^{\sigma+1} \phi^{\eta}
$$

It is therefore enough to give a uniform upper bound for the RHS of the above. Set

$$
\eta=\frac{2(\sigma+1)}{\sigma-1}>2
$$

and note that $\eta$ is twice the Hölder conjugate of $(\sigma+1) / 2$. Multiply both sides of

$$
\Delta u+q(x) u=b(x) u^{\sigma}
$$

by $u \phi^{\eta}$ and integrating by parts we get

$$
\int b(x) u^{\sigma+1} \phi^{\eta}=\int q u^{2} \phi^{\eta}-\int \phi^{\eta}|\nabla u|^{2}-\int \eta \phi^{\eta-1} u\langle\nabla u, \nabla \phi\rangle
$$

Set $b_{0}=\inf _{B_{3 R}} b>0$. An application of Cauchy-Schwarz and Young inequalities to the RHS gives

$$
(\mathrm{RHS}) \leq q_{0} \int u^{2} \phi^{\eta}+\frac{\eta^{2}}{4} \int \phi^{\eta-2} u^{2}|\nabla \phi|^{2} .
$$


We now apply Hölder's inequality to both terms of the RHS to get

$$
\begin{aligned}
b_{0} \int u^{\sigma+1} \phi^{\eta} \leq \int b(x) u^{\sigma+1} \phi^{\eta} \leq & q_{0}\left\{\int u^{\sigma+1} \phi^{\eta}\right\}^{\frac{2}{\sigma+1}}\left\{\int \phi^{\eta}\right\}^{\frac{\sigma-1}{\sigma+1}} \\
& +\frac{\eta^{2}}{4}\left\{\int u^{\sigma+1} \phi^{\eta}\right\}^{\frac{2}{\sigma+1}}\left\{\int|\nabla \phi|^{\eta}\right\}^{\frac{\sigma-1}{\sigma+1}} .
\end{aligned}
$$

Simplifying, we obtain

$$
\int u^{\sigma+1} \phi^{\eta} \leq \frac{1}{b_{0}}\left[q_{0}\left\{\int \phi^{\eta}\right\}^{\frac{\sigma-1}{\sigma+1}}+\frac{\eta^{2}}{4}\left\{\int|\nabla \phi|^{\eta}\right\}^{\frac{\sigma-1}{\sigma+1}}\right]^{\frac{\sigma+1}{\sigma-1}},
$$

and the uniform $L^{\sigma+1}$-estimate follows.

REMARK 2.36. For a proof of $L^{\infty}$ estimates with a different method inspired by a work of L. Ahlfors [Ahl38], the reader can consult the Appendix of [RRV97].

Lemma 2.37. Let $q(x), b(x) \in C_{\text {loc }}^{0, \mu}(M), \mu \in(0,1], b(x) \geq 0$ and suppose that $B_{0}$ is compact and satisfies $\lambda_{1}^{L}\left(B_{0}\right)>0$. If $u_{-} \in C_{\mathrm{loc}}^{2, \mu}(M), u \geq 0, u \neq 0$ is a global subsolution of

$$
\Delta u+q(x) u-b(x) u^{\sigma}=0, \quad \sigma>1
$$

on $M$, then (2.74) has a maximal positive $C_{\text {loc }}^{2, \mu}$ solution on $M$.

Proof. We fix an exhausting sequence $\left\{\Omega_{k}\right\}$ of relatively compact open domains with smooth boundary such that

$$
B_{0} \Subset \Omega_{k} \Subset \Omega_{k+1} \quad \forall k \in \mathbb{N} .
$$

Having fixed $k$, according to Lemma 2.35 we can construct a blowing up solution $0<u_{k} \in C_{\text {loc }}^{2, \mu}\left(\Omega_{k}\right)$ of the problem (2.71) with $\Omega=\Omega_{k}$. Note that, by Proposition 2.32

$$
u_{k} \geq u_{-} \quad \text { on } \Omega_{k} \text {. }
$$

Similarly, $u_{k+1} \leq u_{k}$ on $\Omega_{k}$. Since $u_{k}$ is monotone decreasing, by elliptic regularity it converges locally in the $C^{2}$ topology to a $C_{\mathrm{loc}}^{2, \mu}$ solution of (2.74). Because of (2.75), $u \geq u_{-}$on $M$, ad since $u_{-} \neq 0$, by the maximum principle $u>0$ on $M$. If $\widetilde{u}>0$ is any non-negative $C^{2}$ solution of (2.74), by Proposition $2.32 \widetilde{u} \leq u_{k}$ on $\Omega_{k}$, so that letting $k \rightarrow+\infty$ we deduce $\widetilde{u} \leq u$. This proves that $u$ is maximal.

Proof of Theorem 2.30. By the above lemmas, assumption $\lambda_{1}^{L}\left(B_{0}\right)>0$ enables us to produce a positive maximal $C_{\text {loc }}^{2, \mu}$ solution $u$ provides we can find some non-negative, non zero subsolution $u_{-}$. The requirement $\lambda_{1}^{L}(M)<0$ is what we need to construct $u_{-}$. Indeed, we are going to produce as $u_{-}$the minimal positive solution $v$. The method follows the lines of that of Lemma 2.34, where the first step was a perturbation of $L$ to produce some operator $\hat{L}$ satisfying $\lambda_{1}^{\hat{L}}(M)<0$. Here, since $\lambda_{1}^{L}(M)<0$ we can fix a sufficiently large relatively compact set $\Omega$ with smooth boundary such that $\lambda_{1}^{L}(\Omega)<0$. Let $\varphi \in C^{2, \mu}(\bar{\Omega})$ be the corresponding normalized eigenfunction. If $\gamma$ is sufficiently small, then

$$
\int_{\Omega}|\nabla \varphi|^{2}-q(x) \varphi^{2}+\gamma b(x) \varphi^{2}=\lambda_{1}^{L}(\Omega)+\gamma \int_{\Omega} b(x) \varphi^{2}<0,
$$


thus $\lambda_{1}^{\widetilde{L}}(\Omega)<0$, where $\widetilde{L}=L+\gamma b(x)$. Let $\psi \in C^{2, \mu}(\bar{\Omega})$ be a positive eigenfunction corresponding to $\lambda_{1}^{\widetilde{L}}(\Omega)$. Then $\psi$ solves

$$
\left\{\begin{array}{l}
-L \psi \geq \gamma b(x) \psi \\
\psi=0 \quad \text { on } \partial \Omega .
\end{array}\right.
$$

If we choose $\rho \leq \gamma^{1 /(\sigma-1)}\left[\sup _{\Omega} \psi\right]^{-1}$, the function $v_{-}=\rho \psi$ solves

$$
\left\{\begin{array}{l}
\Delta v_{-}+q(x) v_{-} \geq b(x) v_{-}^{\sigma} \\
v_{-}=0 \quad \text { on } \partial \Omega .
\end{array}\right.
$$

Lemma 2.33 guarantees the existence of a positive $C^{2, \mu}$ supersolution $v_{+}$of $(2.76)$, which by Proposition 2.32 satisfies $v_{-} \leq v_{+}$. Then, the monotone iteration scheme and the maximum principle give a positive, $C^{2, \mu}$ solution $v$ with zero boundary condition on $\partial \Omega$. Choose now a sequence $\left\{\Omega_{k}\right\}$ of relatively compact domains with smooth boundaries, and let $v_{k}$ be the positive solution of $\Delta v_{k}+q(x) v_{k}-b(x) v_{k}^{\sigma}=$ 0 with zero condition on $\partial \Omega_{k}$ constructed above. By Proposition 2.32, $\left\{v_{k}\right\}$ is monotone increasing, and uniformly bounded by the procedure of Lemma 2.35. Thus the elliptic estimates, together with Ascoli-Arzelà and Cantor arguments yield $C^{2}$ convergence of $\left\{v_{k}\right\}$ to a $C^{2, \mu}$ solution $v>0$, which is obviously minimal, since by Proposition 2.32 every positive solution $w$ shall satisfy $w \geq v_{k}$ on $\Omega_{k}$. This concludes the proof.

REMARK 2.38. If $\lambda_{1}^{L}(M) \geq 0$, it is possible to prove the triviality of any solution $u \in \operatorname{Lip}_{\text {loc }}(M), u \geq 0$ of

$$
u \Delta u+q(x) u^{2}-b(x) u^{\sigma+1} \geq 0
$$

satisfying suitable integrability assumptions. As a consequence, the spectral assumption $\lambda_{1}^{L}(M)<0$ in Theorem 2.30 is necessary. We will come back to this nonexistence result in Section 4.3, when we will prove a sharp Liouville type theorem on manifolds with a pole as a consequence of our ODE approach. Liouville type theorems are a cornerstone in modern Differential Geometry and Geometric Analysis. For a detailed treatment, together with many geometric applications, see [PRS08] and the references therein. 


\section{CHAPTER 3}

\section{On the solutions of the ODE $\left(v z^{\prime}\right)^{\prime}+A v z=0$}

The purpose of this chapter is to introduce one of the main tools in our investigation of the $\operatorname{ODE}\left(v z^{\prime}\right)^{\prime}+A v z=0$ : the critical curve $\chi(r)$. After a few brief introductory considerations, we proceed discussing some of its properties related to geometry. In particular, we focus on comparison results for $\chi$ and we discuss the behaviour of $\chi$ as $r \rightarrow+\infty$ depending on some relevant geometric quantities.

\subsection{Existence, uniqueness and the behaviour of zeroes}

This preliminary section is devoted to showing existence, in the Lip loc $_{\text {class, of }}$ a solution of the Cauchy problem

$$
\left\{\begin{array}{l}
\left(v(r) z^{\prime}(r)\right)^{\prime}+A(r) v(r) z(r)=0 \quad \text { on } \mathbb{R}^{+} \\
z^{\prime}(r)=O(1) \quad \text { as } r \downarrow 0^{+}, \quad z(0)=z_{0}>0
\end{array}\right.
$$

under the assumptions

(A1) $\quad A(r) \in L_{\mathrm{loc}}^{\infty}\left(\mathbb{R}_{0}^{+}\right)$

$$
\begin{aligned}
& 0 \leq v(r) \in L_{\mathrm{loc}}^{\infty}\left(\mathbb{R}_{0}^{+}\right), \quad \frac{1}{v(r)} \in L_{\mathrm{loc}}^{\infty}\left(\mathbb{R}^{+}\right), \quad \lim _{r \rightarrow 0^{+}} v(r)=0 \\
& v(r) \int_{r}^{a} \frac{\mathrm{d} s}{v(s)} \text { and } \frac{1}{v(r)} \int_{0}^{r} v(s) \mathrm{d} s \in L^{\infty}([0, a]), \quad \text { for some } a \in \mathbb{R}^{+} \\
& \frac{1}{v(r)} \int_{0}^{r} v(x) \mathrm{d} x=o(1) \quad \text { as } r \rightarrow 0^{+} .
\end{aligned}
$$

Clearly, (V3) and the third assumption in (V1) require the choice of a version of $v$.

REMARK 3.1. Both (V2) and (V3) are met if, for instance, a version of $v$ is nondecreasing on $(0, a)$. By Proposition 1.6, this is always the case if $v(r)=\operatorname{vol}\left(\partial B_{r}\right)$ and $a$ is sufficiently small.

Solving (3.1) is equivalent to finding $z \in \operatorname{Lip}_{\text {loc }}\left(\mathbb{R}_{0}^{+}\right)$satisfying

$$
z(r)=z_{0}-\int_{0}^{r} \frac{1}{v(s)}\left\{\int_{0}^{s} A(x) v(x) z(x) \mathrm{d} x\right\} \mathrm{d} s .
$$

Observe that $z^{\prime}(r)=O(1)$ near 0 is automatically true if $z \in \operatorname{Lip}_{\text {loc }}\left(\mathbb{R}_{0}^{+}\right)$. Next, with a suitable substitution we prove both uniqueness and the fact that the zeros $z(r)$ if any, are at isolated points. Existence results for the Sturm-Liouville problem (3.1) are classical and proved with fairly weaker regularity on $A$ and $v$, for instance, in Section 2 of the Lecture Notes of J. Weidmann [Wei87]. However, to keep the paper self-contained, we report here a direct proof for the Lip loc class. As usual, this relies upon the Banach-Caccioppoli fixed point theorem, together with an AscoliArzelá argument to deal with the singularity in $r=0$. 
Proposition 3.2 (Existence). Under assumptions (A1), (V1), (V2) there exists a solution $z(r) \in \operatorname{Lip}_{\text {loc }}\left(\mathbb{R}_{0}^{+}\right)$of

$$
\left\{\begin{array}{l}
\left(v(r) z^{\prime}(r)\right)^{\prime}+A(r) v(r) z(r)=0 \quad \text { on } \mathbb{R}^{+} \\
z(0)=z_{0}>0 .
\end{array}\right.
$$

Moreover, if also (V3) holds, up to a zero-measure set $\Omega, z^{\prime}(r) \rightarrow 0$ as $r \rightarrow 0, r \notin \Omega$. If $v$ is continuous, $z \in C^{1}\left(\mathbb{R}_{0}^{+}\right)$and, when (V3) is met, $z^{\prime}(0)=0$; if $A \in C^{k}\left(\mathbb{R}_{0}^{+}\right)$, $k \geq 0, v \in C^{k+1}\left(\mathbb{R}_{0}^{+}\right)$, then $z \in C^{k+2}\left(\mathbb{R}^{+}\right)$.

Proof. Assume $A \not \equiv 0$ in $L_{\text {loc }}^{\infty}$ sense, the case $A \equiv 0$ being easier. First, fix a sequence $R_{j} \uparrow+\infty$. We can suppose that $a \in\left(0, R_{j}\right)$ for every $j$, where $a$ is as in (V2), and $A \not \equiv 0$ on $\left[0, R_{j}\right]$. Fix $\varepsilon \in(0, a)$, and define

$$
v_{\varepsilon}(r)= \begin{cases}v(\varepsilon) & \text { on }(0, \varepsilon] \\ v(r) & \text { on }[\varepsilon,+\infty)\end{cases}
$$

Then,

$$
k_{\varepsilon}(r, s)=-A(s) v_{\varepsilon}(s) \int_{s}^{r} \frac{\mathrm{d} x}{v_{\varepsilon}(x)}
$$

belongs to $L_{\text {loc }}^{\infty}\left(\mathbb{R}_{0}^{+} \times \mathbb{R}_{0}^{+}\right)$. Thus, by the Banach-Caccioppoli theorem (for instance, one can consult chapter IX of [KF80]), Volterra integral equation of the second kind

$$
w(r)=z_{0}+\int_{0}^{r} k_{\varepsilon}(r, s) w(s) \mathrm{d} s
$$

restricted to every interval $\left[0, R_{j}\right]$ where the kernel $k_{\varepsilon}(r, s)$ is bounded, admits a unique solution $z_{\varepsilon, j} \in L^{2}\left(\left(0, R_{j}\right)\right)$. From (3.4), an integration by parts applied to the integrable function $-A(s) v_{\varepsilon}(s) z_{\varepsilon, j}(s)$ and to the absolutely continuous one

$$
\int_{s}^{r} \frac{\mathrm{d} x}{v_{\varepsilon}(x)}
$$

gives

$$
z_{\varepsilon, j}(r)=z_{0}-\int_{0}^{r} \frac{1}{v_{\varepsilon}(s)}\left\{\int_{0}^{s} A(x) v_{\varepsilon}(x) z_{\varepsilon, j}(x) \mathrm{d} x\right\} \mathrm{d} s
$$

on $\left[0, R_{j}\right]$. This shows that $z_{\varepsilon, j}(r)$, being an integral function, is absolutely continuous on $\left[0, R_{j}\right]$, hence differentiable a.e. with derivative

$$
-\frac{1}{v_{\varepsilon}(r)} \int_{0}^{r} A(x) v_{\varepsilon}(x) z_{\varepsilon, j}(x) \mathrm{d} x \in L^{\infty}\left(\left[0, R_{j}\right]\right) .
$$

Therefore, $z_{\varepsilon, j}(r)$ is a Lipschitz function on $\left[0, R_{j}\right]$. By the uniqueness of solutions of (3.5), we deduce that the functions $\left\{z_{\varepsilon, j}\right\}_{j}$ fit together on common intervals to give a locally Lipschitz solution $z_{\varepsilon}(r)$ on $\mathbb{R}_{0}^{+}$. What we want to prove is that, for every $R_{j}$, the family $\left\{z_{\varepsilon}\right\}_{\varepsilon \in(0, a)}$ is equibounded and equi-Lipschitz in $C^{0}\left(\left[0, R_{j}\right]\right)$. For the ease of notation, from now on we omit the subscript $j$ and we consider the problem on $[0, R] \subset \mathbb{R}_{0}^{+}$. For every $s \leq \varepsilon$ observe that, because of (A1), (V2) and the definition of $v_{\varepsilon}$,

$$
v_{\varepsilon}(s) \int_{s}^{a} \frac{\mathrm{d} x}{v_{\varepsilon}(x)}=v(\varepsilon)\left[\int_{s}^{\varepsilon} \ldots+\int_{\varepsilon}^{a} \ldots\right] \leq(\varepsilon-s)+v(\varepsilon) \int_{\varepsilon}^{a} \frac{\mathrm{d} x}{v(x)},
$$


hence

$$
\left\|v_{\varepsilon}(\cdot) \int^{a} \frac{\mathrm{d} x}{v_{\varepsilon}(x)}\right\|_{L^{\infty}([0, a])} \leq 2 a+\left\|v(\cdot) \int^{a} \frac{\mathrm{d} x}{v(x)}\right\|_{L^{\infty}([0, a])} \leq C
$$

For some uniform constant $C$ independent of $\varepsilon$. Thus, for $0 \leq s \leq r \leq a$ we have

$$
\left|k_{\varepsilon}(r, s)\right| \leq C\|A\|_{L^{\infty}([0, R])} .
$$

Next, we consider the case $0 \leq s \leq a<r \leq R$. Because of (V1), on $[a, R] v^{-1}$ is bounded. It follows that

$$
\begin{aligned}
\left|k_{\varepsilon}(r, s)\right| & =A(s) v_{\varepsilon}(s)\left\{\int_{s}^{a} \frac{\mathrm{d} x}{v_{\varepsilon}(x)}+\int_{a}^{r} \frac{\mathrm{d} x}{v(x)}\right\} \\
& \leq\|A\|_{L^{\infty}([0, R])}\left(C+\|v\|_{L^{\infty}([0, R])}\left\|v^{-1}\right\|_{L^{\infty}([a, R])} R\right)
\end{aligned}
$$

The case $0<a \leq s \leq r \leq R$ is immediate:

$$
\left|k_{\varepsilon}(r, s)\right| \leq\|A\|_{L^{\infty}([0, R])}\|v\|_{L^{\infty}([0, R])}\left\|v^{-1}\right\|_{L^{\infty}([a, R])} R .
$$

Therefore, there exists $L=L(R, a)>0$ such that

$$
\sup _{\varepsilon \in(0, a)}\left(\sup _{0 \leq s \leq r \leq R}\left|k_{\varepsilon}(r, s)\right|\right) \leq L
$$

Using (3.9) into (3.5) and applying Gronwall's lemma we conclude

$$
\left|z_{\varepsilon}(r)\right| \leq z_{0} e^{L r} \leq z_{0} e^{L R} \quad \text { on }[0, R]
$$

This shows equiboundedness of the family $\left\{z_{\varepsilon}\right\}_{\varepsilon \in(0, a)}$. To show equicontinuity we differentiate (3.6) to obtain

$$
z_{\varepsilon}^{\prime}(r)=-\frac{1}{v_{\varepsilon}(r)} \int_{0}^{r} A(x) v_{\varepsilon}(x) z_{\varepsilon}(x) \mathrm{d} x \quad \text { a.e. on }[0, R] .
$$

As in (3.7), using (V2) it is easy to see that there exists a constant $C>0$, independent of $\varepsilon$, such that

$$
\left\|\frac{1}{v_{\varepsilon}(\cdot)} \int_{0}^{\cdot} v_{\varepsilon}(x) \mathrm{d} x\right\|_{L^{\infty}([0, R])} \leq C
$$

whence

$$
\left|z_{\varepsilon}^{\prime}(r)\right|=\|A\|_{L^{\infty}([0, R])} C\left\|z_{\varepsilon}\right\|_{L^{\infty}([0, R])} \leq C r_{0} e^{L R} \quad \text { a.e. on } \quad[0, R]
$$

This shows that $\left\{z_{\varepsilon}\right\}_{\varepsilon \in(0, a)}$ is equi-Lipschitz on every compact subset $[0, R] \subset \mathbb{R}_{0}^{+}$. The Ascoli-Arzelá theorem and a Cantor diagonal argument on increasing intervals yields a sequence $\left\{z_{\varepsilon_{n}}\right\}_{n}$ which converges locally uniformly to a locally Lipschitz function $z$ on $\mathbb{R}_{0}^{+}$. Clearly, $v_{\varepsilon_{n}} \rightarrow v$ in $L^{\infty}\left(\mathbb{R}_{0}^{+}\right)$. If we set

$$
w_{\varepsilon}(s)=\frac{1}{v_{\varepsilon}(s)} \int_{0}^{s} A(x) v_{\varepsilon}(x) z_{\varepsilon}(x) \mathrm{d} x
$$

using (3.11) and (3.12) we see that $w_{\varepsilon_{n}}$ is locally a bounded sequence of $L_{\text {loc }^{-}}^{\infty}$ functions converging pointwise to

$$
w(s)=\frac{1}{v(s)} \int_{0}^{s} A(x) v(x) z(x) \mathrm{d} x \quad \text { a.e. on } \mathbb{R}_{0}^{+}
$$


By the dominated convergence theorem, for every $r \in R^{+} w_{\varepsilon_{n}} \rightarrow w$ in $L^{1}((0, r])$, hence

$$
\lim _{n \rightarrow+\infty} \int_{0}^{r} \frac{\mathrm{d} s}{v_{\varepsilon_{n}}(s)}\left\{\int_{0}^{s} A(x) v_{\varepsilon_{n}}(x) z_{\varepsilon_{n}}(x) \mathrm{d} x\right\}=\int_{0}^{r} \frac{\mathrm{d} s}{v(s)}\left\{\int_{0}^{s} A(x) v(x) z(x) \mathrm{d} x\right\}
$$

Because of (3.6) it follows that $z$ satisfies the integral equation

$$
z(r)=z_{0}-\int_{0}^{r} \frac{1}{v(s)}\left\{\int_{0}^{s} A(x) v(x) z(x) \mathrm{d} x\right\} \mathrm{d} s
$$

hence the Cauchy problem (3.3). Note that, when $v(r), A(r)$ are also continuous, from (3.13) we deduce that $z(r) \in C^{1}\left(\mathbb{R}^{+}\right)$. This concludes the first part of the proof. Under the additional assumption (V3),

$$
\left|z^{\prime}(r)\right| \leq\|A\|_{L^{\infty}([0, a])}\|z\|_{L^{\infty}([0, a])}\left|\frac{1}{v(r)} \int_{0}^{r} v(s) \mathrm{d} s\right| \rightarrow 0^{+} \quad \text { as } r \rightarrow 0^{+},
$$

and this concludes the second part, while $C^{k+2}$ regularity follows easily from (3.13) by iteration.

REMARK 3.3. With a minor modification of the above argument we can provide existence of a locally Lipschitz solution of the problem

$$
\left\{\begin{array}{l}
\left(v(r) z^{\prime}(r)\right)^{\prime}+A(r) v(r) z(r)=0 \quad \text { on }\left[r_{0},+\infty\right) \\
z\left(r_{0}\right)=z_{0} \in \mathbb{R} .
\end{array}\right.
$$

when $(\mathrm{A} 1)$ and $(\mathrm{V} 1)$ are met on $\left[r_{0},+\infty\right)$, for some $r_{0}>0$. Note that $1 / v$ is required to be bounded also in a neighborhood of $r_{0}$.

REMARK 3.4. We observe that Sturm type arguments can be easily rephrased for $\left(v z^{\prime}\right)^{\prime}+A v z=0$. Indeed, if $z_{1}, z_{2}$ denotes solutions of (3.3) with, respectively, potential $A_{1}$ and $A_{2}$, it is enough to differentiate $F=\left(v z_{1}^{\prime}\right) z_{2}-\left(v z_{2}^{\prime}\right) z_{1}$ and to proceed analogously to the proof of Theorem 1.9. Therefore, the properties of being oscillatory and nonoscillatory are well defined and mutually exclusive also for $\operatorname{Lip}_{\text {loc }}$ solutions of $\left(v z^{\prime}\right)^{\prime}+A v z=0$.

COROllary 3.5 (Existence and uniqueness). Under assumptions (A1), (V1), $(\mathrm{V} 2)$, there exists a unique solution $z(r) \in \operatorname{Lip}_{\text {loc }}\left(\mathbb{R}_{0}^{+}\right)$of the problem

$$
\left\{\begin{array}{l}
\left(v(r) z^{\prime}(r)\right)^{\prime}+A(r) v(r) z(r)=0 \quad \text { on } \mathbb{R}^{+} \\
z\left(0^{+}\right)=z_{0}>0
\end{array}\right.
$$

Proof. If $z_{1}, z_{2}$ are two distinct solutions of the Cauchy problem, by Sturm argument they coincide on some interval $[0, \delta)$. Fix $R_{j} \uparrow+\infty$. Since the Cauchy problem on $I_{j}=\left[\delta / 2, R_{j}\right)$ with initial data $\left(v z^{\prime}\right)(\delta / 2)=\left(v z_{1}^{\prime}\right)(\delta / 2)$ is equivalent to a Volterra integral equation with locally bounded kernel, by uniqueness $\left(z_{1}\right)_{\mid I_{j}} \equiv$ $\left(z_{2}\right)_{\mid I_{j}}$ is the unique solution on each $I_{j}$, hence on $\mathbb{R}^{+}$.

The next proposition ensures that zeros of $z(r)$, if any, cannot have cluster points on $\mathbb{R}^{+}$. Note that usual methods cannot be directly applied to $z$ since $z$ is not $C^{1}$, and we first need a suitable substitution.

Proposition 3.6 (Isolated zeroes). Assume (A1) and (V1). Then, the zeros of every solution $z(r) \in \operatorname{Lip}_{\text {loc }}\left(\mathbb{R}_{0}^{+}\right)$of $(3.3)$, if any, are at isolated points of $\mathbb{R}_{0}^{+}$. 
Proof. If $1 / v \in L^{1}(+\infty)$, we set

$$
s(r)=\left(\int_{r}^{+\infty} \frac{\mathrm{d} s}{v(s)}\right)^{-1} .
$$

Clearly $s: \mathbb{R}^{+} \rightarrow I=\left(s_{0},+\infty\right)$ is a locally bi-Lipschitz bijection, where $s_{0} \geq 0$. We let $r(s)$ be the inverse function and we define $g(s)=s z(r(s))$, as classically done, for instance, in [Lei50], [Moo55]. A first differentiation shows that $g \in C_{\mathrm{loc}}^{1,1}(I)$, and a further differentiation together with (3.3) shows that $g$ solves

$$
\frac{\mathrm{d}^{2} g}{\mathrm{~d} s^{2}}+\left(\frac{A(r(s)) v^{2}(r(s))}{s^{4}}\right) g=0 .
$$

If the zeroes of $g$ have a cluster point $\widetilde{s}$ on $I$, by Rolle theorem $g(\widetilde{s})=g^{\prime}(\widetilde{s})=0$. By the uniqueness of solutions of the Volterra integral equation associated to

$$
\left\{\begin{array}{l}
\frac{\mathrm{d}^{2} g}{\mathrm{~d} s^{2}}+\left(\frac{A(r(s)) v^{2}(r(s))}{s^{4}}\right) g=0, \\
g(\widetilde{s})=g^{\prime}(\widetilde{s})=0,
\end{array}\right.
$$

we deduce that $g \equiv 0$ on $\left(s_{0},+\infty\right)$, and therefore that $z \equiv 0$, which contradicts $z(0)=z_{0}>0$. Thus, the zeroes of $g$ are isolated on $I$, and by (3.15) it follows that also those of $z(r)$ are isolated on $\mathbb{R}^{+}$. When $1 / v \notin L^{1}(+\infty)$, since $z_{0}>0$ we can fix $R>0$ sufficiently small that $z>0$ on $[0, R]$. The above argument applies after the change of variables

$$
s(r)=\int_{R}^{r} \frac{\mathrm{d} s}{v(s)} \quad \text { and } \quad g(s)=z(r(s)) .
$$

Indeed, $s$ is a bi-Lipschitz bijection from $[R,+\infty)$ to $\mathbb{R}_{0}^{+}$, and $g \in C_{\text {loc }}^{1,1}\left(\mathbb{R}_{0}^{+}\right)$solves

$$
\frac{\mathrm{d}^{2} g}{\mathrm{~d} s^{2}}+A(r(s)) v^{2}(r(s)) g=0 .
$$

From Proposition 1.6, (V1), (V2), (V3) are met when $v(r)$ is the volume growth of geodesic spheres of a complete, non-compact Riemannian manifold.

Corollary 3.7. Let $(M,\langle\rangle$,$) be a complete, non-compact Riemannian man-$ ifold of dimension $m$, and let $K \subset M$ be either a point or a compactly embedded submanifold satisfying $\operatorname{dim}(K) \leq m-2$. Define $B_{r}$ to be the geodesic ball centered at $K$, and let $v(r)=\operatorname{vol}\left(\partial B_{r}\right)$. Then, for every $A(r)$ satisfying (A1), there exists a unique solution $z \in \operatorname{Lip}_{\text {loc }}\left(\mathbb{R}_{0}^{+}\right)$of problem (3.3). Moreover, $z$ is $C^{1}$ in a neighbourhood of $0, z^{\prime}(0)=0$ and $z$ has isolated zeroes. Analogously, for each $z_{0}^{\prime} \in \mathbb{R}$ there exists a unique solution of problem (3.14) satisfying also $z^{\prime}\left(r_{0}\right)=z_{0}^{\prime}$ and, if $z \not \equiv 0$, $z$ has isolated zeroes.

REMARK 3.8. When $K$ is a compact hypersurface, the compactness argument in Proposition 1.6 is not necessary since $v(0)>0$, and existence is easier to prove. In this case, uniqueness follows once we also specify $z^{\prime}(0)$.

REMARK 3.9. Of course, the set of, say, Lip ${ }_{\text {loc }}$ solutions of $\left(v z^{\prime}\right)^{\prime}+A v z=0$ on $[R,+\infty), R \geq 0$, is a linear space of dimension two. By general theory, if $z_{1}$ is 
a $\operatorname{Lip}_{\text {loc }}$ solution without zeroes on $[R,+\infty)$ then another $\operatorname{Lip}_{\text {loc }}$ solution, linearly independent of $z_{1}$, has the explicit expression

$$
z_{2}(r)=\left\{\begin{array}{cc}
z_{1}(r) \int_{R}^{r} \frac{\mathrm{d} s}{v(s) z_{1}^{2}(s)} & \text { if }\left(v z_{1}^{2}\right)^{-1} \in L^{1}\left(R^{+}\right) ; \\
z_{1}(r) \int_{R+1}^{r} \frac{\mathrm{d} s}{v(s) z_{1}^{2}(s)} & \text { if } \quad\left(v z_{1}^{2}\right)^{-1} \notin L^{1}\left(R^{+}\right) .
\end{array}\right.
$$

The classical change of variables exploited in the proof of Theorem 3.6 will be repeatedly used throughout the paper. For this reason, we state next proposition to avoid tiresome repetitions.

Proposition 3.10. Let $K \in L_{\mathrm{loc}}^{\infty}\left(\mathbb{R}_{0}^{+}\right)$, and let $g$ be a solution of

$$
\left\{\begin{array}{l}
g^{\prime \prime}+K(s) g=0 \quad \text { on } \mathbb{R}^{+} \\
g(0)=0, \quad g^{\prime}(0)=1 .
\end{array}\right.
$$

Choose $v$ satisfying (V1), (V2) and $1 / v \in L^{1}(+\infty) \backslash L^{1}\left(0^{+}\right)$, let $r=r(s)$ be the inverse function of

$$
s(r)=\left(\int_{r}^{+\infty} \frac{\mathrm{d} \tau}{v(\tau)}\right)^{-1}, \quad \text { and define } z(r)=\frac{g(s(r))}{s(r)} .
$$

Then, $z$ solves

$$
\left\{\begin{array}{l}
\left(v(r) z^{\prime}(r)\right)^{\prime}+\left(\frac{K(s(r)) s^{4}(r)}{v^{2}(r)}\right) v(r) z(r)=0 \quad \text { on } \mathbb{R}^{+}, \\
z(0)=1, \quad\left(v z^{\prime}\right)(0)=0 .
\end{array}\right.
$$

\subsection{The critical curve: definition and main estimates}

In what follows, when we deal with (3.3) or with the Cauchy problem

$$
\left\{\begin{array}{l}
\left(v(r) z^{\prime}(r)\right)^{\prime}+A(r) v(r) z(r)=0 \quad \text { on }\left[r_{0},+\infty\right) \\
z\left(r_{0}^{+}\right)=z_{0} \in \mathbb{R},
\end{array}\right.
$$

for some $r_{0}>0$, we always assume the validity of (A1) and (V1), with the understanding that, for (3.20), these requirements are met on $\left[r_{0},+\infty\right)$ and that $1 / v$ is bounded in a right neighbourhood of $r_{0}$. The critical curve $\chi$, in the form given below, has been introduced for the first time in [BMR09], and in some special cases in [BR97].

Throughout this section, we will require the further integrability condition

$$
\frac{1}{v(r)} \in L^{1}(+\infty) \text {. }
$$

This condition is essential for defining $\chi(r)$. As we shall see, the situation changes considerably when $1 / v$ is not integrable at infinity. We set

$$
\chi(r)=\left\{2 v(r) \int_{r}^{+\infty} \frac{\mathrm{d} s}{v(s)}\right\}^{-2}=\left\{\left(-\frac{1}{2} \log \int_{r}^{+\infty} \frac{\mathrm{d} s}{v(s)}\right)^{\prime}\right\}^{2} \in L_{\mathrm{loc}}^{\infty}\left(\mathbb{R}^{+}\right) .
$$


Observe that, for every fixed $r, \chi(r)$ depends on the behaviour of $v$ on the whole $[r,+\infty)$, but not on that before $r$. From the definition, it follows immediately that

$$
\int_{R}^{r} \sqrt{\chi(s)} \mathrm{d} s=\frac{1}{2} \log \left\{\left(\int_{R}^{+\infty} \frac{\mathrm{d} s}{v(s)}\right) /\left(\int_{r}^{+\infty} \frac{\mathrm{d} s}{v(s)}\right)\right\} \quad \forall 0<R<r
$$

whence, letting $r \rightarrow+\infty$, we deduce that, for every $v(r)$ satisfying (V1),

$$
\sqrt{\chi(r)} \notin L^{1}(+\infty)
$$

REMARK 3.11. We note in passing that, if $1 / v$ is integrable at zero, by (3.21) and (V1), $\chi\left(0^{+}\right)=+\infty$. The same happens when $v(r)$ satisfies (V1) and is increasing near zero, independently of its integrability at zero. Indeed, for every $a>0$ and $r \in(0, a)$,

$$
\frac{1}{2 \sqrt{\chi(r)}}=v(r) \int_{r}^{+\infty} \frac{\mathrm{d} s}{v(s)}=v(r)\left(\int_{r}^{a} \ldots+\int_{a}^{+\infty} \ldots\right) \leq(a-r)+C(a) v(r),
$$

for some constant $C(a)>0$, and the claim follows letting $r \rightarrow 0^{+}$by the arbitrariness of $a$.

Although the critical curve $\chi(r)$ is suitable to describe the oscillatory behavior of the ODE $\left(v z^{\prime}\right)^{\prime}+A v z=0$, it is in general not easy to handle, both because of its integral expression and for its lack of regularity. For geometric applications it is often useful to bound $v(r)=\operatorname{vol}\left(\partial B_{r}\right)$ from above or below by some function $f(r)$ with better regularity properties, and to introduce a critical curve $\chi_{f}$ associated to $f$ exactly as in (3.21) with $v(r)$ replaced by $f(r)$. Of course this is meaningful if $f$ satisfies requirements similar to those for $v(r)$. An important feature of $\chi_{f}$ is the homogeneity property $\chi_{C f} \equiv \chi_{f}$, for $C>0$. However, simple relations between $v$ and $f$ such as, for instance, $v \leq f$ do not imply similar relations between $\chi$ and $\chi_{f}$. Indeed, in this case a more stringent condition is required.

Proposition 3.12. Consider the functions $v, f$ on some open interval $I=$ $\left(r_{0},+\infty\right) \subset \mathbb{R}^{+}$. Then,

(i) If $v / f$ is non-increasing (resp. strictly decreasing) on $I, \chi(r) \leq \chi_{f}(r)$ (resp. $\left.\chi(r)<\chi_{f}(r)\right)$ on $I$

(ii) If $v / f$ is non-decreasing (resp. strictly increasing) on $I, \chi(r) \geq \chi_{f}(r)$ $\left(\right.$ resp. $\left.\chi(r)>\chi_{f}(r)\right)$ on $I$;

Proof. We consider case $(i)$, the second case being similar. Now $\chi \leq \chi_{f}$ on $I$ if and only if, for every $[R, r] \subset I$,

$$
\int_{R}^{r} \sqrt{\chi(s)} \mathrm{d} s \leq \int_{R}^{r} \sqrt{\chi_{f}(s)} \mathrm{d} s
$$

and because of (3.22) this is equivalent to

$$
h(r) \leq h(R) \quad \forall[R, r] \subset I
$$

where

$$
h(r)=\left\{\int_{r}^{+\infty} \frac{\mathrm{d} s}{f(s)}\right\} /\left\{\int_{r}^{+\infty} \frac{\mathrm{d} s}{v(s)}\right\} .
$$


By adapting the reasoning in [CGT82], p.42, if $v / f$ is non-increasing then $h(r)$ is non-increasing. Indeed,

$$
\begin{aligned}
\int_{r}^{\infty} \frac{1}{f} \int_{R}^{\infty} \frac{1}{v} & =\left[\int_{r}^{\infty}\left(\frac{1}{v}\right) \frac{v}{f}\right]\left[\int_{R}^{r} \frac{1}{v}\right]+\int_{r}^{\infty} \frac{1}{f} \int_{r}^{\infty} \frac{1}{v} \\
& \leq \frac{v(r)}{f(r)} \int_{r}^{\infty} \frac{1}{v} \int_{R}^{r} \frac{1}{v}+\int_{r}^{\infty} \frac{1}{f} \int_{r}^{\infty} \frac{1}{v} \\
& \leq\left[\int_{r}^{\infty} \frac{1}{v}\right]\left[\int_{R}^{r}\left(\frac{v}{f}\right) \frac{1}{v}\right]+\int_{r}^{\infty} \frac{1}{f} \int_{r}^{\infty} \frac{1}{v} \\
& =\int_{r}^{\infty} \frac{1}{v}\left[\int_{R}^{r} \frac{1}{f}+\int_{r}^{\infty} \frac{1}{f}\right]=\int_{r}^{\infty} \frac{1}{v} \int_{R}^{\infty} \frac{1}{f} .
\end{aligned}
$$

This proves (3.24). The above reasoning also shows that strict monotonicity of $v / f$ implies strict inequalities between $\chi$ and $\chi_{f}$, as can be easily checked.

As a consequence of the Bishop-Gromov comparison Theorem 1.24, the above result applies when $v(r)=\operatorname{vol}\left(\partial B_{r}\right)$ and $f$ is related to bounds on the Ricci tensor or on the radial sectional curvature.

Proposition 3.13 (Comparison for the critical curve). Let $(M,\langle\rangle$,$) be a com-$ plete manifold of dimension $m$ with a reference origin $o \in M$, and let $G \in C^{0}\left(\mathbb{R}_{0}^{+}\right)$. Define $\chi(r)$ as the critical curve associated to $v(r)=\operatorname{vol}\left(\partial B_{r}\right)$.

(i) Assume that

and let $g$ be a solution of

$$
\operatorname{Ricc}(\nabla r, \nabla r)(x) \geq-(m-1) G(r(x)) \quad \forall x \in M .
$$$$
\left\{\begin{array}{l}
g^{\prime \prime}-G g \geq 0 \quad \text { on } \mathbb{R}_{0}^{+}, \\
g(0)=0, \quad g^{\prime}(0)=1 .
\end{array}\right.
$$

Suppose that $g>0$ on $\mathbb{R}^{+}$. Then $\chi \leq \chi_{g^{m-1}}$ on $\mathbb{R}^{+}$.

(ii) Assume that

$$
\operatorname{cut}(o)=\emptyset, \quad K_{\mathrm{rad}}(x) \leq-G(r(x)) \quad \text { on } M .
$$

and let $g$ be a solution of

$$
\left\{\begin{array}{l}
g^{\prime \prime}-G g \leq 0 \quad \text { on } \mathbb{R}_{0}^{+}, \\
g(0)=0, \quad g^{\prime}(0)=1 .
\end{array}\right.
$$

Suppose that $g>0$ on $\mathbb{R}^{+}$. Then $\chi \geq \chi_{g^{m-1}}$ on $\mathbb{R}^{+}$.

We now describe explicit examples of critical curves $\chi$ and $\chi_{f}$ in some interesting cases. Unfortunately, as already observed the defining expression (3.21) is computationally difficult to handle. For this reason, explicit expressons can be found only in few simple situations. Thus, in the general case we shall limit ourselves to stress the asymptotic behaviour of $\chi$ near $0^{+}$and $+\infty$. We concentrate on the case $v(r)=\operatorname{vol}\left(\partial B_{r}\right)$. From the asymptotic $v(r) \sim \omega_{m-1} r^{m-1}$ as $r \rightarrow 0^{+}$in (1.63), a straightforward computation using De l'Hopital theorem yields

$$
\begin{array}{ll}
\chi(r) \sim \frac{(m-2)^{2}}{4 r^{2}} & \text { as } r \rightarrow 0^{+}, \text {if } m \geq 3 \\
\chi(r) \sim \frac{1}{4 r^{2} \log ^{2} r} \quad \text { as } r \rightarrow 0^{+}, \text {if } m=2 .
\end{array}
$$


In particular, if we consider a manifold $M$ and we let $r(x)$ be the distance function from a fixed origin $o,(3.29)$ and $v(r) \sim \omega_{m-1} r^{m-1}$ imply that $\chi(r(x))$ has an integrable singularity near $o$ for every $m \geq 2$. Next, we consider the examples of Euclidean and hyperbolic spaces.

EXAmPle 3.14 (Euclidean space). Let $M$ be the Euclidean space $\mathbb{R}^{m}$. Then, $v(r)=\omega_{m-1} r^{m-1}$, so we have to exclude $m=2$ since $\left(\mathrm{V}_{\mathrm{L} 1}\right)$ does not hold. For every $m \geq 3$, a simple computation gives

$$
\chi(r)=\frac{(m-2)^{2}}{4 r^{2}} \quad \text { on } \mathbb{R}^{+} .
$$

Similarly, if $v(r)=\Lambda r^{\alpha}$ for $r \geq r_{0}>0$, where $\Lambda>0$ and $\alpha>1$,

$$
\chi(r)=\frac{(\alpha-1)^{2}}{4 r^{2}} \quad \text { on }\left[r_{0},+\infty\right) .
$$

We mention that a polynomial growth of type $r^{\alpha}$ is the case, for instance, of transient metric trees (see [EFK11]) and some fractal spaces, and that many of the arguments of the next chapters can be rephrased and extended to be applied in these general settings.

EXAmPle 3.15 (The hyperbolic space). Some computations are required for the hyperbolic space $\mathbb{H}_{B}^{m}$ of sectional curvature $-B^{2}<0$. In this case, the volume of geodesic spheres is $v(r)=B^{1-m} \sinh ^{m-1}(B r)$. Set

$$
I_{m}(r)=\int_{r}^{+\infty} \sinh ^{1-m}(B s) \mathrm{d} s, \quad \text { so that } \quad \frac{1}{2 \sqrt{\chi(r)}}=\sinh ^{m-1}(B r) I_{m}(r) .
$$

Denote, for convenience, with $\chi_{m}$ the critical curve of $\mathbb{H}_{B}^{m}$. From the recursive relation

$$
(m-1) I_{m}(r)=\frac{1}{B} \cosh (B r) \sinh ^{-m}(B r)-m I_{m+2}(r),
$$

which can be proved integrating by parts, we deduce

$$
\frac{m-1}{2 \sqrt{\chi_{m}(r)}}=\frac{\operatorname{coth}(B r)}{B}-\frac{1}{\sinh ^{2}(B r)} \frac{m}{2 \sqrt{\chi_{m+2}(r)}} .
$$

Therefore, we can compute the explicit expression of $\chi_{m}$ for every $m$ once we know those of $\chi_{2}$ and $\chi_{3}$. If $m=3$,

$$
I_{3}(r)=B^{-1} \int_{r}^{+\infty}(\operatorname{coth}(B s))^{\prime} \mathrm{d} s=B^{-1}(\operatorname{coth}(B r)-1),
$$

hence

$$
\chi(r)=\frac{B^{2}}{\left(1-e^{-2 B r}\right)^{2}} \quad \text { on } \mathbb{H}_{B}^{3} .
$$

If $m=2$, we change variables according to $\sigma=e^{B s}$ to deduce

$$
I_{2}(r)=\frac{2}{B} \int_{e^{B r}}^{+\infty} \frac{\mathrm{d} \sigma}{\sigma^{2}-1}=\frac{1}{B} \log \left(\frac{e^{B r}+1}{e^{B r}-1}\right)
$$

and thus

$$
\chi(r)=B^{2}\left[2 \sinh (B r) \log \left(\frac{e^{B r}+1}{e^{B r}-1}\right)\right]^{-2} \quad \text { on } \mathbb{H}_{B}^{2}
$$


In what follows, particularly in Chapters 5 and 6 , it will be useful to consider bounds $f$ for $v$ of the following type:

$$
f(r)=\Lambda \exp \left\{a r^{\alpha} \log ^{\beta} r\right\}, \quad \Lambda, a, \alpha>0, \beta \geq 0,
$$

on $I=\mathbb{R}^{+}$or on $I=\left[r_{0},+\infty\right)$. In the easy case $\alpha=1, \beta=0$, that is, $f(r)=$ $\Lambda \exp \{a r\}$, the critical curve is constant:

$$
\chi_{f}(r) \equiv \frac{a^{2}}{4} \quad \text { on } I \text {. }
$$

In the general case, $\chi_{f}(r)$ cannot be explicitly computed in terms of elementary functions, so we concentrate on the asymptotic behaviour as $r \rightarrow+\infty$. Again using De l'Hopital rule

$$
\chi_{f}(r) \sim\left(\frac{a^{2} \alpha^{2}}{4}\right) r^{2(\alpha-1)} \log ^{2 \beta} r \sim\left[\frac{f^{\prime}(r)}{2 f(r)}\right]^{2} \quad \text { as } r \rightarrow+\infty .
$$

Therefore, with the choice (3.33), the critical function $\chi_{f}(r)$ is asymptotic to what we shall call from now on the modified critical function $\widetilde{\chi}_{f}(r)$ :

$$
\tilde{\chi}_{f}(r)=\left[\frac{f^{\prime}(r)}{2 f(r)}\right]^{2} .
$$

As we will stress later, $\chi$ and $\tilde{\chi}$ are deeply related. Here we limit ourselves to observe that, if $f(r)=g(r)^{m-1}$ comes from the Laplacian comparison theorem,

$$
\tilde{\chi}_{f}(r)=\frac{1}{4}\left[(m-1) \frac{g^{\prime}(r)}{g(r)}\right]^{2}
$$

directly depends on a bound for $\Delta r$. The modified critical function, being asymptotic to $\chi_{f}$ when $f$ is of type (3.33), will come in handy in Chapter 6 to control the oscillations of $\left(v z^{\prime}\right)^{\prime}+A v z=0$.

Combining Bishop-Gromov volume comparison theorem and Proposition 3.13, we provide upper and lower bounds at infinity in some useful geometrical situations. This is the content of the next three results. We begin with

TheOREM 3.16 (Upper bounds for $\chi(r)$ on $\mathbb{R}^{+}$). Let $(M,\langle\rangle$,$) be a complete$ manifold of dimension $m \geq 2$ satisfying

$$
\operatorname{Ricc}(\nabla r, \nabla r)(x) \geq-(m-1) B^{2}\left(1+r(x)^{2}\right)^{\alpha / 2} \quad \text { on } M,
$$

for some $B>0$ and $\alpha \geq-2$. Then,

(i) If $\alpha \geq 0, \chi(r) \leq \chi_{f}(r)$ on $\mathbb{R}^{+}$, where

$$
f(r)=B^{1-m} \sinh ^{m-1}\left(\frac{2 B}{2+\alpha}\left[(1+r)^{1+\frac{\alpha}{2}}-1\right]\right),
$$

and

$$
\chi_{f}(r) \sim \frac{B^{2}(m-1)^{2}}{4} r^{\alpha} \quad \text { as } r \rightarrow+\infty .
$$

(ii) If $\alpha \in(-2,0), \chi(r) \leq \chi_{f}(r)$ on $\mathbb{R}^{+}$, where

$$
f(r)=r^{(m-1) / 2}\left[I_{\frac{1}{2+\alpha}}\left(\frac{2 B}{2+\alpha} r^{1+\frac{\alpha}{2}}\right)\right]^{m-1}
$$


and $I_{\nu}(s)$ is the modified Bessel function of order $\nu$. Moreover,

$$
\chi_{f}(r) \sim \frac{B^{2}(m-1)^{2}}{4} r^{\alpha} \quad \text { as } r \rightarrow+\infty .
$$

(iii) If $\alpha=-2$,

$$
\chi(r) \leq \frac{\left(B^{\prime}(m-1)-1\right)^{2}}{4 r^{2}} \text { on } \mathbb{R}^{+}, \text {where } B^{\prime}=\frac{1+\sqrt{1+4 B^{2}}}{2} .
$$

Proof. $(i)$ The function $g(r)=f(r)^{1 /(m-1)}$ solves $(3.26)$ with $G(r)=B^{2}(1+$ $\left.r^{2}\right)^{\alpha / 2}$. Then, by Proposition 3.13 we deduce $\chi \leq \chi_{f}$, where $f=g^{m-1}$. An application of De l'Hopital rule gives, for some explicit $C>0$,

$$
\begin{aligned}
f(r) & \sim C \exp \left(\frac{2 B(m-1)}{2+\alpha}(1+r)^{1+\frac{\alpha}{2}}\right), \\
\int_{r}^{+\infty} \frac{\mathrm{d} s}{f(s)} & \sim C^{-1} \frac{1}{B(m-1)}(1+r)^{-\alpha / 2} \exp \left(-\frac{2 B(m-1)}{2+\alpha}(1+r)^{1+\frac{\alpha}{2}}\right) .
\end{aligned}
$$

The asymptotic behaviour of $\chi_{f}$ follows immediately.

As for $(i i)$, since $I_{\nu}(s), \nu>0$, is a positive solution of the Bessel equation

$$
\begin{aligned}
& s^{2} \frac{\mathrm{d}^{2} I_{\nu}}{\mathrm{d} s^{2}}+s \frac{\mathrm{d} I_{\nu}}{\mathrm{d} s}-\left(s^{2}+\nu^{2}\right) I_{\nu}=0, \\
& I_{\nu}(s)=\sum_{k=0}^{+\infty} \frac{1}{\Gamma(k+1) \Gamma(k+\nu+1)}\left(\frac{x}{2}\right)^{\nu+2 k}
\end{aligned}
$$

(see [Leb72], p.102), a straightforward computation shows that $g(r)=f(r)^{1 /(m-1)}$ is a positive solution of the singular equation $g^{\prime \prime}-B^{2} r^{\alpha} g=0$ with initial condition $g(0)=0, g^{\prime}(0)=C>0$ for some positive constant $C=C(\alpha, B)$. Hence, since $\alpha<0, g$ satisfies

$$
\left\{\begin{array}{l}
g^{\prime \prime}-B^{2}\left(1+r^{2}\right)^{\alpha / 2} g \geq g^{\prime \prime}-B^{2} r^{\alpha} g=0 \\
g(0)=0, \quad g^{\prime}(0)=C>0,
\end{array}\right.
$$

so that $\chi \leq \chi_{\tilde{f}}$, where $\tilde{f}=\left(C^{-1} g\right)^{m-1}$ is proportional to $f$. Since $\chi$ in invariant under multiplication by a positive constant, $\chi \leq \chi_{f}$. Using

$$
I_{\nu}(s)=\frac{e^{s}}{\sqrt{2 \pi s}}(1+o(1)) \quad \text { as } s \rightarrow+\infty
$$

(see [Leb72], p.123) and De l'Hopital rule we deduce, for some explicit $C>0$,

$$
\begin{aligned}
f(r) & \sim C r^{-\frac{(m-1) \alpha}{4}} \exp \left(\frac{2 B(m-1)}{2+\alpha} r^{1+\frac{\alpha}{2}}\right), \\
\int_{r}^{+\infty} \frac{\mathrm{d} s}{f(s)} & \sim C^{-1} \frac{1}{B(m-1)} r^{(m-3) \frac{\alpha}{4}} \exp \left(-\frac{2 B(m-1)}{2+\alpha} r^{1+\frac{\alpha}{2}}\right),
\end{aligned}
$$

thus $\chi_{f} \sim\left[B^{2}(m-1)^{2} / 4\right] r^{\alpha}$ also when $\alpha \in(-2,0)$. It remains to examine $(i i i)$. The function

solves

$$
g(r)=r^{B^{\prime}}, \quad B^{\prime}=\frac{1+\sqrt{1+4 B^{2}}}{2}
$$

$$
\left\{\begin{array}{l}
g^{\prime \prime}-B^{2}\left(1+r^{2}\right)^{-1} g \geq g^{\prime \prime}-B^{2} r^{-2}=0 \\
g(0)=0, \quad g^{\prime}(0)=0 .
\end{array}\right.
$$


Condition $g^{\prime}(0)=0$ requires some care. Let $h$ be the (positive) solution of

$$
\left\{\begin{array}{l}
h^{\prime \prime}-B^{2}\left(1+r^{2}\right)^{-1} h=0 \quad \text { on } \quad \mathbb{R}_{0}^{+}, \\
h(0)=0, \quad h^{\prime}(0)=1 .
\end{array}\right.
$$

Then, $\left(h g^{\prime}-g h^{\prime}\right)^{\prime} \geq 0$ on $\mathbb{R}^{+}$. Since $\left(h g^{\prime}-g h^{\prime}\right)\left(0^{+}\right)=0$, we deduce $h g^{\prime}-g h^{\prime} \geq 0$, hence $g / h$ is increasing. Applying both Propositions 3.12 and 3.13 we get

$$
\chi(r) \leq \chi_{h^{m-1}}(r) \leq \chi_{g^{m-1}}(r)=\frac{\left(B^{\prime}(m-1)-1\right)^{2}}{4 r^{2}} \quad \text { on } \mathbb{R}^{+} .
$$

REMARK 3.17. Observe that, in (iii), the upper bound $\left(B^{\prime}(m-1)-1\right)^{2} / 4 r^{2}$ fails to have the right behaviour (3.29) at $r=0^{+}$. This fact is due to $g^{\prime}(0)=0$ in (3.45).

Next, we consider lower bounds for $\chi(r)$ on negatively curved manifolds.

THEOREM 3.18 (Lower bounds for $\chi(r)$ on $\mathbb{R}^{+}$). Let $(M,\langle\rangle$,$) be a complete$ manifold of dimension $m \geq 2$ satisfying

$$
\operatorname{cut}(o)=\emptyset, \quad K_{\mathrm{rad}}(x) \leq-B^{2}\left(1+r(x)^{2}\right)^{\alpha / 2} \quad \text { on } M,
$$

for some $B>0$ and $\alpha \geq-2$. Then,

(i) If $\alpha \geq 0, \chi(r) \geq \chi_{f}(r)$ on $\mathbb{R}^{+}$, where $f(r)$ is as in (3.38) and satisfies

$$
\chi_{f}(r) \sim \frac{B^{2}(m-1)^{2}}{4} r^{\alpha} \quad \text { as } r \rightarrow+\infty .
$$

(ii) If $\alpha \in(-2,0), \chi(r) \geq \chi_{f}(r)$ on $\mathbb{R}^{+}$, where $f(r)$ is as in (3.37) and satisfies (3.47).

(iii) If $\alpha=-2$,

$$
\chi(r) \geq \frac{\left(B^{\prime}(m-1)-1\right)^{2}}{4(1+r)^{2}} \text { on } \mathbb{R}^{+}, \text {where } B^{\prime}=\frac{1+\sqrt{1+4 B^{2}}}{2} .
$$

Proof. The proof is dual to that of Theorem 3.16. As for $(i)$, since $g(r)=$ $f(r)^{1 /(m-1)}$ solves $g^{\prime \prime}-B^{2} r^{\alpha} g=0$ with initial condition $g(0)=0, g^{\prime}(0)=C(\alpha, B)>$ 0 , when $\alpha \geq 0, g$ satisfies

$$
\left\{\begin{array}{l}
g^{\prime \prime}-B^{2}\left(1+r^{2}\right)^{\alpha / 2} g \leq g^{\prime \prime}-B^{2} r^{\alpha} g=0 \\
g(0)=0, \quad g^{\prime}(0)=C .
\end{array}\right.
$$

By comparison, $\chi \geq \chi_{\widetilde{f}} \equiv \chi_{f}$, where $\tilde{f}=\left(C^{-1} g\right)^{m-1}=C^{1-m} f$.

Case (ii) is identical. It is enough to observe that, when $\alpha \in(-2,0), g(r)=$ $f(r)^{1 /(m-1)}$ solves

$$
\left\{\begin{array}{l}
g^{\prime \prime}-B^{2}\left(1+r^{2}\right)^{\alpha / 2} g \leq g^{\prime \prime}-B^{2}(1+r)^{\alpha} g(r) \leq 0 \\
g(0)=0, \quad g^{\prime}(0)=1
\end{array}\right.
$$

We are left to the almost Euclidean case, that is, $($ iii $)$. Consider $h(r)=(1+r)^{B^{\prime}}$. Then, $h(0)=1, h^{\prime}(0)=0$ and

$$
h^{\prime \prime}(r)=\frac{B^{\prime}\left(B^{\prime}-1\right)}{(1+r)^{2}} h(r)=\frac{B^{2}}{(1+r)^{2}} h(r) \leq \frac{B^{2}}{1+r^{2}} h(r) .
$$


Therefore, if $g$ satisfy

$$
\left\{\begin{array}{l}
g^{\prime \prime}-B^{2}\left(1+r^{2}\right)^{\alpha / 2} g=0 \\
g(0)=0, \quad g^{\prime}(0)=1
\end{array}\right.
$$

$\left(g^{\prime} h-g h^{\prime}\right)^{\prime} \geq 0$ on $\mathbb{R}^{+}$and $\left(g^{\prime} h-g h^{\prime}\right)(0)=1$, hence $(g / h)^{\prime}>0$. This implies that $g / h$ is increasing, and applying Propositions 3.12 and 3.13

$$
\chi(r) \geq \chi_{g^{m-1}}(r) \geq \chi_{h^{m-1}}(r)=\frac{\left(B^{\prime}(m-1)-1\right)^{2}}{4(1+r)^{2}},
$$

which concludes the proof.

REMARK 3.19. If $\alpha=0$ in the above theorem, that is, $K_{\text {rad }}(x) \leq-B^{2}$, we indeed have the simpler lower bound

$$
\chi(r) \geq \chi_{f}(r)>\frac{B^{2}(m-1)^{2}}{4} \quad \text { on } \mathbb{R}^{+} .
$$

To see this, by case (ii) of Theorem 3.18, $\chi \geq \chi_{g^{m-1}}$, where $g=B^{-1} \sinh (B r)$. Therefore, to prove (3.51) it is enough to consider the solution $h(r)=\exp (B r)$ of

$$
\left\{\begin{array}{l}
h^{\prime \prime}-B^{2} h=0, \\
h(0)=1, \quad h^{\prime}(0)=B
\end{array} \quad \text { for which } \quad \chi_{h^{m-1}}(r) \equiv \frac{B^{2}(m-1)^{2}}{4} \text { on } \mathbb{R}^{+} .\right.
$$

Comparing with $g$ (note that $g(0)=0, g^{\prime}(0)=1$ ), by Sturm argument $h / g$ is strictly decreasing, hence by Proposition $3.12 \chi_{g^{m-1}}>\chi_{h^{m-1}}$, as desired.

We now consider upper and lower bounds when the manifold $M$ has possibly non-negative radial sectional curvature. Note that, by the volume comparison theorem, if $K_{\mathrm{rad}} \geq 0$ then $v(r)=\operatorname{vol}\left(\partial B_{r}\right) \leq \omega_{m-1} r^{m-1}$. Hence, the case $m=2$ has to be excluded since $1 / v \notin L^{1}(+\infty)$. The proofs follow the same procedure as those of Theorems 3.16 and 3.18 , so we only sketch them.

TheOREm 3.20 (Upper bounds for $\chi(r)$ on $\mathbb{R}^{+}$). Let $(M,\langle\rangle$,$) be a complete,$ non-compact manifold of dimension $m \geq 3$ satisfying

$$
\operatorname{Ricc}(\nabla r, \nabla r) \geq(m-1) \frac{B^{2}}{(1+r(x))^{2}} \quad \text { on } M,
$$

for some $B \leq 1 / 2$. Then,

(i) If $B<1 / 2$, then $\chi(r) \leq \chi_{f}(r)$ on $\mathbb{R}^{+}$, where

$$
f(r)=\left((1+r)^{B^{\prime \prime}}-(1+r)^{1-B^{\prime \prime}}\right)^{m-1}, \quad B^{\prime \prime}=\frac{1+\sqrt{1-4 B^{2}}}{2},
$$

and

$$
\chi_{f}(r) \sim \frac{\left(B^{\prime \prime}(m-1)-1\right)^{2}}{4 r^{2}} \quad \text { as } r \rightarrow+\infty .
$$

(ii) If $B=1 / 2$, then $\chi(r) \leq \chi_{f}(r)$ on $\mathbb{R}^{+}$, where

$$
f(r)=(1+r)^{\frac{m-1}{2}} \log ^{m-1}(1+r)
$$


satisfies

$$
\chi_{f}(r) \begin{cases}\sim \frac{(m-3)^{2}}{16 r^{2}} & \text { if } m>3 \\ =\frac{1}{4(1+r)^{2} \log ^{2}(1+r)} & \text { if } m=3\end{cases}
$$

Proof. It is enough to compare the critical curve with that of a model manifold $\left(M_{g}, \mathrm{~d} s^{2}\right)$, where $g$ is the explicit solution of the Cauchy problem for the Euler equation described in Remark 1.22. The behaviour of each critical curve can be easily computed. In particular, (3.53) follows from

$$
\int_{r}^{+\infty} \frac{\mathrm{d} s}{g(s)^{m-1}} \begin{cases}\sim \frac{2}{m-3} r^{-\frac{m-3}{2}} \log ^{-(m-1)} r & \text { if } m>3 \\ =\frac{1}{\log (1+r)} & \text { if } m=3 .\end{cases}
$$

Lower bounds can be found by comparing, again, with the solutions of Euler equation. However, for future use, it is more convenient to compare with functions $g$ for which the critical curve is simpler. As we will see in Theorem 4.10, this will enable us to deal also with some border line case for which the sole asymptotic behaviour of the critical curve as $r \rightarrow+\infty$ is not enough to produce a sharp result.

THEOREM 3.21 (Lower bounds for $\chi(r)$ on $\mathbb{R}^{+}$). Let $(M,\langle$,$\rangle ) be a complete,$ non-compact manifold of dimension $m \geq 3$ satisfying

$$
\operatorname{cut}(o)=\emptyset, \quad K_{\mathrm{rad}}(x) \leq \frac{B^{2}}{(1+r(x))^{2}} \quad \text { on } M
$$

for some $B \leq 1 / 2$.

(i) If $B<1 / 2$ or $B=1 / 2$ and $m>3$, then

$$
\chi(r) \geq \frac{\left(B^{\prime \prime}(m-1)-1\right)^{2}}{4 r^{2}} \text { on } \mathbb{R}^{+}, \text {where } B^{\prime \prime}=\frac{1+\sqrt{1-4 B^{2}}}{2} \text {. }
$$

(ii) If $B=1 / 2$ and $m=3$, then

$$
\chi(r) \geq \frac{1}{4(1+r)^{2} \log ^{2}(1+r)} \quad \text { on } \mathbb{R}^{+} .
$$

Proof. In case $(i)$, we consider the function $h(r)=r^{B^{\prime \prime}}$ which solves

$$
\left\{\begin{array}{l}
h^{\prime \prime}+\frac{B^{2}}{(1+r)^{2}} h \leq h^{\prime \prime}+\frac{B^{2}}{r^{2}} h=0 \\
h(0)=0
\end{array}\right.
$$

Note that, in both the cases

$$
B<1 / 2, \quad m \geq 3 \quad \text { and } \quad B=1 / 2, \quad m>3
$$

we have $h^{1-m} \in L^{1}(+\infty)$. Now, if $g$ is the solution of

$$
\left\{\begin{array}{l}
g^{\prime \prime}+\frac{B^{2}}{(1+r)^{2}} g=0 \\
g(0)=0, \quad g^{\prime}(0)=1
\end{array}\right.
$$


by Sturm argument $g / h$ is non-decreasing. By Propositions 3.13 and 3.12,

$$
\chi(r) \geq \chi_{g^{m-1}}(r) \geq \chi_{h^{m-1}}(r)=\frac{\left(B^{\prime \prime}(m-1)-1\right)^{2}}{4 r^{2}} \quad \text { on } \mathbb{R}^{+} .
$$

To show $(i i)$, we compare directly with the solution $g(r)=\sqrt{1+r} \log (1+r)$ of

$$
\left\{\begin{array}{l}
g^{\prime \prime}+\frac{1}{4(1+r)^{2}} g=0 \\
g(0)=0, \quad g^{\prime}(0)=1 .
\end{array}\right.
$$

Define $f(r)=g(r)^{m-1}$. Since $m=3$, by (3.53) $\chi_{g^{m-1}}$ can be explicitly computed and has the expression in (3.57).

To conclude, we consider estimates for $\chi$ when we can only control the Ricci or sectional curvatures in a neighbourhood of $+\infty$, that is, on $\left[r_{0},+\infty\right)$ for some $r_{0}>0$. The principal problem is to construct subsolutions and supersolutions whose initial conditions allow the application of Sturm type arguments. The basic step is the following technical lemma. For the ease of notation, we set

$$
D(t)=\frac{1}{2}\left(\sqrt{t^{2}+4}-t\right) \quad \text { on } \mathbb{R},
$$

and we observe that $D$ is positive, decreasing on $\mathbb{R}$, and such that $D(0)=1$.

LEMMA 3.22. Let $0 \leq H \in C^{1}\left(\left[r_{0},+\infty\right)\right)$, for some $r_{0}>0$. Let $h_{0}, h_{1}$ be fixed positive numbers, and define

$$
\theta_{*}=\liminf _{r \rightarrow+\infty} \frac{H^{\prime}}{2 H^{3 / 2}}, \quad \theta^{*}=\limsup _{r \rightarrow+\infty} \frac{H^{\prime}}{2 H^{3 / 2}} .
$$

(1) Suppose that $\theta_{*}>-\infty$. Let $D_{o}>D\left(\theta_{*}\right)$, and let $\theta<\theta_{*}$ be close enough to $\theta_{*}$ so that $D_{o}>D(\theta)$. Let $r_{1}>r_{0}$ be sufficiently large that

$$
\frac{H^{\prime}}{2 H^{3 / 2}}>\theta \quad \text { on }\left[r_{1},+\infty\right) \text {. }
$$

Let $C>0$ be a positive number satisfying

$$
C \geq \max \left\{h_{0}, \frac{h_{1}}{D_{o} \sqrt{H\left(r_{1}\right)}}\right\} .
$$

Then, the function

$$
h(r)=C\left\{\exp \left(D_{o} \int_{r_{1}}^{r} \sqrt{H(s)} \mathrm{d} s\right)-1\right\}+h_{0}
$$

satisfies

$$
\left\{\begin{array}{l}
h^{\prime \prime}-H h \geq 0 \quad \text { on }\left[r_{1},+\infty\right) \\
h\left(r_{1}\right)=h_{0}, \quad h^{\prime}\left(r_{1}\right) \geq h_{1} .
\end{array}\right.
$$

(2) Suppose that $\theta^{*}<+\infty$. Let $0<D_{o}<D\left(\theta^{*}\right)$, and let $\theta>\theta^{*}$ be close enough to $\theta^{*}$ so that $D_{o}<D(\theta)$. Let $r_{1}>r_{0}$ be sufficiently large that

$$
\frac{H^{\prime}}{2 H^{3 / 2}}<\theta \quad \text { on }\left[r_{1},+\infty\right) \text {. }
$$

Let $C>0$ be a positive number satisfying

$$
C \leq \min \left\{h_{0}, \frac{h_{1}}{D_{o} \sqrt{H\left(r_{1}\right)}}\right\} .
$$


Then, the function

$$
h(r)=C\left\{\exp \left(D_{o} \int_{r_{1}}^{r} \sqrt{H(s)} \mathrm{d} s\right)-1\right\}+h_{0}
$$

satisfies

$$
\left\{\begin{array}{l}
h^{\prime \prime}-H h \leq 0 \quad \text { on }\left[r_{1},+\infty\right) \\
h\left(r_{1}\right)=h_{0}, \quad h^{\prime}\left(r_{1}\right) \leq h_{1} .
\end{array}\right.
$$

Proof. We prove item (1), the other case being analogous. By property (3.62), $h(r)$ defined in (3.63) satisfies $h\left(r_{1}\right)=h_{0}, h^{\prime}\left(r_{1}\right) \geq h_{1}$. Moreover,

$$
h^{\prime \prime}-H h=C \exp \left(D_{o} \int \sqrt{H}\right) D_{o} H\left[D_{o}-\frac{1}{D_{o}}+\frac{H^{\prime}}{2 H^{3 / 2}}\right]+H\left(C-h_{0}\right) .
$$

Using (3.61), $D_{o}>D(\theta)$ and the definition of $D(t)$, on $\left[r_{1},+\infty\right)$ the term between square brackets is bounded as follows:

$$
D_{o}-\frac{1}{D_{o}}+\frac{H^{\prime}}{2 H^{3 / 2}}>D_{o}-\frac{1}{D_{o}}+\theta>0 .
$$

Since, by (3.62), $C \geq h_{0}$, inserting into (3.64) we obtain $h^{\prime \prime}-H h \geq 0$, as desired.

In the next Proposition, we apply the above lemma to the particular case $H(r)=B^{2} r^{\alpha}$, together with the comparisons we have described in this section, to derive upper and lower estimates for the critical function. In what follows, to simplify the writing, we introduce the symbol $f \lesssim g$ as $r \rightarrow+\infty$ to mean that $\lim \sup _{r \rightarrow+\infty}(f / g) \leq 1$.

Proposition 3.23 (Bounds for $\chi(r)$ near $+\infty)$. Let $(M,\langle\rangle$,$) be a non-compact,$ complete Riemannian manifold, and let $r(x)$ be the distance function from a reference origin $o$.

(i) suppose that $\operatorname{Ricc}(\nabla r, \nabla r) \geq-(m-1) B^{2} r^{\alpha}$ on $M \backslash B_{r_{0}}$, for some $r_{0}>0$ and for some $B>0, \alpha \geq-2$. Then,

$$
\chi(r) \lesssim \begin{cases}\frac{B^{2}(m-1)^{2}}{4} r^{\alpha} & \text { as } r \rightarrow+\infty, \quad \text { if } \alpha>-2 ; \\ \frac{\left(B^{\prime}(m-1)-1\right)^{2}}{4 r^{2}} & \text { as } r \rightarrow+\infty, \quad \text { if } \alpha=-2 .\end{cases}
$$

Where $B^{\prime}=\frac{1}{2}\left(1+\sqrt{1+4 B^{2}}\right)$.

(ii) suppose that $o$ is a pole and that the radial sectional curvatures of $M$ satisfy $K_{\mathrm{rad}}(x) \leq K(r(x))$, where

$$
0 \leq K \in L^{1}(+\infty), \quad r \int_{r}^{+\infty} K(\sigma) \mathrm{d} \sigma \leq \frac{1}{4} \quad \text { on } \mathbb{R}^{+} .
$$

Moreover, assume that $K_{\mathrm{rad}} \leq-B^{2} r^{\alpha}$ on $M \backslash B_{r_{0}}$, for some $r_{0}>0$ and for some $B>0, \alpha>-2$. Then,

$$
\chi(r) \gtrsim \begin{cases}\frac{B^{2}(m-1)^{2}}{4} r^{\alpha} & \text { as } r \rightarrow+\infty, \quad \text { if } \alpha>-2 ; \\ \frac{\left(B^{\prime}(m-1)-1\right)^{2}}{4 r^{2}} & \text { as } r \rightarrow+\infty, \quad \text { if } \alpha=-2 .\end{cases}
$$

Where $B^{\prime}=\frac{1}{2}\left(1+\sqrt{1+4 B^{2}}\right)$. 
In particular, if o is a pole, $K_{\mathrm{rad}}(x) \leq K(r(x))$ for some $K$ satisfying (3.65), and $K_{\mathrm{rad}} \sim-B^{2} r^{\alpha}$ as $r \rightarrow+\infty$, then

$$
\chi(r) \sim \begin{cases}\frac{B^{2}(m-1)^{2}}{4} r^{\alpha} & \text { as } r \rightarrow+\infty, \quad \text { if } \alpha>-2 ; \\ \frac{\left(B^{\prime}(m-1)-1\right)^{2}}{4 r^{2}} & \text { as } r \rightarrow+\infty, \quad \text { if } \alpha=-2 .\end{cases}
$$

Proof. $(i)$. First, we extend the function $B^{2} r^{\alpha}$ continuously on $\left[0, r_{0}\right]$ to a non-negative function $G(r)$ for which

$$
\operatorname{Ricc}(\nabla r, \nabla r) \geq-(m-1) G(r) \quad \text { on } \mathbb{R}^{+} .
$$

By Proposition 1.21, the solution $g$ of

$$
\left\{\begin{array}{l}
g^{\prime \prime}-G g=0 \\
g(0)=0, \quad g^{\prime}(0)=1
\end{array}\right.
$$

is positive and increasing on $\mathbb{R}^{+}$. Furthermore, by Proposition $3.13, \chi \leq \chi_{g^{m-1}}$ on $\mathbb{R}^{+}$. To apply Lemma 3.22 , define $H(r)=B^{2} r^{\alpha}$ and note that

$$
\frac{H^{\prime}}{2 H^{3 / 2}}=\frac{\alpha}{2 B} r^{-\frac{\alpha}{2}-1} \begin{cases}\rightarrow 0 \text { as } r \rightarrow+\infty, & \text { if } \alpha>-2 \\ =-1 / B & \text { if } \alpha=-2\end{cases}
$$

Thus

$$
\begin{array}{lll}
\theta_{*}=\theta^{*}=0, & D\left(\theta_{*}\right)=D\left(\theta^{*}\right)=1 & \text { if } \alpha>-2 \\
\theta_{*}=\theta^{*}=-1 / B & D\left(\theta_{*}\right)=D\left(\theta^{*}\right)=\frac{1}{2 B}\left(1+\sqrt{1+4 B^{2}}\right)=\frac{B^{\prime}}{B} & \text { if } \alpha=-2 .
\end{array}
$$

We choose $D>D\left(\theta_{*}\right), \theta<\theta_{*}$, and $r_{1}>r_{0}$ according to item (1) of Lemma 3.22, and we consider the initial conditions $h_{0}=g\left(r_{1}\right), h_{1}=g^{\prime}\left(r_{1}\right)$. Note that, since $g$ is positive and increasing, $h_{0}, h_{1}>0$. Then, for every $D>1$, by the assumption

$$
\operatorname{Ricc}(\nabla r, \nabla r) \geq-(m-1) B^{2} r^{\alpha}=-(m-1) G(r)=-(m-1) H(r),
$$

the function $h(r)$ in (3.63) is a supersolution of $(3.66)$ on $\left[r_{1},+\infty\right)$ and satisfies

$h\left(r_{1}\right)=g\left(r_{1}\right), h^{\prime}\left(r_{1}\right) \geq g^{\prime}\left(r_{1}\right), \quad h(r) \sim \widehat{C} \begin{cases}\exp \left\{D \frac{2 B}{2+\alpha} r^{\frac{\alpha}{2}+1}\right\} & \text { if } \alpha>-2 ; \\ r^{D B} & \text { if } \alpha=-2,\end{cases}$

for some $\widehat{C}>0$. Then, by Sturm argument $g / h$ is decreasing, hence by Proposition 3.12

$$
\chi_{g^{m-1}} \leq \chi_{h^{m-1}} \sim \begin{cases}D^{2} \frac{B^{2}(m-1)^{2}}{4} r^{\alpha} & \text { as } r \rightarrow+\infty, \text { if } \alpha>-2 ; \\ \frac{(D B(m-1)-1)^{2}}{4 r^{2}} & \text { as } r \rightarrow+\infty, \text { if } \alpha=-2 .\end{cases}
$$

Letting $D \downarrow D\left(\theta^{*}\right)$ we get the desired bounds.

Case (ii) can be proved similarly. Indeed, let $G(r)$ be a continuous function satisfying

$K_{\text {rad }}(x) \leq-G(r(x))$ on $M, \quad G(r)=B^{2} r^{\alpha}$ on $\left[r_{0},+\infty\right), \quad-G(r) \leq K(r)$ on $\mathbb{R}^{+}$.

By Proposition 1.21, the assumptions (3.65) on $K(r)$ ensure that the solution $g$ of (3.66) is positive and increasing on $\mathbb{R}^{+}$. This is essential to apply item (2) of 
Lemma 3.22 and to conclude along the same lines as for $(i)$. The last part of the proposition follows from $(i),(i i)$ and a simple limit argument.

Corollary 3.24. Let $(M,\langle\rangle$,$) be a non-compact, complete manifold with a$ pole $o$ and radial sectional curvature satisfying

$$
K_{\text {rad }}(x) \leq-G(r(x)) \quad \text { on } M \backslash\{o\},
$$

for some $G \in C^{0}\left(\mathbb{R}_{0}^{+}\right)$such that

$$
G_{-} \in L^{1}(+\infty), \quad r \int_{r}^{+\infty} G_{-}(\sigma) \mathrm{d} \sigma \leq \frac{1}{4} \quad \text { on } \quad \mathbb{R}^{+} .
$$

Let $g$ be the solution of

$$
\left\{\begin{array}{l}
g^{\prime \prime}-G g=0 \\
g(0)=0, \quad g^{\prime}(0)=1 .
\end{array}\right.
$$

Suppose that $G(r) \rightarrow+\infty$ as $r \rightarrow+\infty$. Then, both $\chi_{g^{m-1}}(r)$ and $\chi(r)$ diverge as $r \rightarrow+\infty$.

Proof. Clearly, by Proposition 3.13 it is enough to prove that $\chi_{g^{m-1}}(r) \rightarrow+\infty$ as $r \rightarrow+\infty$. We choose any $B>0$, and we let $r_{0}$ be such that $G(r) \geq B^{2}$ on $\left[r_{0},+\infty\right)$. Then, we apply item $(i i)$ of Proposition 3.23 to the model manifold $\left(M_{g}, \mathrm{~d} s^{2}\right)$ with metric, in polar coordinates, $\mathrm{d} s^{2}=\mathrm{d} r^{2}+g(r)^{2} \mathrm{~d} \theta^{2}$, to deduce

$$
\liminf _{r \rightarrow+\infty} \chi_{g^{m-1}}(r) \geq \frac{B^{2}(m-1)^{2}}{4} .
$$

The desired conclusion follows letting $B \rightarrow+\infty$.

Corollary 3.25. Let $(M,\langle\rangle$,$) be a non-compact, complete manifold with$ radial Ricci curvature satisfying

$$
\operatorname{Ricc}(\nabla r, \nabla r) \geq-(m-1) G(r(x)) \quad \text { on } \quad M \backslash\{o\}
$$

for some $G \in C^{0}\left(\mathbb{R}_{0}^{+}\right), G \geq 0$ such that $G(r) \rightarrow 0$ as $r \rightarrow+\infty$. Let $g$ be a solution of

$$
\left\{\begin{array}{l}
g^{\prime \prime}-G g=0 \\
g(0)=0, \quad g^{\prime}(0)=1 .
\end{array}\right.
$$

Then, both $\chi_{g^{m-1}}(r)$ and $\chi(r)$ tend to zero as $r \rightarrow+\infty$.

PRoOF. The proof is dual to that of Corollary 3.24 and follows from item $(i)$ of Proposition 3.23. We leave the details to the interested reader. 


\section{CHAPTER 4}

\section{Below the critical curve}

In this Chapter, we analyze some consequences of pointwise comparisons between $A(r)$ and $\chi(r)$. In particular, we concentrate on the case $A(r) \leq \chi(r)$, and we provide constancy of the sign of a solution $z$ of (3.1) and estimates on its asymptotic behaviour at infinity. The results so obtained are then applied to the study of geometric problems such as the index of Schrödinger type operators and related uncertainty principle lemmas, and uniqueness of positive solutions of Yamabe-type equations on complete manifolds

\subsection{Positivity and estimates from below}

In this section we prove the main ODE result reported in Theorem 4.1 below and we subsequently prove its sharpness. We also discuss some comparisons with previous results. In the various assumptions we keep the notation of Chapter 3.

Theorem 4.1. Assume (A1), (V1), ( $\left.\mathrm{V}_{\mathrm{L} 1}\right)$ and that

$$
\frac{1}{2 \sqrt{\chi(r)}}=v(r) \int_{r}^{+\infty} \frac{\mathrm{d} s}{v(s)} \longrightarrow 0 \quad \text { as } r \rightarrow 0 .
$$

Furthermore, suppose that

$$
A(r) \leq k \chi(r) \quad \text { on } \mathbb{R}_{0}^{+} \text {, for some } k \in(-\infty, 1] .
$$

Then, every solution $z(r) \in \operatorname{Lip}_{\text {loc }}\left(\mathbb{R}_{0}^{+}\right)$of

$$
\left\{\begin{array}{l}
\left(v(r) z^{\prime}(r)\right)^{\prime}+A(r) v(r) z(r)=0 \quad \text { on } \mathbb{R}^{+} \\
z(0)=z_{0}>0
\end{array}\right.
$$

is positive on $\mathbb{R}_{0}^{+}$and there exist $r_{1}>0$ sufficiently large and a constant $C=$ $C\left(r_{1}\right)>0$ such that

$$
\begin{array}{ll}
z(r) \geq-C \sqrt{\int_{r}^{+\infty} \frac{\mathrm{d} s}{v(s)}} \log \int_{r}^{+\infty} \frac{\mathrm{d} s}{v(s)} & \text { if } k=1 ; \\
z(r) \geq C\left[\int_{r}^{+\infty} \frac{\mathrm{d} s}{v(s)}\right]^{(1-\sqrt{1-k}) / 2} & \text { if } k \in(-\infty, 1) .
\end{array}
$$

on $\left[r_{1},+\infty\right)$. In particular if $v(r) \leq f(r)$ on $\left[r_{1},+\infty\right)$, and $k \in[0,1]$, then there exists $r_{2} \geq r_{1}$ such that

$$
\begin{array}{ll}
z(r) \geq-C \sqrt{\int_{r}^{+\infty} \frac{\mathrm{d} s}{f(s)}} \log \int_{r}^{+\infty} \frac{\mathrm{d} s}{f(s)} & \text { if } k=1 \\
z(r) \geq C\left[\int_{r}^{+\infty} \frac{\mathrm{d} s}{f(s)}\right]^{(1-\sqrt{1-k}) / 2} & \text { if } k \in[0,1) .
\end{array}
$$


on $\left[r_{2},+\infty\right)$.

Proof. The idea of the proof is quite simple. Using $(\mathrm{V} 1)$ and $\left(\mathrm{V}_{\mathrm{L} 1}\right)$ we define

$$
t=t(r)=-\frac{1}{2} \log \int_{r}^{+\infty} \frac{\mathrm{d} s}{v(s)}
$$

and we observe that $t: \mathbb{R}_{0}^{+} \rightarrow I=\left[t_{0},+\infty\right)$ is an increasing bijection, where $t_{0} \in \mathbb{R}$ or $t_{0}=-\infty$ according to whether $1 / v \in L^{1}\left(0^{+}\right)$or not. Indeed,

$$
t^{\prime}(r)=\sqrt{\chi(r)}>0
$$

thus, letting $r(t)$ denote the inverse function of $t(r)$ and indicating differentiation with respect to $t$ with a dot,

$$
\dot{r}(t)=\frac{1}{\sqrt{\chi(r(t))}} .
$$

Next, for a solution $z$ of (4.3), we set

$$
\beta(t)=e^{t} z(r(t)) ;
$$

clearly $\beta: I \rightarrow \mathbb{R}$ and $\beta \in \operatorname{Lip}_{\text {loc }}(I)$. A simple computation using (4.8) gives

$$
\dot{\beta}(t)=e^{t}\left\{\frac{z^{\prime}(r(t))}{\sqrt{\chi(r(t))}}+z(r(t))\right\} .
$$

Using the definition (3.21) of the critical curve, (4.3) and our assumptions it is easy to see that the RHS of (4.10) is in $\operatorname{Lip}_{\text {loc }}$. We can therefore differentiate again and use (4.3) to deduce

$$
\ddot{\beta}(t)=\left\{1-\frac{A(r(t))}{\chi(r(t))}\right\} \beta(t) .
$$

Since $z_{0}>0$, there exists $\delta>0$ such that $z(r)>0$ on $[0, \delta)$. Furthermore $t\left(0^{+}\right)=$ $t_{0} \geq-\infty$, hence there exists a neighbourhood of $t_{0}$ where $\beta(t)>0$. Since $z \in$ $\operatorname{Lip}_{\text {loc }}\left(\mathbb{R}_{0}^{+}\right)$, by (4.1) it holds $z^{\prime}(r) / \sqrt{\chi(r)} \rightarrow 0$ as $r \rightarrow 0^{+}$, hence

$$
\beta\left(t_{0}^{+}\right)=\dot{\beta}\left(t_{0}^{+}\right)=z_{0} \exp \left\{t_{0}^{+}\right\} \geq 0,
$$

with the strict inequality if $t_{0}>-\infty$. Because of (4.11) and (4.2), $\ddot{\beta} \geq 0$ so that $\beta>0$ on $I$ and, because of (4.9), this shows that $z>0$ on $\mathbb{R}_{0}^{+}$. Next, we fix $t_{1} \in I$ in such a way that $\beta\left(t_{1}\right)>0, \dot{\beta}\left(t_{1}\right)>0$. Integrating $\dot{\beta}$ on $\left[t_{1}, t\right]$ and using the convexity of $\beta$ we deduce

$$
\beta(t)=\beta\left(t_{1}\right)+\int_{t_{1}}^{t} \dot{\beta} \mathrm{d} s \geq \beta\left(t_{1}\right)+\left(t-t_{1}\right) \dot{\beta}\left(t_{1}\right) \geq C t
$$

for some constant $C=C\left(t_{1}\right)>0$. Going back to $z(r)$ using (4.6) and (4.9), having set $r_{1}=r\left(t_{1}\right)$ we have the first of (4.4). To show the validity of the first of (4.5) simply observe that the function $h(x)=\sqrt{x} \log x$ is increasing on $\left(0, e^{-2}\right)$ and use $v \leq f$. When $k<1$, estimates can be improved as in the second inequalities appearing in (4.4), (4.5). Indeed, from (4.11), $\ddot{\beta} \geq(1-k) \beta$ on $\left[t_{1},+\infty\right)$ and, comparing with the solution $\gamma$ of $\ddot{\gamma}=(1-k) \gamma$ with the same initial data of $\beta$, we find

$$
\beta(t) \geq C \exp \{t \sqrt{1-k}\} \quad \text { for some } C>0 .
$$


The second estimates in (4.4) and (4.5) follow from (4.6) and (4.9) as before. Note that, in $(4.5)$, the restriction $k \in[0,1]$ is necessary since, for $k<0$, the exponent $(1-\sqrt{1-k}) / 2$ is negative.

REMARK 4.2. In virtue of Remark 3.11, condition (4.1) is met provided, for instance, $v(r)$ is non-decreasing in a neighbourhood of zero.

REMARK 4.3. The proof of Theorem 4.1 can be repeated verbatim to prove both the positivity and the lower bound for the Lip loc solution of

$$
\left\{\begin{array}{l}
\left(v(r) z^{\prime}(r)\right)^{\prime}+A(r) v(r) z(r)=0 \quad \text { on }\left[r_{0},+\infty\right) \\
z\left(r_{0}\right)=z_{0}>0, \quad v\left(r_{0}\right) z^{\prime}\left(r_{0}\right)=0 .
\end{array}\right.
$$

whenever $A(r) \leq k \chi(r)$ on $\left[r_{0},+\infty\right), k \leq 1$. More generally, the same holds for every nonzero solution on $\left[r_{0},+\infty\right)$ whose initial data at $r_{0}$ satisfy

$$
z\left(r_{0}\right)>0, \quad \frac{z^{\prime}\left(r_{0}\right)}{\sqrt{\chi\left(r_{0}\right)}}+z\left(r_{0}\right)>0,
$$

as one can argue from (4.9) and (4.10).

As an application of Theorem 4.1 and Remark 4.3, we state the following

Corollary 4.4. Assume (A1), (V1), ( $\left.\mathrm{V}_{\mathrm{L} 1}\right)$ and $A \leq \chi$ on $\left[r_{0},+\infty\right)$, for some $r_{0}>0$. Let $r_{1}>0$. Then, every nonzero solution $z(r) \in \operatorname{Lip}_{\text {loc }}\left(\left[r_{1},+\infty\right)\right)$ of

$$
\left\{\begin{array}{l}
\left(v(r) z^{\prime}(r)\right)^{\prime}+A(r) v(r) z(r)=0 \quad \text { on }\left[r_{1},+\infty\right) \\
z\left(r_{1}\right)=z_{1} \in \mathbb{R}
\end{array}\right.
$$

is nonoscillatory.

Proof. Suppose by contradiction that $z(r)$ oscillates; then, there exists a point $r_{2}>\max \left\{r_{0}, r_{1}\right\}$ such that $z\left(r_{2}\right)>0$ and $v\left(r_{2}\right) z^{\prime}\left(r_{2}\right)>0$, for otherwise it would be easy to deduce that $z \equiv 0$. Hence, (4.14) is met with $r_{2}$ replacing $r_{0}$, and according to Remark $4.3, z>0$ on $\left[r_{2},+\infty\right)$, contradiction.

To put the above corollary into perspective, we shall compare it with the existing literature. For instance, R. Moore [Moo55] has extensively studied the equation $\left(v z^{\prime}\right)^{\prime}+A v z=0$, adapting and improving a number of previous criteria. In particular, he proves the following

Theorem 4.5 ([Moo55], Theorem 6). Assume (A1), (V1), $\left(\mathrm{V}_{\mathrm{L} 1}\right)$ on $[R,+\infty)$, and set

$$
H(r)=\left(\int_{r}^{+\infty} \frac{\mathrm{d} s}{v(s)}\right)\left(\int_{R}^{r} A(s) v(s) \mathrm{d} s\right) .
$$

Then, a solution of $\left(v z^{\prime}\right)^{\prime}+A v z=0$ is nonoscillatory provided that there exists some $k \in \mathbb{R}$ such that

$$
-k-\sqrt{k} \leq H(r) \leq-k+\sqrt{k} \leq \frac{1}{4} \quad \text { for } r \text { sufficiently large. }
$$

In particular, $z$ is nonoscillatory whenever

$$
\limsup _{r \rightarrow+\infty}\left|\int_{R}^{r} A(s) v(s) \mathrm{d} s\right|<+\infty .
$$


To relate the two criteria, suppose that $A \leq \chi$. Without loss of generality, we can assume that $A \geq 0$. Indeed, if a solution $z$ of $\left(v z^{\prime}\right)^{\prime}+A_{+} v z=0$ is nonoscillatory, where $A_{+}=\max \{A, 0\}$, then by Sturm arguments (see Theorem 1.9 and Remark 3.4) each solution $z$ of $\left(v z^{\prime}\right)^{\prime}+A v z=0$ is nonoscillatory. From the definition (3.21) we get

$$
\int_{R}^{r} A(s) v(s) \mathrm{d} s \leq \int_{R}^{r} \chi(s) v(s) \mathrm{d} s=\left.\frac{1}{4}\left(\int_{s}^{+\infty} \frac{\mathrm{d} \tau}{v(\tau)}\right)^{-1}\right|_{R} ^{r},
$$

hence by (3.22)

$$
H(r) \leq \frac{1}{4}-\frac{1}{4}\left\{\int_{r}^{+\infty} \frac{\mathrm{d} s}{v(s)} / \int_{R}^{+\infty} \frac{\mathrm{d} s}{v(s)}\right\} \uparrow \frac{1}{4} \quad \text { as } r \rightarrow+\infty .
$$

Therefore, since $A \geq 0$, choosing as $k$ each of the (positive) roots of $k+\sqrt{k}=1 / 4$ condition (4.16) is met. Hence, Moore criterion is more general than Corollary 4.4. However, this latter may be of independent interest for its simplicity. Moreover, as we will see later on, it will be a key step to improve other nonoscillation criteria. In particular, see Section 5.7. The reader be warned that, although by Sturm arguments the negative part of $A$ helps the nonoscillatory behaviour of $z$, in general the lower bound $-k-\sqrt{k}$ for $H$ cannot be removed. Counterexamples, such as Example 2 in [Moo55], are related to fast oscillations of the potential $A$. In this respect we stress that, differently from the requirement $A \leq \chi$, the integral condition (4.16) is not automatically preserved when applying Sturm arguments.

REMARK 4.6. It seems that in the literature a systematic use of the change of variables (4.6) to study (4.3) has not been considered. However, we mention that in $[\mathbf{M o o 5 5}]$ the author somehow exploited it at the end of the proof of Theorem 17 .

When $f(r)$ has the expression (3.33), estimate (4.5) for $k=1$ has the following behaviour at infinity:

$$
-\sqrt{\int_{r}^{+\infty} \frac{\mathrm{d} s}{f(s)}} \log \int_{r}^{+\infty} \frac{\mathrm{d} s}{f(s)} \asymp \exp \left\{-\frac{a}{2} r^{\alpha} \log ^{\beta} r\right\} r^{\frac{\alpha+1}{2}} \log ^{\frac{\beta}{2}} r
$$

while if $f$ is of polynomial type, namely $f(r)=\Lambda r^{\alpha}, \alpha>1, \Lambda>0$, we get

$$
-\sqrt{\int_{r}^{+\infty} \frac{\mathrm{d} s}{f(s)}} \log \int_{r}^{+\infty} \frac{\mathrm{d} s}{f(s)} \asymp r^{-\frac{\alpha-1}{2}} \log r .
$$

Despite of its simplicity, Theorem 4.1 enables us to produce estimates from below for linear ODE of the type (4.3) in a sharp and considerably easy way. In the literature, only partial results are known, see for instance [BR97] and [BRS98b]. In these papers much effort has been done to prove positive lower bounds for solutions of

$$
\left\{\begin{array}{l}
z^{\prime \prime}+(m-1) \frac{g^{\prime}}{g} z^{\prime}+A z=0 \quad \text { on } \mathbb{R}^{+}, \\
z\left(0^{+}\right)=z_{0}>0, \quad z^{\prime}\left(0^{+}\right)=0 .
\end{array}\right.
$$

However, both the lack of an explicit critical curve for general $g$ and the tricky, but somewhat involved, techniques used, have forced the authors to consider only the cases $g(r)=r$ (Euclidean setting) and $g(r)=B^{-1} \sinh (B r)$ (for $\left.\mathbb{H}_{B}^{m}\right)$. In both cases, we stress that the estimates at infinity obtained by the authors (Theorems 
2.5 and 3.2 of [BR97]) are the same as those given by (4.17), (4.18).

Next result is somewhat dual to Theorem 4.1, and shows its sharpness.

Proposition 4.7. Assume (A1), (V1), ( $\left.\mathrm{V}_{\mathrm{L} 1}\right),(4.1)$ and

$$
A(r) \geq k \chi(r) \quad \text { on }\left[r_{0},+\infty\right) \text {, }
$$

for some $r_{0}>0$ and $k \in(-\infty, 1]$. If

$$
\left\{\begin{array}{l}
\left(v(r) z^{\prime}(r)\right)^{\prime}+A(r) v(r) z(r)=0 \quad \text { on }\left[r_{0},+\infty\right) \\
z\left(r_{0}\right)=z_{0}>0 .
\end{array}\right.
$$

admits a solution $z(r) \in \operatorname{Lip}_{\text {loc }}\left(\left[r_{0},+\infty\right)\right)$ which is positive on $\left[r_{0},+\infty\right)$, then necessarily, for some positive constant $C\left(r_{1}\right)$,

$$
\begin{array}{ll}
z(r) \leq-C \sqrt{\int_{r}^{+\infty} \frac{\mathrm{d} s}{v(s)}} \log \int_{r}^{+\infty} \frac{\mathrm{d} s}{v(s)} & \text { if } k=1 \\
z(r) \leq C\left[\int_{r}^{+\infty} \frac{\mathrm{d} s}{v(s)}\right]^{(1-\sqrt{1-k}) / 2} & \text { if } k \in(-\infty, 1) .
\end{array}
$$

Proof. If $A(r) \geq k \chi(r)$, the function $\beta(t)$ introduced in the proof of Theorem 4.1 satisfies $\ddot{\beta} \leq(1-k) \beta, \beta\left(t_{0}\right)>0$, where $t_{0}=t\left(r_{0}\right)$. Therefore, $\beta$ is below some straight line $(k=1)$ or some exponential curve $(k<1)$ at $+\infty$, and estimate $(4.20)$ follows at once by using (4.6), (4.9).

Next, we apply Theorem 4.1 to the study of the equation $g^{\prime \prime}-G g=0$, with initial conditions $g(0)=0, g^{\prime}(0)>0$, and we prove Proposition 1.21.

Remark 4.8 (Proof of Proposition 1.21). By Sturm argument, $g^{\prime} / g \geq \widetilde{g}^{\prime} / \widetilde{g}$ and $g \geq \widetilde{g}$ on $\mathbb{R}^{+}$, where $\widetilde{g}$ solves the same Cauchy problem of $g$ with $-G_{-}$replacing $G$. Hence, without loss of generality we can assume $G \leq 0$. Furthermore, again by Sturm argument, we can assume that $g$ satisfies $g^{\prime \prime}-G g=0$, in place of the inequality. From the initial conditions, we can choose $\varepsilon>0$ small enough that $g, g^{\prime}>0$ on $(0, \varepsilon]$. We are going to show that $g, g^{\prime}>0$ on $[\varepsilon,+\infty)$. Towards this aim we define

$$
\omega(s)=\frac{1}{4 s}-\int_{s}^{+\infty} G(\sigma) \mathrm{d} \sigma \quad \text { on } \mathbb{R}^{+} .
$$

Then, by (1.52) and $G \leq 0$, we have $\omega>0$ and $\omega$ satisfies $\omega^{\prime}+\omega^{2} \leq G$ on $\mathbb{R}^{+}$. Since $\omega-1 /(4 s)$ is bounded in a neighbourhood of zero,

$$
h(s)=s^{1 / 4} \exp \left\{-\int_{0}^{s}\left(\int_{\sigma}^{+\infty} G(\tau) \mathrm{d} \tau\right) \mathrm{d} \sigma\right\}=s^{1 / 4} \exp \left\{\int_{0}^{s}\left(\omega(\sigma)-\frac{1}{4 \sigma}\right) \mathrm{d} \sigma\right\}
$$

is well defined and positive on $\mathbb{R}^{+}$. A computation shows that

$$
h^{\prime}=h \omega>0, \quad h^{\prime \prime}-G h \leq 0 \quad \text { on } \quad \mathbb{R}^{+}, \quad h^{\prime}(s)=\frac{1}{4} s^{-\frac{3}{4}}+o(1) \quad \text { as } \quad s \rightarrow 0^{+} .
$$

Comparing with $g$, we deduce $\left(g^{\prime} h-g h^{\prime}\right)^{\prime} \geq 0$. Since $g(s) \sim s$ as $s \rightarrow 0^{+}$we get $\left(g^{\prime} h-g h^{\prime}\right)\left(0^{+}\right)=0^{+}$, thus $g^{\prime} / g \geq h^{\prime} / h>0$ on $\mathbb{R}^{+}$. The quotient $g / h$ is therefore increasing, and integrating on $[\varepsilon, s]$ we deduce

$$
g(s) \geq h(s) \frac{g(\varepsilon)}{h(\varepsilon)}>0 \quad \text { on }[\varepsilon,+\infty),
$$


which proves that $g>0$ on $\mathbb{R}^{+}$. Consequently, $g^{\prime} \geq h^{\prime} g / h>0$ on $\mathbb{R}^{+}$. To prove the final part of the proposition, it is enough to apply first the change of variables in Proposition 3.10, and then Theorem 4.1. It is easy to see that $A(r) \leq \chi(r)$ is equivalent to (1.54), and that the lower bound (4.4) is of order $\sqrt{s} \log s$ at infinity.

EXAMPLE 4.9. Further understanding is provided by the following examples, which serve the purpose to introduce some conditions related to Chapter 5 .

(1). Equation (4.11) suggests the application of classical oscillation criteria, for example Hille-Nehari Theorem 2.7, to ensure that $z(r)$ is oscillatory (hence, a posteriori, that it has a first zero). Indeed, by (4.9) $\beta(t)$ oscillates if and only if so does $z(r)$. Oscillation of $\beta$ is guaranteed whenever the potential in (4.11) is eventually non-negative, that is, when

$$
A(r) \geq \chi(r) \quad \text { on }[R,+\infty)
$$

and provided

$$
\liminf _{t \rightarrow+\infty} t \int_{t}^{+\infty}\left(\frac{A(r(\tau))}{\chi(r(\tau))}-1\right) \mathrm{d} \tau>\frac{1}{4}
$$

that is, under $\left(\mathrm{V}_{\mathrm{L} 1}\right)$, changing variables according to (4.6) and (4.7),

$$
\liminf _{r \rightarrow+\infty}\left[-\frac{1}{2} \log \int_{r}^{+\infty} \frac{\mathrm{d} s}{v(s)}\right] \int_{r}^{+\infty}\left(\frac{A(s)-\chi(s)}{\sqrt{\chi(s)}}\right) \mathrm{d} s>\frac{1}{4} .
$$

This latter is equivalent, by (3.22), to

$$
\liminf _{r \rightarrow+\infty} \int_{R}^{r} \sqrt{\chi(s)} \mathrm{d} s \int_{r}^{+\infty}\left(\frac{A(s)-\chi(s)}{\sqrt{\chi(s)}}\right) \mathrm{d} s>\frac{1}{4} .
$$

On the other hand, again by Hille-Nehari theorem, $z(r)$ is nonoscillatory whenever

$$
\int_{R}^{r} \sqrt{\chi(s)} \mathrm{d} s \int_{r}^{+\infty}\left(\frac{A(s)-\chi(s)}{\sqrt{\chi(s)}}\right) \mathrm{d} s \leq \frac{1}{4} \quad \text { for } r \text { big enough. }
$$

The last two inequalities are not particularly appealing, since they require a careful balancing of the integral behaviour of $\sqrt{\chi}$ on $[R, r]$ and on $[r,+\infty)$. In Chapter 5 , working directly on the ODE $\left(v z^{\prime}\right)^{\prime}+A v z=0$, we will prove a different, sharp oscillatory condition with a fairly neat expression in terms of the critical curve. Furthermore, our criterion will not require (4.21).

(2). First zeroes of solutions of (4.3) may appear even when $A(r)$ is sufficiently above $\chi(r)$ in a compact region, but small and below $\chi$ at infinity. For instance, consider the problem

$$
\left\{\begin{array}{l}
\left(r^{m-1} z^{\prime}(r)\right)^{\prime}+A(r) r^{m-1} z(r)=0 \quad \text { on } \mathbb{R}^{+}, \\
z(0)=z_{0}>0, \quad z^{\prime}(0)=0
\end{array}\right.
$$

with $m \geq 3,0 \leq A \in C^{0}\left(\mathbb{R}_{0}^{+}\right)$and

$$
A(r) \begin{cases}=z_{0}^{2}(m-2)^{2} r^{2(m-3)} & \text { on }\left[0,\left(\pi / z_{0}\right)^{\frac{1}{m-2}}\right] \\ \leq \frac{(m-2)^{2}}{4} \frac{1}{r^{2}} & \text { on }\left[\left(\pi / z_{0}\right)^{\frac{1}{m-2}}+1,+\infty\right) .\end{cases}
$$


Then, by Propositions 3.2, 3.5 and Remark 4.3, problem (4.26) has a unique $C^{2}$ solution $z$ on $\mathbb{R}_{0}^{+}$with finitely many zeroes, and it is immediate to verify that

$$
z(r) \equiv r^{2-m} \sin \left(z_{0} r^{m-2}\right) \quad \text { on }\left[0,\left(\pi / z_{0}\right)^{\frac{1}{m-2}}\right] .
$$

Thus, $z(r)$ has a first zero in $\left(\pi / z_{0}\right)^{1 /(m-2)}$. The following elementary computations serve the purpose to introduce what shall reveal to be a finite form condition for the existence of a first zero of $z$. We fix $0<R \leq r \leq\left(\pi / z_{0}\right)^{1 /(m-2)}$ and compute

$$
\int_{R}^{r}(\sqrt{A(s)}-\sqrt{\chi(s)}) \mathrm{d} s=z_{0}\left(r^{m-2}-R^{m-2}\right)-\frac{m-2}{2} \log \left(\frac{r}{R}\right) .
$$

Note that the LHS of the above equation measures the area (with sign) between the graph of $\sqrt{A(r)}$ and that of the critical curve $\sqrt{\chi(r)}$ on the interval $[R, r]$ before the first zero. A simple computation shows that

$$
-\frac{1}{2}\left(\log \int_{0}^{R} A(s) s^{m-1} \mathrm{~d} s+\log \int_{R}^{+\infty} s^{1-m} \mathrm{~d} s\right)=\log \left(\frac{\sqrt{3}}{z_{0}}\right)-(m-2) \log R .
$$

Thus, the difference between (4.28) and (4.29) is equal to

$$
\frac{(m-2)}{2} \log \left(\frac{R^{3}}{r}\right)+z_{0}\left(r^{m-2}-R^{m-2}\right)-\log \left(\frac{\sqrt{3}}{z_{0}}\right) .
$$

The above function on the region

$$
\mathcal{D}=\left\{(R, r) \in\left[0,\left(\pi / z_{0}\right)^{\frac{1}{m-2}}\right] \times\left[0,\left(\pi / z_{0}\right)^{\frac{1}{m-2}}\right]: r \geq R\right\}
$$

has a positive absolute maximum: indeed, it is positive when restricted to $R=$ $r \in\left[\left(\sqrt{3} / z_{0}\right)^{1 /(m-2)},\left(\pi / z_{0}\right)^{1 /(m-2)}\right]$. Concluding, by continuity for every choice of initial data $z_{0}$ we can find $0<R<r<\left(\pi / z_{0}\right)^{1 /(m-2)}$ such that

$$
\int_{R}^{r}(\sqrt{A(s)}-\sqrt{\chi(s)}) \mathrm{d} s>-\frac{1}{2}\left(\log \int_{0}^{R} A(s) s^{m-1} \mathrm{~d} s+\log \int_{R}^{+\infty} s^{1-m} \mathrm{~d} s\right) .
$$

We shall see below that the above inequality is the condition of Corollary 5.2 for the existence of a first zero. The interest on such a condition lies in the fact that only the LHS depends on $r$, thus (4.30) reveals how much $A$ must exceed $\chi$ on some compact region $[R, r]$ to force the existence of a first zero, and the bound is given only in terms of $A$ before $R$ and of $v(r)=r^{m-1}$.

\subsection{Stability, index of $-\Delta-q(x)$ and the uncertainty principle}

An easy but significant geometric application of Theorem 4.1 is the following spectral estimate for manifolds with a pole. For the convenience of the reader, we state part (i) under general assumptions on $M$, while for (ii) and (iii) we exploit our estimates.

Theorem 4.10. Let $(M,\langle\rangle$,$) be a complete m-dimensional Riemannian man-$ ifold with a pole $o \in M$. Denote with $r(x)$ the distance function from $o$. 
(i) Let $G \in C^{0}\left(\mathbb{R}_{0}^{+}\right)$and let $g \in C^{2}\left(\mathbb{R}_{0}^{+}\right)$be a solution of

$$
\left\{\begin{array}{l}
g^{\prime \prime}-G g \leq 0 \quad \text { on } \mathbb{R}_{0}^{+}, \\
g(0)=0, \quad g^{\prime}(0)=1 .
\end{array}\right.
$$

Assume that $g>0$ on $\mathbb{R}^{+}, g^{1-m} \in L^{1}(+\infty)$ and

$$
K_{\text {rad }}(x) \leq-G(r(x)) .
$$

Suppose that $q(x) \in L_{\mathrm{loc}}^{\infty}(M)$ satisfies

$$
q(x) \leq \chi_{g^{m-1}}(r(x)) \quad \text { on } M .
$$

Then, there exists a positive weak solution $w \in C^{2}(M \backslash\{o\}) \cap C^{1}(M)$ of

$$
\Delta w+q(x) w \leq 0
$$

such that

$$
w(x) \asymp-\sqrt{\int_{r(x)}^{+\infty} \frac{\mathrm{d} s}{g(s)^{m-1}}} \log \int_{r(x)}^{+\infty} \frac{\mathrm{d} s}{g(s)^{m-1}} \quad \text { as } \quad r(x) \rightarrow+\infty
$$

In particular,

$$
\lambda_{1}^{L}(M) \geq 0 \quad \text { with } L=-\Delta-q(x) .
$$

(ii) Assume

$$
m \geq 3, \quad K_{\mathrm{rad}}(x) \leq \frac{B^{2}}{(1+r(x))^{2}} \quad \text { on } M,
$$

for some $B \in[0,1 / 2]$, and that, outside some geodesic ball $B_{R}$,

$$
\begin{cases}q(x) \leq \frac{1}{4(1+r(x))^{2} \log ^{2}(1+r(x))} & \text { if } B=1 / 2, m=3 . \\ q(x) \leq \frac{\left(B^{\prime \prime}(m-1)-1\right)^{2}}{4 r(x)^{2}} & \text { if } B<\frac{1}{2} \text { or } B=\frac{1}{2}, m>3,\end{cases}
$$

where $B^{\prime \prime}=\frac{1}{2}\left(1+\sqrt{1-4 B^{2}}\right)$. Then, $L=-\Delta-q(x)$ has finite index.

(iii) Assume

$$
\begin{cases}\limsup _{r(x) \rightarrow+\infty}\left(q(x) r(x)^{-\alpha}\right)<\frac{B^{2}(m-1)^{2}}{4} & \text { if } \alpha>-2, \alpha \neq 0 ; \\ q(x) \leq \frac{B^{2}(m-1)^{2}}{4} \quad \text { outside some } B_{R}, & \text { if } \alpha=0 ; \\ q(x) \leq \frac{\left(B^{\prime}(m-1)-1\right)^{2}}{4(1+r(x))^{2}} \quad \text { outside some } B_{R}, & \text { if } \alpha=-2,\end{cases}
$$

where $B^{\prime}=\frac{1}{2}\left(1+\sqrt{1+4 B^{2}}\right)$. Then, $L=-\Delta-q(x)$ has finite index. 
Proof. (i) We let $A \in C^{0}\left(\mathbb{R}_{0}^{+}\right)$be such that $q(x) \leq A(r(x))$ on $M$ and, for some $r_{0}>0$,

$$
0 \leq A(r) \leq \chi_{g^{m-1}}(r) \quad \text { on } \mathbb{R}^{+}, \quad A(r) \equiv \chi_{g^{m-1}}(r) \quad \text { on }\left[r_{0},+\infty\right) .
$$

Let $z(r)$ be the $C^{2}$ solution of

$$
\left\{\begin{array}{l}
\left(g(r)^{m-1} z^{\prime}(r)\right)^{\prime}+A(r) g(r)^{m-1} z(r)=0 \quad \text { on } \mathbb{R}^{+} \\
z(0)=z_{0}>0, \quad z^{\prime}\left(0^{+}\right)=0,
\end{array}\right.
$$

which exists by Corollary 3.7. According to Theorem 4.1 and Proposition 4.7, by (4.40) $z$ is positive and satisfies

$$
z(r) \asymp-\sqrt{\int_{r}^{+\infty} \frac{\mathrm{d} s}{g(s)^{m-1}}} \log \int_{r}^{+\infty} \frac{\mathrm{d} s}{g(s)^{m-1}} \quad \text { as } r \rightarrow+\infty .
$$

Note that, by (4.41) and $A(r) \geq 0$ we deduce $z^{\prime}(r) \leq 0$. By the Laplacian comparison theorem and (4.32),

$$
\Delta r(x) \geq(m-1) \frac{g^{\prime}(r(x))}{g(r(x))} \quad \text { on } \quad M \backslash\{o\} .
$$

Having defined $w(x)=z(r(x)) \in C^{2}(M \backslash\{o\}) \cap C^{1}(M)$ we then have

$$
\begin{aligned}
\Delta w & =z^{\prime \prime}+z^{\prime} \Delta r \leq z^{\prime \prime}+(m-1) \frac{g^{\prime}}{g} z^{\prime} \\
& =g^{1-m}\left(g^{m-1} z^{\prime}\right)^{\prime}=-A(r) z \leq-q(x) w,
\end{aligned}
$$

pointwise on $M \backslash\{o\}$ and weakly on $M$, since $\Delta r$ has a mild singularity at $r=0$. The spectral estimate (4.35) follows from (4.33) and Theorem 1.33.

(ii) By Theorem 3.21 we can consider

$$
\begin{array}{ll}
g(r)=\sqrt{1+r} \log (1+r) & \text { when } B=1 / 2, m=3, \\
g(r)=r^{B^{\prime \prime}} & \text { when } B<1 / 2 \text { or } B=1 / 2, m>3 .
\end{array}
$$

Combining (3.56) and (3.57) with assumption (4.37), in both cases

$$
q(x) \leq \chi_{g^{m-1}}(r(x)) \quad \text { on } M \backslash B_{r_{1}},
$$

for every $r_{1} \geq R$. Choose $0 \leq A \in C^{0}\left(\left[r_{1},+\infty\right)\right)$ such that $q(x) \leq A(r(x))$ on $M \backslash B_{r_{1}}$ and (4.40) is met on $\left[r_{1},+\infty\right)$, and consider the problem

$$
\begin{cases}\left(g(r)^{m-1} z^{\prime}(r)\right)^{\prime}+A(r) g(r)^{m-1} z(r)=0 & \text { on }\left[r_{1},+\infty\right) \\ z\left(r_{1}\right)>0, & z^{\prime}\left(r_{1}\right)=0 .\end{cases}
$$

By Remark 4.3, the $C^{2}$ solution $z(r)$ of (4.44) is positive. Moreover, a first integration and the initial condition $z^{\prime}\left(r_{1}\right)=0$ give $z^{\prime} \leq 0$. This is essential for $w(x)=z(r(x))$ to be a weak solution of

$$
-L w=\Delta w+q(x) w \leq 0 \quad \text { on } M \backslash B_{r_{1}},
$$

as computation (4.43) shows. The finiteness of $\operatorname{ind}_{L}(M)$ is a consequence of Theorem 1.41. 
(iii) By the comparison Proposition 3.13, $\chi \geq \chi_{g^{m-1}}$ on $\mathbb{R}^{+}$, where $g$ solves (4.31) with equality sign and $G(r)=B^{2}\left(1+r^{2}\right)^{\alpha / 2}$. An application of Theorem 3.18 on the model manifold $\left(M_{g},\langle\rangle,\right)$, with metric $\langle\rangle=,\mathrm{d} r^{2}+g(r)^{2} \mathrm{~d} \theta^{2}$, gives

$$
\begin{array}{ll}
\chi_{g^{m-1}}(r) \gtrsim \frac{B^{2}(m-1)^{2}}{4} r^{\alpha} \quad \text { as } r \rightarrow+\infty, & \text { if } \alpha>-2 ; \\
\chi_{g^{m-1}}(r) \geq \frac{\left(B^{\prime}(m-1)^{2}-1\right)^{2}}{4(1+r)^{2}} & \text { if } \alpha=-2,
\end{array}
$$

from these and (4.39) we deduce, both for $\alpha>-2, \alpha \neq 0$ and $\alpha=-2, q(x) \leq$ $\chi_{f}(r(x))$ on $M \backslash B_{r_{1}}, r_{1}$ sufficiently large. The rest is again as in $(i i)$. When $\alpha=\overline{0}$, there is no need to require that $q(x)$ is strictly below $B^{2}(m-1)^{2} / 4$ near infinity, since by inequality (3.51) the less demanding requirement of (4.39) is enough.

REMARK 4.11. Item ( $i i)$ of the above theorem contains the case of Euclidean space, that is, $B=0$, and the required bound (4.37) on $q(x)$ becomes the well known

$$
q(x) \leq \frac{(m-2)^{2}}{4 r^{2}} \quad \text { outside some } B_{R}
$$

REMARK 4.12. With the aid of Proposition 3.23, item $(i i)$, we can weaken the assumption (4.38) by requiring $K_{\text {rad }} \leq-B^{2} r^{\alpha}$ outside some compact set, up to the further mild condition (3.65).

REMARK 4.13. To prove cases $($ ii) and (iii) we have, as a matter of fact, constructed a solution $w$ of $\Delta w+q(x) w \leq 0$ (outside some ball $B_{R}$ ) with the asymptotic behaviour (4.34) as $r \rightarrow+\infty$. As it is clear from Theorem 4.1 and Proposition 4.7 , if

$$
q(x) \leq k \chi_{g^{m-1}}(r(x)) \quad \text { on } \quad M \backslash B_{R},
$$

for some $k<1$ and $R>0$, the same procedure yields a solution $w$ satisfying

$$
w(x) \asymp\left[\int_{r(x)}^{+\infty} \frac{\mathrm{d} s}{g(s)^{m-1}}\right]^{(1-\sqrt{1-k}) / 2} \quad \text { as } r(x) \rightarrow+\infty .
$$

These explicit barriers will be useful later.

REMARK 4.14. It is worth to point out that, in the Euclidean setting, S. Agmon in [Agm85] has obtained sharp upper and lower bounds for the decay of eigenfunctions of $L=-\Delta-q(x)$ related to eigenvalues $\lambda<\inf \sigma_{\text {ess }}(L)$. His ODE approach, used to deal with the case $q(x)=o\left(r(x)^{-1}\right)$, has been recently extended by H. Kumura $[\mathbf{K u m}]$ in the setting of complete Riemannian manifolds. Their ODE arguments, however, are somewhat different from those described here. It would therefore be interesting to compare the two methods, or to achieve Agmon-Kumura results with the aid of the techniques developed in this paper. In this respect, we feel that next Sections 5.6 and 5.7 could be useful.

With a little change of perspective, Theorem 4.10 gives the following nonEuclidean extension of the uncertainty principle lemma in (2.41). We prove the theorem in two somehow different ways, which are closely related. The first one is by directly exploiting Theorem 4.10, and the second one relies on an important observation in [LW06]. 
THEOREM 4.15 (Non-Euclidean uncertainty principle). Let $(M,\langle\rangle$,$) be a com-$ plete Riemannian manifold of dimension $m \geq 2$ with a pole o and radial sectional curvature satisfying

$$
K_{\text {rad }}(x) \leq-G(r(x)),
$$

with $G \in C^{0}\left(\mathbb{R}_{0}^{+}\right)$. Let $g \in C^{2}\left(\mathbb{R}_{0}^{+}\right)$be a solution of

$$
\left\{\begin{array}{l}
g^{\prime \prime}-G g \leq 0 \quad \text { on } \mathbb{R}_{0}^{+}, \\
g(0)=0, \quad g^{\prime}(0)=1 .
\end{array}\right.
$$

Suppose that $g>0$ on $\mathbb{R}^{+}$and $g^{1-m} \in L^{1}(+\infty)$. Then, for every $u \in H^{1}(M)$,

$$
\int_{M}\left(\chi_{g^{m-1}} \circ r\right) u^{2} \leq \int_{M}|\nabla u|^{2} .
$$

First Proof. By the regularity of $g, \chi_{g^{m-1}} \in C^{0}\left(\left(0, \varepsilon_{0}\right]\right)$ for some $\varepsilon_{0}>0$. Choose $0<\varepsilon<\varepsilon_{0}$ and apply case $(i)$ of Theorem 4.10 with the choice

$$
q_{\varepsilon}(x)= \begin{cases}\inf _{[0, \varepsilon]} \chi_{g^{m-1}} & \text { if } r(x) \leq \varepsilon \\ \chi_{g^{m-1}}(r(x)) & \text { if } r(x) \geq \varepsilon\end{cases}
$$

to deduce $\lambda_{1}^{L_{\varepsilon}}(M) \geq 0$, where $L_{\varepsilon}=-\Delta-q_{\varepsilon}(x)$. Hence, for every $u \in C_{c}^{\infty}(M)$,

$$
\int_{M} q_{\varepsilon} u^{2} \leq \int_{M}|\nabla u|^{2} .
$$

Now observe that, if $M$ is complete, $H^{1}(M)$ is the closure of $C_{c}^{\infty}(M)$ in the $H^{1}$ norm. This can be seen as follows. For every $u \in H^{1}(M)$, consider a family of cut-off functions $\left\{\varphi_{r}\right\} \subset C_{c}^{\infty}(M)$ such that

$$
0 \leq \varphi_{r} \leq 1, \quad \varphi_{r} \equiv 1 \text { on } B_{r}, \quad \operatorname{supp}\left(\varphi_{r}\right) \subset B_{2 r}, \quad\left|\nabla \varphi_{r}\right| \leq \frac{C}{r},
$$

for some $C>0$ independent of $r$ (see [Gaf54]). It is straightforward to see that $u \varphi_{r} \rightarrow u$ in $H^{1}(M)$ as $r \rightarrow+\infty$. It is enough to approximate each $u \varphi_{r} \in H_{0}^{1}\left(B_{2 r}\right)$ by $C_{c}^{\infty}\left(B_{2 r}\right)$ functions $\left\{u_{r, j}\right\}_{j}$, and to use a Cantor diagonal argument. Therefore, (4.48) holds for every $u \in H^{1}(M)$. Since $0 \leq q_{\varepsilon} \uparrow \chi_{g^{m-1}}$ on $M$, letting $\varepsilon \rightarrow 0$ and using the monotone convergence theorem we reach the desired inequality.

SECOND PROOF. Consider the following function:

$$
\mathcal{G}(x)=\int_{r(x)}^{+\infty} \frac{\mathrm{d} s}{g^{m-1}(s)} .
$$

By the Laplacian comparison Theorem 1.17,

$$
\Delta \mathcal{G}=(m-1) \frac{g^{\prime}(r)}{g(r)^{m}}-\frac{1}{g(r)^{m-1}} \Delta r \leq 0
$$

on $M \backslash\{o\}$. Whence, for every $a \in \mathbb{R}^{+}$the function $\mathcal{G}_{a}=\min \{\mathcal{G}, a\}$ is positive, bounded on $M$ and it is a weak solution of $\Delta \mathcal{G}_{a} \leq 0$. A straightforward computation shows that $w=\sqrt{\mathcal{G}_{a}}$ is a positive, weak solution of

$$
\Delta w+\frac{\left|\nabla \log \mathcal{G}_{a}\right|^{2}}{4} w \leq 0 .
$$

By Theorem 1.33, for every $u \in \operatorname{Lip}_{c}(M)$

$$
\int \frac{\left|\nabla \log \mathcal{G}_{a}\right|^{2}}{4} u^{2} \leq \int|\nabla u|^{2}
$$


and letting $a \rightarrow+\infty$, by monotone convergence we get

$$
\int \frac{|\nabla \log \mathcal{G}|^{2}}{4} u^{2} \leq \int|\nabla u|^{2} \quad \forall u \in \operatorname{Lip}_{c}(M) .
$$

This can be extended to $u \in H^{1}(M)$ by density as in the previous proof. It is immediate to verify that

$$
\frac{|\nabla \log \mathcal{G}(x)|^{2}}{4}=\chi(r(x))
$$

Remark 4.16. Note that $\mathcal{G}$ in (4.49) is the transplantation to $M$ of the Green function $\overline{\mathcal{G}}(o, x)$ for the model $\left(M_{g}, \mathrm{~d} s^{2}\right)$, which exists by the non-parabolicity assumption $g^{1-m} \in L^{1}(+\infty)$ (see [Gri99]). The second proof of the above theorem is inspired by the following observation of $\mathrm{P}$. Li and J. Wang in [LW06]: consider a non-parabolic manifold $M$, possibly incomplete, and let $\mathcal{G}(x, y)$ be its minimal positive Green kernel. Then, for every fixed $y \in M$, the function

$$
\frac{\left|\nabla_{x} \log \mathcal{G}(x, y)\right|^{2}}{4}=\frac{\left|\nabla_{x} \mathcal{G}\right|^{2}}{4 \mathcal{G}^{2}}(x, y)
$$

is a good Hardy weight for the uncertainty principle lemma, that is,

$$
\int \frac{\left|\nabla_{x} \mathcal{G}\right|^{2}}{4 \mathcal{G}^{2}} u^{2} \leq \int|\nabla u|^{2} \quad \forall u \in \operatorname{Lip}_{c}(M) .
$$

Indeed, having observed that $\mathcal{G}_{a}(x)=\min \{\mathcal{G}(x, y), a\}$ is superharmonic for every $a \in \mathbb{R}^{+}$, the proof of (4.51) goes along the same lines as the second proof of Theorem 4.15 .

It should be observed that, in the very recent paper $[\mathbf{A K}], \mathrm{K}$. Akutagawa and H. Kumura have proved a similar uncertainty principle lemma. More precisely, let $M$ be a complete manifold with a pole. Then, for every $u \in C_{c}^{\infty}(M)$,

$$
\int_{M}|\nabla u|^{2} \geq \int_{M}\left[\frac{1}{4 r^{2}}+\frac{1}{4}(\Delta r)^{2}-\frac{1}{2}|\operatorname{Hess} r|^{2}-\frac{1}{2} \operatorname{Ricc}(\nabla r, \nabla r)\right] u^{2} .
$$

The idea of the proof is to combine the one-dimensional Hardy inequality (see for instance [HLP52], Theorem 327), an integration by parts in normal coordinates and formula (1.46). Since, in (4.52), $\Delta r$ and $\mid$ Hess $\left.r\right|^{2}$ appear with different signs, it is difficult to estimate the RHS by means of comparison results. It would be interesting to compare (4.52) and (4.47) for a general manifold with a pole. However, we postpone this matter to a forthcoming paper. A somehow related question will be discussed after the next estimates for $\lambda_{1}^{L}\left(B_{R}\right), \lambda_{1}^{L}(M)$ and $\inf \sigma_{\text {ess }}(L)$. In the case $L=-\Delta$, the result below should be compared with Theorem 2.17, item (ii). The interested reader can also consult the papers by M.A. Pinsky [Pin78] (for surfaces with a pole), R. Brooks [Bro81] and H. Donnelly [Don81b].

Proposition 4.17. Let $(M,\langle\rangle$,$) be a complete Riemannian manifold with a$ pole $o$, and let $K_{\mathrm{rad}}, G, g$ satisfy the assumptions of Theorem 4.15. Let $L=-\Delta-$ $q(x)$, where $q(x) \in L_{\mathrm{loc}}^{\infty}(M)$ Then,

$$
\begin{gathered}
\lambda_{1}^{L}\left(B_{R}\right) \geq \inf _{x \in B_{R}}\left(\chi_{g^{m-1}}(r(x))-q(x)\right), \quad \lambda_{1}^{L}(M) \geq \inf _{x \in M}\left(\chi_{g^{m-1}}(r(x))-q(x)\right) ; \\
\inf \sigma_{\mathrm{ess}}(L) \geq \liminf _{r(x) \rightarrow+\infty}\left(\chi_{g^{m-1}}(r(x))-q(x)\right) .
\end{gathered}
$$


In particular, if $\left(\chi_{g^{m-1}}(r(x))-q(x)\right) \rightarrow+\infty$, then L has only discrete spectrum.

Proof. These inequalities follow immediately from Rayleigh characterization, the decomposition Theorem 1.38 and the uncertainty principle. Indeed, for the last relation,

$$
\begin{aligned}
\inf \sigma_{\mathrm{ess}}(L) & =\lim _{r \rightarrow+\infty}\left(\inf _{0 \neq \phi \in C_{c}^{\infty}\left(M \backslash B_{r}\right)} \frac{\int_{M}|\nabla \phi|^{2}-q \phi^{2}}{\int_{M} \phi^{2}}\right) \\
& \geq \lim _{r \rightarrow+\infty}\left(\inf _{0 \neq \phi \in C_{c}^{\infty}\left(M \backslash B_{r}\right)} \frac{\int_{M}\left(\chi_{g^{m-1}}-q\right) \phi^{2}}{\int_{M} \phi^{2}}\right) \\
& \geq \lim _{r \rightarrow+\infty} \inf _{M \backslash B_{r}}\left(\chi_{g^{m-1}}-q\right) .
\end{aligned}
$$

The other estimates are proved similarly. If $\chi_{g^{m-1}}(r(x))-q(x) \rightarrow+\infty$ as $x$ diverges, then $\sigma_{\text {ess }}(L)=\emptyset$, and by the min-max theorem $\sigma(L)$ is a divergent sequence of nonnegative eigenvalues, each of finite multiplicity.

REMARK 4.18. As an easy consequence of our estimates for $\chi_{g^{m-1}}(r)$, in particular inequality (3.51), we recover a theorem of McKean [McK70]. Indeed, if $K_{\mathrm{rad}} \leq-B^{2}$ on $M$, the next lower bound for the spectral radius of $-\Delta$ on $M$ holds:

$$
\lambda_{1}^{-\Delta}(M) \geq \inf _{r \in \mathbb{R}^{+}} \chi_{g^{m-1}}(r) \geq \frac{B^{2}(m-1)^{2}}{4} .
$$

Remark 4.19. Suppose that $L=-\Delta$. Then, combining Corollary 3.24 and Proposition 4.17, we immediately get a proof of item (ii) of Corollary 2.17 by using the critical curve instead of comparisons for $\Delta r$.

On the links between $\chi$ and $\tilde{\chi}$, I. We pause for a moment to comment on the estimates in (4.53). Assume for simplicity that $q(x) \equiv 0$, that is, that $L=-\Delta$. It is useful to compare the proof of Proposition 4.17 with the classical method to prove lower bounds of $\lambda_{1}^{-\Delta}\left(B_{R}\right)$ that we described in Proposition 2.14. As we realize by comparing (4.53) and (2.26), we need a closer look to the mutual relationship between the $C^{1}$ curves

$$
\chi_{g^{m-1}}(r) \quad \text { and } \quad\left(\frac{m-1}{2} \frac{g^{\prime}(r)}{g(r)}\right)^{2}=\tilde{\chi}_{g^{m-1}}(r),
$$

since $\chi$ and $\tilde{\chi}$ enter in spectral estimates with identical tasks. Note that $\tilde{\chi}$ is the modified critical function of (3.35) for $f(r)=g(r)^{m-1}$. For convenience, we omit writing the subscript $f$. The above problem is nontrivial, and we begin with some observations that will be recalled in next sections to deal with part of the question. First, comparing with (3.29) we observe that $\chi$ and $\tilde{\chi}$ have a different behaviour near $r=0$, since by the properties of $g(r)$

$$
\widetilde{\chi}(r) \sim \frac{(m-1)^{2}}{4 r^{2}} \quad \text { as } r \rightarrow 0^{+} .
$$

In [BR97] the authors found, for Euclidean and hyperbolic spaces, the first instance of a critical curve, that for the present considerations we shall call $\Theta(r)$. They proved that, if $A$ lies below $\Theta$, a solution $z$ of $\left(g^{m-1} z^{\prime}\right)^{\prime}+A g^{m-1} z=0$ is positive 
and has an explicit lower bound at infinity. Although the lower bounds coincide with those in (4.1), for the hyperbolic case they found for $\Theta$ the curve

$$
\frac{B^{2}(m-1)^{2}}{4} \operatorname{coth}^{2}(B r)=\tilde{\chi}(r) \quad(\text { they excluded, however, the case } m=2) .
$$

One might ask if this is a general property, that is, if $\tilde{\chi}$ can replace $\chi$ as a critical curve for (at least $C^{1}$ ) volume functions. If this were true, $-\Delta-q$ must have non-negative spectral radius for every $q \leq \tilde{\chi}$. By the approximation procedure of Theorem 4.15, this is equivalent to requiring that the uncertainty principle holds with $\chi$ replaced by $\tilde{\chi}$. By (4.54), this is impossible if $m=2$. Indeed, if $u=1$ in a ball $B_{1}$ around $o$, from $g(s) \sim s, g^{\prime}(s) \rightarrow 1$ as $s \rightarrow 0$ we deduce that, for some small $C>0$,

$$
\int_{B_{1}} \widetilde{\chi}_{g^{m-1}}(r(x)) \mathrm{d} V(x) \geq C \int_{0}^{1}\left(\frac{g^{\prime}(s)}{g(s)}\right)^{2} g(s) \mathrm{d} s=+\infty .
$$

Therefore, if $m=2, \widetilde{\chi}$ can never be used as a critical curve. This justifies why, in [BR97], the authors assume $m \geq 3$ even for the hyperbolic case. The situation for $m \geq 3$ is more subtle. However, it is known that on $\mathbb{R}^{m}$ the constant $(m-2)^{2} / 4$ is sharp for the uncertainty principle. Since, on $\mathbb{R}^{m}, \widetilde{\chi}(r)=(m-1)^{2} /\left(4 r^{2}\right), \widetilde{\chi}$ is not a critical curve for $\mathbb{R}^{m}$ for any $m$. Essentially, the problem is that $\tilde{\chi}$ is too big with respect to $\chi$ in a neighbourhood of $+\infty$. Indeed,

$$
\frac{\tilde{\chi}(r)}{\chi(r)} \rightarrow\left(\frac{m-1}{m-2}\right)^{2}>1 \quad \text { as } r \rightarrow+\infty .
$$

However, by (3.34), for non-polynomial volume growths $f(r)$ as in (3.33) it holds $\tilde{\chi} \sim \chi$ as $r \rightarrow+\infty$, so we need finer estimates. This discussion will be considered in detail in the remark "On the links between $\chi$ and $\tilde{\chi}$, III", at the end of Section 5.3 below. The key difference between $\chi$ and $\tilde{\chi}$ is that $\chi(r)$ takes into account the values of $f$ on the whole $[r,+\infty)$, while $\tilde{\chi}$ merely depends on $f$ in arbitrarily small neighbourhoods of $r$. For this reason, since $\lambda_{1}^{-\Delta}\left(B_{R}\right)$ only depends on the geometry of $B_{R}, \widetilde{\chi}$ should be, at least conceptually, more suitable than $\chi$ to yield a lower bound for $\lambda_{1}^{-\Delta}\left(B_{R}\right)$. Indeed, at least for small $R$, by comparing (3.29) and (4.54) the curve $\tilde{\chi}$ yields better estimates for $\lambda_{1}^{-\Delta}\left(B_{R}\right)$ than $\chi$. However, deciding which curve gives better estimates for $\lambda_{1}^{-\Delta}\left(B_{R}\right)$ when $R$ is big seems more complicated. In this respect, the following ODE characterization of $\chi$ in terms of $\tilde{\chi}$ is useful. Suppose that $f$ is non-decreasing on $\mathbb{R}^{+}$. Then, from their very definitions,

$$
2 \sqrt{\chi}-2 \sqrt{\tilde{\chi}}=\frac{\mathrm{d}}{\mathrm{d} r}\left(-\log \int_{r}^{+\infty} \frac{\mathrm{d} s}{f(s)}-\log f(s)\right)=\frac{\mathrm{d}}{\mathrm{d} r} \log (2 \sqrt{\chi}),
$$

hence $y(r)=2 \sqrt{\chi(r)} \in C^{1}\left(\mathbb{R}^{+}\right)$is a solution of Bernoulli equation

$$
y^{\prime}=y^{2}-2 y \sqrt{\tilde{\chi}} .
$$

From the form of the ODE, we argue that

$$
\tilde{\chi}(r)>\chi(r) \quad(\text { resp. }<\chi(r)) \quad \text { if and only if } \quad \chi^{\prime}(r)<0 \quad(\text { resp. }>0),
$$

and that $\chi \equiv \tilde{\chi}$ if and only if both are constants, which implies $f(r)=\Lambda \exp \{a r\}$ for some $\Lambda, a>0$. 


\subsection{A comparison at infinity for nonlinear PDE}

The spectral estimates of Theorem 4.10 also provide barriers at infinity helpful to compare subsolutions and supersolutions of semilinear elliptic equations on unbounded domains. This is the core of the following theorem, which improves on Theorem 3.1 and Corollary 3.3 of [RRV97]. In what follows we consider the prototype nonlinearity $b(x) u^{\sigma}, \sigma>1$ of Yamabe-type equations. Note that the case of a bounded domain has already been dealt with in Proposition 2.32. The basic step is the following general

TheOrem 4.20. Let $(M,\langle\rangle$,$) be a Riemannian manifold, let q(x) \in L_{\mathrm{loc}}^{\infty}(M)$ and let $L=-\Delta-q(x)$. Assume that there exists a nonzero, weak solution $0 \leq$ $w \in C^{0}(M \backslash \bar{\Omega}) \cap H_{\mathrm{loc}}^{1}(M \backslash \bar{\Omega})$ of the inequality $L w \geq 0$ on $M \backslash \bar{\Omega}$, for some relatively compact domain $\Omega$. Let

$$
0 \leq b(x) \in L_{\mathrm{loc}}^{\infty}(M), \quad \sigma>1,
$$

and suppose that $u, v \in \operatorname{Lip}_{\text {loc }}(M)$ are weak solutions on $M$ of the following inequalities:

$$
\begin{cases}\Delta u+q(x) u \leq b(x) u^{\sigma} & u>0 \quad \text { on } M \\ \Delta v+q(x) v \geq b(x) v^{\sigma} & v \geq 0 \quad \text { on } M\end{cases}
$$

If

$$
u-v=o(w) \quad \text { as } x \text { diverges, }
$$

then one of the following cases occur:

(1) $v \leq u$ on $M$;

(2) $b(x)=0$ a.e. on $M, v=C u$ for some constant $C>1$ and both satisfy (4.58) with equality signs.

Proof. By the maximum principle ([GT98], p.35), $w>0$. First, we extend $w$ to a positive function $\widetilde{w}$ on the whole $M$. For instance, this can be done by taking a relative compact set $\Omega^{\prime}$ such that $\Omega \Subset \Omega^{\prime}$, a cut-off function $0 \leq \psi \leq 1$ compactly supported in $\Omega^{\prime}$ and satisfying $\psi \equiv 1$ on $\Omega$, and defining $\widetilde{w}=\psi+w(1-\psi)$. Note that $\widetilde{w}=w$ on $M \backslash \overline{\Omega^{\prime}}$, so that $L \widetilde{w} \geq 0$ weakly on $M \backslash \overline{\Omega^{\prime}}$. For notational convenience, we write again $w$ and $\Omega$ in place of $\widetilde{w}$ and $\Omega^{\prime}$. Let $\varepsilon>0$, and define $u_{\varepsilon}=u+\varepsilon w$ on $M$. Then, $u_{\varepsilon}$ is a weak solution of $\Delta u_{\varepsilon}+q u_{\varepsilon} \leq b(x) u^{\sigma}-\varepsilon L w$, that is, by definition and by (4.58), the following inequalities hold for every $0 \leq \phi \in \operatorname{Lip}_{c}(M)$ :

$$
\begin{aligned}
\text { (i) }-\int_{M}\left\langle\nabla u_{\varepsilon}, \nabla \phi\right\rangle+\int_{M} q u_{\varepsilon} \phi & \leq \int_{M} b(x) u^{\sigma} \phi-\varepsilon \int_{M} w L \phi \\
\text { (ii) }-\int_{M}\langle\nabla v, \nabla \phi\rangle+\int_{M} q v \phi & \geq \int_{M} b(x) v^{\sigma} \phi .
\end{aligned}
$$

Suppose that case (1) does not occur. Then, by (4.59) the Lipschitz function $\gamma_{\varepsilon}=\left(v^{2}-u_{\varepsilon}^{2}\right)_{+}$is compactly supported and nonzero for $\varepsilon$ sufficiently small, hence

$$
\Theta_{\varepsilon}=\left\{x \in M: v(x)>u_{\varepsilon}(x)\right\}
$$

is a nonempty, relatively compact open set. Since $v>u_{\varepsilon} \geq \varepsilon \inf _{\Theta_{\varepsilon}} w \geq C(\varepsilon)>0$ on $\Theta_{\varepsilon}$, for some positive constant $C(\varepsilon)>0$, the functions $\phi_{1}=\gamma_{\varepsilon} / u_{\varepsilon}, \phi_{2}=\gamma_{\varepsilon} / v$ 
are admissible for (4.60). Choosing $\phi_{1}$ in $(i)$ and $\phi_{2}$ in $(i i)$, and subtracting the two resulting inequalities we deduce

$$
\begin{aligned}
& -\int_{\Theta_{\varepsilon}}\left\langle\frac{\nabla u_{\varepsilon}}{u_{\varepsilon}}-\frac{\nabla v}{v}, \nabla \gamma_{\varepsilon}\right\rangle+\int_{\Theta_{\varepsilon}}\left(\frac{\left|\nabla u_{\varepsilon}\right|^{2}}{u_{\varepsilon}^{2}}-\frac{|\nabla v|^{2}}{v^{2}}\right) \gamma_{\varepsilon} \\
& \leq \int_{\Theta_{\varepsilon}} b(x)\left(u^{\sigma} / u_{\varepsilon}-v^{\sigma-1}\right) \gamma_{\varepsilon}-\varepsilon \int_{M} w L\left(\gamma_{\varepsilon} / u_{\varepsilon}\right)
\end{aligned}
$$

Inserting the expression for $\gamma_{\varepsilon}$ and rearranging we get

$$
\begin{aligned}
& \int_{\Theta_{\varepsilon}}\left|\nabla u_{\varepsilon}-\frac{u_{\varepsilon}}{v} \nabla v\right|^{2}+\int_{\Theta_{\varepsilon}}\left|\nabla v-\frac{v}{u_{\varepsilon}} \nabla u_{\varepsilon}\right|^{2} \\
& \leq \int_{\Theta_{\varepsilon}} b(x)\left(u^{\sigma} / u_{\varepsilon}-v^{\sigma-1}\right) \gamma_{\varepsilon}-\varepsilon \int_{M} w L\left(\gamma_{\varepsilon} / u_{\varepsilon}\right) .
\end{aligned}
$$

Let $V$ be a smooth, relatively compact domain such that $\Omega \Subset V$, and let $0 \leq \psi \leq 1$ be a smooth function such that $\psi=1$ on $\Omega$ and $\psi \equiv 0$ on $M \backslash \bar{V}$. Then, from the properties of $w$

$$
\int_{M} w L\left(\gamma_{\varepsilon} / u_{\varepsilon}\right)=\int_{M} w L\left(\psi \gamma_{\varepsilon} / u_{\varepsilon}\right)+\int_{M} w L\left((1-\psi) \gamma_{\varepsilon} / u_{\varepsilon}\right) \geq \int_{M} w L\left(\psi \gamma_{\varepsilon} / u_{\varepsilon}\right) .
$$

Since $u$ is bounded from below by a positive constant on $\bar{V}$, applying the dominated convergence theorem we deduce that

$$
\lim _{\varepsilon \rightarrow 0} \varepsilon\left|\int_{M} w L\left(\psi \gamma_{\varepsilon} / u_{\varepsilon}\right)\right| \leq \lim _{\varepsilon \rightarrow 0} \varepsilon\left[\int_{V}|\nabla w|\left|\nabla\left(\psi \gamma_{\varepsilon} / u_{\varepsilon}\right)\right|+\left|q w \psi \gamma_{\varepsilon} / u_{\varepsilon}\right|\right]=0 .
$$

Hence, letting $\varepsilon \rightarrow 0$ in (4.61), using Fatou lemma and the last two inequalities we finally get

$$
0 \leq \int_{\{v>u\}}\left|\nabla u-\frac{u}{v} \nabla v\right|^{2} \leq \int_{\{v>u\}} b(x)\left(u^{\sigma-1}-v^{\sigma-1}\right)\left(v^{2}-u^{2}\right) \leq 0 .
$$

Therefore, $v / u$ is constant on every connected component $\Gamma$ of $\{v>u\}$. Clearly, $\Gamma$ must have no boundary, for otherwise letting $x \rightarrow \partial \Gamma$ we would deduce $u=v$ on $\Gamma$, contradiction. By connectedness, $v=C u$ on $M$ for some $C>1$ and inserting into (4.62) we deduce

$$
\int_{M} b(x)\left(1-C^{2}\right)\left(1-C^{\sigma-1}\right) u^{\sigma+1} \equiv 0 .
$$

Case (2) follows immediately.

REMARK 4.21. We recall that, by Theorem 1.41, the existence of $w$ satisfying the assumptions of the above theorem is equivalent to the requirement $\operatorname{ind}_{L}(M)<$ $+\infty$.

REMARK 4.22. As in Theorem 3.1 of [RRV97], $\operatorname{ind}_{L}(M)<+\infty$ can be replaced with the existence of a solution $w$ of

$$
L w \geq-\sigma b(x) u^{\sigma-1} w \quad \text { weakly on } M \backslash \bar{\Omega} .
$$

In other words, $\operatorname{ind}_{L}(M)<+\infty$ can be substituted for the requirement that the solution $u$ of the Yamabe equation is a stable solution (in the sense of calculus of variations) outside some compact set. 
Clearly, the above comparison has an obvious, companion uniqueness result for weak solutions of $\Delta u+q(x) u=b(x) u^{\sigma}$, where $b \geq 0$ and $b \not \equiv 0$ on $M$. Note that, by the maximum principle, each non-negative solution $u$ of $\Delta u+q(x) u \leq b(x) u^{\sigma}$ is either strictly positive or identically zero. If the assumption $\operatorname{ind}_{L}(M)<+\infty$ is strengthened to $\lambda_{1}^{L}(M) \geq 0$, with minor modifications in the proof one can even consider the case $u \equiv 0$ (set $u_{\varepsilon}=\varepsilon w$ ). The resulting statement is a Liouville type theorem that we present for the particular case of manifolds with a pole. Suppose therefore that $(M,\langle\rangle$,$) has a pole o$ and radial sectional curvature controlled as usual:

$$
K_{\text {rad }}(x) \leq-G(r(x)) \quad \text { on } M,
$$

for some $G \in C^{0}\left(\mathbb{R}_{0}^{+}\right)$. Once a solution $g$ of

$$
\left\{\begin{array}{l}
g^{\prime \prime}-G g \leq 0 \quad \text { on } \mathbb{R}^{+}, \\
g(0)=0, \quad g^{\prime}(0)=1
\end{array}\right.
$$

such that $g>0$ on $\mathbb{R}^{+}$and $g^{1-m} \in L^{1}(+\infty)$ is given, by Theorem 4.10 condition

$$
q(x) \leq \chi_{g^{m-1}}(r(x)) \quad \text { on } M \backslash B_{R}, \quad \text { for some } R>0
$$

$\operatorname{implies}_{\operatorname{ind}}(M)<+\infty$, and the same with $R=0$ ensures $\lambda_{1}^{L}(M) \geq 0$. Furthermore, we can construct a radial weak solution $w$ of $L w \geq 0$ with the asymptotic

$$
w(x) \asymp-\sqrt{\int_{r(x)}^{+\infty} \frac{\mathrm{d} s}{g(s)^{m-1}}} \log \int_{r(x)}^{+\infty} \frac{\mathrm{d} s}{g(s)^{m-1}} \quad \text { as } r(x) \rightarrow+\infty .
$$

As an immediate application of Theorem 4.20 and the above discussion, we state the following

Corollary 4.23. Let $(M,\langle\rangle$,$) be a manifold with a pole o$ and radial sectional curvature satisfying (4.63). Let $g$ be a solution of (4.64) such that $g>0$ on $\mathbb{R}^{+}$ and $g^{1-m} \in L^{1}(+\infty)$. Let $q(x) \in L_{\text {loc }}^{\infty}(M)$, and assume that

$$
q(x) \leq \chi_{g^{m-1}}(r(x)) \quad \text { on } M \backslash\{o\} .
$$

Let $\sigma>1$ and choose $0 \leq b(x) \in L_{\mathrm{loc}}^{\infty}(M), b \neq \equiv 0$ on $M$. Suppose that $0 \leq v \in$ $\operatorname{Lip}_{\text {loc }}(M)$ satisfies

$$
\Delta v+q(x) v \geq b(x) v^{\sigma}
$$

and

$$
v(x)=o\left(-\sqrt{\int_{r(x)}^{+\infty} \frac{\mathrm{d} s}{g(s)^{m-1}}} \log \int_{r(x)}^{+\infty} \frac{\mathrm{d} s}{g(s)^{m-1}}\right) \quad \text { as } r(x) \rightarrow+\infty .
$$

Then, $v \equiv 0$ on $M$.

REMARK 4.24. It is worth to realize that, if $g$ satisfies (4.64) with the equality sign, one does not obtain a sharper result. This is due to the appearance of two opposite effects. Indeed, consider the solution $h$ of

$$
\left\{\begin{array}{l}
h^{\prime \prime}-G h=0, \\
h(0)=0, \quad h^{\prime}(0)=1 .
\end{array}\right.
$$

Sturm argument and Proposition 3.12 imply $g \leq h$ and $\chi_{g^{m-1}} \leq \chi_{h^{m-1}}$, so that (4.65) is more demanding than the requirement $q(x) \leq \chi_{h^{m-1}}(r(x))$. On the contrary, since $-\sqrt{x} \log x$ is increasing near 0 , substituting $g$ with $h$ in (4.68) gives a 
smaller bound at infinity. Thus, the above result has to be interpreted as follows: if $q(x)$ is sufficiently small to lie below $\chi_{g^{m-1}}(r(x))$, and not only below $\chi_{h^{m-1}}(r(x)$ ), then for $v \equiv 0$ to hold on $M$ it is enough that (4.68) is met with $g$ instead of the larger $h$.

We spend a few words to comment on the role of the spectral radius of $L$, and to compare Theorem 4.20 and Corollary 4.23 with the previous literature. Suppose for convenience that $b(x)>0$ on $M$. As we have seen in the proof of Theorem 2.30 , if $\lambda_{1}^{L}(M)<0$ there is no obstacle to the existence of a nonzero solution $0 \leq v \in \operatorname{Lip}_{\text {loc }}(M)$ of

$$
\Delta v+q(x) v \geq b(x) v^{\sigma} .
$$

Indeed, $v$ can even be compactly supported. By the subsolution-supersolution method and the positivity of $b(x)$, this is enough to construct positive solutions $u$ of $\Delta u+q(x) u=b(x) u^{\sigma}$. On the contrary, if $\lambda_{1}^{L}(M) \geq 0$ each positive solution $w$ of $L w \geq 0$ is a barrier that forces a minimal growth of any subsolution $v \geq 0$. Such $w$ has been specified by imposing an upper bound on the radial sectional curvature of $M$. The same idea is the core of other type of Liouville theorems, although they are obtained with quite different techniques. For example, by Theorem 1.3 and Section 3 of [BRS98a] no positive, bounded subsolution $v$ can exists if $\lambda_{1}^{L}(M) \geq 0$ and $v$ satisfy some suitable integrability conditions. These can be rephrased in terms of upper bounds of $v$ once a controlled decay is imposed on $q(x), b(x)$ and $\operatorname{Ricc}(\nabla r, \nabla r)$ is bounded from below. It is curious to observe that the geometrical requirement is opposite to (4.63). We will now show that these results do not contain Theorem 4.20 .

Towards this aim, let $\left(M_{g}, \mathrm{~d} s^{2}\right)$ be a model manifold with metric, in polar coordinates, $\mathrm{d} s^{2}=\mathrm{d} r^{2}+g(r)^{2} \mathrm{~d} \theta^{2}$, where $g \in C^{\infty}\left(\mathbb{R}_{0}^{+}\right), g>0$ on $\mathbb{R}^{+}$and

$$
g(r)= \begin{cases}r & \text { if } r \in[0,1] ; \\ \exp \left\{\frac{1}{m-1} r^{\alpha} \log ^{\beta} r\right\} & \text { if } r \in[2,+\infty), \quad \alpha>0, \beta \geq 0 .\end{cases}
$$

Clearly, setting $G=-g^{\prime \prime} / g=-K_{\text {rad }}, g$ solves (4.64). The volume element is $g^{m-1}$, and choosing a $L_{\text {loc }}^{\infty}$ function $q(x)$ such that $q(x)=\chi_{g^{m-1}}(r(x))$ on $M \backslash B_{1}$, the supersolution $w$ has the behaviour

$$
\begin{aligned}
w(r) & \asymp-\sqrt{\int_{r}^{+\infty} \frac{\mathrm{d} s}{g(s)^{m-1}}} \log \int_{r}^{+\infty} \frac{\mathrm{d} s}{g(s)^{m-1}} \\
& \asymp r^{\frac{\alpha+1}{2}} \log ^{\frac{\beta}{2}} r \exp \left\{-\frac{m-1}{2} r^{\alpha} \log ^{\beta} r\right\}
\end{aligned}
$$

as $r \rightarrow+\infty$. Hence, assuming $u-v=o(w)$, by (4.66) there exists $C>0$ such that, for $r>>1$,

$$
\frac{1}{\int_{\partial B_{r}}(u-v)^{2}} \geq \frac{C}{\int_{\partial B_{r}} w^{2}} \asymp \frac{1}{r^{\alpha+1} \log ^{\beta} r} \in L^{1}(+\infty) \text { since } \alpha>0 .
$$

In other words, (4.68) in general does not imply

$$
\left(\int_{\partial B_{r}}(u-v)^{2}\right)^{-1} \notin L^{1}(+\infty),
$$


thus Theorem 4.20 is not contained in Theorem 4.1 of [BRS98a]. Note that the exponent 2 in (4.73) is special for the validity of the uniqueness result. Indeed, it cannot be substituted with any $p>2$, see [BRS98a] and [BRS98b]. The same model manifold can be used to prove that Corollary 4.23 is not contained in Theorem 1.3 of [BRS98a] (see also Theorem 8.9 of [PRS08]). This last result states that a bounded, non-negative solution $v \in C^{2}(M)$ of (4.67) is identically zero provided

$$
\begin{array}{ll}
\text { (1) } \quad \lambda_{1}^{L}(M) \geq 0, & \text { (2) } \quad b(x)>0, q(x) \leq C b(x) \text { for some } C>0, \\
\text { (3) } q(x) v^{2} \in L^{1}(M), \quad \text { (4) } \quad\left(\int_{\partial B_{r}} v^{2}\right)^{-1} \notin L^{1}(+\infty) .
\end{array}
$$

Indeed, choose $0<q=b \leq \chi$ on $M, q=b=\chi$ on $M \backslash B_{2}$, so that (1), (2) are met. By Corollary $4.23, v \equiv 0$ provided $v=o(w)$, where $w$ has the asymptotic behaviour in (4.71). By (4.72), the condition $v=o(w)$ does not automatically imply (4). As for (3), by the expression of $\chi$, for every $r \geq 2$

$$
\int_{\partial B_{r}} q w^{2} \asymp\left[g(r)^{m-1} \int_{r}^{+\infty} \frac{\mathrm{d} s}{g(s)^{m-1}}\right]^{-1} \log ^{2} \int_{r}^{+\infty} \frac{\mathrm{d} s}{g(s)^{m-1}} \asymp r^{3 \alpha-1} \log ^{3 \beta} r
$$

which is non-integrable at infinity. Hence, by the coarea formula, not even (3) is a consequence of $v=o(w)$.

Once we specialize Theorem 4.20 to manifolds with a pole and to the explicit $g$ of Theorem 4.10 (items (ii) and (iii)), we obtain the next result that improves on Theorem $C$ of [BR97].

Corollary 4.25. Let $(M,\langle\rangle$,$) be a complete Riemannian manifold of dimen-$ sion $m$ with a pole $o$, and let $q(x), b(x) \in L_{\text {loc }}^{\infty}(M)$. Suppose that one of the set of assumptions (ii), (iii) of Theorem 4.10 is met, and that $b(x) \geq 0, b \not \equiv 0$. Let $\sigma>1$, and let $u, v \in \operatorname{Lip}_{\text {loc }}(M)$ be such that

$$
\left\{\begin{array}{lll}
\Delta u+q(x) u \leq b(x) u^{\sigma}, & & u>0 \text { on } M \\
\Delta v+q(x) v \geq b(x) v^{\sigma}, & v \geq 0 & \text { on } M .
\end{array}\right.
$$

Then, $v \leq u$ on $M$ provided

$$
\begin{array}{ll}
u-v=o\left(r^{\left.-\frac{(m-1) B^{\prime \prime}-1}{2} \log r\right)}\right. & \text { for }(i i),\left\{\begin{array}{l}
B \in[0,1 / 2) \text { or } \\
B=1 / 2, m>3 ;
\end{array}\right. \\
u-v=o\left(\log ^{-\frac{1}{2}} r \log \log r\right) & \text { for }(i i), B=1 / 2, m=3 ; \\
u-v=o\left(r^{1+(m+1) \frac{\alpha}{8}} \exp \left\{-\frac{B(m-1)}{2+\alpha} r^{1+\frac{\alpha}{2}}\right\}\right) & \text { for } \quad(i i i), \alpha \geq 0 ; \\
u-v=o\left(r^{1+\frac{\alpha}{4}} \exp \left\{-\frac{B(m-1)}{2+\alpha} r^{1+\frac{\alpha}{2}}\right\}\right) & \text { for } \quad(i i i), \alpha \in(-2,0) ; \\
u-v=o\left(r^{-\frac{(m-1) B^{\prime}-1}{2}} \log r\right) & \text { for }(i i i), \alpha=-2 .
\end{array}
$$

as $r \rightarrow+\infty$, where $B^{\prime \prime}=\frac{1}{2}\left(1+\sqrt{1-4 B^{2}}\right)$ and $B^{\prime}=\frac{1}{2}\left(1+\sqrt{1+4 B^{2}}\right)$.

Proof. By Theorem 4.10, if $q(x)$ satisfy the requirements of cases $(i i)$ and (iii) then $\operatorname{ind}_{L}(M)<+\infty$, where $L=-\Delta-q(x)$. Furthermore, by Remark 4.13, 
there exists a positive solution $w$ of $L w \geq 0$, outside some compact set, with the behaviour (4.66) as $r(x) \rightarrow+\infty$. Since $b \not \equiv 0$ excludes case (2) of Theorem 4.20 , to prove that $v \leq u$ on $M$ it is enough to check, for each explicit $g(r)$ as in the proof of Theorem 4.10, that the asymptotic (4.66) for $w(x)$ coincides with the bound in (4.75).

As for $(i i)$,

$$
G(r)=-\frac{B^{2}}{1+r^{2}}, \quad 0 \leq B \leq \frac{1}{2},
$$

and a good choice is to consider

$$
\begin{array}{ll}
g(r)=\sqrt{1+r} \log (1+r) & \text { when } B=1 / 2, m=3 ; \\
g(r)=r^{B^{\prime \prime}} & \text { when } B<1 / 2 \text { or } B=1 / 2, m>3 .
\end{array}
$$

Estimate (3.54) gives (4.75) at infinity. To deal with case (iii), set

$$
G(r)=B^{2}\left(1+r^{2}\right)^{\alpha / 2} \text {. }
$$

When $\alpha \geq 0$, we can choose

$$
g(r)=r^{1 / 2} I_{\frac{1}{2+\alpha}}\left(\frac{2 B}{2+\alpha} r^{1+\frac{\alpha}{2}}\right),
$$

up to a positive normalizing constant (see also the proof of Theorem 3.18). Estimate (4.75) follows from (3.43), (3.44). When $\alpha \in(-2,0), g(r)$ has the form

$$
g(r)=\frac{1}{B} \sinh \left(\frac{2 B}{2+\alpha}\left[(1+r)^{1+\frac{\alpha}{2}}-1\right]\right),
$$

and (4.75) is a consequence of (3.40). In the polynomial case $\alpha=-2$ we use

$$
g(r)=(1+r)^{B^{\prime}} .
$$

Note that the different conditions at 0 with respect to those of (4.31) are, however, compatible with Sturm argument. Indeed, if $h$ solves (4.31) with equality sign, $\left(h^{\prime} g-h g^{\prime}\right)^{\prime} \geq 0$ and $\left(h^{\prime} g-h g^{\prime}\right)(0)=1$, hence $h^{\prime} / h \geq g^{\prime} / g$ on $\mathbb{R}^{+}$. By the comparison Proposition 3.12, (4.76) is suitable for (4.44) and to yield the radial supersolution $w(x)$.

REMARK 4.26. Observe that, in (4.75), the estimate for case $($ iii), $\alpha \in(-2,0)$ fits with that for (iii), $\alpha \geq 0$ as $\alpha \uparrow 0$. Analogously, that for $(i i i), \alpha=-2$ approaches the bound in $(i i)$ when $B \rightarrow 0$. As a particular case, we recover the asymptotic behaviours in Theorem $C$ of $[\mathbf{B R 9 7}]$ for $\mathbb{R}^{m}$ and for the hyperbolic space $\mathbb{H}_{B}^{m}$ :

$$
\begin{array}{ll}
u-v=o\left(r^{-\frac{m-2}{2}} \log r\right) & \text { for } \mathbb{R}^{m}, m \geq 3 ; \\
u-v=o\left(r \exp \left\{-\frac{B(m-1)}{2} r\right\}\right) & \text { for } \mathbb{H}_{B}^{m}, m \geq 2, B>0 .
\end{array}
$$

REMARK 4.27. According to Remark 4.13, if we replace assumptions $($ ii), $($ iii) of Theorem 4.10 with the corresponding requirements on $q(x)$ that imply

$$
q(x) \leq k \chi(r(x)) \quad \text { on } M \backslash \bar{B}_{R},
$$

for some $k<1$, we can provide a whole range of bounds of type (4.75) depending on $k$. We leave the computational details to the interested reader. 
The next Corollary applies the above comparison result to a relative of the Yamabe problem.

Corollary 4.28. Let $(M,\langle\rangle$,$) be a complete Riemannian manifold of di-$ mension $m \geq 3$ with a pole $o$ and scalar curvature $s(x) \leq 0, s \not \equiv 0$. Define $q(x)=-s(x) / c_{m}$, where $c_{m}=4(m-1) /(m-2)$. Suppose that one of the set of assumptions (ii), $($ iii $)$ of Theorem 4.10 is met. Let $f: M \rightarrow M$ be a conformal diffeomorphism that preserves the scalar curvature, and define $u>0$ according to $f^{*}\langle\rangle=,u^{\frac{4}{m-2}}\langle$,$\rangle . If the decay conditions in (4.75) are met with v \equiv 1$, then $f$ is an isometry.

ProOF. In our assumptions, by (2.55) $u>0$ is a solution of

$$
0=\Delta u-\frac{s(x)}{c_{m}} u+\frac{s(x)}{c_{m}} u^{\frac{m+2}{m-2}}=\Delta u+q(x) u-q(x) u^{\frac{m+2}{m-2}} .
$$

Since $v \equiv 1$ is clearly another solution, by Corollary 4.25 we deduce $u \leq 1$. Reversing the role of $u$ and $v$ we deduce $u \geq 1$, thus $u \equiv 1$ and $f$ is an isometry.

Our next task is a brief discussion on the sharpness of Corollary 4.25. Towards this aim, we consider $M=\mathbb{R}^{m}, m \geq 3$, and the coefficients $q(x), b(x)$ satisfying

$$
q(x) \leq \frac{(m-2)^{2}}{4 r(x)^{2}}, \quad b(x) \leq \frac{r(x)^{(m-2)(\sigma-1) / 2}}{(\log r(x))^{\sigma+1}(\log \log r(x))(\log \log \log r(x))^{2}},
$$

and equal to the upper bounds for $r(x)>>1$. Then, it has been proved in [BRS98b] that $\Delta u+q(x) u=b(x) u^{\sigma}$ has a family of distinct, positive solutions $u_{a}$, $a>0$, satisfying

$$
u_{a}(o)=a, \quad u_{a}(x) \sim r(x)^{-\frac{m-2}{2}} \log r(x) \quad \text { as } r(x) \rightarrow+\infty,
$$

coherently with case $(i i), B=0$ of (4.75). As a second example, we recall that in Section 4 of [BRS98a]. Consider the standard hyperbolic space $\mathbb{H}^{m}=\mathbb{H}_{1}^{m}$. By means of suitable conformal transformations, we are going to produce a family of solutions $\left\{u_{a}\right\}$ of

$$
\Delta u_{a}-\frac{s(x)}{c_{m}} u_{a}=u_{a}^{\frac{m+2}{m-2}}, \quad \text { where } \frac{s(x)}{c_{m}}=-\frac{m(m-1)(m-2)}{4(m-1)}=-\frac{m(m-2)}{4} .
$$

Towards this aim, let $D^{m}$ be the unit disk of $\mathbb{R}^{m}$, and let $\langle\rangle,, \widetilde{\langle,\rangle}$ be, respectively, the Euclidean and the Poincarè metric on $D^{m}$ :

$$
\widetilde{\langle,\rangle}=\frac{4}{\left(1-|x|^{2}\right)^{2}}\langle,\rangle=u^{\frac{4}{m-2}}\langle,\rangle, \quad \text { where } \quad u=\left(\frac{2}{1-|x|^{2}}\right)^{\frac{m-2}{2}} .
$$

Let $a>0$, and consider the solutions

$$
\beta_{a}(r)=\frac{\left(a^{2}-r^{2}\right)^{-\frac{m-2}{2}}}{m(m-2) a^{2}} \quad \text { of } \quad\left\{\begin{array}{l}
\beta_{a}^{\prime \prime}+\frac{m-1}{r} \beta_{a}^{\prime}=\beta_{a}^{\frac{m+2}{m-2}} \text { on }(0,1) \\
\beta_{a}(0)=\frac{1}{m(m-2) a^{2}}, \quad \beta_{a}^{\prime}(0)=0 .
\end{array}\right.
$$

Clearly, they give rise to a family of solutions

$$
w_{a}(x)=\beta_{a}(r(x)) \quad \text { of } \quad \Delta_{\mathbb{R}^{m}} w_{a}=w_{a}^{\frac{m+2}{m-2}} \quad \text { on } \quad\left(D^{m},\langle,\rangle\right) .
$$

By (2.56), the functions $v_{a}=u^{-1} w_{a}$ are solutions of

$$
\widetilde{\Delta} v_{a}+\frac{m(m-2)}{4} v_{a}=u^{-\frac{m+2}{m-2}} w_{a}^{\frac{m+2}{m-2}}=v_{a}^{\frac{m+2}{m-2}} \quad \text { on }\left(D^{m}, \widetilde{\langle,\rangle}\right) .
$$


Now, consider the radial model $\left(M_{g}, \mathrm{~d} s^{2}\right)$ of the hyperbolic space, with metric, in polar coordinates, $\mathrm{d} s^{2}=\mathrm{d} r^{2}+\sinh ^{2} r \mathrm{~d} \theta^{2}$. The map $T:\left(M_{g}, \mathrm{~d} s^{2}\right) \rightarrow\left(D^{m}, \widetilde{\langle,\rangle}\right)$ given, in polar coordinates, by

$$
T \quad: \quad(r, \theta) \longmapsto\left(\tanh \frac{r}{2}, \theta\right)
$$

is an isometry between the two models of $\mathbb{H}^{m}$, so that

$$
u_{a}(x)=v_{a}(T(x))=\left(2 \cosh ^{2} \frac{r(x)}{2}\right)^{-\frac{m-2}{2}} \beta_{a}\left(\tanh \frac{r(x)}{2}\right)
$$

is a family of distinct solutions of

$$
\Delta u_{a}+\frac{m(m-2)}{4} u_{a}=u_{a}^{\frac{m+2}{m-2}}
$$

with the property that

$$
u_{a}(x) \sim\left[\frac{2^{-(m-2) / 2}}{m(m-2) a^{2}}\left(a^{2}-1\right)^{-(m-2) / 2}\right] e^{-\frac{m-2}{2} r(x)} \quad \text { as } r(x) \rightarrow+\infty .
$$

This decay is slower than the desired $r \exp \{-(m-1) r / 2\}$. The reason is that the potential $q(x)=m(m-2) / 4$ is much below the critical curve $\chi$ of $\mathbb{H}^{m}$; indeed, by

$\chi(r(x)) \geq \frac{(m-1)^{2}}{4}=\frac{(m-1)^{2}}{m(m-2)}\left(-\frac{s(x)}{c_{m}}\right)=\frac{1}{k} q(x) \quad$ where $k=\frac{m(m-2)}{(m-1)^{2}}<1$.

Consequently, the bounds (4.75) can be improved, according to Remarks 4.13 and 4.27 , to the following requirement for $\mathbb{H}^{m}$ :

$u-v=o\left(\left[\int_{r}^{+\infty} \frac{\mathrm{d} s}{\sinh ^{m-1} s}\right]^{(1-\sqrt{1-k}) / 2}\right)=o\left(e^{-\frac{m-1}{2}(1-\sqrt{1-k}) r}\right)=o\left(e^{-\frac{m-2}{2} r}\right)$,

so $e^{-(m-2) r / 2}$ is sharp as the minimal growth allowed when $L$ is the conformal Laplacian on $\mathbb{H}^{m}$. As far as we know, Corollary 4.25 is not contained in previous results. In this respect, note also that it does not overlap with the very general comparison Theorem 17 of [PRS06].

\subsection{Yamabe type equations with a sign-changing nonlinearity}

In Section 4.3 we have considered, on a non-compact manifold $M$, Yamabe-type equations

$$
\Delta u+q(x) u=b(x) u^{\sigma}
$$

where the coefficient $b(x)$ of the nonlinearity is non-negative. The case where $b(x)$ in (4.78) is allowed to change sign reveals to be the most challenging one, and very little is known either about the existence or about the non-existence of positive solutions. As an application of the techniques presented in this paper, we are going to obtain a sharp existence result on model manifolds under suitable growth conditions on $|b(x)|$. What we present in this section is part of the forecoming paper [BMR].

Historically, to the best of our knowledge, the existence problem for (4.78) with $\sigma \leq(m+2) /(m-2)$ has been mainly studied via a combination of concentrationcompactness methods and variants of the mountain pass theorem inspired by the seminal paper of H. Brezis and L. Nirenberg, [BN83]. Among the literature we 
limit ourselves to quote [Zha04] for both a sharp result and an up-to-date account of the problem, and we suggest the reader to consult the references therein for futher insight. Usually, this variational approach requires the validity of some global Sobolev-type inequality on $M$ (commonly expressed as the positivity of a Yamabe-type invariant) which, on non-compact manifolds, turns out to be a quite restrictive assumption. In the Euclidean setting and when $a(x)=0$, W.M. Ni [Ni82], and later W.Y. Ding and W.M. Ni [DN85], have studied the problem with different techniques and have given optimal conditions for the existence of positive solutions of (4.78) in terms of the growth of $|b(x)|$. Furthemore, they have described a whole variety of phenomena to illustrate how subtle is the dependence of $u$ upon the behaviour of $b(x)$. As an application of the results in Chapter 4, we are able to extend the original ideas in [Ni82] to the case of model manifolds and general $a(x)$. These results seem to be new even for the hyperbolic space, see [BR97]. First, we recall our starting point, $\mathrm{Ni}$ result, which will be recovered as a corollary of the main Theorem 4.35 .

Theorem 4.29 ([Ni82], Theorem 1.4). Consider the Euclidean space $\mathbb{R}^{m}, m \geq$ 3 , and let $\widetilde{s}(x) \in C^{\infty}\left(\mathbb{R}^{m}\right)$ be a function satisfying

$$
|\widetilde{s}(x)| \leq \frac{C}{r(x)^{l}},
$$

for some $l>2$ and for some constant $C>0$. Then, the Euclidean metric $\langle$,$\rangle can$ be conformally deformed to a complete smooth metric $\widetilde{\langle,\rangle}$ of scalar curvature $\widetilde{s}(x)$ and satisfying

$$
C_{1}\langle,\rangle_{x} \leq \widetilde{\langle,\rangle_{x}} \leq C_{2}\langle,\rangle_{x} \quad \forall x \in \mathbb{R}^{m},
$$

for some $0<C_{1} \leq C_{2}$. Furthermore, $C_{2}$ can be chosen to be as small as we wish.

Note that the bound in (4.79) can be improved. Indeed, already in [Ni82] it is shown that

$$
|\widetilde{s}(x)| \leq \frac{C}{r(x)^{2} \log ^{2} r(x)} \quad \text { for } r(x)>>1 .
$$

is sufficient, and later on M. Naito, [Nai84], has refined (4.81) up to the following sufficient condition for the existence of the conformal deformation:

$$
|\widetilde{s}(x)| \leq B(r(x)), \quad \text { for some } B \text { such that } r B(r) \in L^{1}(+\infty) .
$$

Moreover, he has also proved that (4.82) is sufficient to improve (4.80) to the property that

$$
\widetilde{\langle,\rangle_{x}} \rightarrow C\langle,\rangle_{x} \quad \text { as } r(x) \rightarrow+\infty
$$

for some constant $C \in\left(0, C_{2}\right]$. The case $C=0$, that is, when the conformal factor $u \rightarrow 0$ as $r(x) \rightarrow+\infty$, reveals some further problem, and the sole (4.82) is not sufficient to ensure the existence of a positive $u$ decaying to 0 , as has been shown in $[\mathbf{L N 8 8}]$.

Condition (4.82) is essentially sharp. In fact, by Theorem 1.13' of [Ni82] or Theorem A of [BR97], no conformal deformations of $\mathbb{R}^{m}$ exist whenever the new scalar curvature $\widetilde{s}(x)$ is required to satisfy

$$
\widetilde{s}(x) \leq-\frac{C}{r(x)^{2} \log r(x)} \quad \text { for } r>>1 .
$$

for some constant $C>0$. 
REMARK 4.30. In virtue of $\mathrm{Ni}$ and Naito results, one could ask whether the condition

$$
\widetilde{s}(x) \leq-B(r(x)) \leq 0 \quad \text { and } \quad r B(r) \notin L^{1}(+\infty)
$$

prevents from the existence of any positive solution of $\Delta u=-\widetilde{s}(x) u^{\sigma}$ on $\mathbb{R}^{m}$. This is conjectured in [CL87] and, to the best of our knowledge, it is still an open problem. In [Lin85], [KN84], [CL87], a number of steps have been moved towards the solution of this conjecture, giving rise to non-existence conditions slightly more demanding than (4.84), see in particular Theorems 2.2 and 2.3 in [CL87].

We now come to our main results. Let $\left(M_{g}, \mathrm{~d} s^{2}\right)$ be a model manifold, and denote with $v(r)=\omega_{m-1} g(r)^{m-1}$ the volume growth of its geodesic spheres centered at the reference origin. Note that $v$ satisfies (V1), (V2), (V3). We further assume $\left(\mathrm{V}_{\mathrm{L} 1}\right)$. We need the following

Lemma 4.31. Suppose that $v$ is as above, and let $A \in L_{\text {loc }}^{\infty}\left(\mathbb{R}_{0}^{+}\right)$be such that $A<k \chi$ on $\mathbb{R}^{+}$, for some $k \in(-\infty, 1]$. Let $B \in L_{\mathrm{loc}}^{\infty}\left(\mathbb{R}_{0}^{+}\right), B \geq 0$, and $\sigma>1$. For each fixed $\alpha>0$, consider a Lip loc solution $z_{\alpha}$ of

$$
\left\{\begin{array}{l}
\left(v z_{\alpha}^{\prime}\right)^{\prime}+A v z_{\alpha}+B v\left|z_{\alpha}\right|^{\sigma-1} z_{\alpha}=0 \quad \text { on }\left[0, \varepsilon_{\alpha}\right) \\
z_{\alpha}(0)=\alpha>0, \quad z_{\alpha}^{\prime}(0)=0 .
\end{array}\right.
$$

for some $\varepsilon_{\alpha}>0$. Let $h$ and $\bar{h}$ be positive solutions of

$$
\left\{\begin{array}{l}
\left(v h^{\prime}\right)^{\prime}+A v h \geq 0 \quad \text { on } \mathbb{R}^{+} \\
h(0)=1, \quad h^{\prime}(0)=0 .
\end{array}, \quad\left\{\begin{array}{l}
\left(v \bar{h}^{\prime}\right)^{\prime}+A v \bar{h} \leq 0 \quad \text { on } \mathbb{R}^{+} \\
\bar{h}(0)=1, \quad \bar{h}^{\prime}(0)=0 .
\end{array}\right.\right.
$$

on $\mathbb{R}_{0}^{+}$. Suppose that $B(r)$ satisfies

$$
\begin{array}{ll}
B(r) \leq-C \frac{\chi(r)-A(r)}{h(r)^{\sigma}} \sqrt{\int_{r}^{+\infty} \frac{\mathrm{d} s}{v(s)}} \log \int_{r}^{+\infty} \frac{\mathrm{d} s}{v(s)} & \text { if } k=1 ; \\
B(r) \leq C \frac{k \chi(r)-A(r)}{h(r)^{\sigma}}\left[\int_{r}^{+\infty} \frac{\mathrm{d} s}{v(s)}\right]^{(1-\sqrt{1-k}) / 2} & \text { if } k \in(-\infty, 1),
\end{array}
$$

when $r \geq r_{1}$, for some large $r_{1}$ and for some constant $C>0$. Then, there exists $\alpha_{0}>0$ such that, for every $\alpha \in\left(0, \alpha_{0}\right), z_{\alpha}$ can be extended to a positive, locally Lipschitz solution on $\mathbb{R}^{+}$of (4.85) satisfying

$$
\frac{\alpha}{2} \bar{h}(r) \leq z_{\alpha}(r) \leq \alpha h(r) \quad \text { on } \mathbb{R}^{+} .
$$

REMARK 4.32. The local existence for (4.85) is ensured, for instance, via the Picard iteration argument or a modification of Proposition 4.3. This last procedure also gives $z_{\alpha}^{\prime}(0)=0$, and positivity follows from the initial data and from continuity. Furthermore, in our assumptions, Theorem 4.1 guarantees the existence of positive solutions $h, \bar{h}$ of $(4.86)$.

Proof of Lemma 4.31. On the interval $\left[0, \varepsilon_{\alpha}\right)$ we consider the function $\xi=$ $z_{\alpha} / h$. From (4.85), (4.86) and $B \geq 0$ we deduce

$$
\left\{\begin{array}{l}
\left(h^{2} v \xi^{\prime}\right)^{\prime} \leq 0 \quad \text { on }\left(0, \varepsilon_{\alpha}\right) \\
\xi(0)=\alpha, \quad \xi^{\prime}(0)=0
\end{array}\right.
$$


Integrating we obtain $\xi^{\prime} \leq 0$, and therefore $\xi(r) \leq \xi(0)=\alpha$. In other words,

$$
z_{\alpha}(r) \leq \alpha h(r) \quad \text { on }\left[0, \varepsilon_{\alpha}\right),
$$

We now look for a lower bound of $z_{\alpha}$ on $\left[0, \varepsilon_{\alpha}\right)$. Towards this aim we observe that, since $A(r)<k \chi(r)$ on $\mathbb{R}_{0}^{+}$, we can define $\widetilde{A} \in \operatorname{Lip}_{\text {loc }}\left(\mathbb{R}_{0}^{+}\right)$in such a way that

$$
A<\widetilde{A} \leq k \chi \quad \text { on } \mathbb{R}^{+}, \quad \widetilde{A} \equiv k \chi \quad \text { on }\left[r_{1},+\infty\right),
$$

Next, let $w$ be the solution of

$$
\left\{\begin{array}{l}
\left(v w^{\prime}\right)^{\prime}+\widetilde{A} v w=0 \quad \text { on } \mathbb{R}^{+} \\
w(0)=1, \quad w^{\prime}(0)=0 .
\end{array}\right.
$$

Then, by Theorem $4.1 w>0$ on $\mathbb{R}^{+}$and it satisfies the estimate

$$
\begin{array}{ll}
w(r) \asymp-\sqrt{\int_{r}^{+\infty} \frac{\mathrm{d} s}{v(s)}} \log \int_{r}^{+\infty} \frac{\mathrm{d} s}{v(s)} & \text { if } k=1 ; \\
w(r) \asymp\left[\int_{r}^{+\infty} \frac{\mathrm{d} s}{v(s)}\right]^{(1-\sqrt{1-k}) / 2} & \text { if } k \in(-\infty, 1) .
\end{array}
$$

Hence, using assumption (4.87) we deduce the existence of a constant $C_{1}>0$ sufficiently large such that

$$
B(r) \leq C_{1} \frac{[\widetilde{A}(r)-A(r)] w(r)}{h(r)^{\sigma}} \quad \text { on } \mathbb{R}^{+} .
$$

Note that, for the existence of $C_{1}$, it is necessary that $A<\widetilde{A}$ on $\mathbb{R}^{+}$and therefore that the strict inequality $A<k \chi$ holds.

For any $\beta>0$ we set $w_{\beta}=\beta w$, and consider $\varphi=z_{\alpha}-w_{\alpha / 2}$. Then, by the initial conditions for $z_{\alpha}$ and $w_{\alpha / 2}, \varphi>0$ on some maximal interval $\left[0, \bar{\varepsilon}_{\alpha}\right) \subset\left[0, \varepsilon_{\alpha}\right)$. From (4.85), (4.90), (4.92) and (4.89) we get

$$
\begin{aligned}
\left(v \varphi^{\prime}\right)^{\prime} & =-B v\left|z_{\alpha}\right|^{\sigma}-A v z_{\alpha}+\widetilde{A} v w_{\alpha / 2} \\
& =-A v \varphi+(\widetilde{A}-A) v w_{\alpha / 2}-B v\left|z_{\alpha}\right|^{\sigma} \\
& \geq-A v \varphi+(\widetilde{A}-A) v w_{\alpha / 2}-C_{1} v \frac{(\widetilde{A}-A) w}{h^{\sigma}}(\alpha h)^{\sigma} \\
& =-A v \varphi+(\widetilde{A}-A) v w_{\alpha / 2}\left(1-2 C_{1} \alpha^{\sigma-1}\right),
\end{aligned}
$$

hence $\left(v \varphi^{\prime}\right)^{\prime}+A v \varphi \geq 0$ on $\left[0, \bar{\varepsilon}_{\alpha}\right)$ provided $\alpha \leq\left(2 C_{1}\right)^{-1 /(\sigma-1)}=\alpha_{0}$. Next, on $\left[0, \bar{\varepsilon}_{\alpha}\right)$ we define $\eta=\varphi / \bar{h}$. Using (4.86) and $\varphi>0$ we get

$$
\begin{cases}\left(\bar{h}^{2} v \eta^{\prime}\right)^{\prime} \geq 0 & \text { on }\left(0, \bar{\varepsilon}_{\alpha}\right) \\ \eta(0)=\alpha / 2, & \eta^{\prime}(0)=0 .\end{cases}
$$

Integrating, we get $\eta^{\prime} \geq 0$, hence from $\eta(r) \geq \eta(0)=\alpha / 2$ we finally get $\eta \geq \alpha / 2$. Since, by construction, $\eta\left(\bar{\varepsilon}_{\alpha}\right)=0$ whenever $\bar{\varepsilon}_{\alpha}<\varepsilon_{\alpha}$, we deduce that necessarily $\bar{\varepsilon}_{\alpha}=\varepsilon_{\alpha}$ and thus

$$
z_{\alpha} \geq w_{\alpha / 2}+\frac{\alpha}{2} \bar{h} \quad \text { on }\left[0, \varepsilon_{\alpha}\right),
$$


completing the proof of (4.88) restricted to $\left[0, \varepsilon_{\alpha}\right)$. Since $h, \bar{h}, w$ are defined and positive on $\mathbb{R}^{+}, z_{\alpha}$ cannot explode in a finite time and can therefore be extended to a positive solution on the whole $\mathbb{R}^{+}$.

REMARK 4.33. Without loss of generality, we can assume that $h=\bar{h}$ is the solution of $\left(v h^{\prime}\right)^{\prime}+A v h=0$ with initial condition $h(0)=1, h^{\prime}(0)=0$. Indeed, if we call $y(r)$ such a solution, by Sturm argument $\bar{h} \leq y \leq h$ on $\mathbb{R}^{+}$. We have preferred to keep $h, \bar{h}$ distinct since, to make condition (4.87) more explicit, we only need an explicit $h$ that solves a differential inequality.

REMARK 4.34. We observe that the first step in the above proof consists of getting rid of the linear term $A v z$ by taking a positive solution $h$ of $\left(v h^{\prime}\right)^{\prime}+A v h=0$ and considering $\xi=z_{\alpha} / h$. The price we have to pay is that a weight $h^{2}$ for the volume is introduced. However, as soon as we have a good control on $h$, this trick enables us to extend many classical results. For a detailed discussion, we refer the reader to the next Section 5.6.

THEOREM 4.35. Let $\left(M_{g}, \mathrm{~d} s^{2}\right)$ be an m-dimensional model manifold, denote with $v=\omega_{m-1} g^{m-1}$ the volume growth of geodesic spheres centered at the reference origin, and suppose that $\left(\mathrm{V}_{\mathrm{L} 1}\right)$ is met. Let $\sigma>1$, and let $A(r(x)) \in C_{\mathrm{loc}}^{2, \mu}\left(M_{g}\right)$, $0<\mu \leq 1$ be a radial function satisfying

$$
A(r)<k \chi(r) \quad \text { on } \mathbb{R}^{+},
$$

for some $k \in(-\infty, 1]$. Let $h, \bar{h}$ be positive, $C^{2}$ solutions of $(4.86)$ on $\mathbb{R}^{+}$. Consider a function $b(x) \in C_{\mathrm{loc}}^{2, \mu}\left(M_{g}\right)$ satisfying

$$
\begin{aligned}
|b(x)| & \leq-C \frac{\chi(r(x))-A(r(x))}{h(r(x))^{\sigma}} \sqrt{\int_{r(x)}^{+\infty} \frac{\mathrm{d} s}{v(s)}} \log \int_{r(x)}^{+\infty} \frac{\mathrm{d} s}{v(s)} \quad \text { if } k=1 ; \\
|b(x)| \leq C \frac{k \chi(r(x))-A(r(x))}{h(r(x))^{\sigma}}\left[\int_{r(x)}^{+\infty} \frac{\mathrm{d} s}{v(s)}\right]^{(1-\sqrt{1-k}) / 2} & \text { if } k \in(-\infty, 1),
\end{aligned}
$$

outside some ball, and for some $C>0$. Then, the equation

$$
\Delta u+A(r(x)) u-b(x) u^{\sigma}=0
$$

possesses infinitely many $C_{\mathrm{loc}}^{2, \mu}$ solutions on $M_{g},\left\{u_{j}\right\}_{j \in \mathbb{N}}$. For each of them, there exist constants $0<C_{1, j} \leq C_{2, j}$ such that

$$
C_{1, j} \bar{h}(r(x)) \leq u_{j}(x) \leq C_{2, j} h(r(x)) \quad \text { on } M_{g} .
$$

Furthermore, $C_{2, j} \downarrow 0$ as $j \rightarrow+\infty$. If $A(r)$ and $b \in C^{\infty}\left(M_{g}\right)$, then $\left\{u_{j}\right\} \subset$ $C^{\infty}\left(M_{g}\right)$.

Proof. First of all we prove the theorem in case $h=\bar{h}=y$ is a solution of

$$
\left\{\begin{array}{l}
\left(v y^{\prime}\right)^{\prime}+A v y=0 \\
y(0)=1 \quad y^{\prime}(0)=0 .
\end{array}\right.
$$

Next we choose a function $B(r) \geq 0$ on $\mathbb{R}_{0}^{+}, B \in L_{\text {loc }}^{\infty}\left(\mathbb{R}_{0}^{+}\right)$such that $|b(x)| \leq$ $B(r(x))$ on $M_{g}$ and satisfying (4.87). This is possible because of (4.95). By Lemma 
4.31, there exists $\alpha_{0}>0$ such that for each $\alpha \in\left(0, \alpha_{0}\right)$ we have a positive solution $z_{\alpha}$ on $\mathbb{R}_{0}^{+}$of $(4.85)$. Setting $\omega_{\alpha}(x)=z_{\alpha}(r(x)), \omega_{\alpha}$ solves

$$
\begin{aligned}
\Delta \omega_{\alpha}+A \omega_{\alpha}-b \omega_{\alpha}^{\sigma} & \leq \Delta \omega_{\alpha}+A \omega_{\alpha}+B \omega_{\alpha}^{\sigma} \\
& =z_{\alpha}^{\prime \prime}+(m-1) \frac{g^{\prime}}{g} z_{\alpha}^{\prime}+A z_{\alpha}+B z_{\alpha}^{\sigma} \\
& =v^{-1}\left[\left(v z_{\alpha}^{\prime}\right)^{\prime}+A v z_{\alpha}+B v z_{\alpha}^{\sigma}\right]=0
\end{aligned}
$$

and has the property that

$$
\frac{\alpha}{2} y(r(x)) \leq \omega_{\alpha}(x) \leq \alpha y(r(x))
$$

on $M_{g}$. Next, we define

$$
y_{\alpha}=\frac{\alpha}{2} y-z_{\alpha / 4}
$$

Then, $y_{\alpha}(0)=\alpha / 4$ and, by $(4.88)$,

$$
\frac{\alpha}{2} y \geq y_{\alpha}=\frac{\alpha}{2} y-z_{\alpha / 4} \geq\left(\frac{\alpha}{2}-\frac{\alpha}{4}\right) y=\frac{\alpha}{4} y .
$$

Therefore, using (4.99),

$$
\frac{\alpha}{4} y \leq y_{\alpha} \leq \frac{\alpha}{2} y \leq \omega_{\alpha} \leq \alpha y
$$

Furthermore,

$$
\left(v y_{\alpha}^{\prime}\right)^{\prime}+A v y_{\alpha}=B v z_{\alpha / 4}^{\sigma}=B v\left(\frac{z_{\alpha / 4}}{\frac{\alpha}{2} h-z_{\alpha / 4}}\right)^{\sigma} y_{\alpha}^{\sigma} .
$$

But

and it follows that $y_{\alpha}$ solves

$$
\frac{z_{\alpha / 4}}{\frac{\alpha}{2} h-z_{\alpha / 4}} \geq \frac{\frac{\alpha}{8} h}{\frac{\alpha}{2} h}=\frac{1}{4},
$$

$$
\left(v y_{\alpha}^{\prime}\right)^{\prime}+A v y_{\alpha} \geq B v 4^{-\sigma} y_{\alpha}^{\sigma}
$$

Defining $\bar{y}_{\alpha}=4^{-\frac{\sigma}{\sigma-1}} y_{\alpha}$ we have

$$
\left(v \bar{y}_{\alpha}^{\prime}\right)^{\prime}+A v \bar{y}_{\alpha} \geq B v \bar{y}_{\alpha}^{\sigma} .
$$

As a consequence, $\bar{\omega}_{\alpha}(x)=\bar{y}_{\alpha}(r(x))$ satisfies

$$
\Delta \bar{\omega}_{\alpha}+A \bar{\omega}_{\alpha}-b \bar{\omega}_{\alpha}^{\sigma} \geq(B-b) \bar{\omega}_{\alpha}^{\sigma} \geq 0
$$

and

$$
4^{\frac{-\sigma}{\sigma-1}} \frac{\alpha}{4} y(r(x)) \leq \bar{\omega}_{\alpha}(x) \leq 4^{\frac{-\sigma}{\sigma-1}} \frac{\alpha}{2} y(r(x)) \leq \frac{\alpha}{2} y(r(x)) \leq \omega_{\alpha}(x)
$$

on $M_{g}$. By the monotone iteration scheme, [Sat72], there exists a solution $u_{\alpha}(x)$ of (4.96) satisfying

$$
\bar{\omega}_{\alpha} \leq u_{\alpha} \leq \omega_{\alpha}
$$

on $M_{g}$. Furthermore, if $A(r)$ and $b$ are smooth, then $u_{\alpha}$ is smooth by elliptic regularity. From (4.101) and (4.102) it follows immediately that

$$
4^{-\frac{2 \sigma+1}{\sigma-1}} \alpha y(r(x)) \leq u_{\alpha}(x) \leq \alpha y(r(x))
$$

The procedure can now be iterated, simply replacing $\alpha=\alpha_{1}$ with, say, $\alpha_{2}=$ $4^{-\frac{2 \sigma+1}{\sigma-1}-1} \alpha$. Note that the corresponding positive solution $u_{\alpha_{2}}$ is strictly below $u_{\alpha_{1}}=u_{\alpha}$. In this way we obtain the required sequence of solutions. If $h \neq \equiv \bar{h}$, we 
reason as in Remark 4.33. Let $y$ be a solution of (4.98). Then, by Sturm comparison $\bar{h} \leq y \leq h$, thus the validity of (4.95) implies the validity of the analogous of (4.95) with $y$ replacing $h$. Applying the previous proof we get a sequence of solutions $u_{j}$ such that

$$
C_{1, j} \bar{h}(r(x)) \leq C_{1, j} y(r(x)) \leq u_{j}(x) \leq C_{2, j} y(r(x)) \leq C_{2, j} h(r(x))
$$

on $M_{g}$. This completes the proof of the theorem.

To better appreciate the above theorem, we give two geometric applications. Firstly, we deduce Theorem 4.29 as an immediate corollary.

Proof of Theorem 4.29. Defining $u$ as in (2.54), $u$ must be a positive solution of (2.55), which on Euclidean space reads as

$$
\Delta u+\frac{\widetilde{s}(x)}{c_{m}} u^{\frac{m+2}{m-2}}=0, \quad \text { where } c_{m}=\frac{4(m-1)}{m-2} .
$$

Set $\sigma=(m+2) /(m-2), b(x)=-\widetilde{s}(x) / c_{m}$, and realize $\mathbb{R}^{m}$ as a model manifold with $g(r)=r$, so that $\chi(r)=(m-2)^{2} /\left(4 r^{2}\right)$. To apply Theorem 4.35, we choose $A \equiv 0, h=\bar{h}=1$ and $k>0$ small enough in such a way that $2+(m-2) \frac{1-\sqrt{1-k}}{2}<l$. This is possible since $l>2$. Then, $|b(x)| \leq C r^{-l}$ implies the inequality

$$
|b(x)| \leq C r(x)^{-2-(m-2) \frac{1-\sqrt{1-k}}{2}},
$$

which is (4.95) in our setting. Thus, the existence of the desired conformal deformations follows from Theorem 4.35.

Secondly, specializing the main theorem to the hyperbolic setting, we get the following extension of $\mathrm{Ni}$ result.

Corollary 4.36. Consider the hyperbolic space $\mathbb{H}_{B}^{m}$ of sectional curvature $-B^{2}$ and dimension $m \geq 3$, and let $\widetilde{s}(x) \in C^{\infty}\left(\mathbb{H}_{B}^{m}\right)$ be a function satisfying

$$
|\widetilde{s}(x)| \leq C e^{(2 B-\delta) r(x)},
$$

for some $\delta>0$ and for some constant $C>0$. Then, for every $\varepsilon>0$, the Poincaré metric $\langle$,$\rangle can be conformally deformed to a smooth metric \overline{\langle,\rangle}$ of scalar curvature $\widetilde{s}$ and satisfying

$$
C_{1} e^{-(2 B+\varepsilon) r(x)}\langle,\rangle_{x} \leq \widetilde{\langle,\rangle_{x}} \leq C_{2} e^{-(2 B-\varepsilon) r(x)}\langle,\rangle_{x} \quad \forall x \in \mathbb{H}_{B}^{m},
$$

for some $0<C_{1} \leq C_{2}$. Furthermore, for each fixed $\varepsilon>0, C_{1}$ and $C_{2}$ and can be chosen to be as small as we wish.

Proof. The scalar curvature of $\mathbb{H}_{B}^{m}$ is $-m(m-1) B^{2}$, thus the conformal factor $u$ in (2.54) has to satisfy

$$
\Delta u+\frac{B^{2} m(m-2)}{4} u+\frac{\widetilde{s}(x)}{c_{m}} u^{\frac{m+2}{m-2}}=0, \quad \text { where } \quad c_{m}=\frac{4(m-1)}{m-2} .
$$

Set $\sigma=(m+2) /(m-2), b(x)=-\widetilde{s}(x) / c_{m}, A(r)=B^{2} m(m-2) / 4$, and realize $\mathbb{H}_{B}^{m}$ as a model manifold with $g(r)=B^{-1} \sinh (B r)$. Hence, $v(r)=g(r)^{m-1}$ satisfies $\left(\mathrm{V}_{\mathrm{L} 1}\right)$ and, by Remark 3.19 and Proposition 3.23, for every $r \in \mathbb{R}^{+}$

$$
\chi(r)>\frac{B^{2}(m-1)^{2}}{4} \quad \text { and } \quad \chi(r) \rightarrow \frac{B^{2}(m-1)^{2}}{4} \text { as } r \rightarrow+\infty .
$$


We let $k_{1}=\frac{m(m-2)}{(m-1)^{2}}<1$. Note that $k_{1}$ is the smallest $k$ for which

$$
\frac{m(m-2) B^{2}}{4}=A(r)<k_{1} \chi(r) \quad \text { on } \mathbb{R}^{+}
$$

For $k_{0}<k_{1}, k_{0} \chi(r) \rightarrow k_{0} \frac{m-1}{4} B^{2}<A(r)$. Hence there exists $r_{1}=r_{1}\left(k_{0}\right)>>1$ such that on $\left[r_{1},+\infty\right), k_{0} \chi(r)<A(r)$. We define $\bar{A}$ on $\mathbb{R}_{0}^{+}$so that $\bar{A} \leq A$ on $\mathbb{R}_{0}^{+}$ and $\overline{A(r)}=k_{0} \chi(r)$ on $\left[r_{1},+\infty\right)$. We let $h$ be the solution of

$$
\left\{\begin{array}{l}
\left(v h^{\prime}\right)^{\prime}+\bar{A} v h=0 \quad \text { on } \mathbb{R}^{+} \\
h(0)=1, \quad h^{\prime}(0)=0 .
\end{array}\right.
$$

Then, by Theorem 4.1 and Proposition $4.7, h>0$ on $\mathbb{R}^{+}$and

$$
h(r) \asymp \exp \left\{-\frac{B(m-1)\left(1-\sqrt{1-k_{0}}\right)}{2} r\right\} \quad \text { as } r \rightarrow+\infty
$$

Moreover, since $\bar{A} \leq A, h$ solves the first of (4.86) and can therefore be used to verify condition (4.95) in Theorem 4.35. Choose $k \in\left(k_{1}, 1\right)$. Then $A<k \chi(r)$ on $\mathbb{R}_{0}^{+}$and

$$
\left[\int_{r}^{+\infty} \frac{\mathrm{d} s}{v(s)}\right]^{(1-\sqrt{1-k}) / 2} \asymp \exp \left\{-\frac{B(m-1)(1-\sqrt{1-k})}{2} r\right\} \quad \text { as } r \rightarrow+\infty .
$$

Thus, for $\sigma=\frac{m+2}{m-2}$,

$$
\frac{k \chi(r)-A(r)}{h^{\sigma}(r)} \geq \frac{\left(k-k_{1}\right) \chi(r)}{h^{\sigma}(r)} \geq C \exp \left\{\frac{\sigma}{2} B(m-1)\left(1-\sqrt{1-k_{0}}\right) r\right\}
$$

for some constant $C>0$ depending on $k, k_{1}$. This implies that (4.95) is satisfied if on $\mathbb{H}_{B}^{m}$

$$
|\widetilde{s}(x)|=c_{m}|b(x)| \leq C \exp \left\{\frac{B(m-1)}{2}\left[\sigma\left(1-\sqrt{1-k_{0}}\right)-(1-\sqrt{1-k})\right] r\right\}
$$

for some constant $C>0$. Next we observe that for

$$
k_{0} \uparrow k_{1}, \quad \frac{B(m-1)}{2} \sigma\left(1-\sqrt{1-k_{0}}\right) \rightarrow \sigma \frac{B(m-2)}{2}=B \frac{m+2}{2}
$$

and for

$$
k \downarrow k_{1}, \quad \frac{B(m-1)}{2}(1-\sqrt{1-k}) \rightarrow B \frac{m-2}{2}
$$

We can therefore choose $k_{0}$ and $k$ sufficiently close to $k_{1}$ so that (4.109)

$$
\begin{aligned}
& \frac{1}{2} B(m-1)\left(1-\sqrt{1-k_{0}}\right) \geq B \frac{m-2}{2}-\varepsilon \frac{m-2}{4} \\
& \frac{1}{2} B(m-1)(1-\sqrt{1-k}) \leq B \frac{m-2}{2}+\varepsilon \frac{m-2}{4} \\
& 2 B-\delta=B \frac{m+2}{2}-B \frac{m-2}{2}-\delta \leq \frac{B}{2}(m-1)\left[\sigma\left(1-\sqrt{1-k_{0}}\right)-(1-\sqrt{1-k})\right]
\end{aligned}
$$

Thus (4.104) implies the validity of (4.108) and therefore of (4.95). Applying Theorem 4.35 we get the existence of infinitely many solutions $u$ of (4.106) such that

$$
C_{1} e^{-\frac{B(m-1)(1-\sqrt{1-k})}{2} r(x)} \leq u(x) \leq C_{2} e^{-\frac{B(m-1)\left(1-\sqrt{1-k_{0}}\right)}{2} r(x)} \quad \text { on } \mathbb{H}_{B}^{m},
$$


where $C_{1}, C_{2}$ can be chosen as small as we wish. From (4.109), we deduce

$$
C_{1} e^{-\left(\frac{B(m-2)}{2}+\varepsilon \frac{m-2}{4}\right) r(x)} \leq u(x) \leq C_{2} e^{-\left(\frac{B(m-2)}{2}-\varepsilon \frac{m-2}{4}\right) r(x)} \quad \text { on } \mathbb{H}_{B}^{m} .
$$

Since $\widetilde{\langle,\rangle}=u^{\frac{4}{m-2}}\langle$,$\rangle , we get the desired (4.105).$

REMARK 4.37. The lower bound in (4.110) is sharp. In this respect, see the discussion after Corollary 4.28, Theorem 4.23 and Remark 4.27.

REMARK 4.38. The estimates for $|b(x)|$ in Theorem 4.35 are sharp. Indeed, in the hyperbolic setting, by Corollary B of [BR97] no conformal deformations of $\mathbb{H}_{B}^{m}$ exist if we require that

$$
\widetilde{s}(x) \leq-C \frac{e^{2 B r(x)}}{r(x) \log r(x)} \quad \text { for } r(x)>>1,
$$

which shows the necessity of $\delta>0$ in (4.104). In the spirit of Naito improvement of Ni result, this motivates the next

\section{Conjectures:}

(1) The Poincaré metric can be conformally deformed to a new metric of scalar curvature $\widetilde{s}(x)$ provided that there exists $S(r)$ such that

$$
|\widetilde{s}(x)| \leq S(r(x)) \quad \text { and } \quad e^{-2 B r} S(r) \in L^{1}(+\infty) .
$$

(2) If

$$
\widetilde{s}(x) \leq-S(r(x)) \leq 0 \quad \text { and } \quad e^{-2 B r} S(r) \notin L^{1}(+\infty),
$$

then the Poincaré metric cannot be conformally deformed to a new metric of scalar curvature $\widetilde{s}(x)$.

We mention that, in the forecoming paper $[\mathbf{B M R}]$, we improve and complement the results of this section and we move some steps towards the solution of these conjectures.

\subsection{Upper bounds for the number of zeroes of $z$}

Once we know that the number of zeroes of $z$ solving (4.3) is finite, say, $n$ (for instance, this is always the case when $z$ comes from the radialization of an operator $L=-\Delta-q(x)$ with finite index), the next step is to determine upper bounds for $n$. In passing, we note that, by classical Sturm-Liouville theory ([Wei87], Theorem 14.2), $n$ is also the index of the self-adjoint extension of the operator

$$
L=-v^{-1} \frac{\mathrm{d}}{\mathrm{d} r}\left(v \frac{\mathrm{d}}{\mathrm{d} r}\right)+A \quad \text { on } C_{c}^{\infty}\left(\mathbb{R}_{0}^{+}, v \mathrm{~d} r\right) .
$$

This section rests upon some ideas in a recent paper of T. Ekholm, R.L. Frank and H. Kovařì, [EFK11], in which upper bounds for the index of Schrödinger operators on metric trees are derived from inequalities on the corresponding radialized ODE. The analytical core is the following weighted Hardy-Sobolev inequality.

Theorem 4.39 ([OK90], Theorem 6.2). Let $2 \leq q \leq+\infty, \xi, \eta \in L_{\text {loc }}^{\infty}\left(\mathbb{R}_{0}^{+}\right)$. Then, the inequality

$$
\left(\int_{0}^{+\infty}|\eta(r) u(r)|^{q} \mathrm{~d} r\right)^{2 / q} \leq S^{2} \int_{0}^{+\infty}\left|\xi(r) u^{\prime}(r)\right|^{2} \mathrm{~d} r
$$


holds, for some $S>0$ and for every absolutely continuous $u$ such that $u(r) \rightarrow 0$ as $r \rightarrow+\infty$ if and only if

$$
T=\sup _{r>0}\left\{\|\eta\|_{L^{q}([0, r])}\|1 / \xi\|_{L^{2}([r,+\infty))}\right\}<+\infty .
$$

If this is the case, the best constant $S$ satisfies

$$
\begin{array}{ll}
T \leq S \leq T\left(1+\frac{q}{2}\right)^{1 / q}\left(1+\frac{2}{q}\right)^{1 / 2} & \text { if } q<+\infty \\
S=T & \text { if } q=+\infty .
\end{array}
$$

A direct application of Theorem 4.39 gives

TheOrem 4.40 ([EFK11], Theorem 2.15). Let A, v satisfy assumptions (A1), (V1), $\left(\mathrm{V}_{\mathrm{L} 1}\right)$, and let $z$ be a $\mathrm{Lip}_{\mathrm{loc}}$ solution of (4.3). Let $\left\{z_{j}\right\}_{j=1}^{n}$ be the zeroes of $z, n \leq+\infty$. Let $w: \mathbb{R}^{+} \rightarrow \mathbb{R}^{+}$be an integrable function such that, for some $2<q \leq+\infty$

$$
\begin{array}{ll}
C=\sup _{r>0}\left[\left(\int_{0}^{r} v(s)^{\frac{q}{2}} w(s)^{-\frac{q-2}{2}} \mathrm{~d} s\right)^{2 / q} \int_{r}^{+\infty} \frac{\mathrm{d} s}{v(s)}\right]<+\infty & \text { if } q<+\infty ; \\
C=\sup _{r>0}\left[\left(\sup _{s \in[0, r]} \frac{v(s)}{w(s)}\right) \int_{r}^{+\infty} \frac{\mathrm{d} s}{v(s)}\right]<+\infty & \text { if } q=+\infty .
\end{array}
$$

Set $p=q /(q-2)$ if $q<+\infty, p=1$ if $q=+\infty$. Then, there exists an optimal constant $N_{p}(w)>0$ such that

$$
n \leq N_{p}(w) \int_{0}^{+\infty} A_{+}(r)^{p} w(r) \mathrm{d} r .
$$

Furthermore, $N_{p}(w)$ satisfies

$$
\begin{array}{ll}
N_{p}(w) \leq\left(1+p^{\prime}\right)^{p-1}\left(1+\frac{1}{p^{\prime}}\right)^{p} C^{p} & \text { if } q<+\infty ; \\
N_{p}(w) \leq C & \text { if } q=+\infty,
\end{array}
$$

where $p^{\prime}=p /(p-1)=q / 2$.

Proof. We consider the case $q<+\infty$, the remaining case being simpler. Because of (4.113) we can apply the Hardy-Sobolev inequality of Theorem 4.39 with the choice

$$
\xi(r)=\sqrt{v(r)}, \quad \eta(r)=v(r)^{\frac{1}{2}} w(r)^{-\frac{q-2}{2 q}}
$$

to deduce

$$
\left(\int_{0}^{+\infty} v(s)^{\frac{q}{2}} w(s)^{-\frac{q-2}{2}}|u(s)|^{q} \mathrm{~d} s\right)^{2 / q} \leq S^{2} \int_{0}^{+\infty}\left|u^{\prime}(s)\right|^{2} v(s) \mathrm{d} s
$$

for every $u$ with compact support in $\mathbb{R}_{0}^{+}$, where

$$
\begin{array}{ll}
\sqrt{C} \leq S \leq \sqrt{C}\left(1+\frac{q}{2}\right)^{1 / q}\left(1+\frac{2}{q}\right)^{1 / 2} & \text { if } q<+\infty ; \\
S=\sqrt{C} & \text { if } q=+\infty .
\end{array}
$$


Let $u=z \chi_{\left[z_{j-1}, z_{j}\right]}$, where $z_{0}=0$. Then, integrating by parts and using Hölder inequality with conjugate exponents $p$ and $p^{\prime}=q / 2$ we get

$$
\begin{aligned}
& \left(\int_{z_{j-1}}^{z_{j}} v(s)^{\frac{q}{2}} w(s)^{-\frac{q-2}{2}}|z(s)|^{q} \mathrm{~d} s\right)^{2 / q} \leq S^{2} \int_{z_{j-1}}^{z_{j}}\left(z^{\prime}(s)\right)^{2} v(s) \mathrm{d} s \\
& =S^{2} \int_{z_{j-1}}^{z_{j}} A(s) v(s)|z(s)|^{2} \mathrm{~d} s \leq S^{2} \int_{z_{j-1}}^{z_{j}} A_{+}(s) v(s)|z(s)|^{2} \mathrm{~d} s \\
& \leq S^{2}\left(\int_{z_{j-1}}^{z_{j}} w(s)^{-\frac{q-2}{2}} v(s)^{\frac{q}{2}}|z(s)|^{q} \mathrm{~d} s\right)^{2 / q}\left(\int_{z_{j-1}}^{z_{j}} A_{+}(s)^{p} w(s) \mathrm{d} s\right)^{1 / p}
\end{aligned}
$$

Simplifying and taking the $p$-th power we obtain

$$
1 \leq S^{2 p} \int_{z_{j-1}}^{z_{j}} A_{+}(s)^{p} w(s) \mathrm{d} s .
$$

Estimate (4.114) and the bound (4.115) follow at once summing up with respect to $j$.

Clearly, it would be nice to find suitable functions $w$ such that conditions (4.113) are automatically satisfied. The problem is addressed in the following

Corollary 4.41. Let $A, v, z,\left\{z_{j}\right\}_{j=1}^{n}$ be as in the previous theorem, and let $\chi$ be the critical curve. Assume also that $1 / v \notin L^{1}\left(0^{+}\right)$. Then, for every fixed $p \in[1,+\infty)$

$$
n \leq\left(\frac{2 p-1}{2 p}\right)^{2 p-1} \int_{0}^{+\infty}\left[\frac{1}{\sqrt{\chi(s)}}\right]^{2 p-1} A_{+}(s)^{p} \mathrm{~d} s
$$

Proof. We begin with the case $p>1$. Let $q$ be such that $p=q /(q-2)<+\infty$. To apply the previous theorem, we will find $w(r)$ such that

$$
\left(\int_{0}^{r} v(s)^{\frac{q}{2}} w(s)^{-\frac{q-2}{2}} \mathrm{~d} s\right)^{2 / q} \int_{r}^{+\infty} \frac{\mathrm{d} s}{v(s)}=1 \quad \text { on } \mathbb{R}^{+},
$$

so that $C=1$. An algebraic manipulation with the aid of the initial condition $1 / v \notin L^{1}\left(0^{+}\right)$and the definition of $\chi$ gives

$$
w(r)=2^{-\frac{q}{q-2}} q^{-\frac{2}{q-2}}\left(\frac{1}{\sqrt{\chi(r)}}\right)^{\frac{q+2}{q-2}} .
$$

An application of Theorem 4.40 taking into account the upper bound (4.115) yields

$$
n \leq\left(\frac{q+2}{2 q}\right)^{\frac{q+2}{q-2}} \int_{0}^{+\infty}\left[\frac{1}{\sqrt{\chi(s)}}\right]^{\frac{q+2}{q-2}} A_{+}(s)^{\frac{q}{q-2}} \mathrm{~d} s
$$

Rewriting with respect to $p$ we get (4.116). The case $p=1$ is obtained by setting $q=+\infty$. The choice

implies

$$
w(r)=\frac{1}{2 \sqrt{\chi(r)}}
$$

$$
\left(\sup _{s \in[0, r]} \frac{v(s)}{w(s)}\right) \int_{r}^{+\infty} \frac{\mathrm{d} s}{v(s)}=1 \quad \text { on } \mathbb{R}^{+},
$$


hence $C=1$ and (4.116) follows at once from the definition of $w(r),(4.114)$ and (4.115).

Despite their simplicity, it should be stressed that, in some unfortunate circumstances, (4.116) is not sharp. Indeed, assume that $A \leq \chi$ on $\mathbb{R}^{+}$and $A=\chi$ on $\left[r_{0},+\infty\right)$, for some $r_{0}>0$. Then, after $r_{0}$ the integrand in estimate (4.116) is $\sqrt{\chi(s)}$, which is non-integrable by (3.23). However, as we saw in Proposition 4.1, $n=0$ and (4.116) is useless.

By means of the change of variables in Proposition 3.10, we can also give a corresponding statement for solutions $g$ of $g^{\prime \prime}+K(s) g=0$.

Corollary 4.42. Let $K \in L_{\text {loc }}^{\infty}\left(\mathbb{R}_{0}^{+}\right)$, and let $g$ be a solution of

$$
\left\{\begin{array}{l}
g^{\prime \prime}+K(s) g=0 \quad \text { on } \mathbb{R}^{+}, \\
g(0)=0, \quad g^{\prime}(0)=1 .
\end{array}\right.
$$

Then, for every $p \in[1,+\infty)$, the number of zeroes $n$ of $g$ satisfies

$$
n \leq\left(\frac{2 p-1}{2 p}\right)^{2 p-1} \int_{0}^{+\infty} \sigma^{2 p-1} K_{+}(\sigma)^{p} \mathrm{~d} \sigma
$$

We note that the role of the critical curve $\chi$ seems to be ubiquitous in deriving the validity of Hardy-Sobolev inequalities like that of Theorem 4.39, although sometimes there is no evidence of it in the formulas. Corollaries 4.41 and 4.42 are simple examples. In this respect, the treatise [OK90] is a wealth of information.

On the links between $\chi$ and $\tilde{\chi}$, II. Before proceeding, we would like to make a few further observations on the relation between $\chi$ and $\tilde{\chi}$ discussed at the end of Section 4.2. We proceed with a reasoning for the $m \geq 3$ dimensional case. Our task is to see whether $\tilde{\chi}$ can replace $\chi$ in the uncertainty principle lemma, that is, in Theorem 4.15, and furthermore if this replacement gives a better result.

With the aid of Theorem 4.39, we obtain the following necessary condition on $\tilde{\chi}$ to be a critical curve on a model manifold.

Proposition 4.43. Let $\left(M_{g}, \mathrm{~d} s^{2}\right)$ be an m-dimensional model with metric given, in polar coordinates, by $\mathrm{d} s^{2}=\mathrm{d} r^{2}+g(r)^{2} \mathrm{~d} \theta^{2}$. Suppose that $m \geq 3$, and set $f(r)=g(r)^{m-1}$. If the uncertainty principle lemma

$$
\int_{M} \tilde{\chi}_{f}(r(x)) u^{2}(x) \mathrm{d} V(x) \leq \int_{M}|\nabla u(x)|^{2} \mathrm{~d} V(x)
$$

holds for every $u \in \operatorname{Lip}_{c}(M)$, with $\widetilde{\chi}_{f}=\left[f^{\prime} /(2 f)\right]^{2}$ the modified critical function, then

$$
\inf _{r>0}\left(2 r \sqrt{\chi_{f}(r)}\right) \geq 1
$$

where $\chi_{f}(r)$ is the critical function of $f(r)$.

Proof. By restricting (4.119) to radial, compactly supported Lipschitz test functions $u(r(x))$, the following inequality holds for every $u \in \operatorname{Lip}_{c}\left(\mathbb{R}_{0}^{+}\right)$:

$$
\int_{0}^{+\infty}\left(\frac{m-1}{2} \frac{g(s)^{\prime}}{g(s)}\right)^{2} u^{2}(s) g(s)^{m-1} \mathrm{~d} s \leq \int_{0}^{+\infty}\left(u^{\prime}(s)\right)^{2} g(s)^{m-1} \mathrm{~d} s .
$$

Applying Theorem 4.39 with the choices

$$
q=2, \quad S \leq 1, \quad \xi(r)=g(r)^{\frac{m-1}{2}}, \quad \eta(r)=\frac{m-1}{2} \frac{g(r)^{\prime}}{g(r)} g(r)^{\frac{m-1}{2}}=\xi^{\prime}(r)
$$


by estimate (4.112), the validity of (4.120) forces the inequality $T^{2} \leq 1$, where

$$
T^{2}=\sup _{r>0} T^{2}(r) \quad \text { and } T^{2}(r)=\left(\int_{0}^{r}\left[\left(g(s)^{\frac{m-1}{2}}\right)^{\prime}\right]^{2} \mathrm{~d} s\right)\left(\int_{r}^{+\infty} \frac{\mathrm{d} s}{g(s)^{m-1}}\right),
$$

for otherwise the sharp constant $S$ in (4.111) would be strictly greater than 1 . Note that, through a standard approximation procedure, (4.111) holds for every absolutely continuous $u$ converging to zero at infinity if and only if it holds for every $u \in \operatorname{Lip}_{c}\left(\mathbb{R}_{0}^{+}\right)$. By the Cauchy-Schwarz inequality and the definition of the critical curve $(3.21)$ for $v(r)=f(r)$, we deduce

$$
T^{2}(r) \geq \frac{g(r)^{m-1}}{r} \int_{r}^{+\infty} \frac{\mathrm{d} s}{g(s)^{m-1}}=\frac{1}{2 r \sqrt{\chi_{f}(r)}}
$$

and combining with $T^{2} \leq 1$ we get the desired inequality.

It is worth to observe that, with the choice (4.121), inequality (4.120) has the expression

$$
\int_{0}^{+\infty}\left(\xi^{\prime}\right)^{2} u^{2} \leq \int_{0}^{+\infty} \xi^{2}\left(u^{\prime}\right)^{2} \quad \forall u \in \operatorname{Lip}_{c}\left(\mathbb{R}_{0}^{+}\right)
$$

This is often called a (1-dimensional) Caccioppoli inequality. By a standard technique, which we now briefly recall, if $\xi$ is non-negative and convex (and this is often the case by its very definition and the properties of $g$ ), (4.123) holds up to a factor of 4 . As a consequence of the estimates (4.112) of the sharp constant in Theorem 4.39, this means that $T<+\infty$, where $T$ is as in (4.122). Without loss of generality, we can limit ourselves to prove Caccioppoli inequality with $u$ compactly supported in $\mathbb{R}_{0}^{+}$. We integrate $\xi^{\prime \prime}\left(\xi u^{2}\right) \geq 0$ by parts, we use Young inequality and $\xi \in C^{\infty}\left(\mathbb{R}_{0}^{+}\right), \xi(0)=0$ to get, for every $\delta \in(0,1)$,

$$
\begin{aligned}
0 & \leq \int_{0}^{+\infty} \xi^{\prime \prime} \xi u^{2}=-\int_{0}^{+\infty} \xi^{\prime}\left(\xi u^{2}\right)^{\prime}=-\int_{0}^{+\infty}\left(\xi^{\prime}\right)^{2} u^{2}-\int_{0}^{+\infty} 2 \xi \xi^{\prime} u u^{\prime} \\
& \leq(\delta-1) \int_{0}^{+\infty}\left(\xi^{\prime}\right)^{2} u^{2}+\frac{1}{\delta} \int_{0}^{+\infty} \xi^{2}\left(u^{\prime}\right)^{2},
\end{aligned}
$$

hence

$$
\int_{0}^{+\infty}\left(\xi^{\prime}\right)^{2} u^{2} \leq\left[\inf _{\delta \in(0,1)} \frac{1}{\delta(1-\delta)}\right] \int_{0}^{+\infty} \xi^{2}\left(u^{\prime}\right)^{2}=4 \int_{0}^{+\infty} \xi^{2}\left(u^{\prime}\right)^{2},
$$

as desired. 


\section{CHAPTER 5}

\section{Exceeding the critical curve}

In this Chapter we give some sufficient conditions to guarantee that a solution $z \in \operatorname{Lip}_{\text {loc }}\left(\mathbb{R}_{0}^{+}\right)$of the problem

$$
\left\{\begin{array}{l}
\left(v(r) z^{\prime}(r)\right)^{\prime}+A(r) v(r) z(r)=0 \quad \text { on } \mathbb{R}^{+}, \\
z(0)=z_{0}>0
\end{array}\right.
$$

either has a first zero or it is oscillatory. One of the main features of our results is that we do not require $A(r)$ to be non-negative. However, the case $A(r) \geq 0$ is more transparent, easier to handle and sufficient for some geometric applications. For this reason, we first deal with $A \geq 0$. Throughout this section we shall also consider a bounding function $f$ defined on $\mathbb{R}_{0}^{+}$and satisfying the following requirements:

$$
f \in L_{\mathrm{loc}}^{\infty}\left(\mathbb{R}_{0}^{+}\right), \quad \frac{1}{f} \in L_{\mathrm{loc}}^{\infty}\left(\mathbb{R}^{+}\right), \quad 0 \leq v \leq f \quad \text { on } \mathbb{R}_{0}^{+} .
$$

\subsection{First zero and oscillation}

The techniques used in the proof of the next theorem remind some in the work of M.P. Do Carmo and D. Zhou [CZ99]. Observe that assumptions (A1) and (V1) have been introduced in Section 3.1.

Theorem 5.1. Let (A1), (V1), (F1) be met, and assume that $z \in \operatorname{Lip}_{\text {loc }}\left(\mathbb{R}_{0}^{+}\right)$ is a positive solution of

$$
\left\{\begin{array}{l}
\left(v(r) z^{\prime}(r)\right)^{\prime}+A(r) v(r) z(r)=0 \quad \text { on } \mathbb{R}^{+}, \\
z(0)=z_{0}>0
\end{array}\right.
$$

Suppose $A \geq 0$ and $A \not \equiv 0$. Then

$$
\frac{1}{v(r)} \in L^{1}(+\infty)
$$

and for every $0<R_{0}<r$ such that $A \not \equiv 0$ in $L^{\infty}\left(\left[0, R_{0}\right]\right)$

$$
\int_{R_{0}}^{r}\left(\sqrt{A(s)}-\sqrt{\chi_{f}(s)}\right) \mathrm{d} s \leq-\frac{1}{2}\left(\log \int_{0}^{R_{0}} A(s) v(s) \mathrm{d} s+\log \int_{R_{0}}^{+\infty} \frac{\mathrm{d} s}{f(s)}\right) .
$$

Proof. We define

$$
y(r)=-\frac{v(r) z^{\prime}(r)}{z(r)} \in \operatorname{Lip}_{\mathrm{loc}}\left(\mathbb{R}_{0}^{+}\right) .
$$

Because of (5.2) and (V1), $y$ satisfies Riccati equation

$$
y^{\prime}=A v+\frac{y^{2}}{v} \quad \text { a.e. on } \mathbb{R}^{+}, \quad \text { with } y(0)=0 .
$$


Since $A \geq 0, y^{\prime} \geq 0$ a.e. on $\mathbb{R}^{+}$and, for every $R_{0}>0$ such that $A \not \equiv 0$ on $\left[0, R_{0}\right]$

$$
y(r) \geq y\left(R_{0}\right) \geq \int_{0}^{R_{0}} A(s) v(s) \mathrm{d} s>0 \quad \forall r \in\left[R_{0},+\infty\right)
$$

Using (5.6) and Young inequality $\varepsilon a^{2}+\varepsilon^{-1} b^{2} \geq 2|a||b|, a, b \in \mathbb{R}, \varepsilon>0$ we also deduce

$$
y^{\prime} \geq 2 y \sqrt{A} \quad \text { a.e. on }\left[R_{0},+\infty\right)
$$

From (5.7) and (5.8) we infer

$$
y(r) \geq\left(\int_{0}^{R_{0}} A(s) v(s) \mathrm{d} s\right) \exp \left\{2 \int_{R_{0}}^{r} \sqrt{A(s)} \mathrm{d} s\right\} \quad \text { on }\left[R_{0},+\infty\right)
$$

Moreover, from (5.6),

$$
\frac{y^{\prime}}{y^{2}} \geq \frac{1}{v} \quad \text { a.e. on }\left[R_{0},+\infty\right)
$$

and integrating on $[r, R]$ we get

$$
\frac{1}{y(r)} \geq \frac{1}{y(R)}+\int_{r}^{R} \frac{\mathrm{d} s}{v(s)} \geq \int_{r}^{R} \frac{\mathrm{d} s}{v(s)} .
$$

Letting $R \rightarrow+\infty$ we obtain (5.3), and using (5.11) into (5.9) we reach the following inequality:

$$
\int_{R_{0}}^{r} \sqrt{A(s)} \mathrm{d} s \leq-\frac{1}{2} \log \int_{0}^{R_{0}} A(s) v(s) \mathrm{d} s-\frac{1}{2} \log \int_{r}^{R} \frac{\mathrm{d} s}{v(s)} .
$$

Letting $R \rightarrow+\infty$, inequality (5.4) is simply a rewriting of (5.12). Indeed, by (F1) and (3.22) for $\chi_{f}(r)$

$$
-\frac{1}{2} \log \int_{r}^{+\infty} \frac{\mathrm{d} s}{v(s)} \leq-\frac{1}{2} \log \int_{r}^{+\infty} \frac{\mathrm{d} s}{f(s)}=-\frac{1}{2} \log \int_{R_{0}}^{+\infty} \frac{\mathrm{d} s}{f(s)}+\int_{R_{0}}^{r} \sqrt{\chi_{f}(s)} \mathrm{d} s .
$$

COROllary 5.2 (First zero). In the assumptions of the previous theorem, let $A \geq 0, A \not \equiv 0$. Suppose that either $1 / f \notin L^{1}(+\infty)$ or otherwise there exist $0<R_{0}<r$ such that $A \not \equiv 0$ on $\left[0, R_{0}\right]$ and

$$
\int_{R_{0}}^{r}\left(\sqrt{A(s)}-\sqrt{\chi_{f}(s)}\right) \mathrm{d} s>-\frac{1}{2}\left(\log \int_{0}^{R_{0}} A(s) v(s) \mathrm{d} s+\log \int_{R_{0}}^{+\infty} \frac{\mathrm{d} s}{f(s)}\right)
$$

Then, the solution $z \in \operatorname{Lip}_{\text {loc }}\left(\mathbb{R}_{0}^{+}\right)$of (5.2) has a first zero. Moreover, in this second case, this is attained on $(0, \bar{R}]$, where $\bar{R}>0$ is the unique real number satisfying

$$
\int_{R_{0}}^{r} \sqrt{A(s)} \mathrm{d} s=-\frac{1}{2} \log \int_{0}^{R_{0}} A(s) v(s) \mathrm{d} s-\frac{1}{2} \log \int_{r}^{\bar{R}} \frac{\mathrm{d} s}{f(s)} .
$$

Proof. When $1 / f \notin L^{1}(+\infty)$, the existence of a first zero for $z$ is immediate from Theorem 5.1. Suppose now that $1 / f \in L^{1}(+\infty)$. Then (5.14) is equivalent to say that (5.4) is false for some $0<R_{0}<r$. Hence, $z$ has again a first zero thanks to Theorem 5.1. 
As for its positioning, we first note that (5.4) comes from (5.12), that we write as

$$
\int_{R_{0}}^{r} \sqrt{A(s)} \mathrm{d} s \leq-\frac{1}{2}\left(\log \int_{0}^{R_{0}} A(s) v(s) \mathrm{d} s+\log \int_{r}^{R} \frac{\mathrm{d} s}{f(s)}\right) \quad \forall R>r
$$

and valid for $R$ before the first zero of $z$. Let $H(R)$ denote the RHS of the above, which may be thought to be defined on $(r,+\infty)$. Note that $H$ is continuous, strictly decreasing for $R \in(r,+\infty)$, and $H(R) \rightarrow+\infty$ as $R \rightarrow r^{+}$. Using (3.22), we rewrite (5.14) as

$$
\int_{R_{0}}^{r} \sqrt{A(s)} \mathrm{d} s>-\frac{1}{2}\left(\log \int_{0}^{R_{0}} A(s) v(s) \mathrm{d} s+\log \int_{r}^{+\infty} \frac{\mathrm{d} s}{f(s)}\right) .
$$

Comparing the last two inequalities, we deduce the existence of a unique $\bar{R}>r$ such that (5.15) holds. For every $\varepsilon>0$, Theorem 5.1 gives the existence of a first zero on $(0, \bar{R}+\varepsilon)$, so that letting $\varepsilon \rightarrow 0$ we reach the desired conclusion.

REMARK 5.3. In case $1 / f \notin L^{1}(+\infty)$, by slightly modifying the above proof it can be shown that the solution $\bar{R}$ of (5.15) still gives an upper bound for the first zero of $z$. Note that, in (5.15), the only requirement on $R_{0}$ is that $A \not \equiv 0$ on $\left[0, R_{0}\right]$.

As already underlined in Example 4.9, (2), inequality (5.14) is deep since the right hand side of (5.14) is independent both of $r$ and of the behavior of $A$ after $R_{0}$ : if (5.4) is contradicted for some $0<R_{0}<r<R$, the left hand side represents how much must $A$ exceed a critical curve modelled on $f$ in the compact region $\left[R_{0}, r\right]$ in order to have a first zero of $z$, and it only depends on the behavior of $A$ and $f$ before $R_{0}$ (the first addendum of the RHS), and on the growth of $f$ after $R_{0}$.

REMARK 5.4. It is worth observing that, in order to obtain (5.14), we need to assume $A \geq 0$ on the whole $\mathbb{R}^{+}$and not, a posteriori, only on $(0, \bar{R})$.

REMARK 5.5. The assumptions of Theorem 5.1 and Corollary 5.2 can be weakened. Indeed, the reader can check that all the reasonings in both proofs are still valid even if $z$ satisfies the differential inequality

$$
\left(v z^{\prime}\right)^{\prime}+A v z \leq 0,
$$

provided that the initial condition is such that

$$
y\left(0^{+}\right)=-\frac{v z^{\prime}}{z}\left(0^{+}\right)=0
$$

(see inequality (5.7)). In particular, a mild singularity of $z$ as $r \rightarrow 0^{+}$is allowed if $v(r)$ tends to zero sufficiently fast. This will be useful in Section 5.7.

Theorem 5.6 (Oscillation). Assume that (A1), (V1), (F1), $A \geq 0$ hold on $\left[r_{0},+\infty\right)$, for some $r_{0} \geq 0$. Let $z_{0} \in \mathbb{R} \backslash\{0\}$. Suppose that either

$$
\frac{1}{f(r)} \notin L^{1}(+\infty) \quad \text { and } \quad A(r) v(r) \notin L^{1}(+\infty)
$$

or

$$
\frac{1}{v(r)} \in L^{1}(+\infty) \quad \text { and } \quad \limsup _{r \rightarrow+\infty} \int_{R}^{r}\left(\sqrt{A(s)}-\sqrt{\chi_{f}(s)}\right) \mathrm{d} s=+\infty
$$


for some (hence any) $R>r_{0}$. Then, every solution $z(r) \in \operatorname{Lip}_{\text {loc }}\left(\left[r_{0},+\infty\right)\right)$ of

$$
\left\{\begin{array}{l}
\left(v(r) z^{\prime}(r)\right)^{\prime}+A(r) v(r) z(r)=0 \quad \text { on } \quad\left(r_{0},+\infty\right) \\
z\left(r_{0}\right)=z_{0}
\end{array}\right.
$$

is oscillatory.

Proof. First, we claim that the two conditions in (5.17) imply that

$$
A(r) v(r) \notin L^{1}(+\infty) .
$$

Indeed, from (3.23) and the second condition of (5.17) it follows that $\sqrt{A(r)} \notin$ $L^{1}(+\infty)$, and from Cauchy-Schwarz inequality

$$
\left(\int_{R}^{r} A(s) v(s) \mathrm{d} s\right)\left(\int_{R}^{r} \frac{\mathrm{d} s}{v(s)}\right) \geq\left(\int_{R}^{r} \sqrt{A(s)} \mathrm{d} s\right)^{2},
$$

we prove the claim by letting $r \rightarrow+\infty$.

Next suppose, by contradiction, that $z(r)$ has constant sign on $[\varrho,+\infty)$, for some $\varrho \geq r_{0}$. We define $y$ as in (5.5). Then $y \in \operatorname{Lip}_{\text {loc }}([\varrho,+\infty))$ and satisfies

$$
y^{\prime}=A v+\frac{y^{2}}{v}, \quad y(\varrho)=-\frac{v(\varrho) z^{\prime}(\varrho)}{z(\varrho)} \in \mathbb{R} .
$$

From $A \geq 0, y$ is non-decreasing. Integrating we get

$$
y(r) \geq y(R) \geq y(\varrho)+\int_{\varrho}^{R} A(s) v(s) \mathrm{d} s \quad \forall r>R>\varrho
$$

In both cases considered in the theorem the non integrability of $A(r) v(r)$ ensures the existence of $R>\varrho$ such that

$$
y(\varrho)+\int_{\varrho}^{R} A(s) v(s) \mathrm{d} s>0,
$$

therefore $y>0$ on $[R,+\infty)$. Now, we argue as in Theorem 5.1. In particular, integrating $(5.10)$ on $[r, \widetilde{R}]$ we get

$$
\frac{1}{y(r)} \geq \frac{1}{y(r)}-\frac{1}{y(\widetilde{R})} \geq \int_{r}^{\widetilde{R}} \frac{\mathrm{d} s}{v(s)} \geq \int_{r}^{\widetilde{R}} \frac{\mathrm{d} s}{f(s)} \quad \forall \widetilde{R}>r>R
$$

so that $1 / f \in L^{1}(+\infty)$, which contradicts (5.16). As for (5.17), from $y^{\prime} \geq 2 y \sqrt{A}$ a.e. on $[R,+\infty)$ we deduce

$$
y(r) \geq y(R) \exp \left\{2 \int_{R}^{r} \sqrt{A(s)} \mathrm{d} s\right\} \quad \forall r>R .
$$

Combining (5.19) and (5.21) with (5.20), letting $\widetilde{R} \rightarrow+\infty$ and using the definition of $\chi_{f}(r)$ we obtain

$\int_{R}^{r}\left(\sqrt{A(s)}-\sqrt{\chi_{f}(s)}\right) \mathrm{d} s \leq-\frac{1}{2}\left[\log \left(y(\varrho)+\int_{\varrho}^{R} A(s) v(s) \mathrm{d} s\right)+\log \int_{R}^{+\infty} \frac{\mathrm{d} s}{f(s)}\right]$

To complete the proof we let $r \rightarrow+\infty$ along a sequence realizing (5.17) to reach the desired contradiction. 
Remark 5.7. Condition (5.16) is due to W. Leighton, [Lei50]. The version in [Swa68], Theorem 2.24, does not assume $A \geq 0$, but the author substitutes the second requirement in (5.16) with the existence of

$$
\lim _{r \rightarrow+\infty} \int_{\varrho}^{r} A(s) v(s) \mathrm{d} s=+\infty,
$$

for some $\rho \in \mathbb{R}^{+}$. The argument is as follows. Assume by contradiction that $z$ has constant sign on $[\varrho,+\infty)$, and define $y$ as in (5.5). Integration of $y^{\prime} \geq A v$ with the aid of (5.23) gives the existence of $R>\varrho$ such that $y>0$ on $[R,+\infty)$. By (5.5) it follows that, if $z>0(\operatorname{resp} z<0)$ on $[R,+\infty), z^{\prime}<0\left(\operatorname{resp} z^{\prime}>0\right)$ on $[R,+\infty)$, thus $z(+\infty)$ exists and is finite. Let $z_{2}$ be the other linearly independent, positive solution of $\left(v z^{\prime}\right)^{\prime}+A v z=0$ on $(R,+\infty)$ given in Remark 3.9:

$$
z_{2}(r)=z(r) \int_{R}^{r} \frac{\mathrm{d} s}{v(s) z^{2}(s)} .
$$

Repeating the above argument for $z_{2}$ we deduce that $z_{2}(+\infty)$ exists and is finite. Letting $r \rightarrow+\infty$ in (5.24) and using $1 / v \notin L^{1}(+\infty)$ we reach the desired contradiction.

REMARK 5.8. By (3.22), (5.17) is equivalent to either one of the following requirements:

$$
\begin{aligned}
& \text { (i) } \quad \limsup _{r \rightarrow+\infty}\left(\int_{R}^{r} \sqrt{A(s)} \mathrm{d} s+\frac{1}{2} \log \int_{r}^{+\infty} \frac{\mathrm{d} s}{f(s)}\right)=+\infty ; \\
& \text { (ii) } \limsup _{r \rightarrow+\infty}\left(\int_{R}^{r} \sqrt{A(s)} \mathrm{d} s+\frac{1}{2} \log \int_{r}^{+\infty} \frac{\mathrm{d} s}{\widetilde{f}(s)}\right)=+\infty,
\end{aligned}
$$

where $\tilde{f} \sim C f$ as $r \rightarrow+\infty$, for some constant $C>0$.

Here are some stronger conditions which imply oscillation, and that will be used in the sequel.

Proposition 5.9. In the hypotheses of Theorem 5.6 on some interval $\left[r_{0},+\infty\right)$, and assuming also $1 / f \in L^{1}(+\infty)$, equation (5.18) is oscillatory if, for some $R \geq$ $r_{0}$, one of the following conditions is satisfied:

(i) $\quad A(r) \geq \chi_{f}(r) \quad$ on $[R,+\infty)$ and $\sqrt{A(r)}-\sqrt{\chi_{f}(r)} \notin L^{1}(+\infty)$;

(ii) $\limsup _{r \rightarrow+\infty} \frac{\int_{R}^{r} \sqrt{A(s)} \mathrm{d} s}{\int_{R}^{r} \sqrt{\chi_{f}(s)} \mathrm{d} s}>1$;

(iii) $\liminf _{r \rightarrow+\infty} \frac{\sqrt{A(r)}}{\sqrt{\chi_{f}(r)}}>1$;

(iv) $\limsup _{r \rightarrow+\infty} \frac{\int_{R}^{r} \sqrt{A(s)} \mathrm{d} s}{-\frac{1}{2} \log \int_{r}^{+\infty} \frac{\mathrm{d} s}{f(s)}}>1$;

(v) $\quad v \notin L^{1}(+\infty), A$ is non-decreasing and, for some sequence $\left\{r_{n}\right\} \uparrow+\infty$,

$$
\sqrt{A\left(r_{n}\right)}>\inf _{r>r_{n}}\left\{-\frac{1}{2} \frac{\log \int_{r}^{+\infty} \frac{\mathrm{d} s}{f(s)}}{r-r_{n}}\right\} .
$$


Proof. Implications $(i),(i i),(i i i),(i v)$ are immediate from (3.22) and (3.23). Regarding $(v)$, we proceed, by contradiction, as in Theorem 5.6, restricting the problem on $[\varrho,+\infty), \varrho>R_{0}$. Since $A(r)$ is non-decreasing and $v(r) \notin L^{1}(+\infty)$, we can choose $R>\varrho$ such that

$$
y(\varrho)+\int_{\varrho}^{R} A(s) v(s) \mathrm{d} s \geq 1 .
$$

Using the monotonicity of $A, v \leq f$ and the definition of $\chi_{f},(5.22)$ becomes

$$
\sqrt{A(R)}(r-R) \leq \int_{R}^{r} \sqrt{A(s)} \mathrm{d} s \leq-\frac{1}{2} \log \int_{r}^{+\infty} \frac{\mathrm{d} s}{v(s)} \leq-\frac{1}{2} \log \int_{r}^{+\infty} \frac{\mathrm{d} s}{f(s)}
$$

for every $R<r$; $(v)$ contradicts this last chain of inequalities.

Condition (5.17) in Theorem 5.6 exhibits clear analogies with Calabi condition (2.16) for the compactness of a complete Riemannian manifold $M$ with non-negative Ricci curvature. Indeed, this latter can be quite easily deduced from (5.17). Towards this aim, consider the problem

$$
\left\{\begin{array}{l}
g^{\prime \prime}+K(s) g=0 \\
g(0)=0, \quad g^{\prime}(0)=1,
\end{array}\right.
$$

with

$$
K(s)=K_{\gamma}(s)=\frac{\operatorname{Ricc}\left(\gamma^{\prime}, \gamma^{\prime}\right)(s)}{m-1} .
$$

Here $\gamma$ is a unit speed geodesic on the complete Riemannian manifold $M$ issuing from some reference origin $o$. As already observed in the proof of Theorem 2.1, $M$ is compact with finite fundamental group provided we can prove the existence of a first zero of $g$ for each $\gamma$.

Theorem 5.10 (Calabi criterion, [Cal67], Theorem 1). Let $(M,\langle\rangle$,$) be a com-$ plete Riemannian manifold of dimension $m \geq 2$ such that

$$
\text { Ricc } \geq 0 \quad \text { outside some compact set } C \text {. }
$$

Suppose that there exists an origin o for which, along every unit speed geodesic $\gamma$ issuing from o, we have

$$
\limsup _{s \rightarrow+\infty}\left(\int_{S}^{s} \sqrt{K_{\gamma}(\sigma)} \mathrm{d} \sigma-\frac{1}{2} \log s\right)=+\infty
$$

for some $S>0$ such that $C \subset B_{S}(o)$, and with $K_{\gamma}$ defined as in (5.27). Then, $M$ is compact and has finite fundamental group.

Proof. We prove that, in our assumptions, $g$ of (5.26) oscillates. Indeed, defining $r, z(r)$ as in Proposition 3.10, condition (5.28) is equivalent to the oscillatory condition

$$
\limsup _{r \rightarrow+\infty}\left(\int_{R}^{r} \sqrt{A(s)} \mathrm{d} s+\frac{1}{2} \log \int_{r}^{+\infty} \frac{\mathrm{d} s}{v(s)}\right)=+\infty
$$

of Remark 5.8 (with $v(r)=f(r)$ ) applied to the ODE (3.19), up to changing variables in the integrals according to (3.18). 


\subsection{Comparison with known criteria}

In the previous section we have observed that (5.17) is substantially equivalent to Calabi criterion for the oscillation of

$$
\frac{\mathrm{d}^{2} g}{\mathrm{~d} s^{2}}+K(s) g=0,
$$

once the substitution (3.18) is performed. In the light of the link between (5.18) and

$$
\ddot{\beta}+\left(\frac{A(r(t))}{\chi(r(t))}-1\right) \beta=0,
$$

obtained via the change of variables (4.6) with

$$
\beta(t)=e^{t} z(r(t)),
$$

we can compare (5.17) and (5.28) with some classical oscillation criteria for (5.29). Observe that $\beta$ oscillates if and only if so does $z$. Changing variables as in (4.6) and using (4.8), we rewrite (5.17) as the following condition for the oscillation of (5.29):

$$
\limsup _{t \rightarrow+\infty} \int_{T}^{t}\left(\sqrt{\frac{A(r(\sigma))}{\chi(r(\sigma))}}-1\right) \mathrm{d} \sigma=+\infty .
$$

On the other hand, a direct application of Calabi condition (5.28) to (5.29) yields oscillation whenever

$$
\frac{A(r(t))}{\chi(r(t))}-1 \geq 0, \quad \text { that is, } \quad A(r) \geq \chi(r), \quad \text { at least for } r>>1,
$$

and

$$
\limsup _{t \rightarrow+\infty}\left[\int_{T}^{t}\left(\sqrt{\frac{A(r(\sigma))}{\chi(r(\sigma))}-1}\right) \mathrm{d} \sigma-\frac{1}{2} \log t\right]=+\infty .
$$

Condition (5.30) has the advantage, on (5.32), that $A \geq \chi$ is not required. Furthermore, the negative part of the integrand in (5.30) may even be non-integrable in a neighbourhood of $+\infty$. However, if $A \geq \chi,(5.32)$ is in general better than (5.30). This can be seen, for instance, in the case

$$
\frac{A(r(\sigma))}{\chi(r(\sigma))}=1+\frac{C}{4 \sigma^{2}} \quad \text { on }[T,+\infty), \text { where } C>1 .
$$

Again, since $A$ may lie below $\chi,(5.30)$ is not contained in Hille-Nehari Theorem 2.7, so that (4.24) in Example 4.9, (1) does not contain (5.17). However, since

$$
\sqrt{\frac{A(r(s))}{\chi(r(s))}}-1 \leq \frac{A(r(s))}{\chi(r(s))}-1 \quad(\text { resp. } \geq) \quad \text { if } \frac{A(r(s))}{\chi(r(s))} \geq 1 \quad(\text { resp } \leq),
$$

Hille-Nehari condition

$$
\liminf _{t \rightarrow+\infty} t \int_{t}^{+\infty}\left(\frac{A(r(s))}{\chi(r(s))}-1\right) \mathrm{d} s>\frac{1}{4}
$$

is sharper than (5.30) when $A \geq \chi$. To show that (5.17) is not fully contained in the previous results, we therefore need to compare it with the oscillation criteria 
for (5.29) that allow a changing sign potential, as in the Moore condition on the existence of

$$
\lim _{t \rightarrow+\infty} \int_{T}^{t} \sigma^{\lambda}\left(\frac{A(r(\sigma))}{\chi(r(\sigma))}-1\right) \mathrm{d} \sigma=+\infty .
$$

for some $\lambda \in(0,1)$ (see Theorem 2.9). However, it is not hard to construct a function $h:[T,+\infty) \rightarrow \mathbb{R}_{0}^{+}$satisfying

$$
\limsup _{t \rightarrow+\infty} \int_{T}^{t}(\sqrt{h(\sigma)}-1) \mathrm{d} \sigma=+\infty,
$$

but for which

$$
\lim _{t \rightarrow+\infty} \int_{T}^{t} \sigma^{\lambda}(h(\sigma)-1) \mathrm{d} \sigma
$$

does not exist. This is possible since $\sigma^{\lambda} \notin L^{1}(+\infty)$. Therefore, (5.30) may yield information even in some cases when Moore theorem is not applicable. Thus, the next proposition can be used as an independent oscillation test.

Proposition 5.11. Let $K \in L_{\mathrm{loc}}^{\infty}([T,+\infty))$, and consider the ODE

$$
g^{\prime \prime}+K(t) g=0 \text {. }
$$

Assume that $K \geq-B^{2}$, for some $B>0$. Then, the ODE is oscillatory provided

$$
\limsup _{t \rightarrow+\infty} \int_{T}^{t}(\sqrt{K(\sigma)+B}-B) \mathrm{d} \sigma=+\infty .
$$

Proof. The case $B=1$ reduces to (5.30) with

$$
K(t)=\frac{A(r(t))}{\chi(r(t))}-1
$$

Note that if we fix some critical function $\chi$, for instance, that of a polynomial volume growth, then by (4.6) $A$ is uniquely determined by $K$ and viceversa. This enables us to apply (5.30) directly to $g^{\prime \prime}+K g=0$. For general $B$, we reduce to the case $B=1$ by setting $\widetilde{g}(t)=g\left(B^{-1} t\right)$. Since $\widetilde{g}$ solves $\widetilde{g}^{\prime \prime}+B^{-2} K\left(B^{-1} t\right) \widetilde{g}=0$, we conclude by changing variable in (5.30).

REMARK 5.12. Expression (5.35) has the same structure as (5.28), and it will be generalized in Theorem 5.41 to the case of a non-constant negative lower bound for $K$.

We observe that (see also Proposition 3.10), the choices

$$
s(r)=\left(\int_{r}^{+\infty} \frac{\mathrm{d} s}{v(s)}\right)^{-1}, \quad g(s)=s z(r(s))
$$

and

$$
t(r)=-\frac{1}{2} \log \int_{r}^{+\infty} \frac{\mathrm{d} s}{v(s)}, \quad \beta(t)=e^{t} z(r(t))
$$

are different ways to produce an equation of the type $g^{\prime \prime}+K g=0$ from $\left(v z^{\prime}\right)^{\prime}+$ $A v z=0$. Furthermore, $z, g, \beta$ share the same oscillatory (or nonoscillatory) behaviour. Therefore, combining the two changes of variables we can pass from the ODE $\ddot{\beta}+F \beta=0$ to the ODE $g^{\prime \prime}+K g=0$ or viceversa according to which potential, $F$ or $G$, is easier to handle for the specific problem under consideration. In fact, it 
can be checked through (5.36) and (5.37) that, if $g$ solves $g^{\prime \prime}+K(s) g=0$ on some $[S,+\infty)$, then $\beta(t)=e^{-t} g\left(e^{2 t}\right)$ and it satisfy

$$
\ddot{\beta}(t)+\left(4 K\left(e^{2 t}\right) e^{4 t}-1\right) \beta(t)=0 .
$$

Viceversa, if $\beta$ solves $\ddot{\beta}+F \beta=0$, then $g(s)=\beta\left(\frac{1}{2} \log s\right)$ and it solves

$$
\frac{\mathrm{d}^{2} g}{\mathrm{~d} s^{2}}+\frac{K(t(s))+1}{4 s^{2}} g=0 .
$$

The above observation gives rise to the next

Proposition 5.13. Let $K \in L_{\mathrm{loc}}^{\infty}\left(\left[s_{0},+\infty\right)\right)$, for some $s_{0}>0$. Then, the equation $g^{\prime \prime}+K(s) g=0$ oscillates if and only if, for some (hence any) $B>0$, $a>0$, the same happens to one of the following ordinary differential equations:

$$
\begin{aligned}
& \text { (i) } \ddot{\beta}(t)+a^{2}\left(\frac{4 K\left(B^{-1} e^{2 a t}\right)}{B^{2}} e^{4 a t}-1\right) \beta(t)=0 ; \\
& \text { (ii) } \ddot{\beta}(t)+\frac{1}{4 B^{2} t^{2}}\left[B^{2}+K\left(\frac{\log t}{2 B}\right)\right] \beta(t)=0 .
\end{aligned}
$$

Proof. As for $(i)$, it is enough to set

$$
\beta(t)=e^{-a t} g\left(B^{-1} e^{2 a t}\right),
$$

while $(i i)$ is obtained by means of the change of variables

$$
\beta(t)=\sqrt{t} g\left(\frac{\log t}{2 B}\right) .
$$

Clearly, in both cases $g$ oscillates if and only if so does $\beta$.

It is worth to observe that case ( $i i)$ of Proposition 5.13 enables us to deal with an ODE with non-negative potential whenever $K$ is bounded from below. For instance, applying Hille-Nehari Theorem 2.7 to $(i i)$ and changing variables, we get the following simple criterion.

Corollary 5.14. Assume that $K(s) \geq-B^{2}$ on $\left[s_{0},+\infty\right)$, for some $B>0$. Then, a solution of $g^{\prime \prime}+K(s) g=0$ oscillates if

$$
\liminf _{s \rightarrow+\infty} e^{2 B s} \int_{s}^{+\infty} \frac{1}{e^{2 B \sigma}}\left(B^{2}+K(\sigma)\right) \mathrm{d} \sigma>\frac{B}{2},
$$

while it has eventually constant sign when

$$
e^{2 B s} \int_{s}^{+\infty} \frac{1}{e^{2 B \sigma}}\left(B^{2}+K(\sigma)\right) \mathrm{d} \sigma \leq \frac{B}{2} \quad \text { on }\left[s_{1},+\infty\right),
$$

for some $s_{1} \geq s_{0}$.

This is, roughly speaking, the "Hille-Nehari type" counterpart of Proposition 5.11. Clearly, compactness results for manifolds satisfying

$$
\operatorname{Ricc}(\nabla r, \nabla r) \geq-(m-1) B^{2}
$$

follow from Proposition 5.11 and Corollary 5.14. 


\subsection{Instability and index of $-\Delta-q(x)$}

Corollary 5.2 and Theorem 5.6 can be applied to yield upper bounds on the bottom of the spectrum of a Schrödinger operator $L=-\Delta-q(x)$. We let $v(r)=$ $\operatorname{vol}\left(\partial B_{r}\right)$, and we denote with $\bar{q}(r)$ the spherical mean of $q(x)$, that is,

$$
\bar{q}(r)=\frac{1}{\operatorname{vol}\left(\partial B_{r}\right)} \int_{\partial B_{r}} q \quad \in L_{\mathrm{loc}}^{\infty}\left(\mathbb{R}_{0}^{+}\right)
$$

Observe that, by the coarea formula,

$$
\int_{0}^{R} \bar{q}(s) v(s) \mathrm{d} s=\int_{0}^{R}\left(\int_{\partial B_{s}} q\right) \mathrm{d} s=\int_{B_{R}} q .
$$

Theorem 5.15. Let $(M,\langle\rangle$,$) be a complete Riemannian manifold of dimension$ $m \geq 2$, and let $q(x) \in L_{\mathrm{loc}}^{\infty}(M)$ be such that its spherical mean $\bar{q}(r)$ satisfies

$$
\bar{q}(r) \geq 0, \quad \bar{q}(r) \not \equiv 0 .
$$

Let $f(r)$ satisfy $(\mathrm{F} 1)$ with $v(r)=\operatorname{vol}\left(\partial B_{r}\right)$ on $\mathbb{R}^{+}$. Consider the following assumptions:

(i) either

$$
1 / f \notin L^{1}(+\infty)
$$

or $1 / f \in L^{1}(+\infty)$ and there exist $r>R$ such that $\bar{q}(r) \not \equiv 0$ on $[0, R]$ and

$$
\int_{R}^{r}\left(\sqrt{\bar{q}(s)}-\sqrt{\chi_{f}(s)}\right) \mathrm{d} s>-\frac{1}{2}\left(\log \int_{B_{R}} q+\log \int_{R}^{+\infty} \frac{\mathrm{d} s}{f(s)}\right) ;
$$

(ii) either

or

$$
1 / f \notin L^{1}(+\infty), \quad q(x) \notin L^{1}(M)
$$

$$
1 / f \in L^{1}(+\infty) \quad \text { and } \quad \limsup _{r \rightarrow+\infty} \int_{R}^{r}\left(\sqrt{\bar{q}(s)}-\sqrt{\chi_{f}(s)}\right) \mathrm{d} s=+\infty
$$

for some large $R$.

Then,

- under assumption ( $i)$ the Schrödinger operator $L=-\Delta-q(x)$ has negative spectral radius, that is,

$$
\lambda_{1}^{L}(M)<0
$$

- under assumption (ii) L has infinite index.

Furthermore, if

$$
f(r)=\Lambda r^{\theta} \exp \left\{a r^{\alpha} \log ^{\beta} r\right\}, \quad \text { for some } \Lambda, a, \alpha>0, \beta \geq 0, \theta \in \mathbb{R},
$$

(5.43) is equivalent to

$$
\limsup _{r \rightarrow+\infty}\left[\int_{R}^{r} \sqrt{\bar{q}(s)} \mathrm{d} s-\frac{a}{2} r^{\alpha} \log ^{\beta} r-\frac{\alpha+\theta-1}{2} \log r-\frac{\beta}{2} \log \log r\right]=+\infty
$$

while if $f(r)=\Lambda r^{\alpha}$, for some $\alpha>1$, then (5.43) is equivalent to

$$
\limsup _{r \rightarrow+\infty}\left[\int_{R}^{r} \sqrt{\bar{q}(s)} \mathrm{d} s-\frac{\alpha-1}{2} \log r\right]=+\infty .
$$


Proof. We follow the reasoning outlined in the Introduction. By Corollary 3.7, we choose a locally Lipschitz solution $z(r)$ of (5.2), with $A(r)=\bar{q}(r)$. According to Corollary 5.2, $z$ has a first zero at some $R$. We define $\psi(x)=z(r(x))$ if $x \in B_{R}$, $\psi(x)=0$ otherwise, so that $\psi \in \operatorname{Lip}_{0}\left(B_{R}\right)$. Using the coarea formula and Gauss lemma, and integrating by parts, we obtain

$$
\begin{aligned}
\int_{B_{R}}|\nabla \psi|^{2}-q(x) \psi^{2} & =\int_{B_{R}}|\nabla \psi|^{2}-\bar{q}(r) \psi^{2} \\
& =-\int_{0}^{R} z(r)\left[\left(v(r) z^{\prime}(r)\right)^{\prime}+\bar{q}(r) v(r) z(r)\right] \mathrm{d} r=0 .
\end{aligned}
$$

By the min-max characterization (1.78) and domain monotonicity we conclude $\lambda_{1}^{L}(M)<0$. The proof of $(i i)$ is similar. Let $\Omega$ be any relatively compact set of $M$, and let $R$ be sufficiently large that $\Omega \Subset B_{R}$. By Corollaries 3.7 and 5.6, a solution $z$ of (5.18) is oscillatory. Let $R_{1}, R_{2}$ be two consecutive zeroes after $R$, and define $\psi(x)=z(r(x))$ on $B_{R_{2}} \backslash B_{R_{1}}$, and zero otherwise. Then, as in (5.48) we get

$$
\int_{B_{R_{2}} \backslash \Omega}|\nabla \psi|^{2}-q(x) \psi^{2}=0
$$

and by domain monotonicity $\lambda_{1}^{L}(M \backslash \Omega)<0$. By Theorem 1.40, $\operatorname{ind}_{L}(M)=+\infty$. When $f(r)$ has the expression (5.45),

$$
\int_{r}^{+\infty} \frac{\mathrm{d} s}{f(s)} \sim \frac{1}{\Lambda}\left(\frac{1}{a \alpha}\right) r^{1-\alpha-\theta} \log ^{-\beta} r \exp \left\{-a r^{\alpha} \log ^{\beta} r\right\},
$$

and we conclude using Remark 5.8 to get (5.46). The case of a polynomial $f$ is analogous.

As an immediate application of the above result, we state a particular version of Theorem 2.30. It seems to us that this is the first instance of an existence result for Yamabe-type equations that does not require pointwise bounds on either the sectional or the Ricci curvatures.

THEOREM 5.16. Let $(M,\langle\rangle$,$) be a non-compact Riemannian manifold of di-$ mension $m \geq 2$, and let $q(x), b(x) \in C_{\mathrm{loc}}^{0, \mu}(M), \mu \in(0,1]$. Suppose that $b(x)>0$ on $M$. Denote with $\bar{q}(r)$ the spherical mean of $q$, and assume

$$
\bar{q} \geq 0 \quad \text { on } \mathbb{R}^{+}, \quad \bar{q} \not \equiv 0 .
$$

Let $f$ satisfy $(\mathrm{F} 1)$ with $v(r)=\operatorname{vol}\left(\partial B_{r}\right)$ on $\mathbb{R}^{+}$. If either

$$
1 / f \notin L^{1}(+\infty)
$$

or $1 / f \in L^{1}(+\infty)$ and there exist $r>R$ such that $\bar{q} \not \equiv 0$ on $[0, R]$ and

$$
\int_{R}^{r}\left(\sqrt{\bar{q}(s)}-\sqrt{\chi_{f}(s)}\right) \mathrm{d} s>-\frac{1}{2}\left(\log \int_{B_{R}} q+\log \int_{R}^{+\infty} \frac{\mathrm{d} s}{f(s)}\right),
$$

then, for every $\sigma>1$, the equation

$$
\Delta u+q(x) u-b(x) u^{\sigma}=0
$$

possesses a minimal and a maximal (possibly coinciding) positive $C_{\mathrm{loc}}^{2, \mu}$ solutions. 
On the links between $\chi$ and $\tilde{\chi}$, III. This last observation is related to those at the end of Sections 4.2 and 4.5. Indeed, case (ii) of Theorem 5.15 can be used to show that, in many instances, the function $\tilde{\chi}$ is not an adequate critical function. Towards this purpose, we suppose that $\left(M_{g}, \mathrm{~d} s^{2}\right)$ is a model manifold, and we set $f(r)=v(r)=g(r)^{m-1}$. Furthermore, we assume that $g$ is non-decreasing and $g^{1-m} \in L^{1}(+\infty)$. Let $q(x) \in L_{\text {loc }}^{\infty}(M)$ be such that $0<q \leq \tilde{\chi}$ on $M$ and $q=\tilde{\chi}$ on $M \backslash B_{1}$, and define, as usual, $L=-\Delta-q(x)$. For $R \geq 1$, by (4.55) we deduce

$\int_{R}^{r}(\sqrt{\bar{q}(s)}-\sqrt{\chi(s)}) \mathrm{d} s=-\frac{1}{2} \int_{R}^{r}(\log (2 \sqrt{\chi(r)}))^{\prime} \mathrm{d} s=\frac{1}{2} \log \left(\frac{1}{2 \sqrt{\chi(r)}}\right)+O(1)$

as $r \rightarrow+\infty$. If the critical curve satisfies the property

$$
\liminf _{r \rightarrow+\infty} \chi(r)=0
$$

then, by $(i i)$ of Theorem 5.15 ,

$$
\operatorname{ind}_{L}(M)=+\infty \text {. }
$$

As a consequence, whenever (5.53) is met, the uncertainty principle cannot hold with $\chi$ replaced by $\tilde{\chi}$, for otherwise (by our definition of $q(x)$ ) the operator $L$ should have non-negative spectral radius on $M$, contradicting (5.54). By Corollary 3.25, condition (5.53) is satisfied if, for instance,

$$
\operatorname{Ricc}(\nabla r, \nabla r)(x) \geq-(m-1) G(r),
$$

for some non-negative $G \in C^{0}\left(\mathbb{R}_{0}^{+}\right)$such that $G(r) \rightarrow 0$ as $r \rightarrow+\infty$. On the other hand, it is easy to construct examples when $g(r)$ has faster than exponential growth and $\chi$ is better than $\tilde{\chi}$. For instance, if

$$
g(r)=\frac{\exp \left\{a r^{\alpha}\right\}}{r^{\alpha-1}} \quad \text { on }\left[r_{0},+\infty\right), \quad \text { where } \quad a>0, \alpha>1,
$$

then $\chi(r)=a^{2} \alpha^{2} / 4 r^{2(\alpha-1)}$ is increasing on $\left[r_{0},+\infty\right)$, thus, by (4.57), $\chi>\tilde{\chi}$ on $\left[r_{0},+\infty\right)$, as can be seen also by a direct computation. The case of exponential growth reveals to be the most subtle. In fact, it may also present an unpleasant feature that we describe for the prototype example of $\mathbb{H}_{B}^{3}$, the hyperbolic 3 -space of sectional curvature $-B^{2}<0$. As observed at the end of Section 4.2, in [BR97] the authors proved that $\tilde{\chi}$ is, indeed, a critical function on each manifold of dimension $m \geq 3$ satisfying $K_{\mathrm{rad}} \leq-B^{2}$. Since by (3.31) $\chi$ is decreasing on $\mathbb{H}_{B}^{3}$, applying (4.57) we conclude that $\tilde{\chi}>\chi$ on $\mathbb{R}^{+}$, that is, $\tilde{\chi}$ is better than $\chi$ as a critical curve on $\mathbb{H}_{B}^{3}$. This particular case motivates the following

\section{Questions:}

(1) Which is the optimal uncertainty principle on the hyperbolic space $\mathbb{H}_{B}^{m}$ or, more generally, on manifolds satisfying $K_{\mathrm{rad}} \leq-B^{2}$ ?

(2) Why, in this setting, $\widetilde{\chi}$ may be better than $\chi$ ?

\subsection{Some remarks on minimal surfaces}

The aim of this section is to present a typical situation where the case $1 / f \notin$ $L^{1}(+\infty)$ in Theorem 5.15 occurs. Such example concerns minimal surfaces with 
finite stability index in some ambient 3-manifold. To begin with, and to fix notations, we recall some preliminary facts. Suppose we are given an isometrically immersed hypersurface

$$
\varphi: M^{m} \longrightarrow N^{m+1}
$$

where $N$ is orientable. We fix the index notation $i, j, k, t \in\{1, \ldots, m\}$, and we choose a local Darboux frame $\left\{e_{i}, \nu\right\}$. Let $R$, Ricc, $s$ (resp $\bar{R}, \overline{\text { Ricc }}, \bar{s}$ ) be the curvature tensor, the Ricci tensor and the scalar curvature of $M$ (resp. $N$ ), denote with $I I=\left(h_{i j}\right)$ the second fundamental form of the immersion in the direction of $\nu$, with $|I I|^{2}$ the square of its norm and with $H=m^{-1} h_{i i} \nu$ the mean curvature vector. Tracing twice the Gauss equations

$$
R_{i j k t}=\bar{R}_{i j k t}+h_{i k} h_{j t}-h_{i t} h_{j k}
$$

we get

$$
s=\bar{s}-2 \overline{\operatorname{Ricc}}(\nu, \nu)+m^{2} H^{2}-|I I|^{2} .
$$

A minimal immersion $\varphi$ is characterized by $H \equiv 0$, which is equivalent to say that $\varphi$ is a stationary point for the volume functional on every relatively compact domain with smooth boundary in $M$. If we restrict to those variations of the volume functional that are driven by functions $f \in C_{c}^{\infty}(M)$ satisfying

$$
\int_{M} f=0
$$

then it can be proved that the stationary points are characterized to be the constant mean curvature (shortly, CMC) hypersurfaces. In both the minimal and the CMC cases, we say that $\varphi$ is stable if it locally minimizes the volume functional up to second order, and unstable otherwise. Analytically the condition of stability is expressed by

$$
\int_{M}|\nabla \psi|^{2}-\left(|I I|^{2}+\overline{\operatorname{Ricc}}(\nu, \nu)\right) \psi^{2} \geq 0 \quad \forall \psi \in C_{c}^{\infty}(M),
$$

that is,

$$
\lambda_{1}^{L}(M) \geq 0, \quad \text { where } \quad L=-\Delta-\left(|I I|^{2}+\overline{\operatorname{Ricc}}(\nu, \nu)\right) .
$$

Following S.T. Yau and R. Schoen [YS79], the potential in $L$ can be rearranged to make the scalar curvatures appear. Indeed, by (5.57)

$$
\begin{aligned}
\overline{\operatorname{Ricc}}(\nu, \nu)+|I I|^{2} & =\frac{1}{2}\left(\bar{s}-s+m^{2} H^{2}-|I I|^{2}\right)+|I I|^{2} \\
& =\frac{1}{2}\left(\bar{s}-s+m^{2} H^{2}+|I I|^{2}\right) .
\end{aligned}
$$

In particular, if $M$ is a CMC surface with Gaussian curvature $K$, this gives the following expression for the stability operator:

$$
L=-\Delta-\left(\overline{\operatorname{Ricc}}(\nu, \nu)+|I I|^{2}\right)=-\Delta-\frac{1}{2}\left(4 H^{2}+\bar{s}+|I I|^{2}\right)+K .
$$

Next, we recall that a surface $M$ is of finite topological type (or, equivalently, finitely connected) if it is homeomorphic to a compact surface $\Sigma$ with finitely many points $\left\{p_{1}, \ldots, p_{h}\right\}$ removed. In this case, around each $p_{i}$ we can choose a small 
open disk $D_{i}$ in $\Sigma$ such that the $D_{i}$ are pairwise disjoint and $M$ is homeomorphic to $\Sigma \backslash\left(\bigcup_{i} \bar{D}_{i}\right)$. Then, we can define the Euler characteristic of $M$ as

$$
\chi_{E}(M)=\chi_{E}\left(\Sigma \backslash\left(\bigcup_{i} D_{i}\right)\right)=\chi_{E}(\Sigma)-h .
$$

The stability operator in (5.60) has the general form $L=-\Delta-V+a K$, where $V \in$ $L_{\mathrm{loc}}^{\infty}(M)$ and $a>0$ is a constant. In the case $V \equiv 0$, the operator $L_{a}=-\Delta+a K$ has been investigated by D. Fischer-Colbrie and R. Schoen in connection with the type problem for a Riemann surface. In the celebrated paper [FCS80], they pose some questions about topological restrictions deriving from spectral assumptions. With the aid of a powerful integral inequality due to T. Colding and W. Minicozzi [CM02] (which has its germ in the work of A.V. Pogorelov [Pog81]), P. Castillon [Cas06] and later J.M. Espinar and H. Rosenberg [ER11] succeeded in solving most of the problems in [FCS80]. Colding-Minicozzi method has been independently developed by W. Meeks, J. Perez and A. Ros in [MPR08]. Starting from the estimates in [Cas06], [MPR08], and combining with Theorem 5.15, we shall now recover some well-known interesting results in the literature.

We begin with a topological Lemma.

Lemma 5.17 ([Cas06], Lemma 2.4). Let $(M,\langle\rangle$,$) be a complete Riemannian$ surface, and let $\left\{\Omega_{j}\right\}$ be any exhaustion of $M$.

(i) If $M$ is of finite topological type, there exists $j_{0}$ such that, for every $j \geq j_{0}$, $\chi_{E}\left(\Omega_{j}\right) \leq \chi_{E}(M)$;

(ii) If $M$ is not of finite topological type, $\lim _{j} \chi_{E}\left(\Omega_{j}\right)=-\infty$.

Next, we describe the estimate in [Cas06] and [MPR08]. A partial and less powerful result with the same method has appeared in our recent work [BMR09], when we still did not know about the papers of P. Castillon, W. Meeks, J. Perez and A. Ros. We apologize to these authors for the omitted citation.

Set

$$
l(r)=\operatorname{vol}\left(\partial B_{r}\right), \quad k(r)=\int_{B_{r}} K .
$$

Proposition 5.18 ([Cas06], Propositions 3.1 and 3.2, and [MPR08]). Let $(M,\langle\rangle$,$) be a complete Riemann surface, and let V \in L_{\mathrm{loc}}^{\infty}(M)$ be such that $V_{-} \in$ $L^{1}(M)$. Fix a constant $a>1 / 4$, and suppose that the operator $L=-\Delta-V+a K$ has finite index. Then, $M$ is of finite topological type,

$$
V \in L^{1}(M) \quad \text { and } \quad \operatorname{vol}\left(B_{r}\right) \leq C r^{2},
$$

for some $C>0$ and for $r \in \mathbb{R}^{+}$.

Proof. By Theorem 1.40, let $R_{0}$ be sufficiently large that $\lambda_{1}^{L}\left(M \backslash B_{R_{0}-1}\right) \geq 0$. We choose $R>R_{0}+1$ and we consider a function

$$
\psi_{R} \in \operatorname{Lip}\left(B_{R} \backslash B_{R_{0}}\right) \quad \text { such that } \begin{cases}\psi_{R} \equiv 0 & \text { on } \partial B_{R_{0}}, \\ \psi_{R} \equiv 1 & \text { on } \partial B_{R} .\end{cases}
$$

Denote with $c_{a, R}$ the constant

$$
c_{a, R}=\int_{B_{R} \backslash B_{R_{0}}}\left[\left|\nabla \psi_{R}\right|^{2}+a K \psi_{R}^{2}-V \psi_{R}^{2}\right]
$$


Let now $r>R$ and let $f_{r}:[R, r] \rightarrow[0,1]$ be a $C^{2}$ function such that

$$
f_{r}(R)=1, \quad f_{r}(r)=0, \quad f_{r}^{\prime} \leq 0, \quad f_{r}^{\prime \prime} \geq 0,
$$

and set $\psi_{r}(x)=f_{r}(r(x))$. Then, by the coarea formula and integrating twice by parts

$$
\begin{aligned}
\int_{B_{r} \backslash B_{R}} a K \psi_{r}^{2} & =a \int_{R}^{r} f_{r}^{2}(t)\left[\int_{\partial B_{t}} K\right] \mathrm{d} t=-a \int_{R}^{r}\left(f_{r}^{2}(t)\right)^{\prime} k(t) \mathrm{d} t-a k(R) \\
& =a \int_{R}^{r}\left(f_{r}^{2}(t)\right)^{\prime \prime}\left[\int_{R}^{t} k\right] \mathrm{d} t-a k(R) .
\end{aligned}
$$

Now, from (1.21)

$$
\int_{R}^{t} k(s) \mathrm{d} s \leq 2 \pi \int_{R}^{t} \chi_{E}(s) \mathrm{d} s-l(t)+l(R)
$$

and since $\left(f_{r}^{2}\right)^{\prime \prime} \geq 0$, the RHS of (5.64) is bounded above by

$$
2 \pi a \int_{R}^{r}\left(f_{r}^{2}(t)\right)^{\prime \prime}\left[\int_{R}^{t} \chi_{E}\right] \mathrm{d} t-a \int_{R}^{r}\left(f_{r}^{2}(t)\right)^{\prime \prime} l(t) \mathrm{d} t-a k(R)-2 a f_{r}^{\prime}(R) l(R) .
$$

Therefore, setting

$$
\psi_{r}(x)= \begin{cases}\psi_{R}(x) & \text { if } r(x) \in\left[R_{0}, R\right] \\ f_{r}(r(x)) & \text { if } r(x) \in[R, r] \\ 0 & \text { otherwise, }\end{cases}
$$

combining (5.62), (5.64), (5.65) and $\lambda_{1}^{L}\left(M \backslash B_{R_{0}-1}\right) \geq 0$ we get

$$
\begin{aligned}
0 \leq & \int_{M}\left[\left|\nabla \psi_{r}\right|^{2}+a K \psi_{r}^{2}-V \psi_{r}^{2}\right] \\
\leq & \int_{R}^{r}\left(\left(f_{r}^{\prime}(t)\right)^{2}-a\left(f_{r}^{2}(t)\right)^{\prime \prime}\right) l(t) \mathrm{d} t+2 \pi a \int_{R}^{r}\left(f_{r}^{2}(t)\right)^{\prime \prime}\left[\int_{R}^{t} \chi_{E}\right] \mathrm{d} t+ \\
& +\left[c_{a, R}-a k(R)-2 a f_{r}^{\prime}(R) l(R)\right]-\int_{M} V \psi_{r}^{2} .
\end{aligned}
$$

Now, the Euler characteristic of the compact surface with boundary, $\bar{B}_{r}$, is bounded above by 1 . We can thus set

$$
E=E(R)=\sup _{s \in[R,+\infty)} \chi_{E}(s), \quad E(R) \in(-\infty, 1] .
$$

Again, since $\left(f_{r}^{2}\right)^{\prime \prime} \geq 0$, integrating by parts we obtain

$$
2 \pi a \int_{R}^{r}\left(f_{r}^{2}(t)\right)^{\prime \prime}\left[\int_{R}^{t} \chi_{E}\right] \mathrm{d} t \leq 2 \pi a E \int_{R}^{r}\left(f_{r}^{2}(t)\right)^{\prime \prime}(t-R) \mathrm{d} t=2 \pi a E,
$$


so that

$$
\begin{aligned}
0 \leq & \int_{M}\left[\left|\nabla \psi_{r}\right|^{2}+a K \psi_{r}^{2}-V \psi_{r}^{2}\right] \\
\leq & \int_{R}^{r}\left(\left(f_{r}^{\prime}(t)\right)^{2}-a\left(f_{r}^{2}(t)\right)^{\prime \prime}\right) l(t) \mathrm{d} t+ \\
& +\left[c_{a, R}-a k(R)-2 a f_{r}^{\prime}(R) l(R)+2 \pi a E\right]-\int_{M} V \psi_{r}^{2} .
\end{aligned}
$$

Choose

$$
f_{r}(t)=\left(\frac{r-t}{r-R}\right)^{\beta}
$$

where $\beta \geq 1$ has to be specified later, and note that (5.63) are met. A straightforward computation gives $f_{r}^{\prime}(R) \rightarrow 0$ as $r \rightarrow+\infty$ and

$$
\int_{R}^{r}\left(\left(f_{r}^{\prime}(t)\right)^{2}-a\left(f_{r}^{2}(t)\right)^{\prime \prime}\right) l(t) \mathrm{d} t=\frac{\beta^{2}(1-4 a)+2 a \beta}{(r-R)^{2 \beta}} \int_{R}^{r}(r-t)^{2 \beta-2} l(t) \mathrm{d} t .
$$

Since $a>1 / 4$, the constant

$$
c_{\beta}=-\left(\beta^{2}(1-4 a)+2 a \beta\right)
$$

can be made as big as we wish, up to choosing $\beta$ big enough. In particular, the RHS of the above equality is negative provided $c_{\beta}>0$. If we assume $r>2 R$, from

$$
\int_{R}^{r}(r-t)^{2 \beta-2} l(t) \mathrm{d} t \geq \int_{R}^{r / 2}(r-t)^{2 \beta-2} l(t) \mathrm{d} t \geq\left(\frac{r}{2}\right)^{2 \beta-2} \operatorname{vol}\left(B_{r / 2} \backslash B_{R}\right),
$$

inserting into (5.69) we obtain

$$
\begin{aligned}
\int_{R}^{r}\left(\left(f_{r}^{\prime}(t)\right)^{2}-a\left(f_{r}^{2}(t)\right)^{\prime \prime}\right) l(t) \mathrm{d} t & \leq-\frac{c_{\beta}}{2^{2 \beta-2}} \frac{r^{2 \beta-2}}{(r-R)^{2 \beta}} \operatorname{vol}\left(B_{r / 2} \backslash B_{R}\right) . \\
& \leq-\widetilde{c}_{\beta} \frac{\operatorname{vol}\left(B_{r / 2} \backslash B_{R}\right)}{(r / 2-R)^{2}}
\end{aligned}
$$

for some $\widetilde{c}_{\beta}$ only depending on $\beta$. Thus, from (5.68) we deduce the following estimate:

$$
\widetilde{c}_{\beta} \frac{\operatorname{vol}\left(B_{r / 2} \backslash B_{R}\right)}{(r / 2-R)^{2}}+\int_{B_{r} \backslash B_{R}} V \psi_{r}^{2} \leq c_{a, R}+a(2 \pi E-k(R))+o(1) .
$$

as $r \rightarrow+\infty$. Since $V_{-} \in L^{1}(M)$ and $\psi_{r} \uparrow 1$ pointwise on $M \backslash B_{R}$, we get from (5.70)

$$
\lim _{r \rightarrow+\infty} \int_{B_{r} \backslash B_{R}} V_{+} \psi_{r}^{2} \leq \limsup _{r \rightarrow+\infty} \int_{B_{r} \backslash B_{R}} V \psi_{r}^{2}+\lim _{r \rightarrow+\infty} \int_{B_{r} \backslash B_{R}} V_{-} \psi_{r}^{2}<+\infty,
$$

hence $V \in L^{1}(M)$ by the monotone convergence theorem. The bound $\operatorname{vol}\left(B_{r}\right) \leq$ $C r^{2}$ for $r>>1$ is immediate from (5.70), and by the asymptotic (1.63) the same estimate holds near $r=0$ up to changing $C$. To prove that $M$ is of finite topological type, we consider

$$
\psi_{R}(x)= \begin{cases}r(x)-R_{0} & \text { if } r(x) \in\left[R_{0}, R_{0}+1\right), \\ 1 & \text { if } r(x) \in\left[R_{0}+1, R\right],\end{cases}
$$


so that

$$
\begin{aligned}
c_{a, R}= & \left(\int_{B_{R_{0}+1} \backslash B_{R_{0}}}\left[\left|\nabla \psi_{R}\right|^{2}+a K \psi_{R}^{2}\right]+\int_{B_{R} \backslash B_{R_{0}}} V \psi_{R}^{2}\right) \\
& +a k(R)-a K\left(R_{0}+1\right) \\
\leq & \widehat{C}+a k(R),
\end{aligned}
$$

where $\widehat{C}$ is a constant depending on $a$, on the geometry of $M$ on $B_{R_{0}+1}$ and on the $L^{1}$ norm of $V$ on $M \backslash B_{R_{0}}$. Inserting into (5.70) and letting $r \rightarrow+\infty$ we deduce that

$$
\widehat{C}+2 \pi a E \geq \int_{M \backslash B_{R}} V .
$$

Hence, $E$ cannot diverge as $R \rightarrow+\infty$. By definition (5.67) and Lemma 5.17, $M$ is of finite topological type.

REMARK 5.19. We note in passing that condition $\operatorname{vol}\left(B_{r}\right)=O\left(r^{2}\right)$ implies the parabolicity of the surface, according to a result in [CY75] (see also Theorem 5.1 of [PRS05], together with Lemma 5.22 below). Hence, each end $E$ with respect to some compact set $K$ is conformally parabolic. Since $M$ is of finite topological type, if $K$ is sufficiently large then $E$ is a cylinder, so that $E$ must be conformally diffeomorphic to the punctured disk $\left(D \backslash\{0\},|\mathrm{d} z|^{2}\right) \subset \mathbb{C}$, as shown in ([Cas06], Proposition 3.3).

Remark 5.20. A posteriori, since $M$ is of finite topological type, by Lemma 5.17 the constant $E$ can be chosen to be $\chi(M)$. Furthermore, in inequality (5.70), only $c_{a, R}$ depends on the choice of $\psi_{R}$ in (5.61). If we vary $\psi_{R}$ among the class $\mathcal{A}$ of Lipschitz functions that are zero on $\partial B_{R_{0}}$ and 1 on $\partial B_{R}$, the best value of $c_{a, R}$ is realized by the $L$-capacity

$$
\operatorname{cap}_{L}\left(B_{R} \backslash B_{R_{0}}\right)=\inf _{\phi \in \mathcal{A}} \int_{B_{R} \backslash B_{R_{0}}}\left(|\nabla \phi|^{2}+a K \phi^{2}-V \phi^{2}\right) .
$$

Clearly, $\operatorname{cap}_{L}\left(B_{R} \backslash B_{R_{0}}\right)$ is non-increasing as a function of $R$, and we can define

$$
\operatorname{cap}_{L}\left(M \backslash B_{R_{0}}\right)=\lim _{R \rightarrow+\infty} \operatorname{cap}_{L}\left(B_{R} \backslash B_{R_{0}}\right) .
$$

Consequently, if we set $v^{*}=\lim \sup _{r \rightarrow+\infty} \operatorname{vol}\left(B_{r}\right) / r^{2}$, letting first $r \rightarrow+\infty$ and then $R \rightarrow+\infty$ along a suitable sequence we deduce

$$
\widetilde{c}_{\beta} v^{*} \leq \operatorname{cap}_{L}\left(M \backslash B_{R_{0}}\right)+a\left(2 \pi \chi(M)-\limsup _{R \rightarrow+\infty} \int_{B_{R}} K\right) .
$$

In particular, if $K \in L^{1}(M)$, we can easily recover the classical Cohn-Vossen inequality [CV35]. Indeed, for every $R$ we consider the harmonic potential of $B_{R} \backslash B_{R_{0}}$, that is, the solution $\phi_{R} \in \mathcal{A}$ of $\Delta \phi_{R}=0$ on $B_{R} \backslash B_{R_{0}}$. Since $M$ is parabolic by Remark $5.19, \phi_{R} \rightarrow 0$ uniformly with all its derivatives on compact sets as $R \rightarrow+\infty$, and integrating by parts

$$
\int_{B_{R} \backslash B_{R_{0}}}\left|\nabla \phi_{R}\right|^{2}=\int_{B_{R} \backslash B_{R_{0}}}\left|\nabla\left(1-\phi_{R}\right)\right|^{2}=\int_{\partial B_{R_{0}}} \frac{\partial\left(1-\phi_{R}\right)}{\partial \nu} \longrightarrow 0
$$


as $R \rightarrow+\infty$. Hence, by Lebesgue dominated convergence theorem and the definition of $L$-capacity, $\operatorname{cap}_{L}\left(M \backslash B_{R_{0}}\right)=0$, thus (5.71) becomes

$$
\int_{M} K \leq 2 \pi \chi(M)-\frac{\widetilde{c}_{\beta}}{a} v^{*} \leq 2 \pi \chi(M) .
$$

It should be stressed that the Cohn-Vossen inequality holds for every complete Riemann surface with finite topology and $K \in L^{1}(M)$. The excess $2 \pi \chi(M)-\int_{M} K$ has been the subject of an intensive research, aiming to relate it to isoperimetric constants of the ends (A. Huber [Hub57], R. Finn [Fin65], A.L. Werner [Wer68]), to the volume ratio of spheres and balls (P. Hartman [Har64a] and K. Shiohama [Shi85]) and to the behaviour of Busemann functions (K. Shiohama [Shi84]).

REMARK 5.21. If $L$ is stable, with minor modifications inequality (5.70) can be improved to

$$
\widetilde{c}_{\beta} \frac{\operatorname{vol}\left(B_{r / 2}\right)}{(r / 2)^{2}}+\int_{B_{r}} V \psi_{r}^{2} \leq 2 a \pi \chi(M)+o(1) .
$$

Indeed, it is enough to set $R_{0}=R=0$ and to define $\psi_{r}(x)=f_{r}(r(x))$ in (5.66). Therefore, letting $r \rightarrow+\infty$ we deduce

$$
\widetilde{c}_{\beta} v^{*}+\int_{M} V \leq 2 a \pi \chi(M) .
$$

The next Lemma is a calculus exercise, see [RS01].

LEMMA 5.22.

$$
\text { If } \frac{r}{\operatorname{vol}\left(B_{r}\right)} \notin L^{1}(+\infty), \quad \text { then } \quad \frac{1}{\operatorname{vol}\left(\partial B_{r}\right)} \notin L^{1}(+\infty) \text {. }
$$

We are ready to prove the next Corollary. Some of the implications have already been proved in a paper of R. Gulliver [Gul88] when the ambient manifold is real analytic.

Corollary 5.23. Let $\varphi: M^{2} \rightarrow N^{3}$ be a complete, non-compact surface with constant mean curvature $H$ in an oriented 3-dimensional manifold $N$ having nonnegative scalar curvature $\bar{s}$. Suppose that $M$ has finite stability index. Then, $M$ has finite topology and

(i) If $H \neq 0$, then $\operatorname{vol}(M)<+\infty$.

(ii) If $H=0$, then

$$
\operatorname{vol}\left(B_{r}\right)=O\left(r^{2}\right) \quad \text { as } r \rightarrow+\infty, \quad \bar{s} \circ \varphi,|I I|^{2} \in L^{1}(M)
$$

and

$$
\limsup _{r \rightarrow+\infty} \int_{B_{r}} K>-\infty .
$$

In particular, if $K^{+} \in L^{1}(M)$, then $K \in L^{1}(M)$.

Proof. Since, by (5.60), the stability operator is

$$
L=-\Delta-\left(\frac{4 H^{2}+\bar{s}+|I I|^{2}}{2}\right)+K
$$

It is enough to apply Proposition 5.18 with the choices $V=\left(4 H^{2}+\bar{s}+|I I|^{2}\right) / 2$ and $a=1$ to get that $M$ has finite topology, $0 \leq V \in L^{1}(+\infty)$ and $\operatorname{vol}\left(B_{r}\right)=O\left(r^{2}\right)$, that is, (5.73) when $H=0$. If $H \neq 0$, from $V \in L^{1}(+\infty)$ we deduce that necessarily 
$\operatorname{vol}(M)<+\infty$. By Lemma 5.22, from $\operatorname{vol}\left(B_{r}\right)=O\left(r^{2}\right)$ we obtain $\left(\operatorname{vol}\left(\partial B_{r}\right)\right)^{-1} \notin$ $L^{1}(+\infty)$. Hence, from (ii) of Theorem 5.15, improved according to Remark 5.7 and applied to the stability operator with the choice $f(r)=\operatorname{vol}\left(\partial B_{r}\right)$, we deduce that

$$
\liminf _{r \rightarrow+\infty} \int_{B_{r}}(V-K)<+\infty
$$

thus the inequality in (5.74) follows. If $K^{+} \in L^{1}(M)$, then $K^{-} \in L^{1}(M)$ for otherwise $\int_{B_{r}} K \rightarrow-\infty$, which contradicts (5.74). Hence, $K \in L^{1}(M)$.

We now examine the case $H=0$ a little bit further. It should be stressed that $K^{+} \in L^{1}(M)$ follows from simple arguments once we sharpen the assumption that $N$ has non-negative scalar curvature to the higher demanding request $\overline{\text { Ricc }} \geq 0$. Indeed, if $N$ has non-negative Ricci curvature, by (5.57) and (5.73) we get also

$$
0 \leq 2 K^{+}=s^{+} \leq \bar{s}+|I I|^{2} \in L^{1}(M) .
$$

We thus have the following result, that should be compared with Corollary 1 of [FC85].

Corollary 5.24 ([FC85], Corollary 2.1 and [Gul86]). Let $\varphi: M^{2} \rightarrow N^{3}$ be a complete, non-compact minimal surface in an oriented 3-dimensional manifold $N$ satisfying $\overline{\text { Ricc }} \geq 0$. Suppose that $M$ has finite stability index. Then, $M$ has finite topology, $\operatorname{vol}\left(B_{r}\right)=O\left(r^{2}\right)$ and

$$
\overline{\operatorname{Ricc}}(\nu, \nu), \bar{s} \circ \varphi,|I I|^{2}, K \in L^{1}(M)
$$

Using item $(i)$ of Theorem 5.15 we also recover the following celebrated result of M.P. Do Carmo and C.K. Peng [CP79], D. Fischer-Colbrie and R. Schoen [FCS80] and A.V. Pogorelov [Pog81].

TheORem 5.25. Any complete, non-compact, stable minimal surface $\varphi: M \rightarrow$ $N$ of a 3-manifold with $\overline{\text { Ricc }} \geq 0$ is totally geodesic, has non-negative sectional curvature and $\overline{\operatorname{Ricc}}(\nu, \nu)=0$ on $M$. Moreover, if $N$ is Ricci flat, $M$ is flat. In particular, there exist no complete, non-compact stable minimal surfaces in any 3 manifold $N$ whose Ricci tensor is positive in the complementary of a set of $(m-1)$ Hausdorff measure zero.

Proof. By Corollary 5.23 and Lemma 5.22, $\left(\operatorname{vol}\left(\partial B_{r}\right)\right)^{-1} \notin L^{1}(+\infty)$. Hence, by Proposition 5.15, item $(i)$ with

$$
f(r)=\operatorname{vol}\left(\partial B_{r}\right), \quad q(x)=\overline{\operatorname{Ricc}}(\nu, \nu)+|I I|^{2} \geq 0
$$

we deduce that necessarily

$$
\overline{\operatorname{Ricc}}(\nu, \nu)+|I I|^{2} \equiv 0 .
$$

Since both terms are non-negative, $M$ is totally geodesic and $\overline{\operatorname{Ricc}}(\nu, \nu) \equiv 0$. In par-

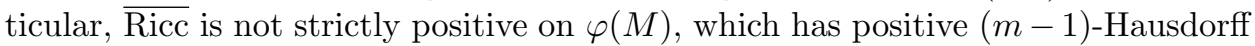
measure since $\varphi$ is an immersion. It follows that no such $M$ can exists if $\overline{\text { Ricc }}>0$ in the complementary of a set of $(m-1)$-Hausdorff measure zero. By (5.57), $2 K=\bar{s} \geq 0$, with equality sign if $N$ is Ricci flat, and this concludes the proof. 


\subsection{Newton operators, unstable hypersurfaces and the Gauss map}

In this section we shall present a recent application of our ODE results to the theory of hypersurfaces $f: M^{m} \rightarrow \mathbb{R}^{m+1}$ with some constant higher order mean curvature, [IMR11]. In this case the geometry is often suitably studied with the aid of the Newton operators. This is probably due to the fact that they are the principal part of some Jacobi operator of geometrically interesting variational integrals, see the discussion before Proposition 5.27 below. As it will be apparent in a moment, the techniques of Chapter 5 can be quite easily adapted to cover also this case. We begin with some preliminary material.

Let $f: M^{m} \rightarrow \mathbb{R}^{m+1}$ be a connected, orientable, complete, non-compact hypersurface of Euclidean space, let $\nu$ be the spherical Gauss map and denote with $A$ the shape operator in the direction of $\nu$, that is, the $(1,1)$ version of the second fundamental form. Associated with $A$ we have the principal curvatures $k_{1}, \ldots, k_{m}$ of the immersed hypersurface and the symmetric functions $S_{j}$ :

$$
S_{j}=S_{j}(k)=\sum_{i_{1}<i_{2}<\ldots<i_{j}} k_{i_{1}} k_{i_{2}} \ldots k_{i_{j}}, \quad j \in\{1, \ldots, m\}, \quad S_{0}=1,
$$

where $k=\left(k_{1}, \ldots, k_{m}\right)$. Define the $j$-mean curvature of $f$ via the normalization

$$
H_{0}=1, \quad\left(\begin{array}{c}
m \\
j
\end{array}\right) H_{j}=S_{j}
$$

Thus, for instance, $H_{1}$ is the mean curvature and $H_{m}$ is the Gauss-Kronecker curvature of the hypersurface. Note that, when changing the orientation $\nu$, the odd curvatures change sign, while the sign of the even curvatures is an invariant of the immersion. By Gauss equations (5.56) and flatness of $\mathbb{R}^{m+1}$ it is easy to see that

$$
H_{2}=\left(\begin{array}{c}
m \\
2
\end{array}\right)^{-1} S_{2}=\frac{1}{2}\left(\begin{array}{c}
m \\
2
\end{array}\right)^{-1} s(x)
$$

where $s(x)$ is the scalar curvature of $M$. The $j$-mean curvatures satisfy the so-called Newton inequalities

$$
H_{j}^{2} \geq H_{j-1} H_{j+1},
$$

equality holding if and only if $p$ is an umbilical point (see [HLP52]). We stress that no restriction is made on the sign of $H_{j-1}, H_{j}, H_{j+1}$. Furthermore, by Gärding inequalities [Gँ̈9] we have

$$
H_{1} \geq H_{2}^{1 / 2} \geq \ldots \geq H_{j}^{1 / j}
$$

on the connected component of

$$
\Gamma_{j}=\left\{k=\left(k_{1}, \ldots, k_{m}\right) \in \mathbb{R}^{m}: H_{j}(k)>0\right\}
$$

that contains the positive cone $C=\left\{k \in \mathbb{R}^{m}: k_{i}>0 \forall i\right\}$ (see [HL95] for more information). We call this component $\Gamma_{j}^{+}$. As a consequence, if $H_{j}>0$ for some $j \in\{1, \ldots, m\}$ and $k \in \Gamma_{j}^{+}$, by Gärding inequalities $H_{i}>0$ for each $1 \leq i \leq j$. Repeated applications of Newton inequalities give

$$
H_{1} H_{i+1}-H_{i+2} \geq 0 \quad \text { on } \Gamma_{j}^{+}, \quad \forall i \in\{0, \ldots, j-1\} .
$$


Indeed, the case $j=1$ comes directly from (5.77), while the case $j>1$ follows inductively by using (5.77) again:

$$
H_{1} H_{i+1}=H_{1} H_{i} \frac{H_{i+1}}{H_{i}} \geq H_{i+1} \frac{H_{i+1}}{H_{i}} \geq H_{i+2} .
$$

The Newton tensors $P_{j}, j \in\{0, \ldots, m\}$, are inductively defined by

$$
P_{0}=I, \quad P_{j}=S_{j} I-A P_{j-1},
$$

and satisfy the following algebraic properties.

Lemma 5.26 ([BC97]). Let $\left\{e_{i}\right\}$ be the principal directions associated with A, that is, $A e_{i}=k_{i} e_{i}$, and let $S_{j}\left(A_{i}\right)$ be the $j$-th symmetric function of $A$ restricted to the $(m-1)$-dimensional space $e_{i}^{\perp}$. Set $S_{m+1} \equiv 0$. Then, for each $1 \leq j \leq m-1$,

$$
\begin{aligned}
& \text { (1) } A P_{j}=P_{j} A ; \\
& \text { (2) } P_{j} e_{i}=S_{j}\left(A_{i}\right) e_{i} ; \\
& \text { (3) } \operatorname{Tr}\left(P_{j}\right)=\sum_{i} S_{j}\left(A_{i}\right)=(m-j) S_{j} ; \\
& \text { (4) } \operatorname{Tr}\left(A P_{j}\right)=\sum_{i} k_{i} S_{j}\left(A_{i}\right)=(j+1) S_{j+1} ; \\
& \text { (5) } \operatorname{Tr}\left(A^{2} P_{j}\right)=\sum_{i} k_{i}^{2} S_{j}\left(A_{i}\right)=S_{1} S_{j+1}-(j+2) S_{j+2} .
\end{aligned}
$$

From (2) of the above lemma, and the definition of $P_{m}$, it follows that $P_{m}=0$. To each $j$-th Newton tensor we associate a well defined, symmetric differential operator $L_{j}$, acting on $C_{c}^{\infty}(M)$ by setting

$$
L_{j} u=\operatorname{Tr}\left(P_{j} \operatorname{Hess} u\right) \quad \forall u \in C_{c}^{\infty}(M),
$$

Note that, since $f: M \rightarrow \mathbb{R}^{m+1}, A$ is a Codazzi tensor. Thus $L_{j}$ can be written in divergence form, precisely

$$
L_{j} u=\operatorname{div}\left(P_{j} \nabla u\right),
$$

see [CY75], [Ros93]. $L_{j}$ naturally appears when looking for stationary points of the curvature integral

$$
\mathcal{A}_{j}(M)=\int_{M} S_{j} \mathrm{~d} V_{M}
$$

for compactly supported variations that, for $j \geq 1$, are required to preserve the volume. It has been first noticed by R.C. Reilly in [Rei73] that the stationary points of $\mathcal{A}_{j}$ are characterized as those immersions having constant $S_{j+1}$, which generalize the case $j=0$ of constant mean curvature immersions. Afterwards, in [BC97] and [Elb02] the authors computed the second variation of $\mathcal{A}_{j}$ in ambient spaces even more general than space forms. For this latter, they obtained for the Jacobi operator the expression

$$
T_{j}=L_{j}+\left(S_{1} S_{j+1}-(j+2) S_{j+2}\right)=L_{j}+\operatorname{Tr}\left(A^{2} P_{j}\right) .
$$

The last equality follows from property (4) of Lemma 5.26. Since, for $j=0$,

$$
S_{1}^{2}-2 S_{2}=\left(\sum_{i} k_{i}\right)^{2}-2 \sum_{i<j} k_{i} k_{j}=\left(\sum_{i} k_{i}\right)^{2}-\left[\left(\sum_{i} k_{i}\right)^{2}-\sum_{i} k_{i}^{2}\right]=|I I|^{2},
$$

$T_{0}=\Delta+|I I|^{2}$ is the classical stability operator for minimal and CMC hypersurfaces. In general, $L_{j}$ is not elliptic. However, there are a number of sufficient conditions to guarantee this fact, and the next four are suitable for our applications. 
Proposition 5.27. Let $M$ be an m-dimensional connected, orientable hypersurface of some space form $N$.

(i) ([HL95]) Suppose that $S_{j+1} \equiv 0$. Then, $L_{j}$ is elliptic if and only if $\operatorname{rank}(A)>j$.

(ii) ([HL95], [HL99]) Suppose that $S_{j+1} \equiv 0$. Then, $L_{i}$ is elliptic for every $1 \leq i \leq j$ provided that $\operatorname{rank}(A)>j$, and that there exists a point $p \in M$ satisfying $H_{i}(p)>0$ for every $1 \leq i \leq j$.

(iii) ([BC97]) If $M$ has an elliptic point, that is, a point $p \in M$ at which $A$ is definite, and $S_{j+1} \neq 0$ at every point of $M$, then each $L_{i}, 1 \leq i \leq j$ is elliptic.

(iv) ([Elb02]) If $H_{2}>0$ on $M$, then both $L_{1}$ and $L_{2}$ are elliptic.

Furthermore, we can choose the orientation in such a way that

in (ii), $H_{i}>0$ on $M$ for every $1 \leq i \leq j$;

in (iii), $H_{i}>0$ on $M$ for every $i \in\{1, \ldots, m-1\}$;

in (iv), $H_{1}>0$ on $M$.

Remark 5.28. Condition (ii) deserves some comment. Indeed, under the assumption $S_{j+1} \equiv 0$, by $(i) L_{j}$ is elliptic, thus $P_{j}$ is definite on $M$. Since $H_{j}(p)>0$, it follows that $P_{j}$ is positive definite at $p$ and hence on the whole $M$. Thus, by (1) and (5) of Lemma 5.26,

$$
0<\operatorname{Tr}\left(A^{2} P_{j}\right)=-(j+2) S_{j+2},
$$

thus $S_{j+2}<0$ on $M$. Now, $p$ satisfies $H_{j+2}(p)<0<H_{i}(p)$ for $1 \leq i \leq j$. By an algebraic lemma ([HL99], Lemma 1.2), this is equivalent to say that the curvature vector $k(p)$ belongs to $\partial \Gamma_{j}^{+}$. A connectedness argument, together with the rank condition, shows that $k(q) \in \partial \Gamma_{j}^{+}$for every $q \in M$, which is a sufficient condition for each $L_{i}, 1 \leq i \leq j$ to be elliptic. See [HL95], [HL99] for more details.

We are now ready to prove the following

TheOREM 5.29. Let $f: M \rightarrow \mathbb{R}^{m+1}$ be a connected, complete orientable hypersurface such that, for some $j \in\{0, m-2\}, H_{j+1}$ is a non-zero constant. If $j=1$, assume that $\mathrm{H}_{2}>0$ on $M$ or, if $j \geq 2$, assume that there exists a point $p \in M$ at which the second fundamental form is definite. In both cases, choose the orientation given by the spherical Gauss map $\nu$ in such a way that $H_{i}>0$ for every $1 \leq i \leq j$. Set

$$
v_{j}(r)=(m-j)\left(\begin{array}{c}
m \\
j
\end{array}\right) \int_{\partial B_{r}} H_{j}=(m-j) \int_{\partial B_{r}} S_{j}, \quad v_{1}(r)=(m-1) \int_{\partial B_{r}} S_{1} .
$$

Fix an equator $E \subset \mathbb{S}^{m}$ and suppose that either

$$
\begin{array}{ll}
\text { (i) } \quad v_{j}(r)^{-1} \notin L^{1}(+\infty) \quad \text { and } \quad H_{1} \notin L^{1}(M) \text { or } \\
\text { (ii) } v_{j}(r)^{-1} \in L^{1}(+\infty) \quad \text { and } \\
\liminf _{r \rightarrow+\infty} \sqrt{v_{1}(r) v_{j}(r)} \int_{r}^{+\infty} \frac{\mathrm{d} s}{v_{j}(s)}>\frac{1}{2}\left[\frac{\left(\begin{array}{c}
m-2 \\
j
\end{array}\right) H_{j+1}}{m-j-1}\right]^{-1 / 2} .
\end{array}
$$

Then, there exists a divergent sequence $\left\{x_{k}\right\} \subset M$ such that $\nu\left(x_{k}\right) \in E$. 
Proof. Clearly, the possibility of choosing the orientation of $M$ in such a way that $H_{i}$ is positive for every $1 \leq i \leq j$ follows from Proposition 5.27. Fix an equator $E$ of $\mathbb{S}^{m}$ and assume, by contradiction, that there exists a sufficiently large geodesic ball $B_{r_{0}}$ such that, outside $B_{r_{0}}, \nu$ does not meet $E$. In other words, $\nu\left(M \backslash B_{r_{0}}\right)$ is contained in the open spherical caps determined by $E$. Indicating with $a \in \mathbb{S}^{m}$ one of the two focal points of $E,\langle a, \nu(x)\rangle \neq 0$ on $M \backslash B_{r_{0}}$. Let $\mathcal{C}$ be one of the connected components of $M \backslash B_{r_{0}}$; then, $\nu(\mathcal{C})$ is a subset of only one of the spherical caps. Up to replacing $a$ with $-a$, we can suppose $u=\langle a, \nu\rangle>0$ on $\mathcal{C}$. Proceeding in the same way for each connected component we can construct a positive, smooth function $u$ on $M \backslash B_{r_{0}}$. A computation due to R.C. Reilly [Rei73], H. Rosenberg [Ros93], H. Alencar and A.G. Colares [AC98], shows that, for a general immersion $f: M^{m} \rightarrow \mathbb{R}^{m+1}$

$$
T_{j}\langle a, \nu\rangle=-\left\langle\nabla S_{j+1}, a\right\rangle .
$$

Hence $u$ turns out, by the constancy of $S_{j+1}$, to be a positive solution of $T_{j} u=0$ on $M \backslash B_{r_{0}}$. Since $T_{j}$ is elliptic by (iii) of Proposition 5.27, an application of Theorem 1.33 gives $\lambda_{1}^{-T_{j}}\left(M \backslash B_{r_{0}}\right) \geq 0$. We shall now show that the assumptions of the theorem contradict this fact. Towards this aim, we first note that, since $H_{j}>0$, $v_{j}(r)$ satisfy the assumptions of (V1). Taking into account Lemma 5.26, for $r \geq r_{0}$ we define

$$
A(r)=\frac{1}{v_{j}(r)} \int_{\partial B_{r}}\left(S_{1} S_{j+1}-(j+2) S_{j+2}\right)=\frac{1}{v_{j}(r)} \int_{\partial B_{r}} \operatorname{Tr}\left(A^{2} P_{j}\right) .
$$

Then, $A(r) \geq 0$ since, in our assumptions, $P_{j}$ is positive definite. Furthermore, $A(r)$ satisfy (A1), hence by Remark 3.3 there exists $z \in \operatorname{Lip}_{\text {loc }}\left(\left[r_{0},+\infty\right)\right)$ solving

$$
\left\{\begin{array}{l}
\left(v_{j}(r) z^{\prime}(r)\right)^{\prime}+A(r) v_{j}(r) z(r)=0 \quad \text { on }\left(r_{0},+\infty\right) \\
z\left(r_{0}\right)=z_{0}>0
\end{array}\right.
$$

and $z$ has isolated zeroes. Using (5.78)

$$
\begin{aligned}
& S_{1} S_{j+1}-(j+2) S_{j+2}=m\left(\begin{array}{c}
m \\
j+1
\end{array}\right) H_{1} H_{j+1}-(j+2)\left(\begin{array}{c}
m \\
j+2
\end{array}\right) H_{j+2}= \\
& =\left(\begin{array}{c}
m \\
j+1
\end{array}\right)\left(m H_{1} H_{j+1}-(m-j-1) H_{j+2}\right) \\
& \geq\left(\begin{array}{c}
m \\
j+1
\end{array}\right)(j+1) H_{1} H_{j+1} \geq 0,
\end{aligned}
$$

so that

$$
A(r) v_{j}(r) \geq(j+1)\left(\begin{array}{c}
m \\
j+1
\end{array}\right) H_{j+1} \int_{\partial B_{r}} H_{1}=\frac{\left(\begin{array}{c}
m-2 \\
j
\end{array}\right) H_{j+1}}{m-j-1} v_{1}(r) .
$$

If $1 / v_{j} \notin L^{1}(+\infty)$, then under (5.81), $(i)$, and by the coarea formula we deduce $A v_{j} \notin L^{1}\left(\mathbb{R}^{+}\right)$. Hence, we can apply (5.16) of Theorem 5.6 to deduce that every solution of (5.84) is oscillatory. The same conclusion holds when $1 / v_{j} \in L^{1}((1,+\infty))$. Indeed, combining (5.81), (ii), and the lower bound (5.86), condition (iii) of Proposition 5.9 is satisfied with the choice $f(r)=v_{j}(r)$. Let now $R<R_{1}<R_{2}$ be two consecutive zeros of $z(r)$ after $R$. Defining $\psi(x)=z(r(x))$ on the annulus $B_{R_{2}} \backslash B_{R_{1}}$ 
and zero on the complementary set, by the coarea formula and the definition of $A(r)$ we deduce

$$
\int_{M}\left(S_{1} S_{j+1}-(j+2) S_{j+2}\right) \psi^{2}=\int_{R_{1}}^{R_{2}} z^{2}(s) A(s) v_{j}(s) \mathrm{d} s=(m-j) \int_{M} S_{j} A(r) \psi^{2} .
$$

Thus, by property (3) of Lemma 5.26, the above identity and the coarea formula, integrating by parts we deduce that

$$
\begin{aligned}
\left(-T_{j} \psi, \psi\right)_{L^{2}} & =\int_{M}\left\langle P_{j} \nabla \psi, \nabla \psi\right\rangle-\left(S_{1} S_{j+1}-(j+2) S_{j+2}\right) \psi^{2} \\
& \leq \int_{M} \operatorname{Tr}\left(P_{j}\right)|\nabla \psi|^{2}-\left(S_{1} S_{j+1}-(j+2) S_{j+2}\right) \psi^{2}= \\
& =(m-j) \int_{M} S_{j}\left[|\nabla \psi|^{2}-A(r) \psi^{2}\right] \\
& =\int_{R_{1}}^{R_{2}}\left[\left(z^{\prime}(s)\right)^{2}-A(s) z^{2}(s)\right] v_{j}(s) \mathrm{d} s \\
& =-\int_{R_{1}}^{R_{2}}\left[\left(v_{j}(s) z^{\prime}(s)\right)^{\prime}+A(s) v_{j}(s) z(s)\right] z(s) \mathrm{d} s=0 .
\end{aligned}
$$

Therefore, by the domain monotonicity $\lambda_{1}^{-T_{j}}\left(M \backslash B_{r_{0}}\right)<0$, and we reached the desired contradiction.

REMARK 5.30. As a matter of fact, the orientability of $M$ is not needed. If $M$ is non orientable, $\nu$ is not globally defined. However, changing the sign of $\nu$ does not change either the assumptions or the conclusion of Theorem 5.29, since the antipodal map on $\mathbb{S}^{m}$ leaves each $E$ fixed. If $\langle a, \nu\rangle \neq 0$ on $M \backslash B_{r_{0}}$, the normal field $X=\langle a, \nu\rangle \nu$ is nowhere vanishing and globally defined on $M \backslash B_{r_{0}}$. This shows that, in any case, every connected component of $M \backslash B_{r_{0}}$ is orientable.

We clarify the role of $(i)$ and (ii) of Theorem 5.29 with some examples. First, we deal with the case $j \neq 1$, and we assume that $v_{j}$ is of order $r^{k}$ (resp. $e^{k r}$ ) as $r \rightarrow+\infty$, for some $k>0$. Then assumption (ii) requires that $v_{1}(r)$ is of order at least $r^{k-2}$ (resp. $e^{k r}$ ). Roughly speaking, $v_{1}$ has to be large enough with respect to $v_{j}$. Under additional requirements on the intrinsic curvatures of $M$, the volume comparison Theorem 1.24 allows us to control the volume of $\partial B_{r}$ and (ii) can be read as $H_{1}$ not decaying too fast at infinity (with respect to $H_{j}$ ). When $j=1$, that is, when $M$ has constant scalar curvature $s$, by (5.76) and the definition of the critical curve $\chi_{v_{1}}$ condition (ii) reads as

$$
\liminf _{r \rightarrow+\infty} \frac{1}{\sqrt{\chi_{v_{1}}(r)}}=2 \liminf _{r \rightarrow+\infty} v_{1}(r) \int_{r}^{+\infty} \frac{\mathrm{d} s}{v_{1}(s)}>H_{2}^{-1 / 2}=\sqrt{\frac{m(m-1)}{s}} .
$$

In some sense, $v_{1}(r)$ does not have to grow too fast. Loosely speaking, by the estimates for the critical curve in Section 3.2, $v_{1}(r)$ shall not grow faster than exponentially. This shows that condition (ii) requires the balancing of two opposite effects. The same happens for $(i)$ with $j=1$. Indeed, this is a consequence of the Cauchy-Schwarz inequality and of the coarea formula:

$$
\left(\int_{R}^{r} \frac{\mathrm{d} s}{v_{1}(s)}\right)\left(\int_{B_{r} \backslash B_{R}} H_{1}\right) \geq \frac{(r-R)^{2}}{m(m-1)} .
$$


Finally, we stress that $(i)$ and $(i i)$ are mild hypotheses as they only involve the integral of extrinsic curvatures at infinity. In particular, no pointwise control is required.

Given the hypersurface $f: M^{m} \rightarrow \mathbb{R}^{m+1}$ we shall now identify the image of the tangent space at $p \in M$ with the affine hyperplane passing through $f(p)$ in the standard way. We have the following result:

THEOREM 5.31. Let $f: M \rightarrow \mathbb{R}^{m+1}$ be a complete, connected orientable hypersurface with $H_{j+1} \equiv 0$, for some $j \in\{0, \ldots, m-2\}$. If $j \geq 1$, assume that $\operatorname{rank}(A)>j$ at every point. Furthermore, if $j$ is even, suppose that there exists $p \in M$ such that $H_{j}(p)>0$. Define $v_{j}$ as in (5.80), and set

$$
s_{j+2}(r)=\int_{\partial B_{j}}\left|S_{j+2}\right| .
$$

If either

$$
\begin{aligned}
& \text { (i) } \quad\left|v_{j}(r)\right|^{-1} \notin L^{1}(+\infty) \quad \text { and } \quad H_{j+2} \notin L^{1}(M) \quad \text { or } \\
& \text { (ii) } \quad\left|v_{j}(r)\right|^{-1} \in L^{1}(+\infty) \quad \text { and } \\
& \quad \liminf _{r \rightarrow+\infty} \sqrt{s_{j+2}(r)\left|v_{j}(r)\right|} \int_{r}^{+\infty} \frac{\mathrm{d} s}{\left|v_{j}(s)\right|}>\frac{1}{2} \sqrt{\frac{1}{j+2}},
\end{aligned}
$$

then for every compact set $K \subset M$ we have

$$
\bigcup_{p \in M \backslash K} T_{p} M \equiv \mathbb{R}^{m+1},
$$

that is, the tangent envelope of $M \backslash K$ coincides with $\mathbb{R}^{m+1}$.

Proof. We start observing that we can assume that $v_{j}$ is positive on $\mathbb{R}^{+}$. Indeed, in our assumptions, by $(i)$ of Proposition 5.27 the matrix $P_{j}$ is either positive definite or negative definite everywhere. Thus, (3) of Lemma 5.26 implies that either $H_{j}>0$ or $H_{j}<0$ on $M$. If $j$ is odd, we can change the orientation of $M$ in such a way that $H_{j}$ is positive, whence $v_{j}>0$ on $\mathbb{R}^{+}$. On the other hand, if $j$ is even, this trick cannot be used and we have to rely on the existence of $p \in M$ with $H_{j}(p)>0$ to deduce that $v_{j}>0$ on $\mathbb{R}^{+}$. Applying (5) of Lemma 5.26 we obtain

$$
0<\operatorname{Tr}\left(A^{2} P_{j}\right)=-(j+2) S_{j+2}, \quad \text { hence } S_{j+2}<0 \text { on } M .
$$

Now, suppose by contradiction that, for some $K$, the tangent envelope of $M \backslash K$ does not coincide with $\mathbb{R}^{m+1}$. By choosing cartesian coordinates appropriately, we can assume that the origin 0 satisfy

$$
0 \notin \bigcup_{p \in M \backslash K} T_{p} M .
$$

Then, the function $u=\langle f, \nu\rangle$ is nowhere vanishing and smooth on $M \backslash K$. Furthermore, we can arrange the sign of $u$ on each connected component in such a way that $u>0$ on $M \backslash K$. Again, by a computation in [Rei73], [Ros93] and [AC98],

$$
T_{j}( \pm u)= \pm\left[-(j+1) S_{j+1}-\left\langle\nabla S_{j+1}, f\right\rangle\right]=0 .
$$


Note that here the assumption $H_{j+1} \equiv 0$ is essential. It follows that $\lambda_{1}^{-T_{j}}(M \backslash K) \geq$ 0 . Defining

$$
0<A(r)=\frac{1}{v_{j}(r)} \int_{\partial B_{r}} \operatorname{Tr}\left(A^{2} P_{j}\right)=-(j+2) \frac{1}{v_{j}(r)} \int_{\partial B_{r}} S_{j+2}=(j+2) \frac{s_{j+2}(r)}{v_{j}(r)},
$$

under assumptions $(i)$ or $(i i)$ the $\operatorname{ODE}\left(v_{j} z^{\prime}\right)^{\prime}+A v_{j} z=0$ is oscillatory. To show this fact, we rest upon the same oscillation criteria used in the proof of Theorem 5.29. The rest of the proof is identical to that of Theorem 5.29.

REMARK 5.32. In the statement of Theorem 5.31, we have excluded the case $j=m-1$ since for $j=m-1$ our requirements cannot be met. Indeed, condition $H_{j+1}=0$ is the vanishing of the Gauss-Kronecker curvature, which implies that the second fundamental form is everywhere singular. Therefore, $\operatorname{rank}(A)>m-1$ is automatically false.

Remark 5.33. Again, according to Remark 5.30 we can drop the orientability assumption on $M$. Indeed, if the tangent envelope of $M \backslash K$ does not cover $\mathbb{R}^{m+1}$, the vector field $X=\langle f, \nu\rangle \nu$ is a globally defined, nowhere vanishing normal vector field on $M \backslash K$, hence $M \backslash K$ is orientable.

REMARK 5.34. In the same set of assumptions of Theorem 5.31, we can prove a version of Theorem 5.29 that deals with the case $H_{j+1} \equiv 0$ on $M$.

We mention that the problem of determining the tangent envelope of an isometric immersion $M \hookrightarrow \mathbb{R}^{m+1}$ has been addressed by B. Halpern [Hal71] when $M$ is compact and orientable. More precisely, he proved that

$$
\bigcup_{x \in M} T_{x} M \not \equiv \mathbb{R}^{m+1}
$$

if and only if $M$ is embedded as the boundary of an open star-shaped domain of $\mathbb{R}^{m+1}$. Some years later, H. Alencar and K. Frensel [AF91] extended this result when the ambient manifold is a space form. In case $M$ is non-compact there are many examples satisfying (5.90), for instance cylinders over suitable curves. However, if $M$ is minimal, then $M$ is totally geodesic provided (5.90) is true and the tangent envelope is closed in $\mathbb{R}^{m+1}$, as shown in [AF91]. When $m=2$, things are more restrictive. In fact, T. Hasanis and D. Koutroufiotis in [HK84] have proved that the only complete minimal surfaces in $\mathbb{R}^{3}$ for which $(5.90)$ holds are planes. Note that the original proof of Hasanis-Koutroufiotis theorem is a consequence of (5.89) and Theorem 5.25. Indeed, if

$$
\bigcup_{x \in M} T_{x} M \not \equiv \mathbb{R}^{3}
$$

then by formula (5.89), case $j=0, u=\langle f, \nu\rangle>0$ turns out to solve $\Delta u+|I I|^{2} u=0$ on $M$. Hence, $M$ is stable on $\mathbb{R}^{3}$, thus totally geodesic.

Our last result is a splitting theorem for constant mean curvature (CMC) hypersurfaces whose Gauss map is enclosed in a sufficiently small region. We begin with the following

Definition 5.35. Let $b, m \in \mathbb{N}, 1 \leq b \leq m$, and let $\left\{w_{\alpha}\right\}, \alpha \in\{1, \ldots, b\}$ be a set of orthogonal unit vectors of $\mathbb{S}^{m} \subset \mathbb{R}^{m+1}$. We define the (closed) $b$-cup, 
$\mathcal{C}\left(\left\{w_{\alpha}\right\}\right) \subset \mathbb{S}^{m}$, as the set

$$
\mathcal{C}\left(\left\{w_{\alpha}\right\}\right)=\left\{v \in \mathbb{S}^{m}:\left\langle v, w_{\alpha}\right\rangle \geq 0 \text { for every } \alpha \in\{1, \ldots, b\}\right\} .
$$

Clearly, a 1-cup is a closed hemisphere. Before stating the theorem we recall that, having fixed a compact set $K$, each connected component of $M \backslash K$ is called an end of $M$. By a compactness argument, it can be proved that the number of ends of $M \backslash K$ is finite.

TheOrem 5.36 (Splitting and codimension reduction). Let $\varphi:\left(M^{m}, g\right) \rightarrow$ $\mathbb{R}^{m+1}$ be a connected, complete, oriented CMC hypersurface with spherical Gauss map $\nu$. Define

$$
\bar{q}(r)=\frac{1}{\operatorname{vol}\left(\partial B_{r}\right)} \int_{\partial B_{r}}|I I|^{2} .
$$

Assume that $\operatorname{vol}\left(\partial B_{r}\right) \leq f(r)$, for some $f(r) \in L_{\mathrm{loc}}^{\infty}\left(\mathbb{R}_{0}^{+}\right)$such that $f^{-1} \in L_{\mathrm{loc}}^{\infty}\left(\mathbb{R}^{+}\right) \cap$ $L^{1}(+\infty)$, and that

$$
\limsup _{r \rightarrow+\infty} \int_{R}^{r}\left(\sqrt{\bar{q}(s)}-\sqrt{\chi_{f}(s)}\right) \mathrm{d} s=+\infty
$$

for some $R>0$. Suppose that there exist $b \in\{1, \ldots, m\}$ and a compact set $K$ such that, for every end $E$ of $M \backslash K, \nu(E)$ is a subset of some b-cup (possibly depending on $E)$. Then,

(1) $\nu(M)$ is a subset of some totally geodesic $\mathbb{S}^{m-b} \subset \mathbb{S}^{m}$, where

$$
\mathbb{S}^{m-b}=\mathbb{S}^{m} \cap<\left\{w_{\alpha}\right\}>^{\perp}
$$

for some set of orthonormal vectors $\left\{w_{\alpha}\right\}_{\alpha=1}^{b} \subset \mathbb{R}^{m+1}$.

(2) There exists a totally geodesic $(m-b)$-submanifold $\Sigma_{0} \subset M$ such that $(M, g)$ is isometric to

$$
\Sigma_{0} \times \mathbb{R}^{b}, \quad \text { with the product metric } g_{\mid \Sigma_{0}}+\langle,\rangle_{\mathbb{R}^{b}} ;
$$

(3) The composition of $\varphi$ with the isometry in item (2) can be written as

$$
\widetilde{\varphi}:(p, t) \in \Sigma_{0} \times \mathbb{R}^{b} \longmapsto \varphi(p)+t^{\alpha} w_{\alpha},
$$

where $\left\{w_{\alpha}\right\}$ is the set in item (1). Furthermore, $\varphi$ maps $\Sigma_{0}$ into the affine subspace $W=\varphi\left(p_{0}\right)+\left\langle\left\{w_{\alpha}\right\}>^{\perp}\right.$ for some (hence any) $p_{0} \in \Sigma_{0}$, and $\varphi: \Sigma_{0} \rightarrow W$ has mean curvature $H$.

REmark 5.37. Condition (5.91) is satisfied, for instance, when $M$ has mean curvature $H \neq 0$ and

$$
\limsup _{r \rightarrow+\infty} \frac{\log \operatorname{vol}\left(\partial B_{r}\right)}{r}=v^{*}<\frac{2|H|}{\sqrt{m}}
$$

(in particular, when $H \neq 0$ and $M$ has subexponential volume growth). Indeed, by Newton inequality $m|I I|^{2} \geq H^{2}$ and thus $\bar{q}(r) \geq H^{2} / m$ for every $r \in \mathbb{R}^{+}$. On the other hand, by (5.92) for each $a \in\left(v^{*}, 2|H| / \sqrt{m}\right)$ there exists a constant $C>0$ such that $\operatorname{vol}\left(\partial B_{r}\right) \leq C \exp (a r)=f(r)$. Since $\chi_{f}(r) \equiv a^{2} / 4$, we get

$$
\int_{R}^{r}\left(\sqrt{\bar{q}(s)}-\sqrt{\chi_{f}(s)}\right) \mathrm{d} s \geq\left(\frac{|H|}{\sqrt{m}}-\frac{a}{2}\right)(r-R) \rightarrow+\infty
$$

as $r \rightarrow+\infty$. Thus, under (5.92), $M$ is a cylinder over an $(m-b)$-dimensional submanifold $\Sigma_{0}$ provided that the Gauss map of the ends of $M$ is enclosed in $b$-cups. 
Viceversa, CMC immersions of cylinders often satisfy (5.91). As an example, consider a compact, $(m-b)$-dimensional hypersurface $\Psi: \Sigma_{0} \rightarrow \mathbb{R}^{m-b+1}$ with constant mean curvature $H \neq 0$, and the product CMC immersion

$$
\varphi=\Psi \times \text { id }: \Sigma_{0} \times \mathbb{R}^{b} \longrightarrow \mathbb{R}^{m+1}=\mathbb{R}^{m-b+1} \times \mathbb{R}^{b} .
$$

Then, again by Newton inequality $\bar{q}(r) \geq H^{2} / m$, and moreover (since $\Sigma_{0}$ is compact) $v(r) \leq C r^{\max \{b, 2\}}$ for some $C>0$. Whence, computing $\chi_{f}$, the validity of (5.91) is immediately checked.

Proof of Theorem 5.36. First, by (ii) of Theorem 5.15 and assumption (5.91) we deduce that

$$
\operatorname{ind}_{L}(M)=+\infty, \quad \text { where } L=-\Delta-|I I|^{2} .
$$

Let $\left\{E_{1}, \ldots, E_{t}\right\}$ be the ends with respect to $K$. For each $j \in\{1, \ldots, t\}$, let $\mathcal{C}\left(\left\{w_{\alpha, j}\right\}_{\alpha}\right)$ be the $b$-cup containing $\nu\left(E_{j}\right)$, and define $u_{\alpha, j}=\left\langle\nu, w_{\alpha, j}\right\rangle$ on $M$. By formula (5.82), in our assumptions

$$
u_{\alpha, j} \in C^{\infty}(M), \quad \Delta u_{\alpha, j}+|I I|^{2} u_{\alpha, j}=0 \quad \text { on } M, \quad u_{\alpha, j} \geq 0 \text { on } E_{j} .
$$

Define $u_{\alpha}$ on $M \backslash K$ by setting $u_{\alpha}(x)=u_{\alpha, j}(x)$ if $x \in E_{j}$. Now, the equivalence $($ iii $) \Leftrightarrow(v)$ in Theorem 1.41 and (5.94) imply that necessarily $u_{\alpha} \equiv 0$ on $M \backslash K$, that is, $u_{\alpha, j} \equiv 0$ on $E_{j}$ for every $j$. By the unique continuation principle [Aro57], $u_{\alpha, j} \equiv 0$ on $M$, that is,

$$
\nu(x) \perp<\left\{w_{\alpha, j}\right\}_{\alpha, j}>\quad \text { for every } x \in M .
$$

The dimension of the vector space $Z=<\left\{w_{\alpha, j}\right\}_{\alpha, j}>$ is at least $b$, since $\left\{w_{\alpha, j}\right\}_{\alpha}$ is an orthonormal set for each $j$. Therefore, we can choose a collection of at least $b$ orthonormal vectors $\left\{w_{\alpha}\right\} \subset Z$ such that $\nu \perp<\left\{w_{\alpha}\right\}>$. If $\mathbb{S}^{m-b}$ is the totally geodesic $(m-b)$-sphere determined by

$$
\mathbb{S}^{m} \cap<\left\{w_{\alpha}\right\}>^{\perp},
$$

item (1) is proved.

To show (2), let $q \in M$ and let $U_{q}$ be a neighbourhood of $q$ such that $\varphi_{\mid U_{q}}$ is an embedding. Since $\nu \perp w_{\alpha}$, we deduce

$$
w_{\alpha} \in T_{\varphi(q)} \varphi(M) .
$$

Therefore, since $\varphi$ is a smooth isometric diffeomorphism between $U_{q}$ and $\varphi\left(U_{q}\right)$, the definition

$$
X_{\alpha}(q)=\varphi_{*, \varphi(q)}^{-1}\left(w_{\alpha}\right)
$$

is well posed and gives rise to an orthonormal set of smooth vector fields $\left\{X_{\alpha}\right\}$ on $M$. We are going to prove that the distribution

$$
\mathcal{D}: q \in M \longmapsto \mathcal{D}(q)=<X_{\alpha}(q)>^{\perp}
$$

is integrable. To do so, we prove that the associated ideal

$$
\operatorname{ker}(\mathcal{D})=\left\{\eta \in T^{*} M: \eta(v)=0 \forall v \in \mathcal{D}\right\} .
$$

is a differential ideal. Through the Gram-Schmidt procedure we can find, locally in some neighbourhood $U \subset M$, a set $\left\{e_{i}\right\} \subset T M, i \in\{1, \ldots, m-b\}$ such that $\left\{e_{i}, X_{\alpha}, \nu\right\}$ is a Darboux frame for $\varphi$, that is, $\left\{\varphi_{*} e_{i}, \varphi_{*} X_{\alpha}\right\}$ is an orthonormal basis of $T \varphi(U)$. Note that $\varphi_{*} X_{\alpha}=w_{\alpha}$, and define for notational convenience $\xi_{i}=\varphi_{*} e_{i}$. Denote with $\left\{\theta^{i}, \theta^{\alpha}, \theta^{m+1}\right\}$ the coframe dual to $\left\{\xi_{i}, w_{\alpha}, \nu\right\}$, and with $\left\{\omega_{c}^{a}\right\}, 1 \leq a, c \leq m+1$ the connection forms of $\mathbb{R}^{m+1}$. If, as usual, we omit writing 
the pullback $\varphi^{*},\left\{\theta^{1}, \theta^{\alpha}\right\}$ is an orthonormal conframe on $M$, its connection forms are $\left\{\omega_{B}^{A}\right\}, 1 \leq A, B \leq m, \theta^{m+1}=0$ and $\operatorname{ker}(\mathcal{D})$ is the ideal generated by $\left\{\theta^{\alpha}\right\}$. From the equation

$$
0=\mathrm{d} w_{\alpha}=\omega_{\alpha}^{i} \xi_{i}+\omega_{\alpha}^{\beta} w_{\beta}+\omega_{\alpha}^{m+1} \nu
$$

we argue $0=\omega_{\alpha}^{i}=\omega_{\alpha}^{\beta}=\omega_{\alpha}^{m+1}$. Hence, by the structure equations

$$
\mathrm{d} \theta^{\alpha}=-\omega_{j}^{\alpha} \wedge \theta^{j}-\omega_{\beta}^{\alpha} \wedge \theta^{\beta}-\omega_{m+1}^{\alpha} \wedge \theta^{m+1}=0 \in \mathcal{I},
$$

as desired. In the same way, every distribution $X_{\alpha}^{\perp}$ is integrable. Denote with $\Sigma_{0}$ the maximal leaf of $\mathcal{D}$ passing through some $p_{0} \in M$. From

$$
L_{X_{\alpha}} g=L_{\varphi_{*}^{-1} w_{\alpha}} \varphi^{*}\langle,\rangle=\varphi_{*}^{-1}\left(L_{w_{\alpha}}\langle,\rangle\right)=0,
$$

each $X_{\alpha}$ is a Killing vector field. From $\left|X_{\alpha}\right|=1$ and the completeness of $M$, the flow $\Phi^{\alpha}$ generated by $X_{\alpha}$ is defined on the whole $\mathbb{R} \times M$. This can be seen as follows: suppose by contradiction that there exists a maximal integral curve $\gamma:\left[0, t_{0}\right) \rightarrow M$ of $X_{\alpha}$ such that $t_{0}<+\infty$. Then, by standard theory, $\gamma$ eventually lies outside every compact set. Since $M$ is complete, $r(\gamma(t)) \rightarrow+\infty$ as $t \rightarrow t_{0}$. From

$$
r(\gamma(t))-r(\gamma(0))=\int_{0}^{t}\left\langle\nabla r, \gamma^{\prime}(s)\right\rangle \mathrm{d} s \leq \int_{0}^{t_{0}}\left|X_{\alpha}(\gamma(s))\right| \mathrm{d} s=t_{0}
$$

this necessarily implies $t_{0}=+\infty$, a contradiction. If we set

$$
\Psi^{\alpha}:(t, x) \in \mathbb{R} \times \mathbb{R}^{m+1} \longmapsto x+t w_{\alpha},
$$

by standard theory and the definition of $X_{\alpha}$ the commutation $\varphi \circ \Phi_{t}^{\alpha}=\Psi_{t}^{\alpha} \circ \varphi$ holds on $M$ for every $t \in \mathbb{R}$. Since

$$
\left[X_{\alpha}, X_{\beta}\right]=\left[\varphi_{*}^{-1}\left(w_{\alpha}\right), \varphi_{*}^{-1}\left(w_{\beta}\right)\right]=\varphi_{*}^{-1}\left[w_{\alpha}, w_{\beta}\right]=0,
$$

the vector fields $\left\{X_{\alpha}\right\}$ pairwise commutes. Thus, by standard theory, $\Phi_{s}^{\alpha} \circ \Phi_{t}^{\beta}=$ $\Phi_{t}^{\beta} \circ \Phi_{s}^{\alpha}$ for every $\alpha, \beta, s, t$. Furthermore, $X_{\alpha}$ is invariant under the flows $\left\{\Phi^{\beta}\right\}$. This follows immediately since $w_{\alpha}$ is invariant under the flows $\left\{\Psi^{\beta}\right\}$ on $\mathbb{R}^{m+1}$. We define the following map

$$
\begin{array}{rlrl}
\phi: & \Sigma_{0} \times \mathbb{R}^{b} & \longrightarrow & M \\
(p, t) & \longmapsto \Phi_{t^{b}}^{b} \circ \Phi_{t^{b-1}}^{b-1} \circ \ldots \circ \Phi_{t^{1}}^{1}(p),
\end{array}
$$

where $t=\left(t^{1}, \ldots, t^{m}\right)$. We prove that $\phi$ is a diffeomorphism. First, $\phi$ is injective. Indeed, suppose by contradiction that

$$
\Phi_{s^{b}}^{b} \circ \Phi_{s^{b-1}}^{b-1} \circ \ldots \circ \Phi_{s^{1}}^{1}(q)=\Phi_{t^{b}}^{b} \circ \Phi_{t^{b-1}}^{b-1} \circ \ldots \circ \Phi_{t^{1}}^{1}(p)
$$

for some $(q, s) \neq(p, t)$. Then, if $s=t$, applying to both terms the composition of diffeomorphisms $\left(\Phi_{t_{b}}^{b} \circ \Phi_{t^{b-1}}^{b-1} \circ \ldots \circ \Phi_{t^{1}}^{1}\right)^{-1}$ we obtain $q=p$, contradicting $(q, s) \neq$ $(p, t)$. Suppose now that $s \neq t$. Up to renaming the coordinates, we can assume that $s^{b} \neq t^{b}$. Then, setting

$$
\widetilde{q}=\Phi_{s^{b-1}}^{b-1} \circ \ldots \circ \Phi_{s^{1}}^{1}(q), \quad \widetilde{p}=\Phi_{t^{b-1}}^{b-1} \circ \ldots \circ \Phi_{t^{1}}^{1}(p)
$$

and applying $\Phi_{-t^{b}}^{b}$ to $(5.95)$ we obtain $\widetilde{p}=\Phi_{s^{b}-t^{b}}^{b}(\widetilde{q})$, so that

$$
\varphi(\widetilde{p})=\varphi \circ \Phi_{s^{b}-t^{b}}^{b}(\widetilde{q})=\Psi_{s^{b}-t^{b}}^{b} \circ \varphi(q)=\varphi(q)+\left(s^{b}-t^{b}\right) w_{p} .
$$

By their very definition, $\widetilde{p}$ and $\widetilde{q}$ belong to some maximal leaf of the distribution $X_{b}^{\perp}$. Since $p, q \in \Sigma_{0}, \widetilde{p}$ and $\widetilde{q}$ belongs to the same leaf $\Sigma$, and can therefore be connected 
by some curve $\sigma \subset \Sigma$. From $g\left(\sigma^{\prime}, X_{b}\right)=0$ for every value of the parameter, the curve $\varphi \circ \sigma$ has tangent vector always orthogonal to $w_{b}$, hence

$$
\varphi(\widetilde{q}) \in \varphi(\widetilde{p})+w_{b}^{\perp} .
$$

This contradicts (5.96) and $s^{b} \neq t^{b}$. Next, we show that $\phi_{*}$ is a diffeomorphism. By dimensional consideration, it is enough to show that $\phi_{*}$ is injective. Let $(p, t)$ be a point of $\Sigma_{0} \times \mathbb{R}^{b}$, and denote with

$$
j_{t}: \Sigma_{0} \rightarrow \Sigma_{0} \times \mathbb{R}^{b}, \quad j_{p}: \mathbb{R}^{b} \rightarrow \Sigma_{0} \times \mathbb{R}^{b}
$$

the standard inclusions. If $\partial_{\alpha}$ is the partial derivative with respect to $t^{\alpha}$, from $\phi_{*}\left(\partial_{\alpha}\right)=X_{\alpha}$ we deduce that $\phi_{*}$ is injective on $\left(j_{p}\right)_{*}\left(T \mathbb{R}^{b}\right)$. Furthermore, from the commutativity of the diagram

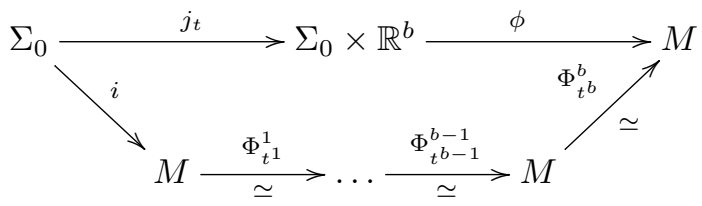

we deduce

$$
\operatorname{rank}\left(\left(\phi \circ j_{t}\right)_{*}\right)=\operatorname{rank}\left(\left(\Phi_{t^{b}}^{b} \circ \ldots \circ \Phi_{t^{1}}^{1} \circ i\right)_{*}\right)=\operatorname{rank}\left(i_{*}\right)=m-b=\operatorname{rank}\left(\left(j_{t}\right)_{*}\right) .
$$

Therefore, $\phi_{*}$ is injective also on $\left(j_{t}\right)_{*}\left(T \Sigma_{0}\right)$. Let $(V, Y) \in T\left(\Sigma_{0} \times \mathbb{R}^{b}\right)=T \Sigma_{0} \oplus T \mathbb{R}^{b}$ be such that $\phi_{*}(V, Y)=0$. Then,

$$
0=\phi_{*}(V, Y)=\phi_{*}\left(\left(j_{t}\right)_{*} V+\left(j_{p}\right)_{*} Y\right)=\phi_{*}\left(j_{t}\right)_{*} V+\phi_{*}\left(j_{p}\right)_{*} Y .
$$

From the properties of the flows $\left\{\Phi^{\alpha}\right\}$, it is not hard to show that

$$
\left[\left(\phi \circ j_{t}\right)_{*}\left(T \Sigma_{0}\right)\right] \cap\left[\left(\phi \circ j_{p}\right)_{*}\left(T \mathbb{R}^{b}\right)\right]=\{0\},
$$

thus in (5.99) we must have $\phi_{*}\left(j_{t}\right)_{*} V=\phi_{*}\left(j_{p}\right)_{*} Y=0$. Since $\phi_{*}$ is injective on $\left(j_{p}\right)_{*}\left(T \mathbb{R}^{b}\right)$ and on $\left(j_{t}\right)_{*}\left(T \Sigma_{0}\right), V=0$ and $Y=0$. This proves that $\phi_{*}$ is injective. By the implicit function theorem, $\phi$ is a local diffeomorphism and an open map. Being injective, $\phi$ is a global diffeomorphism between $\Sigma_{0} \times \mathbb{R}^{b}$ and its image, which is an open subset of $M$. The last step is to show that $\phi$ is, in fact, surjective. Since $M$ is connected, it is enough to show that $\phi\left(\Sigma_{0} \times \mathbb{R}^{b}\right)$ is closed.

Towards this aim, we first claim that the image $S_{t}=\phi \circ j_{t}\left(\Sigma_{0}\right)$ is a whole maximal slice of $\mathcal{D}$. Let $p_{1} \in \Sigma_{0}$, define $q_{1}=\phi \circ j_{t}\left(p_{1}\right)$ and let $\Sigma_{t}$ be the maximal slice containing $q_{1}$. To show that $S_{t} \subset \Sigma_{t}$, let $q_{2} \in S_{t}$ and define $p_{2} \in \Sigma_{0}$ in such a way that $q_{2}=\phi \circ j_{t}\left(p_{2}\right)$. Then, let $\gamma:[0,1] \rightarrow \Sigma_{0}$ be a curve from $p_{1}$ to $p_{2}$, and define $\sigma=\phi \circ j_{t} \circ \gamma:[0,1] \rightarrow S_{t}$. From the diagram (5.98), and since each $X_{\alpha}$ is a Killing field invariant under the flows $\Phi_{s}^{\beta}$, we can write

$$
\begin{aligned}
g\left(\sigma^{\prime}, X_{\alpha}\right) & =g\left(\left(\Phi_{t^{b}}^{b} \circ \ldots \circ \Phi_{t^{1}}^{1} \circ i \circ \gamma\right)^{\prime}, X_{\alpha}\right)=g\left(\left(\Phi_{t^{b}}^{b} \ldots \ldots \circ \Phi_{t^{1}}^{1}\right)_{*}(i \circ \gamma)^{\prime}, X_{\alpha}\right) \\
& =g\left((i \circ \gamma)^{\prime}, X_{\alpha}\right)=0,
\end{aligned}
$$

hence $\sigma$ is contained in the maximal slice $\Sigma_{t}$, thus by the arbitrariness of $q_{2}=$ $\sigma(1)$ we get $S_{t} \subset \Sigma_{t}$. To prove the converse, if by contradiction $S_{t}$ is properly contained we can choose some $q \in \Sigma_{t} \backslash S_{t}$. Now, pick a segment $\sigma \subset \Sigma_{t}$ from a point $q_{1} \in S_{t}$ to $q$. Applying $\Phi_{-t^{b}}^{b} \circ \ldots \circ \Phi_{-t^{1}}^{1}$ to $\sigma$ we would have a curve $\gamma$ from some point $p_{1} \in \Sigma_{0} \subset M$ to $p=\Phi_{-t^{b}}^{b} \circ \ldots \circ \Phi_{-t^{1}}^{1}(q)$. Proceeding analogously to 
(5.100), we deduce $\gamma^{\prime} \perp X_{\alpha}$ for every $\alpha$, hence $\gamma \subset \Sigma_{0}$. Therefore, $p \in \Sigma_{0}$ and $q=\Phi_{t^{b}}^{b} \circ \ldots \circ \Phi_{t^{1}}^{1}(p) \in S_{t}$, against our assumption. This proves the claim.

To show that $\phi$ is surjective, let

$$
q \in \overline{\phi\left(\Sigma_{0} \times \mathbb{R}^{b}\right)}
$$

and let $\Upsilon$ be the maximal slice of the distribution $\mathcal{D}$ containing $q$. Then, as above we can construct $\widetilde{\phi}: \Upsilon \times \mathbb{R}^{b} \rightarrow M$ which is a diffeomorphism with open image $\widetilde{\phi}\left(\Upsilon \times \mathbb{R}^{b}\right)$. From (5.101), necessarily $\phi\left(\Sigma_{0} \times \mathbb{R}^{b}\right)$ and $\widetilde{\phi}\left(\Upsilon \times \mathbb{R}^{b}\right)$ have nonempty intersection, that is, there exist $p_{0} \in \Sigma_{0}, p_{1} \in \Upsilon$ and suitable $s, t \in \mathbb{R}^{b}$ such that

$$
\phi\left(p_{1}, t\right)=\Phi_{t^{b}}^{b} \circ \ldots \circ \Phi_{t^{1}}^{1}\left(p_{1}\right)=\Phi_{s^{b}}^{b} \circ \ldots \circ \Phi_{s^{1}}^{1}\left(p_{2}\right)=\widetilde{\phi}\left(p_{2}, s\right),
$$

so that

$$
\Upsilon \ni p_{2}=\Phi_{t^{b}-s^{b}}^{b} \circ \ldots \circ \Phi_{t^{1}-s^{1}}^{1}\left(p_{1}\right)=\phi\left(p_{1}, t-s\right) .
$$

Since $\phi\left(\Sigma_{0}, t-s\right)$ is the whole slice $\Sigma_{t-s}, \Upsilon \equiv \Sigma_{t-s}$ and from $q \in \Upsilon$ we deduce

$$
q \in \Sigma_{t-s} \subset \phi\left(\Sigma_{0} \times \mathbb{R}^{b}\right),
$$

as claimed. We are left with the Riemannian part of the splitting. Let $h=\phi^{*} g$ be the metric on $\Sigma_{0} \times \mathbb{R}^{b}$. We can choose $\left\{e_{i}, \partial_{\alpha}\right\}$ as a basis of $\Sigma_{0} \times \mathbb{R}^{b}$, where $\left\{e_{i}\right\}$ is an orthonormal basis for $\Sigma_{0}$. Let $\left\{\theta^{j}, \mathrm{~d} t^{\alpha}\right\}$ be the dual coframe. Then, the metric writes as

$$
h=h_{i j} \theta^{i} \otimes \theta^{j}+h_{i \alpha} \theta^{i} \otimes \mathrm{d} t^{\alpha}+h_{\beta j} \mathrm{~d} t^{\beta} \otimes \theta^{j}+h_{\alpha \beta} \mathrm{d} t^{\alpha} \otimes \mathrm{d} t^{\beta} .
$$

Applying to the couple of vectors $\left(e_{i}, e_{j}\right),\left(e_{i}, \partial_{\alpha}\right)$ and $\left(\partial_{\alpha}, \partial_{\beta}\right)$ and recalling that $\phi_{*}\left(\partial_{\alpha}\right)=X_{\alpha}$ it is immediate to deduce that

$$
h=\theta^{i} \otimes \theta^{i}+\mathrm{d} t^{\alpha} \otimes \mathrm{d} t^{\alpha}
$$

This also implies that $\Sigma_{0}$ is totally geodesic in $\left(\Sigma_{0} \times \mathbb{R}^{b}, h\right)$, hence in $(M, g)$. Aposteriori, $\Sigma_{0}$ is properly embedded in $M$. To prove (3), we have already observed in (5.97) that every curve in $\Sigma_{0}$ is mapped into the affine $(m+1-b)$-space

$$
W=\varphi\left(p_{0}\right)+<\left\{w_{\alpha}\right\}>^{\perp}, \quad \text { where } p_{0} \in \Sigma_{0},
$$

whence $\varphi\left(\Sigma_{0}\right) \subset W$. From the commutation $\varphi \circ \Phi^{\alpha}=\Psi^{\alpha} \circ \varphi$ we get

$\widetilde{\varphi}(p, t)=\varphi \circ \phi(p, t)=\varphi\left(\left(\Phi_{t^{b}}^{b} \circ \ldots \circ \Phi_{t^{1}}^{1}\right)(p)\right)=\left(\Psi_{t^{b}}^{b} \circ \ldots \circ \Psi_{t^{1}}^{1}\right)(\varphi(p))=\varphi(p)+t^{\alpha} w_{\alpha}$.

It is easy to see that, in the basis $\left\{e_{i}, \partial_{\alpha}\right\}$ of $T\left(\Sigma_{0} \times \mathbb{R}^{b}\right)$, the second fundamental form $\widetilde{I I}$ of $\widetilde{\varphi}$ has the block structure

$$
\widetilde{I I}=\left(\begin{array}{cc}
\left(I I\left(e_{i}, e_{j}\right)\right) & 0 \\
0 & 0
\end{array}\right),
$$

thus the mean curvature of $\varphi$ is that of the immersed hypersurface $\varphi: \Sigma_{0} \rightarrow W \simeq$ $\mathbb{R}^{m-b+1}$.

\subsection{Dealing with a possibly negative potential}

In this section we describe how to deal with the possible negativity of $A$. The search of some sharp estimates that enables us to rewrite in a general form the results of Chapter 5 for $A<0$ seems to present some technical difficulties. For this reason, we prefer to outline a general method that we shall apply in the next sections in special situations for which the sought results are particularly appealing. For instance, a case when the method is quite effective leads to the discovery of a range of Calabi type conditions for the compactness of a complete Riemannian 
manifold. We shall consider this in Section 5.7 below.

Hereafter, we require the validity of (A1), (V1), (V2), (V3), (F1) as defined at the beginning of Chapters 3 and 5 . Let $z \in \operatorname{Lip}_{\text {loc }}\left(\mathbb{R}_{0}^{+}\right)$be a solution of

$$
\left\{\begin{array}{l}
\left(v z^{\prime}\right)^{\prime}+A v z=0 \quad \text { on } \mathbb{R}^{+}, \\
z(0)=z_{0}>0
\end{array}\right.
$$

or of the analogous problem on $\left[r_{0},+\infty\right)$. According to the proof of Theorem 5.1, the function $y=-v z^{\prime} / z$ is locally Lipschitz on $\mathcal{D}=\mathbb{R}_{0}^{+} \backslash\{r: z(r)=0\}$ and solves

$$
y^{\prime}=A v+\frac{y^{2}}{v} .
$$

Choose a function $W \in L_{\mathrm{loc}}^{\infty}\left(\mathbb{R}_{0}^{+}\right)$such that

$$
W \geq 0 \quad \text { a.e. on } \mathbb{R}^{+}, \quad W+A \geq 0 \quad \text { a.e. on } \mathbb{R}^{+} \text {. }
$$

For instance, $W$ can be taken to be the negative part of $A$. To apply the results of the previous sections, we need to produce, starting from (5.102) and $W$, a solution $\widetilde{z}$ of a linear ODE of type $\left(\widetilde{v} \widetilde{z}^{\prime}\right)^{\prime}+\widetilde{A} \widetilde{v} \widetilde{z}=0$, for some new volume function $\widetilde{v}$ and some $\widetilde{A} \geq 0$. Towards this purpose, consider a solution $w(r) \in \operatorname{Lip}_{\text {loc }}$ of

$$
\left\{\begin{array}{l}
\left(v w^{\prime}\right)^{\prime}-W v w \geq 0 \quad \text { on } \mathbb{R}^{+} \\
w\left(0^{+}\right)=w_{0}>0
\end{array}\right.
$$

Note that from $\left(v w^{\prime}\right)^{\prime} \geq W v w$ we deduce $w^{\prime} \geq 0$, hence $w$ has a positive essential infimum on $\mathbb{R}_{0}^{+}$. Therefore, the function $\widetilde{z}=z / w$ is well defined on $\mathbb{R}_{0}^{+}$and solves

$$
\left\{\begin{array}{l}
\left(\left[v w^{2}\right] \widetilde{z}^{\prime}\right)^{\prime}+(A+W)\left[v w^{2}\right] \widetilde{z} \leq 0 \quad \text { on } \mathbb{R}^{+} \\
\widetilde{z}(0)=z_{0} / w_{0}>0
\end{array}\right.
$$

Setting

$$
h(r)=-\frac{\left[v(r) w^{2}(r)\right] \widetilde{z}^{\prime}(r)}{\widetilde{z}(r)}, \quad b(r)=-\frac{v(r) w^{\prime}(r)}{w(r)},
$$

a simple computation shows that

$$
h(r)=w^{2}(r)[y(r)-b(r)] \quad \text { and } h \text { satisfies } \quad h^{\prime} \geq(A+W)\left[w^{2} v\right]+\frac{h^{2}}{w^{2} v}
$$

The proofs of Theorem 5.1, Corollary 5.2 and Theorem 5.6 can be repeated verbatim to allow $A<0$ simply by replacing

$$
y \text { with } h, A \text { with } A+W, v \text { with } v w^{2} \text { and } f \text { with } f w^{2},
$$

As already observed in Remark 5.5, the inequality sign in (5.107) and (5.106) is irrelevant for the proofs of Theorem 5.1, Corollary 5.2 and Theorem 5.6.

It is worth to observe the following fact: as clearly expressed in (5.106) and (5.107), the negative part of $A$, or in other words $W$, acts to produce a weight $w^{2}$ for the manifold. We underline that this approach has already been used for the proof of Lemma 4.31, see Remark 4.34. For particular choices of $W(r)$, to express the results in a simple form one needs an explicit $w$ solving (5.105) or, at least, sharp estimates for $w$ at infinity. In the next section we will consider some special cases that shall clarify the above observations. 


\subsection{An extension of Calabi compactness criterion}

Using the method of the previous section, we are able to determine either the existence of a first zero, or the oscillatory behaviour, of a solution $g$ of $g^{\prime \prime}+K g=0$ even when $K$ is not assumed to be non-negative near infinity. As a first main consequence we have the next geometric result.

THEOREM 5.38 (Compactness with sign-changing curvature). Let $(M,\langle\rangle$,$) be$ a complete Riemannian manifold. For each unit speed geodesic $\gamma$ issuing from some fixed origin o, define

$$
K_{\gamma}(s)=\frac{\operatorname{Ricc}\left(\gamma^{\prime}, \gamma^{\prime}\right)(s)}{m-1}
$$

Assume that one of the following set of assumptions is met.

(i) The function $K_{\gamma}(s)$ satisfies

$$
K_{\gamma}(s) \geq-B^{2}\left(1+s^{2}\right)^{\alpha / 2} \quad \text { on } \mathbb{R}^{+},
$$

for some $B>0$ and $\alpha \geq-2$ possibly depending on $\gamma$. Having set

$$
0 \leq A_{\gamma}(s)=K_{\gamma}(s)+B^{2}\left(1+s^{2}\right)^{\alpha / 2},
$$

suppose also that, for some $0<S<s$ such that $A_{\gamma} \not \equiv 0$ on $[0, S]$,

$$
\begin{aligned}
\int_{S}^{s}\left(\sqrt{A_{\gamma}(\sigma)}-\sqrt{\chi_{w^{2}}(\sigma)}\right) \mathrm{d} \sigma \\
>-\frac{1}{2}\left(\log \int_{0}^{S} A_{\gamma}(\sigma) w^{2}(\sigma) \mathrm{d} \sigma+\log \int_{S}^{+\infty} \frac{\mathrm{d} \sigma}{w^{2}(\sigma)}\right),
\end{aligned}
$$

where

$$
w(s)= \begin{cases}\sinh \left(\frac{2 B}{2+\alpha}\left[(1+s)^{1+\frac{\alpha}{2}}-1\right]\right) & \text { if } \alpha \geq 0 ; \\ s^{1 / 2} I \frac{1}{2+\alpha}\left(\frac{2 B}{2+\alpha} s^{1+\frac{\alpha}{2}}\right) & \text { if } \alpha \in(-2,0) ; \\ s^{B^{\prime}} & \text { if } \alpha=-2,\end{cases}
$$

and $B^{\prime}=\left(1+\sqrt{1+4 B^{2}}\right) / 2$.

(ii) The function $K_{\gamma}(s)$ satisfies

$$
K_{\gamma}(s) \geq \frac{B^{2}}{(1+s)^{2}} \quad \text { on } \mathbb{R}^{+}
$$

for some $B \in[0,1 / 2]$ possibly depending on $\gamma$. Having set

$$
0 \leq A_{\gamma}(s)=K_{\gamma}(s)-\frac{B^{2}}{(1+s)^{2}},
$$

suppose also that, for some $0<S<s$ such that $A_{\gamma} \not \equiv 0$ on $[0, S]$, the inequality (5.109) holds with

$$
w(s)= \begin{cases}(1+s)^{B^{\prime \prime}}-(1+s)^{1-B^{\prime \prime}} & \text { if } B \in[0,1 / 2) ; \\ \sqrt{1+s} \log (1+s) & \text { if } B=1 / 2,\end{cases}
$$

and $B^{\prime \prime}=\left(1+\sqrt{1-4 B^{2}}\right) / 2$.

Then, $M$ is compact and has finite fundamental group. 
Proof. By Theorem 2.1, $M$ is compact and has finite fundamental group provided we prove that, for every $\gamma$ issuing from $o$, the solution $g$ of

$$
\left\{\begin{array}{l}
g^{\prime \prime}+K_{\gamma}(s) g=0 \\
g(0)=0, \quad g^{\prime}(0)=1
\end{array}\right.
$$

has a first zero. Note that, both for (5.110) and for (5.111), the critical curve related to $w^{2}$ exists since $1 / w^{2} \in L^{1}(+\infty)$.

(i). As observed in the proof of Theorem 3.16, the function $w$ in (5.110) is a positive solution of

$$
w^{\prime \prime}-B^{2}\left(1+s^{2}\right)^{\alpha / 2} w \geq 0 \quad \text { on } \mathbb{R}^{+}
$$

whose initial condition, in the cases $\alpha \in(-2,0)$ and $\alpha \geq 0$, is

$$
w(0)=0, \quad w^{\prime}(0)=C>0 .
$$

Consider the function $\widetilde{z}=g / w$. Then, by the previous section, $\widetilde{z}$ solves

$$
\left(w^{2} \widetilde{z}^{\prime}\right)^{\prime}+A_{\gamma} w^{2} \widetilde{z} \leq 0 \quad \text { on } \mathbb{R}^{+} .
$$

In order to apply Corollary 5.2 to the differential inequality (5.114), we shall make use of Remark 5.5. From (5.113), in each case of (5.110) we get

$$
\frac{w^{2} \widetilde{z}^{\prime}}{\widetilde{z}}\left(0^{+}\right)=\left(w^{2} \frac{g^{\prime}}{g}-w w^{\prime}\right)\left(0^{+}\right)=0 .
$$

By Remark 5.5, this initial condition enables us to apply Corollary 5.2, and the inequality (5.109) implies that $\widetilde{z}$ (hence $g$ ) has a first zero. Case (ii) is analogous. Indeed, by Remark 1.22, $w$ in (5.111) is a solution of the Cauchy problem

$$
\left\{\begin{array}{l}
w^{\prime \prime}+\frac{B^{2}}{(1+s)^{2}} w=0 \\
g(0)=0, \quad g^{\prime}(0)=C>0 .
\end{array}\right.
$$

REMARK 5.39. We recall that, by (3.22), inequality (5.109) is equivalent to the somehow simpler one

$$
\int_{S}^{s} \sqrt{A_{\gamma}(\sigma)} \mathrm{d} \sigma>-\frac{1}{2}\left(\log \int_{0}^{S} A_{\gamma}(\sigma) w^{2}(\sigma) \mathrm{d} \sigma+\log \int_{s}^{+\infty} \frac{\mathrm{d} \sigma}{w^{2}(\sigma)}\right) .
$$

In the statement of the theorem, we have preferred to use the form (5.109) to put in evidence that the RHS does not depend on $s$, as opposed to conditions like (2.15) and (2.24) where both $a$ and $b$ appear in the LHS as well as in the RHS.

We note that, for $m=3, B=1 / 2$ in (5.111), for $\alpha=0,-2$ in (5.110) and for $B=0$ in (5.111), assumption (5.116) can be further simplified. Indeed,

$$
\int_{s}^{+\infty} \frac{\mathrm{d} \sigma}{w^{2}(\sigma)}= \begin{cases}\frac{s^{-\sqrt{1+4 B^{2}}}}{\sqrt{1+4 B^{2}}} & \text { for }(5.110), \alpha=-2 \text { and for } B=0 \\ B^{-1}[\operatorname{coth}(B s)-1] & \text { for }(5.110), \alpha=0 \\ \frac{1}{\log (1+s)} & \text { for }(5.111), B=1 / 2, m=3 .\end{cases}
$$

To generalize Calabi oscillation criterion, we prove the next Proposition, which follows easily from the discussion of the previous section. 
Proposition 5.40. Suppose that

$$
K, G \in L_{\mathrm{loc}}^{\infty}\left(\mathbb{R}_{0}^{+}\right), \quad K(s) \geq-G(s) \quad \text { on }\left[s_{0},+\infty\right),
$$

for some $s_{0} \geq 0$. Let $w$ be positive solution of

$$
w^{\prime \prime}-G(s) w \geq 0 \quad \text { on }\left[s_{0},+\infty\right) .
$$

Then, any solution $g$ of $g^{\prime \prime}+K(s) g=0$ is oscillatory provided that either

$$
\frac{1}{w^{2}(s)} \notin L^{1}(+\infty) \quad \text { and } \quad(K(s)+G(s)) w^{2}(s) \notin L^{1}(+\infty)
$$

or $1 / w^{2} \in L^{1}(+\infty)$ and

$$
\limsup _{s \rightarrow+\infty}\left(\int_{s_{0}}^{s} \sqrt{K(\sigma)+G(\sigma)} \mathrm{d} \sigma+\frac{1}{2} \log \int_{s}^{+\infty} \frac{\mathrm{d} \sigma}{w^{2}(\sigma)}\right)=+\infty .
$$

ProOF. The function $\widetilde{z}=g / w$ solves

$$
\left\{\begin{array}{l}
\left(w^{2} \widetilde{z}^{\prime}\right)^{\prime}+(K+G) w^{2} \widetilde{z} \leq 0 \quad \text { on }\left[s_{0},+\infty\right) \\
\widetilde{z}\left(s_{0}\right)>0
\end{array}\right.
$$

By Remark 5.5 the inequality sign in (5.119) is irrelevant. Therefore, we can conclude by means of Theorem 5.6 and Remark 5.8 .

TheOREM 5.41 (Generalized Calabi criterion). Let $K \in L_{\mathrm{loc}}^{\infty}\left(\mathbb{R}_{0}^{+}\right)$, and let $g \not \equiv$ be a solution of $g^{\prime \prime}+K g=0$. Then, $g$ oscillates in each of the following cases:

(1) $K$ satisfies

$$
K(s) \geq-B^{2} s^{\alpha} \quad \text { when } s>s_{0}
$$

$$
\begin{aligned}
& \text { for } \alpha=-2, \quad \limsup _{s \rightarrow+\infty}\left(\int_{s_{0}}^{s} \sqrt{K(\sigma)+\frac{B^{2}}{\sigma^{2}}} \mathrm{~d} \sigma-\frac{\sqrt{1+4 B^{2}}}{2} \log s\right)=+\infty ; \\
& \text { for } \alpha>-2, \quad \limsup _{s \rightarrow+\infty}\left(\int_{s_{0}}^{s} \sqrt{K(\sigma)+B^{2} \sigma^{\alpha}} \mathrm{d} \sigma-\frac{2 B}{\alpha+2} s^{\frac{\alpha}{2}+1}\right)=+\infty .
\end{aligned}
$$

(2) K satisfies

$$
K(s) \geq \frac{B^{2}}{s^{2}} \quad \text { when } s>s_{0}
$$

$$
\begin{aligned}
& \text { for } B<\frac{1}{2}, \quad \limsup _{s \rightarrow+\infty}\left(\int_{s_{0}}^{s} \sqrt{K(\sigma)-\frac{B^{2}}{\sigma^{2}}} \mathrm{~d} \sigma-\frac{\sqrt{1-4 B^{2}}}{2} \log s\right)=+\infty \\
& \text { for } B=\frac{1}{2}, \quad \limsup _{s \rightarrow+\infty}\left(\int_{s_{0}}^{s} \sqrt{K(\sigma)-\frac{1}{4 \sigma^{2}}} \mathrm{~d} \sigma-\frac{1}{2} \log \log s\right)=+\infty
\end{aligned}
$$


Proof. (1). Set $G(s)=B^{2} s^{\alpha}$ in Proposition 5.40. Then, $w^{\prime \prime}-B^{2} s^{\alpha} w=0$ has the particular positive solution

$$
\begin{array}{ll}
w(s)=\sqrt{s} I_{\frac{1}{2+\alpha}}\left(\frac{2 B}{2+\alpha} s^{1+\frac{\alpha}{2}}\right) & \text { if } \alpha>-2 ; \\
w(s)=s^{B^{\prime}}, \quad B^{\prime}=\frac{1+\sqrt{1+4 B^{2}}}{2} \quad \text { if } \alpha=-2,
\end{array}
$$

where $I_{\nu}(s)$ is the Bessel function in (3.41). In both cases, $1 / w^{2} \in L^{1}(+\infty)$, and computing the asymptotic behaviour with the aid of (3.43) we get

$$
\int_{s}^{+\infty} \frac{\mathrm{d} \sigma}{w^{2}(\sigma)} \sim \begin{cases}C \exp \left(-\frac{4 B}{2+\alpha} s^{1+\frac{\alpha}{2}}\right) & \text { if } \alpha>-2 \\ C s^{1-2 B^{\prime}}=C s^{-\sqrt{1+4 B^{2}}} & \text { if } \alpha=-2 .\end{cases}
$$

Therefore, condition (5.121) is equivalent to (5.118), and $g^{\prime \prime}+K g=0$ is oscillatory by Proposition 5.40 .

(2). The proof is the same. Indeed, it is enough to consider the following positive solution $w$ of $w^{\prime \prime}+B^{2} s^{-2} w=0$ :

$$
\begin{array}{ll}
w(s)=s^{B^{\prime \prime}}, \quad B^{\prime \prime}=\frac{1+\sqrt{1-4 B^{2}}}{2} & \text { if } B \in[0,1 / 2) ; \\
w(s)=\sqrt{s} \log s & \text { if } B=1 / 2 .
\end{array}
$$

Again, in both cases $1 / w^{2} \in L^{1}(+\infty)$.

REMARK 5.42. Observe that setting $B=0$ in (5.123) we recover the original Calabi condition (5.28). Moreover, Theorem 5.40 also generalizes Proposition 5.11, where the case $\alpha=0$ has been proved with a different method.

Remark 5.43. Clearly, when $K \geq 0$ on $\left[s_{0},+\infty\right)$ the limitation $B \in[0,1 / 2]$ in (5.122) covers the more interesting cases. Indeed, if (5.122) is met for some $B>1 / 2$, then the oscillatory behaviour of $g$ already follows from Hille-Nehari Theorem 2.7.

Combining the technique described in this section with Theorem 4.1 and Corollary 4.4, we also obtain an improvement of Proposition 1.21.

Theorem 5.44 (Positivity and nonoscillation criteria). Let $K \in L_{\mathrm{loc}}^{\infty}\left(\mathbb{R}_{0}^{+}\right)$.

(1) Suppose that

$$
K(s) \leq \frac{1}{4(1+s)^{2}}\left[1+\frac{1}{\log ^{2}(1+s)}\right] \quad \text { on } \mathbb{R}^{+} .
$$

Then, every solution $g$ of

$$
\left\{\begin{array}{l}
g^{\prime \prime}+K g \geq 0 \\
g(0)=0, \quad g^{\prime}(0)=1
\end{array}\right.
$$

is positive on $\mathbb{R}^{+}$and satisfies $g(s) \geq C \sqrt{s \log s} \log \log s$, for some $C>0$ and for $s>3$. 
(2) Suppose that

$$
K(s) \leq \frac{1}{4 s^{2}}\left[1+\frac{1}{\log ^{2} s}\right] \quad \text { on }\left[s_{0},+\infty\right),
$$

for some $s_{0}>0$. Then, every solution $g$ of $g^{\prime \prime}+K g=0$ is nonoscillatory.

Proof. (1). By Sturm argument, it is sufficient to prove the desired conclusion under the additional assumptions that $g$ satisfies (5.127) with the equality sign, and that

$$
K(s) \geq \frac{1}{4(1+s)^{2}}
$$

Let $w(s)=\sqrt{1+s} \log (1+s)$ be the solution of $(5.127)$ with $K(s)=\left[4(1+s)^{2}\right]^{-1}$. Then, $\widetilde{z}=g / w$ solves

$$
\left\{\begin{array}{l}
\left(w^{2} \widetilde{z}^{\prime}\right)^{\prime}+\left[K(s)-\frac{1}{4(1+s)^{2}}\right] w^{2} \widetilde{z}=0 \quad \text { on } \mathbb{R}^{+}, \\
\widetilde{z}(0)=1, \quad \widetilde{z}^{\prime}(0)=0 .
\end{array}\right.
$$

Applying Theorem 4.1, $\widetilde{z}$ is positive provided

$$
K(s)-\frac{1}{4(1+s)^{2}} \leq \chi_{w^{2}}(s)=\frac{1}{4(1+s)^{2} \log ^{2}(1+s)},
$$

that is, (5.126), and $\widetilde{z}$ satisfies

$$
\widetilde{z}(s) \geq-C \sqrt{\int_{s}^{+\infty} \frac{\mathrm{d} \sigma}{w^{2}(\sigma)}} \log \int_{s}^{+\infty} \frac{\mathrm{d} \sigma}{w^{2}(\sigma)}=C \frac{\log \log s}{\sqrt{\log s}},
$$

for some $C>0$ and $s$ sufficiently large. The lower bound for $g$ follows at once by the definition of $\widetilde{z}$.

To prove (2), again by Sturm argument we can assume that the inequality $K \geq$ $1 /\left[4 s^{2}\right]$ holds. Indeed, suppose that we have shown that a solution $\widetilde{g}$ of $\widetilde{g}^{\prime \prime}+\widetilde{K} \widetilde{g}=0$ is positive on some interval $\left[s_{0},+\infty\right)$, where

$$
\widetilde{K}(s)=\max \left\{K(s), \frac{1}{4 s^{2}}\right\},
$$

and assume by contradiction that a solution $g$ of $g^{\prime \prime}+K g=0$ oscillates. Let $s_{1}, s_{2}$ be two consecutive zeroes of $g$ after $s_{0}$, chosen in such a way that $g>0$ on $\left(s_{1}, s_{2}\right)$. Then, $g$ solves $g^{\prime \prime}+\widetilde{K} g \geq 0$ on $\left[s_{1}, s_{2}\right]$. By Sturm separation Theorem 1.9, (ii), $\widetilde{g}$ should have a zero on $\left(s_{1}, s_{2}\right)$, contradiction. Proceeding along the same lines as for (1) with the choice $w(s)=\sqrt{s} \log s$, and using Corollary 4.4, we reach the desired conclusion.

REMARK 5.45. Consider the particular case

$$
K(s)=\frac{1}{4 s^{2}}+\frac{c^{2}}{4 s^{2} \log ^{2} s}, \quad \text { on }\left[r_{0},+\infty\right),
$$

for some $r_{0}>0$ and $c>0$. Then, if $c \leq 1$ Theorem 5.44 implies that $g^{\prime \prime}+K g=0$ is nonoscillatory. On the contrary, when $c>1$, by (5.123) $g^{\prime \prime}+K g=0$ is oscillatory. We observe that, on $\left[r_{0},+\infty\right)$,

$$
\frac{1}{4}<s \int_{s}^{+\infty} K(\sigma) \mathrm{d} \sigma \leq \frac{1}{4}+s \frac{c^{2}}{4 s} \int_{s}^{+\infty} \frac{\mathrm{d} \sigma}{\sigma \log ^{2} \sigma}=\frac{1}{4}+\frac{c^{2}}{4 \log s},
$$


hence the Hille-Nehari criterion cannot detect neither the oscillatory nor the nonoscillatory behaviour of $g$ depending on $c$.

The proof of Theorem 5.44 suggests an iterative procedure to improve our oscillatory and nonoscillatory criteria with an arbitrary precision. In the general case, suppose that we are given an ordinary differential equation of the type $\left(v z^{\prime}\right)^{\prime}+$ $A v z=0$, with $v$ such that $\chi$ can be defined. By Sturm argument, there is no loss of generality if we assume that $A \geq \chi$. An explicit solution $w$ of

$$
\left(v w^{\prime}\right)^{\prime}+\chi v w=0
$$

is given by

$$
w(s)=-\sqrt{\int_{s}^{+\infty} \frac{\mathrm{d} \sigma}{v(\sigma)}} \log \int_{s}^{+\infty} \frac{\mathrm{d} \sigma}{v(\sigma)},
$$

and it is positive on some intervall $\left[s_{0},+\infty\right)$. Then, $\widetilde{z}=z / w$ solves

$$
\left(\bar{v} \widetilde{z}^{\prime}\right)^{\prime}+(A-\chi) \bar{v} \widetilde{z}=0 \quad \text { on }\left[s_{0},+\infty\right),
$$

where $\bar{v}=v w^{2}$, which implies that $\widetilde{z}$, and therefore $z$, are nonoscillatory if $\left(v w^{2}\right)^{-1} \in$ $L^{1}(+\infty)$ and

$$
A(s)-\chi(s) \leq \chi_{v w^{2}}(s),
$$

and oscillatory if $\left(v w^{2}\right)^{-1} \in L^{1}(+\infty)$ and

$$
\limsup _{s \rightarrow+\infty} \int_{s_{0}}^{s}\left(\sqrt{A(\sigma)-\chi(\sigma)}-\sqrt{\chi_{v w^{2}}(\sigma)}\right) \mathrm{d} \sigma=+\infty,
$$

or equivalently if

$$
\limsup _{s \rightarrow+\infty}\left(\int_{s_{0}}^{s} \sqrt{A(\sigma)-\chi(\sigma)} \mathrm{d} \sigma+\frac{1}{2} \log \int_{s}^{+\infty} \frac{\mathrm{d} \sigma}{v(\sigma) w^{2}(\sigma)}\right)=+\infty .
$$

Now, the procedure can be pushed a step further by considering $\widetilde{z}$. This enables us to construct finer and finer critical curves. As an example, we now get a first refinement of the conditions of Theorem 5.44. Suppose that

$$
K(s) \geq \frac{1}{4 s^{2}}+\frac{1}{4 s^{2} \log ^{2} s}
$$

on, say, $[2,+\infty)$. Then, as in the proof of Theorem 5.44, define $w(s)=\sqrt{s} \log s$ and $v(s)=w(s)^{2}=s \log ^{2} s$. Since $w$ is a positive solution of $w^{\prime \prime}+\left(4 s^{2}\right)^{-1} w=0$ on some $\left[s_{1},+\infty\right), z=g / w$ is well defined and solves $\left(v z^{\prime}\right)^{\prime}+A v z=0$ on $\left[s_{1},+\infty\right)$, where

$$
A(s)=K(s)-\frac{1}{4 s^{2}} \geq \frac{1}{4 s^{2} \log ^{2} s}=\chi_{w^{2}}(s)=\chi(s) .
$$

Now, the function

$$
w_{2}(s)=-\sqrt{\int_{s}^{+\infty} \frac{\mathrm{d} \sigma}{v(\sigma)}} \log \int_{s}^{+\infty} \frac{\mathrm{d} \sigma}{v(\sigma)}=\frac{\log \log s}{\sqrt{\log s}}
$$

is a solution of $\left(v w_{2}^{\prime}\right)^{\prime}+\chi v w_{2}=0$, positive after some $s_{2} \geq s_{1}$. Setting

$$
v_{2}(s)=v(s) w_{2}(s)^{2}=s \log s \log ^{2} \log s,
$$

then

$$
\frac{1}{v_{2}(s)} \in L^{1}(+\infty)
$$


and the function $z_{2}=z / w_{2}$ is a solution of $\left(v_{2} z_{2}^{\prime}\right)^{\prime}+A_{2} v_{2} z_{2}=0$ on $\left[s_{2},+\infty\right)$, where

$$
A_{2}(s)=A(s)-\chi(s)=K(s)-\frac{1}{4 s^{2}}-\frac{1}{4 s^{2} \log ^{2} s} \geq 0 .
$$

Thus $z_{2}$, and hence $z$ and $g$, is nonoscillatory provided

$$
A_{2}(s) \leq \chi_{v_{2}}(s), \quad \text { that is }, \quad K(s) \leq \frac{1}{4 s^{2}}+\frac{1}{4 s^{2} \log ^{2} s}+\frac{1}{4 s^{2} \log ^{2} s \log ^{2} \log s},
$$

and, by (5.131), it is oscillatory if

$$
\limsup _{s \rightarrow+\infty}\left(\int_{s_{2}}^{s} \sqrt{K(\sigma)-\frac{1}{4 \sigma^{2}}-\frac{1}{4 \sigma^{2} \log ^{2} \sigma}} \mathrm{d} \sigma-\frac{1}{2} \log \log \log s\right)=+\infty .
$$

The general result that improves on Theorem 5.44 with an arbitrary degree of precision follows by means of an inductive procedure, and we leave the technical details to the interested reader.

We now observe that the explicit solutions of $w^{\prime \prime}-B^{2} s^{\alpha} w \geq 0$ can be used, via the change of variables (5.36), to produce positive, explicit solutions $\widetilde{w}$ of $(5.105)$, for suitable $W$. This trick enables us to get simple extensions of spectral estimates for Schrödinger operators, which are particularly appealing in the case of $\mathbb{R}^{m}$, see the next Theorem 5.46 .

To be more precise, let $w$ be as in (5.124), so that $w \in C^{1}\left(\left[s_{0},+\infty\right)\right)$ and

$$
w^{\prime \prime}-B^{2} s^{\alpha} w=0 .
$$

According to (5.36), choose some function $v$ satisfying, as usual, (V1) and $1 / v \in$ $L^{1}(+\infty)$, and define

$$
s(r)=\left(\int_{r}^{+\infty} \frac{\mathrm{d} \tau}{v(\tau)}\right)^{-1}, \quad \widetilde{w}(r)=\frac{w(s(r))}{s(r)} .
$$

Then, $s: \mathbb{R}^{+} \rightarrow \mathbb{R}^{+}, s\left(s_{0}\right)=r_{0}>0$, and

$$
\begin{array}{ll}
\widetilde{w}(r)=\sqrt{\int_{r}^{+\infty} \frac{\mathrm{d} s}{v(s)} I_{\frac{1}{2+\alpha}}\left(\frac{2 B}{2+\alpha}\left[\int_{r}^{+\infty} \frac{\mathrm{d} s}{v(s)}\right]^{-1-\frac{\alpha}{2}}\right)} & \text { if } \alpha>-2 ; \\
\widetilde{w}(r)=\left(\int_{r}^{+\infty} \frac{\mathrm{d} s}{v(s)}\right)^{1-B^{\prime}} & \text { if } \alpha=-2 .
\end{array}
$$

By Proposition 3.10 and the definition of $\chi, \widetilde{w}$ solves

$$
\begin{aligned}
0 & =\left(v \widetilde{w}^{\prime}\right)^{\prime}-\left[B^{2}\left(\int_{r}^{+\infty} \frac{\mathrm{d} s}{v(s)}\right)^{-\alpha-4} \frac{1}{v^{2}(r)}\right] v \widetilde{w} \\
& =\left(v \widetilde{w}^{\prime}\right)^{\prime}-\left[4 B^{2}\left(\int_{r}^{+\infty} \frac{\mathrm{d} s}{v(s)}\right)^{-\alpha-2} \chi(r)\right] v \widetilde{w} .
\end{aligned}
$$

Setting

$$
W(r)=4 B^{2}\left(\int_{r}^{+\infty} \frac{\mathrm{d} s}{v(s)}\right)^{-\alpha-2} \chi(r)
$$


we can now use the machinery described in Section 5.6 to improve Theorem 5.1, Corollary 5.2 and Theorem 5.6, together with their applications, in case $A \geq-W$ on $\left[r_{0},+\infty\right)$ by replacing

$$
\begin{aligned}
& v(r) \quad \text { with } \quad v(r) \widetilde{w}^{2}(r), \quad \text { where } \widetilde{w}(r) \text { is as in (5.132), } \\
& A(r) \quad \text { with } \quad A(r)+W(r), \quad \text { where } W(r) \text { is as in (5.133). }
\end{aligned}
$$

In particular, by Theorem 5.6 and Remark 5.8, a solution $z$ of $\left(v z^{\prime}\right)^{\prime}+A v z=0$ is oscillatory whenever $1 /\left[\widetilde{w}^{2} v\right] \in L^{1}(+\infty)$ and

$$
\limsup _{r \rightarrow+\infty}\left(\int_{r_{0}}^{r} \sqrt{A(s)+W(s)} \mathrm{d} s+\frac{1}{2} \log \int_{r}^{+\infty} \frac{\mathrm{d} s}{\widetilde{w}^{2}(s) v(s)}\right)=+\infty .
$$

From the geometric point of view, it would be desirable to substitute $W(r)$ with

$$
W_{f}(r)=4 B^{2}\left(\int_{r}^{+\infty} \frac{\mathrm{d} s}{f(s)}\right)^{-\alpha-2} \chi_{f}(r) .
$$

Unfortunately, we have not been able to prove a comparison result for the function $W$ similar to the one for the critical curve. For this reason, we only consider the prototype case of $\mathbb{R}^{m}, m \geq 3$, where $v(r)=f(r)=\omega_{m-1} r^{m-1}$. Note that the next theorem is a further refinement of Theorems 4.10 and 5.15, and definitely improves on a classical result of M. Reed and B. Simon [RS78], and W. Kirsch and B. Simon [KS88].

Theorem 5.46 (Index of Schrödinger operators on $\mathbb{R}^{m}$ ). Let $q(x) \in L_{\mathrm{loc}}^{\infty}\left(\mathbb{R}^{m}\right)$, $m \geq 3$, and denote with $\bar{q}(r)$ the spherical mean of $q$ on $\partial B_{r}$. Define $L=-\Delta-q(x)$.

(1) Assume that $\bar{q}(r)$ satisfy

$$
\bar{q}(r) \geq-c^{2} r^{\mu} \quad \text { on }[R,+\infty),
$$

$$
\begin{aligned}
& \mu>-2, \quad \limsup _{r \rightarrow+\infty}\left[\int_{R}^{r} \sqrt{\bar{q}(s)+c^{2} s^{\mu}} \mathrm{d} s-\frac{2 c}{\mu+2} r^{\frac{\mu+2}{2}}\right]=+\infty \\
& \mu=-2, \quad \limsup _{r \rightarrow+\infty}\left[\int_{R}^{r} \sqrt{\bar{q}(s)+c^{2} s^{-2}} \mathrm{~d} s-\frac{\sqrt{(m-2)^{2}+4 c^{2}}}{2} \log r\right]=+\infty .
\end{aligned}
$$

(2) Assume that $\bar{q}(r)$ satisfy

$$
\bar{q}(r) \geq \frac{c^{2}}{r^{2}} \quad \text { on }[R,+\infty),
$$

$$
\begin{array}{ll}
c \in\left[0, \frac{m-2}{2}\right), & \limsup _{r \rightarrow+\infty}\left[\int_{R}^{r} \sqrt{\bar{q}(s)-\frac{c^{2}}{s^{2}}} \mathrm{~d} s-\frac{\sqrt{(m-2)^{2}-4 c^{2}}}{2} \log r\right]=+\infty ; \\
c=\frac{m-2}{2}, & \limsup _{r \rightarrow+\infty}\left[\int_{R}^{r} \sqrt{\bar{q}(s)-\frac{(m-2)^{2}}{4 s^{2}}} \mathrm{~d} s-\frac{1}{2} \log \log r\right]=+\infty .
\end{array}
$$


(3) Suppose that

$$
q(x) \leq \frac{(m-2)^{2}}{4 r(x)^{2}}\left[1+\frac{1}{\log ^{2}\left((m-2) r(x)^{m-2}\right)}\right] .
$$

Then L has finite index.

Proof. Reasoning as in (ii) of Theorem 5.15, to prove (1) and (2) it is enough to guarantee that a solution $z$ of

$$
\left(r^{m-1} z^{\prime}\right)^{\prime}+A r^{m-1} z=0, \quad \text { where } A=\bar{q}
$$

oscillates. We begin with proving (1). By the above discussion, $z$ oscillates provided (5.134) is met with $v(r)=r^{m-1}, \widetilde{w}$ as in (5.132) and $W$ as in (5.133). We show that, for suitable choices of $\alpha$ and $B$ in the definition of $W,(5.134)$ is equivalent to (5.135). Set

$$
\alpha=\frac{\mu-2(m-3)}{m-2}, \quad B=c(m-2)^{-\frac{2 m-2+\mu}{2(m-2)}}=c(m-2)^{-2-\frac{\alpha}{2}} .
$$

Then, $\mu \geq-2$ is equivalent to $\alpha \geq-2$,

$$
\frac{\mu+2}{2}=(m-2) \frac{2+\alpha}{2}
$$

and

$$
W(r)=4 B^{2}\left(\int_{r}^{+\infty} \frac{\mathrm{d} s}{v(s)}\right)^{-\alpha-2} \chi(r)=c^{2} r^{\mu} .
$$

As for the weight $\widetilde{w}$, from (5.132), (5.139), (5.138) and the asymptotic behaviour (3.43) we get

$$
\widetilde{w}(r) \sim \begin{cases}\sim C_{1} r^{-\frac{m-2}{2}+\frac{\mu+2}{4}} \exp \left(\frac{2 c}{2+\mu} r^{1+\frac{\mu}{2}}\right) & \text { if } \mu>-2 ; \\ C_{1} r^{(m-2)\left(B^{\prime}-1\right)} & \text { if } \mu=-2 .\end{cases}
$$

for some constant $C_{1}>0$ that may vary from line to line, hence

$$
\int_{r}^{+\infty} \frac{\mathrm{d} s}{\widetilde{w}^{2}(s) s^{m-1}} \sim \begin{cases}C_{1} \frac{1}{2 c} \exp \left(-\frac{4 c}{2+\mu} r^{1+\frac{\mu}{2}}\right) & \text { if } \mu>-2 ; \\ C_{1} r^{-(m-2)\left(2 B^{\prime}-1\right)} & \text { if } \mu=-2,\end{cases}
$$

where

$$
(m-2)\left(2 B^{\prime}-1\right)=(m-2) \sqrt{1+4 B^{2}}=\sqrt{(m-2)^{2}+4 c^{2}} .
$$

Combining (5.140), (5.141) and Remark 5.8, we get immediately that (5.134) is equivalent to (5.135).

The proof of (2) is similar. Indeed, it is enough to consider the positive solutions $w$ of $w^{\prime \prime}+B^{2} r^{-2} w=0$ in (5.125), where $B=c /(m-2)$, and to proceed as in (1). As for (3), denote with $A(r)$ the RHS of (5.137). By the procedure of Theorem 4.10 , it is enough to show that a solution $z$ of $\left(r^{m-1} z^{\prime}\right)^{\prime}+A v z=0$ is nonoscillatory. Changing variables according to Proposition 3.10:

$$
s(r)=\left(\int_{r}^{+\infty} \frac{\mathrm{d} \tau}{\tau^{m-1}}\right)^{-1}=(m-2) r^{m-2}, \quad g(s)=s z(r(s)),
$$


we obtain that $g(s)$ solves

$$
g^{\prime \prime}(s)+\frac{A(r(s)) r(s)^{2(m-1)}}{s^{4}} g(s)=0 .
$$

Since

$$
\frac{A(r(s)) r(s)^{2(m-1)}}{s^{4}}=\frac{1}{4 s^{2}}\left[1+\frac{1}{\log ^{2} s}\right],
$$

the nonoscillatory behaviour of $z$ follows from Theorem 5.44, (2) applied to $g$.

REMARK 5.47. Another extension of the classical result in [RS78], [KS88] to the case of complete Riemannian manifolds has been recently found by K. Akutagawa and H. Kumura $[\mathbf{A K}]$. Their method is very close to that used by S. Agmon in [Agm85], see also Remark 4.14. Hence, it would be interesting to investigate the interplay between their approach and the one presented in this work. In this respect, further interesting results can be found in [Kum11].

\section{Question:}

(3) Is it possible to extend Theorem 5.46 on general manifolds, without requiring the exact behaviour of $\operatorname{vol}\left(\partial B_{r}\right)$ ? 


\section{CHAPTER 6}

\section{Much above the critical curve}

In this Chapter, we consider the problem of controlling the distance between consecutive zeroes of oscillatory solutions $z \in \operatorname{Lip}_{\text {loc }}\left(\left[r_{0},+\infty\right)\right)$ of

$$
\left(v(r) z^{\prime}(r)\right)^{\prime}+A(r) v(r) z(r)=0 .
$$

For $\varrho \in\left(r_{0},+\infty\right)$, we set $R_{1}(\varrho)$ and $R_{2}(\varrho)$ to denote the first and the second zero of $z$ after $\varrho$. Our aim is to provide an upper bound, depending on $z$, of the difference $R_{2}(\varrho)-R_{1}(\varrho)$. In the first section below we prove one of our main results of the paper. The last two sections are devoted to some geometric applications, especially on the growth of the index of Schrödinger operators on balls and on the spectrum of the Laplacian on a "punctured" manifold.

\subsection{Controlling the oscillation}

We begin with some preliminary considerations. Let us assume, for the moment, that $A, v$ satisfy (V1), ( $\left.\mathrm{V}_{\mathrm{L} 1}\right),(\mathrm{A} 1)$ and $A \geq 0$ on $\left[r_{0},+\infty\right)$, for some $r_{0}>0$. In this setting, by Theorem 5.6 we know that (6.1) is oscillatory provided

$$
\limsup _{r \rightarrow+\infty} \int_{r_{0}}^{r}(\sqrt{A(s)}-\sqrt{\chi(s)}) \mathrm{d} s=+\infty,
$$

where $\chi(r)$ is the critical curve. It is reasonable to expect that larger contributions of the integral of $\sqrt{A}$ with respect to that of $\sqrt{\chi}$ near infinity produce "thicker" oscillations of $z$. As we have seen in the proof of Theorem 4.1, under the change of variables (4.6) and the definition (4.9) of $\beta(t)$, equation (6.1) transforms into

$$
\ddot{\beta}+\left\{\frac{A(r(t))}{\chi(r(t))}-1\right\} \beta=0 \quad \text { on }\left[t_{0},+\infty\right), \quad t_{0}=t\left(r_{0}\right) .
$$

We set

$$
h(t)=\frac{A(r(t))}{\chi(r(t))}-1
$$

and we suppose that

$$
A(r) \geq c^{2} \chi(r) \quad \text { on }\left[r_{0},+\infty\right),
$$

for some positive constant $c>1$. This implies $h(t) \geq c^{2}-1$, and by Sturm separation Theorem 1.9, $(i i)$, there is a zero of $\beta(t)$ between every pair of consecutive zeros of a solution $\widehat{\beta}(t)$ of

$$
\ddot{\widehat{\beta}}+\left(c^{2}-1\right) \widehat{\beta}=0 .
$$

These solutions are explicitly given by

$$
\widehat{\beta}(t)=C_{1} \cos \left(\sqrt{c^{2}-1} t\right)+C_{2} \sin \left(\sqrt{c^{2}-1} t\right) .
$$


Thus, since the distance between consecutive zeros of $\widehat{\beta}$ is $2 \pi / \sqrt{c^{2}-1}$, indicating with $T_{1}(\tau)$ and $T_{2}(\tau)$ the first pair of consecutive zeros of $\beta(t)$ after $\tau>t_{0}$, we have

$$
T_{2}(\tau)-T_{1}(\tau) \leq \frac{4 \pi}{\sqrt{c^{2}-1}},
$$

and, in particular,

$$
T_{2}(\tau)-\tau \leq \frac{6 \pi}{\sqrt{c^{2}-1}}
$$

To return to $z$ we use (4.6) and we observe that, if $\varrho=r(\tau)$, by $(4.9) r\left(T_{i}(\tau)\right)=$ $R_{i}(\varrho)$. Hence, we are led to

$$
-\frac{1}{2} \log \int_{R_{2}(\varrho)}^{+\infty} \frac{\mathrm{d} s}{v(s)}+\frac{1}{2} \log \int_{\varrho}^{+\infty} \frac{\mathrm{d} s}{v(s)} \leq \frac{6 \pi}{\sqrt{c^{2}-1}},
$$

and therefore

$$
\left\{\int_{\varrho}^{+\infty} \frac{\mathrm{d} s}{v(s)}\right\} /\left\{\int_{R_{2}(\varrho)}^{+\infty} \frac{\mathrm{d} s}{v(s)}\right\} \leq \exp \left\{\frac{12 \pi}{\sqrt{c^{2}-1}}\right\} .
$$

Now, suppose we have a good knowledge of $v(r)$, namely, something like

$$
B \exp \left\{b r^{\beta}\right\} \leq v(r) \leq A \exp \left\{a r^{\alpha}\right\}
$$

for $r>>1$ and some positive constants

$$
0<\beta \leq \alpha, b \leq a \text { if } \beta=\alpha, B \leq A \text { if } \beta=\alpha, b=a .
$$

Then, a simple computation shows that there exists a universal constant $C>0$ depending only on those in (6.5) such that

$$
\frac{1}{\varrho^{\alpha-\beta}}\left(\frac{R_{2}(\varrho)}{\varrho}\right)^{\beta-1} \exp \left\{a \varrho^{\alpha}\left[\frac{b}{a}\left(\frac{R_{2}(\varrho)}{\varrho}\right)^{\beta} \frac{1}{\varrho^{\alpha-\beta}}-1\right]\right\} \leq C
$$

for $\varrho>>1$. If $\alpha=\beta$, it is immediate to deduce

$$
\limsup _{\varrho \rightarrow+\infty} \frac{R_{2}(\varrho)}{\varrho} \leq\left(\frac{a}{b}\right)^{1 / \beta}<+\infty .
$$

However, note that for $\alpha>\beta$ conclusions of this type cannot be obtained from the previous reasoning. Furthermore, observe that the assumption

$$
v(r) \asymp \exp \left\{\Lambda r^{\alpha} \log ^{\beta} r\right\} \quad \text { as } r \rightarrow+\infty
$$

implies

$$
\lim _{\varrho \rightarrow+\infty} \frac{R_{2}(\varrho)}{\varrho}=1
$$

while, if $v(r) \asymp r^{\alpha} \log ^{\beta} r$ as $r \rightarrow+\infty$, for some $\alpha>1$ or $\alpha=1$ and $\beta>1$,

$$
\frac{R_{2}(\varrho)}{\varrho}=O(1) \quad \text { as } \varrho \rightarrow+\infty .
$$

Although the above argument is particularly elementary, in order to obtain the useful conclusions (6.8) and (6.9) we need to know the precise behaviour of $v(r)$ at infinity. In geometrical problems $v(r)$ represents $\operatorname{vol}\left(\partial B_{r}\right)$, and this latter can be estimated from above by a lower bound on the Ricci tensor, and from below by an upper bound on the sectional curvature $K$ together with the requirement that the cut-locus of the fixed origin is empty. To require all these estimates on Ricc and 
$K$ and a further matching of the two bounds on $\operatorname{vol}\left(\partial B_{r}\right)$ is a highly demanding request from the geometric point of view. We want to obtain the same kind of results on $R_{2}(\varrho)-R_{1}(\varrho)$ under the sole one-sided bound

$$
\operatorname{vol}\left(\partial B_{r}\right) \leq f(r) .
$$

This goal requires a new approach to the problem. Nevertheless, before proceeding we push the previous method a step further to better grasp the situation at hand. We observe that, to deduce (6.8), it is enough to be able to replace in (6.4) $v(r)$ with

$$
f(r)=\Lambda \exp \left\{a r^{\alpha} \log ^{\beta} r\right\}, \quad \Lambda, a, \alpha>0, \beta \geq 0 .
$$

Note that we are not requiring here $v \leq f$. An inspection of the proof of the comparison Proposition 3.12 suggests that this happens if $\chi \geq \chi_{f}$. Therefore, this yields the following

Proposition 6.1. Let $(M,\langle\rangle$,$) be a complete Riemannian manifold with a$ pole $o$ and radial sectional curvature satisfying

$$
K_{\text {rad }}(x) \leq-B^{2}\left(1+r(x)^{2}\right)^{\alpha / 2},
$$

for some $B>0$ and $\alpha>-2$. Set $v(r)=\operatorname{vol}\left(\partial B_{r}\right)$, and let $A \in L_{\mathrm{loc}}^{\infty}\left(\mathbb{R}_{0}^{+}\right)$be such that $A(r) \geq c^{2} \chi(r)$ for some $c>1$ and $r>>1$. Then, the $O D E\left(v z^{\prime}\right)^{\prime}+A v z=0$ is oscillatory and, denoting with $R_{2}(\varrho)$ the second zero of $z$ after $\varrho$,

$$
\lim _{\varrho \rightarrow+\infty} \frac{R_{2}(\varrho)}{\varrho}=1 \text {. }
$$

Proof. By the comparison for the critical curve (Proposition 3.13) $\chi \geq \chi_{g^{m-1}}$, where $g>0$ solves

$$
\left\{\begin{array}{l}
g^{\prime \prime}-B^{2}\left(1+r^{2}\right)^{\alpha / 2} g \leq 0 \\
g(0)=0, \quad g^{\prime}(0)=1
\end{array}\right.
$$

Accordingly, by (6.4) and the proof of Proposition 3.12

$$
\left\{\int_{\varrho}^{+\infty} \frac{\mathrm{d} s}{g(s)^{m-1}}\right\} /\left\{\int_{R_{2}(\varrho)}^{+\infty} \frac{\mathrm{d} s}{g(s)^{m-1}}\right\} \leq\left\{\int_{\varrho}^{+\infty} \frac{\mathrm{d} s}{v(s)}\right\} /\left\{\int_{R_{2}(\varrho)}^{+\infty} \frac{\mathrm{d} s}{v(s)}\right\} \leq C
$$

for some $C>0$. As for the proof of Proposition 3.18, explicit $g$ are given by

$$
g(r)= \begin{cases}C r^{1 / 2} I_{\frac{1}{2+\alpha}}\left(\frac{2 B}{2+\alpha} r^{1+\frac{\alpha}{2}}\right) & \text { if } \alpha \geq 0 ; \\ B^{-1} \sinh \left(\frac{2 B}{2+\alpha}\left[(1+r)^{1+\frac{\alpha}{2}}-1\right]\right) & \text { if } \alpha \in(-2,0)\end{cases}
$$

for a suitable $C>0$. Computing the asymptotic for $g^{m-1}$ with the aid of (3.43), and arguing as at the beginning of this chapter up to (6.6), it is easy to obtain (6.11).

The above theorem shows that a two-sided bound on $v(r)$ is not really necessary: the lower bound suffices. However, it should be stressed that (6.10) implies $\chi \geq$ $\chi_{g^{m-1}}$, so that assumption $A \geq c^{2} \chi$ cannot be replaced by the more manageable $A \geq c^{2} \chi_{g^{m-1}}$. This is, in some sense, the counterpart for the lack of an upper bound for $v$. If we add a corresponding upper bound for $\chi$, with an application of Theorem 3.16 we deduce the following useful 
Proposition 6.2. Let $(M,\langle\rangle$,$) be a complete Riemannian manifold of dimen-$ sion $m \geq 2$ with a pole $o$ and curvatures satisfying

$$
\begin{aligned}
K_{\mathrm{rad}}(x) & \leq-B^{2}\left(1+r(x)^{2}\right)^{\alpha / 2} \\
\operatorname{Ricc}(\nabla r, \nabla r) & \geq-(m-1) \widetilde{B}^{2}\left(1+r(x)^{2}\right)^{\widetilde{\alpha} / 2}
\end{aligned}
$$

for some $B, \widetilde{B}>0$ and $\alpha, \widetilde{\alpha}>-2$. Set $v(r)=\operatorname{vol}\left(\partial B_{r}\right)$, and let $A \in L_{\mathrm{loc}}^{\infty}\left(\mathbb{R}_{0}^{+}\right)$be such that

$$
\liminf _{r \rightarrow+\infty} \frac{A(r)}{r^{\widetilde{\alpha}}}>\frac{(m-1)^{2}}{4}
$$

Then, the $O D E\left(v z^{\prime}\right)^{\prime}+A v z=0$ is oscillatory and, denoting with $R_{2}(\varrho)$ the second zero of $z$ after $\varrho$,

$$
\lim _{\varrho \rightarrow+\infty} \frac{R_{2}(\varrho)}{\varrho}=1
$$

In Propositions 6.1 and 6.2, since $\alpha>-2$ the polynomial case for the growth of $v(r)$ is excluded; this is not an accident. With a minor modification of the arguments at the beginning of this section, we can provide a simple counterexample. Consider $v(r)=r^{m-1}$ and $A(r)=c^{2} \chi(r)$ on $\left[r_{0},+\infty\right)$, and let $z$ be a nontrivial solution of (6.1). Then, $\beta(t)$ constructed as in (4.9) solves $\ddot{\beta}+\left(c^{2}-1\right) \beta=0$ on $\left[t_{0},+\infty\right)$, so that $\beta$ has the expression (6.3). Then, there exists $C>0$ such that $T_{2}(\tau)-\tau \geq C$, and changing variables we are led to

$$
\left\{\int_{\varrho}^{+\infty} \frac{\mathrm{d} s}{s^{m-1}}\right\} /\left\{\int_{R_{2}(\varrho)}^{+\infty} \frac{\mathrm{d} s}{s^{m-1}}\right\} \geq e^{C}>1
$$

Computing the integrals we deduce

$$
\liminf _{\varrho \rightarrow+\infty} \frac{R_{2}(\varrho)}{\varrho} \geq e^{\frac{C}{m-2}}>1 .
$$

We underline another important feature of the above counterexample: the potential $A$, coinciding with a multiple of $\chi$, has the same polynomial order of decay at infinity; in fact, a quadratic decay. One may ask what happens if $A$ decays more slowly at infinity and $v(r)=r^{\alpha}$, for some $\alpha>1$. With a repeated application of Sturm separation Theorem 1.9 to the ODE (6.2), it is not hard to see that (6.11) is satisfied. The next step is to understand what happens if $v(r)$ has portions with polynomial growth, but $A$ is modelled on a curve that decay more slowly than $r^{-2}$. Towards this aim, it is worth to observe the critical curve associated to a volume function $v(r)$ that present fast oscillations between polynomial and exponential bounds. In this respect, the following example might be useful. Let $v(r)$ be defined as follows: if $n \in \mathbb{N}$,

$$
v(r)= \begin{cases}n^{3}+2\left(e^{n+\frac{1}{2}}-n^{3}\right)(r-n) & \text { if } r \in\left[n, n+\frac{1}{2}\right] ; \\ (n+1)^{3}+2\left(e^{n+\frac{1}{2}}-(n+1)^{3}\right)(n+1-r) & \text { if } r \in\left[n+\frac{1}{2}, n+1\right] .\end{cases}
$$


Then, $v(n)=n^{3}, v(n+1 / 2)=e^{n+1 / 2}$ and

$$
\begin{aligned}
& \int_{n}^{n+1} \frac{\mathrm{d} s}{v(s)}= \\
& =\frac{n+1 / 2}{2\left(e^{n+\frac{1}{2}}-n^{3}\right)}+\frac{n+1 / 2}{2\left(e^{n+\frac{1}{2}}-(n+1)^{3}\right)}-\frac{3 \log n}{2\left(e^{n+\frac{1}{2}}-n^{3}\right)}-\frac{3 \log (n+1)}{2\left(e^{n+\frac{1}{2}}-(n+1)^{3}\right)} .
\end{aligned}
$$

If $n \geq n_{0}$ and $n_{0}$ is sufficiently large, then

$$
\frac{n+1 / 2}{e^{n+\frac{1}{2}}-(n+1)^{3}} \leq \frac{n+1 / 2}{e^{n+1 / 4}} \leq \frac{1}{e^{n}}, \quad \frac{n+1 / 2}{4\left(e^{n+\frac{1}{2}}-n^{3}\right)} \geq \frac{1}{e^{n+\frac{1}{2}}} .
$$

Therefore, denoting respectively with $\lfloor x\rfloor$ the floor of $x \in \mathbb{R}$ and with $\lceil x\rceil$ the ceiling of $x$, we deduce

$$
\begin{aligned}
& \int_{r}^{+\infty} \frac{\mathrm{d} s}{v(s)} \leq \sum_{n=\lfloor r\rfloor}^{\infty} \frac{1}{e^{n}}=\frac{e}{(e-1) e^{\lfloor r\rfloor}} \leq \frac{e^{2}}{(e-1) e^{r}} \\
& \int_{r}^{+\infty} \frac{\mathrm{d} s}{v(s)} \geq \frac{1}{\sqrt{e}} \sum_{n=\lceil r\rceil}^{\infty} \frac{1}{e^{n}}=\frac{\sqrt{e}}{(e-1) e^{\lceil r\rceil}} \geq \frac{1}{\sqrt{e}(e-1) e^{r}} .
\end{aligned}
$$

Hence we finally get

$$
\int_{r}^{+\infty} \frac{\mathrm{d} s}{v(s)} \asymp \frac{1}{e^{r}} \quad \text { as } r \rightarrow+\infty .
$$

This gives that, as $r \rightarrow+\infty, \sqrt{\chi(r)}$ is is of the same order as

$$
h(r)= \begin{cases}\frac{e^{r}}{n^{3}+2\left(e^{n+\frac{1}{2}}-n^{3}\right)(r-n)} & \text { if } r \in\left[n, n+\frac{1}{2}\right] \\ \frac{e^{r}}{(n+1)^{3}+2\left(e^{n+\frac{1}{2}}-(n+1)^{3}\right)(n+1-r)} & \text { if } r \in\left[n+\frac{1}{2}, n+1\right]\end{cases}
$$

Observe that $h\left(n+\frac{1}{2}\right)=1$ for every $n \in \mathbb{N}$, while $h(n)=e^{n} / n^{3}$ quickly diverges as $n \rightarrow+\infty$. This implies that $\chi(r)$ may present high peaks where $v(r)$ has its "holes".

Now, let $f(r)$ be an upper bound for $v$, for instance $f(r)=e^{r}$. Then, the critical function modelled on the upper bound is $\chi_{f} \equiv 1 / 4$. Therefore, one cannot expect that a pointwise bound on $A$ in terms of $\chi_{f}$ could imply a pointwise control of $A$ with respect to $\chi$. However, the peaks of $h(r)$ above the function $1 / 4$ are somehow not "massive". This is a consequence of (3.22) and (6.14):

$$
\int_{R}^{r} \sqrt{\chi(s)} \mathrm{d} s=-\frac{1}{2} \log \int_{r}^{+\infty} \frac{\mathrm{d} s}{v(s)}+\frac{1}{2} \log \int_{R}^{+\infty} \frac{\mathrm{d} s}{v(s)} \sim \frac{r}{2} \sim \int_{R}^{r} \sqrt{\chi_{f}(s)} \mathrm{d} s .
$$

Since oscillations are provided under an integral control of $A$ and $\chi$, we may think that non massive peaks are negligible in estimating the distance of consecutive zeroes.

The above discussion can be summarized in the following question. Assume that $1 / v \in L^{1}(+\infty)$, and that we can control the volume only from above; for instance,

$$
v(r) \leq f(r)=\Lambda \exp \left\{a r^{\alpha} \log ^{\beta} r\right\}, \quad \Lambda, a, \alpha>0, \beta \geq 0 .
$$


Suppose that $A \geq c^{2} \chi_{f}$ for some $c>1$. By (3.34) and (3.35), this latter condition reads

$$
A(r) \geq c^{2}\left(\frac{a^{2} \alpha^{2}}{4}\right) r^{2(\alpha-1)} \log ^{2 \beta} r \sim c^{2}\left[\frac{f^{\prime}(r)}{2 f(r)}\right]^{2}=c^{2} \widetilde{\chi}_{f}(r)
$$

as $r \rightarrow+\infty$. From condition (5.17) and the non-integrability of $\sqrt{\chi_{f}}$ we know that $z$ is oscillatory. Note that the decay of $\chi_{f}$ at infinity is slower than $r^{-2}$. Do assumptions (6.15) and (6.16) imply

$$
\limsup _{\varrho \rightarrow+\infty} \frac{R_{2}(\varrho)}{\varrho} \leq C, \quad \text { or even } \quad \lim _{\varrho \rightarrow+\infty} \frac{R_{2}(\varrho)}{\varrho}=1 ?
$$

To answer this question, throughout this section we shall require the validity of the following properties on $\left[r_{0},+\infty\right)$, for some $r_{0}>0$.

$\left(\mathrm{V} 1+\mathrm{V}_{\mathrm{L} 1}\right)$

$$
0 \leq v(r) \in L_{\mathrm{loc}}^{\infty}\left(\left[r_{0},+\infty\right)\right), \quad \frac{1}{v(r)} \in L_{\mathrm{loc}}^{\infty}\left(\left[r_{0},+\infty\right)\right) \cap L^{1}(+\infty)
$$

$$
f \in C^{1}\left(\left[r_{0},+\infty\right)\right) \quad, \quad f\left(r_{0}\right)>0
$$

(F3) $\quad f$ is non decreasing on $\left[r_{0},+\infty\right)$

$$
\begin{aligned}
& v(r) \leq f(r) \quad \text { a.e. on }\left[r_{0},+\infty\right) \\
& \forall r \geq r_{0} \quad \frac{f^{\prime}(r)}{f(r)} \geq \frac{1}{D r^{\mu}} \quad \text { for some } D>0, \mu<1 \\
& A \in L_{\mathrm{loc}}^{\infty}\left(\left[r_{0},+\infty\right)\right), \quad A(r) \geq 0 \quad \text { on }\left[r_{0},+\infty\right) \\
& \limsup _{r \rightarrow+\infty} \int_{r_{0}}^{r}\left(\sqrt{A(s)}-\sqrt{\chi_{f}(s)}\right) \mathrm{d} s=+\infty \\
& \exists c>0 \text { such that } \sqrt{A(r)} \geq c \sqrt{\tilde{\chi}_{f}(r)}=\frac{c}{2} \frac{f^{\prime}(r)}{f(r)} \quad \text { on }\left[r_{0},+\infty\right)
\end{aligned}
$$

Clearly, $f$ as in (6.15) meets requirements (F2), (F3), (F5) and, by (6.16), (A4) implies (A3) when $c>1$. Furthermore, in the above assumptions, every solution $z$ of (6.1) is oscillatory by Theorem 5.6, and the zeroes of $z$ are isolated.

Next, we introduce two classes of functions: for $f \in C^{0}\left(\left[r_{0},+\infty\right)\right), f>0$ on $\left[r_{0},+\infty\right), h, k$ piecewise $C^{0}$ and non-negative on $\left[r_{0},+\infty\right), c>0$ we set

$$
\begin{aligned}
\mathcal{A}(f, h, c)= & \left\{g:\left[r_{0},+\infty\right) \rightarrow \mathbb{R}_{0}^{+} \text {piecewise } C^{0}\right. \text { such that } \\
& \left.\limsup _{r \rightarrow+\infty}\left(\sup _{\xi \in(0,1)} \frac{(1-\xi) g(r) f(r+g(r)+h(r))^{c}}{f(r+(1-\xi) g(r)+h(r))^{c+1}}\right)<+\infty\right\}
\end{aligned}
$$

$$
\begin{aligned}
\mathcal{B}(f, k, c)= & \left\{g:\left[r_{0},+\infty\right) \rightarrow \mathbb{R}_{0}^{+} \text {piecewise } C^{0}\right. \text { such that } \\
& \left.\limsup _{r \rightarrow+\infty}\left(\sup _{\xi \in(0,1)} \frac{\xi g(r) f(r+(1-\xi) g(r)+k(r))^{c}}{f(r+g(r)+k(r)) \cdot f(r+k(r))^{c}}\right)<+\infty\right\}
\end{aligned}
$$


Definition 6.3. We shall say that $f$ satisfies property $(P)$ for some $c>0$ if, whenever

$$
h(r), k(r)=O(r) \quad \text { as } \quad r \rightarrow+\infty, \quad \text { and } \quad g \in \mathcal{A}(f, h, c) \cup \mathcal{B}(f, k, c),
$$

then $g(r)=O(r)$ as $r \rightarrow+\infty$.

Lemma 6.4. The function $f(r)=\Lambda \exp \left\{a r^{\alpha} \log ^{\beta} r\right\}$, for $\Lambda, a, \alpha>0, \beta \geq 0$ satisfies property $(P)$ for every $c>1$.

Proof. Let $h$ and $k$ be non-negative and such that $h(r), k(r)=O(r)$ as $r \rightarrow+\infty$ and let $g \in \mathcal{A}(f, h, c)$. Assume, by contradiction, the existence of a sequence $\left\{r_{n}\right\} \rightarrow+\infty$ with the property

$$
\frac{g\left(r_{n}\right)}{r_{n}} \rightarrow+\infty \quad \text { as } n \rightarrow+\infty
$$

Without loss of generality we suppose $g\left(r_{n}\right)>1 \forall n$ and we define $\xi_{n}=1-\frac{1}{g\left(r_{n}\right)}$. Then

$$
\begin{aligned}
\Theta_{n}= & \frac{\left(1-\xi_{n}\right) g\left(r_{n}\right) f\left(r_{n}+g\left(r_{n}\right)+h\left(r_{n}\right)\right)^{c}}{f\left(r_{n}+\left(1-\xi_{n}\right) g\left(r_{n}\right)+h\left(r_{n}\right)\right)^{c+1}}=\frac{f\left(r_{n}+g\left(r_{n}\right)+h\left(r_{n}\right)\right)^{c}}{f\left(r_{n}+1+h\left(r_{n}\right)\right)^{c+1}} \\
= & \exp \left\{a c\left(r_{n}+g\left(r_{n}\right)+h\left(r_{n}\right)\right)^{\alpha} \log ^{\beta}\left(r_{n}+g\left(r_{n}\right)+h\left(r_{n}\right)\right)+\right. \\
& \left.-a(c+1)\left(r_{n}+1+h\left(r_{n}\right)\right)^{\alpha} \log ^{\beta}\left(r_{n}+1+h\left(r_{n}\right)\right)\right\} \\
= & \exp \left\{a c g\left(r_{n}\right)^{\alpha} \log ^{\beta}\left(r_{n}+g\left(r_{n}\right)+h\left(r_{n}\right)\right)\left[\Omega_{n}-\Sigma_{n}\right]\right\},
\end{aligned}
$$

with

$$
\begin{aligned}
& \Omega_{n}=\left(1+\frac{r_{n}}{g\left(r_{n}\right)}+\frac{h\left(r_{n}\right)}{g\left(r_{n}\right)}\right)^{\alpha} \\
& \Sigma_{n}=\frac{(c+1) r_{n}^{\alpha}}{c g\left(r_{n}\right)^{\alpha}}\left(1+\frac{1}{r_{n}}+\frac{h\left(r_{n}\right)}{r_{n}}\right)^{\alpha} \frac{\log ^{\beta}\left(r_{n}+1+h\left(r_{n}\right)\right)}{\log ^{\beta}\left(r_{n}+g\left(r_{n}\right)+h\left(r_{n}\right)\right)} .
\end{aligned}
$$

Note that $\Omega_{n} \rightarrow 1$, while $\Sigma_{n} \rightarrow 0$ as $n \rightarrow+\infty$. Their difference is thus eventually positive, so $\Theta_{n} \rightarrow+\infty$ as $r \rightarrow+\infty$, but this contradicts the fact that $g \in \mathcal{A}(f, h, c)$. Observe that here any $c>0$ would work. Similarly, we let $g \in \mathcal{B}(f, k, c)$ and we reason again by contradiction. Let $\left\{r_{n}\right\}$ be as in (6.19). Then

$$
\begin{aligned}
\Theta_{n}= & \xi g\left(r_{n}\right) \frac{f\left(r_{n}+(1-\xi) g\left(r_{n}\right)+k\left(r_{n}\right)\right)^{c}}{f\left(r_{n}+g\left(r_{n}\right)+k\left(r_{n}\right)\right) \cdot f\left(r_{n}+k\left(r_{n}\right)\right)^{c}} \\
= & \xi g\left(r_{n}\right) \exp \left\{a c(1-\xi)^{\alpha} g\left(r_{n}\right)^{\alpha}\left(1+\frac{1}{1-\xi}\left(\frac{r_{n}}{g\left(r_{n}\right)}+\frac{k\left(r_{n}\right)}{g\left(r_{n}\right)}\right)\right)^{\alpha} .\right. \\
& \log ^{\beta}\left(r_{n}+(1-\xi) g\left(r_{n}\right)+k\left(r_{n}\right)\right)-a g\left(r_{n}\right)^{\alpha}\left(1+\frac{r_{n}}{g\left(r_{n}\right)}+\frac{k\left(r_{n}\right)}{g\left(r_{n}\right)}\right)^{\alpha} . \\
& \left.\log ^{\beta}\left(r_{n}+g\left(r_{n}\right)+k\left(r_{n}\right)\right)-a c r_{n}^{\alpha}\left(1+\frac{k\left(r_{n}\right)}{r_{n}}\right)^{\alpha} \log ^{\beta}\left(r_{n}+k\left(r_{n}\right)\right)\right\} \\
\geq & \xi g\left(r_{n}\right) \exp \left\{a g\left(r_{n}\right)^{\alpha} \log ^{\beta}\left(r_{n}+(1-\xi) g\left(r_{n}\right)+k\left(r_{n}\right)\right)\left[\Omega_{n}-\Sigma_{n}\right]\right\}
\end{aligned}
$$


with

$$
\begin{aligned}
\Omega_{n} & =\left(c(1-\xi)^{\alpha}-\frac{\log ^{\beta}\left(r_{n}+g\left(r_{n}\right)+k\left(r_{n}\right)\right)}{\log ^{\beta}\left(r_{n}+(1-\xi) g\left(r_{n}\right)+k\left(r_{n}\right)\right)}\right)\left(1+\frac{r_{n}}{g\left(r_{n}\right)}+\frac{k\left(r_{n}\right)}{g\left(r_{n}\right)}\right)^{\alpha} \\
\Sigma_{n} & =c \frac{r_{n}^{\alpha}}{g\left(r_{n}\right)^{\alpha}}\left(1+\frac{k\left(r_{n}\right)}{r_{n}}\right)^{\alpha} \frac{\log ^{\beta}\left(r_{n}+k\left(r_{n}\right)\right)}{\log ^{\beta}\left(r_{n}+(1-\xi) g\left(r_{n}\right)+k\left(r_{n}\right)\right)} .
\end{aligned}
$$

Since $\Sigma_{n} \rightarrow 0$ as $n \rightarrow+\infty$, for every fixed $\varepsilon>0$ we can choose $n$ such that eventually $\Sigma_{n}<\varepsilon$. Moreover, since $\forall \xi \in(0,1)$

$$
\frac{\log ^{\beta}\left(r_{n}+g\left(r_{n}\right)+k\left(r_{n}\right)\right)}{\log ^{\beta}\left(r_{n}+(1-\xi) g\left(r_{n}\right)+k\left(r_{n}\right)\right)} \longrightarrow 1 \quad \text { as } n \rightarrow+\infty
$$

and using now $c>1$, we can choose a suitable $\xi$ such that $\Omega_{n}>2 \varepsilon$, if we choose $\varepsilon$ sufficiently small. Now letting $n \rightarrow+\infty$ we obtain that $\Theta_{n} \rightarrow+\infty$, which implies $g \notin \mathcal{B}(f, k, c)$, a contradiction that proves the lemma.

Note that the assumption $\alpha>0$ is necessary. It is not hard to see that, if $f(r)$ has polynomial growth, then $f$ does not satisfy property $(P)$ for any $c>0$.

Now, we are ready to prove our main technical result.

Theorem 6.5. Assume the validity of (V1 $\left.+\mathrm{V}_{\mathrm{L} 1}\right)$, (F2), (F3), (F4), (F5), (A2), (A3), (A4) and that $f$ satisfies property $(P)$ for the parameter $c>0$ required in (A4). Let $z \not \equiv 0$ be a locally Lipschitz solution of (5.18) on $\left[r_{0},+\infty\right)$. Let $\varrho \in\left[r_{0},+\infty\right)$, and let $R_{1}(\varrho), R_{2}(\varrho)$ be the first two consecutive zeros of $z(r)$ on $[\varrho,+\infty)$. Then

$$
R_{2}(\varrho)-\varrho=O(\varrho) \quad \text { as } \varrho \rightarrow+\infty .
$$

Moreover, in case $f(r)=\Lambda \exp \left\{a r^{\alpha} \log ^{\beta} r\right\}$ with $\Lambda, a, \alpha>0, \beta \geq 0$ we have the estimate

$$
\limsup _{\varrho \rightarrow+\infty} \frac{R_{2}(\varrho)}{\varrho} \leq\left(\frac{c+1}{c-1}\right)^{\frac{2}{\alpha}}
$$

for any $c>1$. let

Proof. As we have observed, $z(r)$ is oscillatory. Having fixed $\varrho \in\left[r_{0},+\infty\right)$,

$$
U=\left[\varrho, R_{2}(\varrho)\right) \backslash\left\{R_{1}(\varrho)\right\}
$$

and on $U$ consider the locally Lipschitz function

$$
y(r)=-\frac{v(r) z^{\prime}(r)}{z(r)}
$$

solution of

$$
y^{\prime}=A v+\frac{y^{2}}{v} \quad \text { a.e. on }\left[r_{0},+\infty\right)
$$

Because of (A2) and $\left(\mathrm{V} 1+\mathrm{V}_{\mathrm{L} 1}\right)$, by (6.22) $y$ is non-decreasing on $U$. In fact, from (A4), (F5), ( V1 $\left.+\mathrm{V}_{\mathrm{L} 1}\right)$ we can argue that $y$ is strictly increasing on $U$, so that

$$
y\left(R_{1}(\varrho)^{+}\right)=-\infty, \quad y\left(R_{1}(\varrho)^{-}\right)=+\infty, \quad y\left(R_{2}(\varrho)^{-}\right)=+\infty .
$$


To see this we only have to prove that $y$ cannot have finite limits. For instance, denote with $R$ a zero of $z$. If $y(r) \uparrow L<+\infty$ as $r \rightarrow R^{-}$,

$$
v(R) z^{\prime}(R)=\lim _{r \rightarrow R} v(r) z^{\prime}(r)=\lim _{r \rightarrow R} y(r) z(r)=0,
$$

therefore $z(r)$ should solve

$$
\left\{\begin{array}{l}
\left(v(r) z^{\prime}(r)\right)^{\prime}+A(r) v(r) z(r)=0 \quad \text { a.e. on } \mathbb{R}_{0}^{+} \\
z(R)=0, \quad v(R) z^{\prime}(R)=0 .
\end{array}\right.
$$

In other words, $z(r)$ should be a locally Lipschitz solution of Volterra integral problem

$$
z(r)=-\int_{R}^{r} \frac{1}{v(s)}\left\{\int_{R}^{s} A(x) v(x) z(x) \mathrm{d} x\right\} \mathrm{d} s=-\int_{R}^{r}\left[A(s) v(s) \int_{s}^{r} \frac{\mathrm{d} x}{v(x)}\right] z(s) \mathrm{d} s,
$$

where the last equality follows integrating by parts. Since $v(r)$ is bounded away from zero on compact sets of $\mathbb{R}^{+}$, the kernel of Volterra operator is locally bounded. Therefore, (6.26) has a unique local solution, which is necessarily $z \equiv 0$ on $\left[r_{0},+\infty\right)$. This contradicts $z \not \equiv 0$.

Since $y$ is increasing, $U$ can be decomposed as a disjoint union of intervals of the types

$$
\begin{array}{ll}
I_{1} \subseteq\{r \in U: y(r) \in[-1,1]\} & \text { interval of type } 1 \\
I_{2} \subseteq\{r \in U: y(r)>1\} & \text { interval of type } 2 \\
I_{3} \subseteq\{r \in U: y(r)<-1\} & \text { interval of type } 3
\end{array}
$$

To fix ideas we consider the case $y(\varrho)<-1$, which is "the worst" it could happen. The remaining cases can be dealt with similarly and we shall skip proofs. In this case we have

$$
U=I_{3} \cup I_{1} \cup I_{2} \cup I_{3}^{\prime} \cup I_{1}^{\prime} \cup I_{2}^{\prime}
$$

where, for each $i \in\{1,2,3\}$,

$$
I_{i} \text { is the first interval of type } i \text {, after } \varrho \text { and before } R_{1}(\varrho) \text {; }
$$

$I_{i}^{\prime}$ is the first interval of type $i$, after $R_{1}(\varrho)$ and before $R_{2}(\varrho)$.

For $i=\{1,2,3\}$ we set $\left|I_{i}\right|=g_{i}(\varrho)$ and $\left|I_{i}^{\prime}\right|=g_{i}^{\prime}(\varrho)$. We are going to prove that, in the above hypotheses, each $g_{i}(\varrho), g_{i}^{\prime}(\varrho)$ is $O(\varrho)$ as $\varrho \rightarrow+\infty$.

We consider at first an open interval $J$ of type 3 so that $J$ could be either $I_{3}$ or $I_{3}^{\prime}$. Set $P(\varrho)<Q(\varrho)$ to denote its end points; thus $g_{3}(\varrho)=|J|(\varrho)=Q(\varrho)-P(\varrho)$ and $g_{3}(\varrho)$ is clearly piecewise $C^{0}\left(\left[r_{0},+\infty\right)\right)$. We have $y(Q)=-1$ and $y(P) \leq-1$ if $y$ is defined in $P$, otherwise $y\left(P^{+}\right)=-\infty$. As in Theorem 5.1, (6.22) yields

$$
y^{\prime} \geq 2 \sqrt{A(r)}|y|=2 \sqrt{A(r)}(-y) \quad \text { a.e. on } J .
$$

Fix $r \in(P, Q]$ and integrate on $[r, Q]$. Recalling that $y(s) \leq y(Q)=-1 \forall s \in(P, Q]$ we have

$$
y(r) \leq-\exp \left\{2 \int_{r}^{Q} \sqrt{A(s)} \mathrm{d} s\right\} \quad \forall r \in(P, Q] .
$$

Since $y^{\prime} / y^{2} \geq 1 / v$, integrating on $[P+\varepsilon, r]$ for some small $\varepsilon>0$ we obtain

$$
\frac{1}{y(P+\varepsilon)}-\frac{1}{y(r)} \geq \int_{P+\varepsilon}^{r} \frac{\mathrm{d} s}{f(s)}
$$


and letting $\varepsilon \rightarrow 0^{+}$

$$
-\frac{1}{y(r)} \geq-\frac{1}{y\left(P^{+}\right)}+\int_{P}^{r} \frac{\mathrm{d} s}{f(s)} \geq \int_{P}^{r} \frac{\mathrm{d} s}{f(s)} \quad \forall r \in(P, Q] .
$$

Now, because of (A4)

$$
2 \int_{r}^{Q} \sqrt{A(s)} \mathrm{d} s \geq c \int_{r}^{Q} \frac{f^{\prime}(s)}{f(s)} \mathrm{d} s=\log \left(\frac{f(Q)}{f(r)}\right)^{c},
$$

and therefore, from (6.28),

$$
-\frac{1}{y(r)} \leq\left(\frac{f(r)}{f(Q)}\right)^{c} .
$$

Substituting into (6.30) and using (F3) we obtain

$$
1 \geq\left(\frac{f(Q)}{f(r)}\right)^{c} \int_{P}^{r} \frac{\mathrm{d} s}{f(s)} \geq(r-P) \frac{f(Q)^{c}}{f(r)^{c+1}} \quad \forall r \in(P, Q] .
$$

Suppose now that $J=I_{3}$, so that $P(\varrho)=\varrho$ and $Q(\varrho)=\varrho+g_{3}(\varrho)$. Since $r \in(P, Q)$, there exists $\xi \in(0,1)$ such that

$$
r=\varrho+(1-\xi) g_{3}(\varrho), \quad r-P=(1-\xi) g_{3}(\varrho)
$$

and since $r$ is arbitrary, from (6.31) we obtain

$$
\sup _{\xi \in(0,1)} \frac{(1-\xi) g_{3}(\varrho) f\left(\varrho+g_{3}(\varrho)\right)^{c}}{f\left(\varrho+(1-\xi) g_{3}(\varrho)\right)^{c+1}} \leq 1 .
$$

In this case, it follows that $g_{3} \in \mathcal{A}(f, 0, c)$ and then $g_{3}(\varrho)=O(\varrho)$ as $\varrho \rightarrow+\infty$. We will deal with the case $J=I_{3}^{\prime}$ later.

Next, we consider an interval $J$ of type 1 . Set $P(\varrho)<Q(\varrho)$ to denote its end points; thus $g_{1}(\varrho)=|J|(\varrho)=Q(\varrho)-P(\varrho)$ and $g_{1}(\varrho)$ is piecewise $C^{0}\left(\left[r_{0},+\infty\right)\right)$. In this case $y(P)=-1, y(Q)=1$ and $|y| \leq 1$ on $J$. We integrate Riccati equation $(6.22)$ on $[P, Q]$ to obtain

$$
2=\int_{P}^{Q} y^{\prime}(s) \mathrm{d} s=\int_{P}^{Q} A(s) v(s) \mathrm{d} s+\int_{P}^{Q} \frac{y^{2}(s)}{v(s)} \mathrm{d} s \geq \int_{P}^{Q} A(s) v(s) \mathrm{d} s .
$$

Now, without loss of generality we can suppose to have chosen $\varrho$ sufficiently large that $\left(\mathrm{V} 1+\mathrm{V}_{\mathrm{L} 1}\right)$, in particular $1 / v \in L^{1}(+\infty)$, implies

$$
\int_{\varrho}^{+\infty} \frac{\mathrm{d} s}{v(s)} \leq 1
$$

so that

$$
\int_{P}^{Q} \frac{\mathrm{d} s}{v(s)} \leq 1
$$

From (6.33), using (A4), the generalized mean value theorem and Holder inequality it follows that, for some $R_{0} \in[P, Q]$,

$$
\begin{aligned}
2 & \geq \int_{P}^{Q} A(s) v(s) \mathrm{d} s \int_{P}^{Q} \frac{\mathrm{d} s}{v(s)} \geq \int_{P}^{Q} \frac{c^{2}}{4}\left(\frac{f^{\prime}(s)}{f(s)}\right)^{2} v(s) \mathrm{d} s \int_{P}^{Q} \frac{\mathrm{d} s}{v(s)} \\
& =\frac{c^{2}}{4}\left(\frac{f^{\prime}\left(R_{0}\right)}{f\left(R_{0}\right)}\right)^{2} \int_{P}^{Q} v(s) \mathrm{d} s \int_{P}^{Q} \frac{\mathrm{d} s}{v(s)} \geq \frac{c^{2}}{4}\left(\frac{f^{\prime}\left(R_{0}\right)}{f\left(R_{0}\right)}\right)^{2}(Q-P)^{2},
\end{aligned}
$$


or, in other words, using (F2), (F3) and observing that (F5) implies that $f^{\prime}$ is eventually positive,

$$
\frac{2 \sqrt{2}}{c} \frac{f\left(R_{0}\right)}{f^{\prime}\left(R_{0}\right)} \geq Q-P .
$$

Now, if $J=I_{1}, P(\varrho)=\varrho+g_{3}(\varrho), Q(\varrho)=P(\varrho)+g_{1}(\varrho)$ and there exists $\theta \in[0,1]$ such that $R_{0}=\varrho+g_{3}(\varrho)+\theta g_{1}(\varrho)$. Substituting in (6.34) and using (F5) we obtain

$$
g_{1}(\varrho) \leq \frac{2 \sqrt{2}}{c} \frac{f\left(\varrho+g_{3}(\varrho)+\theta g_{1}(\varrho)\right)}{f^{\prime}\left(\varrho+g_{3}(\varrho)+\theta g_{1}(\varrho)\right)} \leq \frac{2 D \sqrt{2}}{c}\left(\varrho+g_{3}(\varrho)+\theta g_{1}(\varrho)\right)^{\mu} .
$$

If $\mu \leq 0$ we immediately obtain $g_{1}(\varrho)=O(\varrho)$. We turn our attention to the case $\mu \in(0,1)$. Using the already known equality $g_{3}(\varrho)=O(\varrho)$ and inequality $(x+y)^{\mu} \leq x^{\mu}+y^{\mu}$, there exist constants $K_{1}, K_{2}>0$ such that

$$
\frac{g_{1}(\varrho)}{\varrho} \leq \frac{K_{1}}{\varrho^{1-\mu}}+\frac{K_{2} g_{1}(\varrho)^{\mu}}{\varrho} .
$$

Using a simple reasoning by contradiction, (6.36) implies $g_{1}(\varrho)=O(\varrho)$ as $\varrho \rightarrow+\infty$.

If $J=I_{1}^{\prime}$,

$$
\begin{aligned}
P(\varrho) & =\varrho+\left(g_{1}+g_{2}+g_{3}\right)(\varrho)+g_{3}^{\prime}(\varrho), \\
Q(\varrho) & =P(\varrho)+g_{1}^{\prime}(\varrho), \\
R_{0} & =\varrho+\left(g_{1}+g_{2}+g_{3}\right)(\varrho)+g_{3}^{\prime}(\varrho)+\theta g_{1}^{\prime}(\varrho),
\end{aligned}
$$

and substituting into (6.34)

$$
g_{1}^{\prime}(\varrho) \leq \frac{2 \sqrt{2}}{c} \frac{f\left(\varrho+\left(g_{1}+g_{2}+g_{3}\right)(\varrho)+g_{3}^{\prime}(\varrho)+\theta g_{1}^{\prime}(\varrho)\right)}{f^{\prime}\left(\varrho+\left(g_{1}+g_{2}+g_{3}\right)(\varrho)+g_{3}^{\prime}(\varrho)+\theta g_{1}^{\prime}(\varrho)\right)}
$$

We will come back to this inequality later to prove $g_{1}^{\prime}(\varrho)=O(\varrho)$ as $\varrho \rightarrow+\infty$. Indeed, by the same argument as above, the only things that remain to show for this purpose are $g_{2}(\varrho)=O(\varrho)$ and $g_{3}^{\prime}(\varrho)=O(\varrho)$ as $\varrho \rightarrow+\infty$, and we are going to prove these facts now.

We consider an interval $J$ of type 2 and again let $P(\varrho)<Q(\varrho)$ denote its end points. Clearly $y(P)=1$ and $y(Q) \in(1,+\infty]$. Again

$$
y^{\prime} \geq 2 \sqrt{A(r)} y \quad \text { and } \quad \frac{y^{\prime}}{y^{2}} \geq \frac{1}{v} \quad \text { a.e. on } J .
$$

Fix $r \in[P, Q)$. Using $y(P)=1$, integration of the first inequality on $[P, r]$ yields

$$
y(r) \geq \exp \left\{2 \int_{P}^{r} \sqrt{A(s)} \mathrm{d} s\right\} \quad \forall r \in[P, Q),
$$

while integrating the second on $[r, Q-\varepsilon)$, for some small $\varepsilon>0$, and proceeding as in (6.29), we have

$$
\frac{1}{y(r)} \geq \int_{r}^{Q} \frac{\mathrm{d} s}{f(s)} \quad \forall r \in(P, Q) .
$$

Thus, observing that

$$
2 \int_{P}^{r} \sqrt{A(s)} \mathrm{d} s \geq \log \left(\frac{f(r)}{f(P)}\right)^{c}
$$


we deduce from (6.38)

$$
\frac{1}{y(r)} \leq\left(\frac{f(P)}{f(r)}\right)^{c}
$$

Finally, substituting into (6.39)

$$
1 \geq\left(\frac{f(r)}{f(P)}\right)^{c} \int_{r}^{Q} \frac{\mathrm{d} s}{f(s)} \geq(Q-r) \frac{1}{f(Q)}\left(\frac{f(r)}{f(P)}\right)^{c} \quad \forall r \in(P, Q) .
$$

Suppose now $J=I_{2}$ so that $g_{2}(\varrho)=Q(\varrho)-P(\varrho)$,

$$
\begin{aligned}
& P(\varrho)=\varrho+g_{3}(\varrho)+g_{1}(\varrho) ; \\
& Q(\varrho)=\varrho+g_{3}(\varrho)+g_{1}(\varrho)+g_{2}(\varrho),
\end{aligned}
$$

and since $r \in(P, Q)$, for some $\xi \in(0,1)$ we have

$$
\begin{aligned}
r & =\varrho+(1-\xi) g_{2}(\varrho)+g_{1}(\varrho)+g_{3}(\varrho) ; \\
Q-r & =\xi g_{2}(\varrho) .
\end{aligned}
$$

Substituting into (6.40) yields

$$
\sup _{\xi \in(0,1)} \frac{\xi g_{2}(\varrho) f\left(\varrho+(1-\xi) g_{2}(\varrho)+g_{1}(\varrho)+g_{3}(\varrho)\right)^{c}}{f\left(\varrho+g_{2}(\varrho)+g_{1}(\varrho)+g_{3}(\varrho)\right) f\left(\varrho+g_{1}(\varrho)+g_{3}(\varrho)\right)^{c}} \leq 1
$$

Thus, setting $\left(g_{1}+g_{3}\right)(\varrho)=k(\varrho)$ since $g_{1}(\varrho)=O(\varrho)$ and $g_{3}(\varrho)=O(\varrho)$ as $\varrho \rightarrow+\infty$, we have that $k(\varrho)=O(\varrho)$ as $\varrho \rightarrow+\infty$ and

$$
g_{2} \in \mathcal{B}(f, k, c),
$$

and so $g_{2}(\varrho)=O(\varrho)$ as $\varrho \rightarrow+\infty$.

We can now deal with the case $J=I_{3}^{\prime}$. We have already shown that $g_{1}(\varrho)+$ $g_{2}(\varrho)+g_{3}(\varrho)=O(\varrho)$ as $\varrho \rightarrow \infty$. We go back to $(6.31)$ with $J=I_{3}^{\prime}=(P(\varrho), Q(\varrho))$ : note that now

$$
P(\varrho)=\varrho+g_{3}(\varrho)+g_{1}(\varrho)+g_{2}(\varrho), \quad Q(\varrho)=P(\varrho)+g_{3}^{\prime}(\varrho) .
$$

Since $r \in(P, Q)$, for some $\xi \in(0,1)$ we have

$$
\begin{aligned}
r & =\varrho+(1-\xi) g_{3}^{\prime}(\varrho)+\left(g_{3}+g_{1}+g_{2}\right)(\varrho) ; \\
r-P & =(1-\xi) g_{3}^{\prime}(\varrho),
\end{aligned}
$$

and substituting into (6.31) we obtain

$$
\sup _{\xi \in(0,1)} \frac{(1-\xi) g_{3}^{\prime}(\varrho) f\left(\varrho+g_{3}^{\prime}(\varrho)+\left(g_{1}+g_{2}+g_{3}\right)(\varrho)\right)^{c}}{f\left(\varrho+(1-\xi) g_{3}^{\prime}(\varrho)+\left(g_{1}+g_{2}+g_{3}\right)(\varrho)\right)^{c+1}} \leq 1
$$

Thus, setting $h(\varrho)=\left(g_{1}+g_{2}+g_{3}\right)(\varrho), h(\varrho)=O(\varrho)$ as $\varrho \rightarrow+\infty$ and so we have $g_{3}^{\prime} \in \mathcal{A}(f, h, c)$ therefore $g_{3}^{\prime}(\varrho)=O(\varrho)$ as $\varrho \rightarrow+\infty$.

Coming back to inequality (6.37), we can now claim that $g_{1}^{\prime}(\varrho)=O(\varrho)$ as $\varrho \rightarrow+\infty$.

The last case is $J=I_{2}^{\prime}$ so that $g_{2}^{\prime}(\varrho)=Q(\varrho)-P(\varrho)$. Now we have

$$
\begin{aligned}
& P(\varrho)=\varrho+\left(g_{3}+g_{1}+g_{2}+g_{3}^{\prime}+g_{1}^{\prime}\right)(\varrho) \\
& Q(\varrho)=P(\varrho)+g_{2}^{\prime}(\varrho)
\end{aligned}
$$


and since $r \in(P, Q)$ there exists $\xi \in(0,1)$ such that

$$
\begin{aligned}
r & =\varrho+(1-\xi) g_{2}^{\prime}(\varrho)+\left(g_{3}+g_{1}+g_{2}+g_{3}^{\prime}+g_{1}^{\prime}\right)(\varrho) \\
Q(\varrho)-r & =\xi g_{2}^{\prime}(\varrho)
\end{aligned}
$$

Setting $k(\varrho)=\left(g_{3}+g_{1}+g_{2}+g_{3}^{\prime}+g_{1}^{\prime}\right)(\varrho)$, we have already proved that $k(\varrho)=O(\varrho)$ as $\varrho \rightarrow+\infty$. Substituting into (6.40) yields

$$
\sup _{\xi \in(0,1)} \frac{\xi g_{2}^{\prime}(\varrho) f\left(\varrho+(1-\xi) g_{2}^{\prime}(\varrho)+k(\varrho)\right)^{c}}{f\left(\varrho+g_{2}^{\prime}(\varrho)+k(\varrho)\right) f(\varrho+k(\varrho))^{c}} \leq 1
$$

Thus we have

$$
g_{2}^{\prime} \in \mathcal{B}(f, k, c),
$$

therefore $g_{2}^{\prime}(\varrho)=O(\varrho)$ as $\varrho \rightarrow+\infty$, and this shows that

$$
R_{2}(\varrho)-R_{1}(\varrho) \leq R_{2}(\varrho)-\varrho=\left(g_{3}+g_{1}+g_{2}+g_{3}^{\prime}+g_{1}^{\prime}+g_{2}^{\prime}\right)(\varrho)=O(\varrho)
$$

as $\varrho \rightarrow+\infty$, so we have proved the first part of the theorem, that is, (6.20).

To conclude, we shall estimate the quantity

$$
K=\limsup _{\varrho \rightarrow+\infty} \frac{R_{2}(\varrho)-\varrho}{\varrho} .
$$

Looking at the group of equations (6.32), (6.35), (6.41), (6.42), (6.37) and (6.43), we first note that each of the functions $g_{i}(\varrho)$ and $g_{i}^{\prime}(\varrho)$ involved in the proof, shortly $g(\varrho)$, satisfies one of the following inequalities, for $\varrho \geq r_{0}$ and for some suitable function $h(\varrho)$ which is known to be $O(\varrho)$ :

$$
\begin{array}{ll}
\sup _{\xi \in(0,1)} \frac{(1-\xi) g(\varrho) f(\varrho+g(\varrho)+h(\varrho))^{c}}{f(\varrho+(1-\xi) g(\varrho)+h(\varrho))^{c+1}} \leq 1 & \text { for } g_{3} \text { and } g_{3}^{\prime}, \\
g(\varrho) \leq \frac{2 \sqrt{2}}{c} \frac{f(\varrho+h(\varrho)+\theta g(\varrho))}{f^{\prime}(\varrho+h(\varrho)+\theta g(\varrho))} & \text { for } g_{1} \text { and } g_{1}^{\prime}, \\
\sup _{\xi \in(0,1)} \frac{\xi g(\varrho) f(\varrho+(1-\xi) g(\varrho)+h(\varrho))^{c}}{f(\varrho+g(\varrho)+h(\varrho)) \cdot f(\varrho+h(\varrho))^{c}} \leq 1 & \text { for } g_{2} \text { and } g_{2}^{\prime} .
\end{array}
$$

For the sake of simplicity, we perform computations in case

$$
f(r)=\Lambda \exp \left\{a r^{\alpha}\right\}, \quad a, \Lambda, \alpha>0 .
$$

We shall determine $K$ by computing, in each of the three cases above,

$$
K_{j}=\limsup _{\varrho \rightarrow+\infty} \frac{g(\varrho)}{\varrho}
$$

(the index $j$ corresponds to the cases satisfied by $g_{j}$ and $g_{j}^{\prime}$ ), and then summing the terms "inductively" following the changes of the known function $h$ case by case. For this purpose let

$$
H \geq \limsup _{\varrho \rightarrow+\infty} \frac{h(\varrho)}{\varrho}
$$


Consider at first inequality (6.45): we immediately find that, for this choice of f,

$$
\frac{g(\varrho)}{\varrho} \leq \frac{2 \sqrt{2}}{c} \frac{1}{\varrho} \frac{1}{a \alpha(\varrho+h(\varrho)+\theta g(\varrho))^{\alpha-1}} \leq \frac{2 \sqrt{2}}{c a \alpha} \frac{1}{\varrho^{\alpha}}\left(1+\frac{h(\varrho)}{\varrho}+\frac{g(\varrho)}{\varrho}\right)^{1-\alpha}
$$

We claim that $K_{1}=0$. Indeed, suppose by contradiction that there exists a divergent sequence $\left\{\varrho_{n}\right\}$ such that $g\left(\varrho_{n}\right) / \varrho_{n} \rightarrow K_{1}>0$. Then, evaluating the above inequality along $\left\{\varrho_{n}\right\}$ and passing to the limit we obtain $0<K_{1} \leq 0$, a contradiction. We now focus our attention on (6.44). By an algebraic manipulation

$$
g(\varrho) \leq \frac{1}{1-\xi} \frac{f(\varrho+(1-\xi) g(\varrho)+h(\varrho))^{c+1}}{f(\varrho+g(\varrho)+h(\varrho))^{c}} \quad \forall \xi \in(0,1) .
$$

Due to the form of $f$, better estimates can be obtained by choosing $\xi$ near 1 . For $\varrho>1$, we choose $\xi=(\varrho-1) / \varrho$. For the ease of notation let $x(\varrho)=g(\varrho) / \varrho$, so that $x(\varrho)$ is bounded on $\left[r_{0},+\infty\right)$ because $f$ satisfies property $(P)$. With this choice of $\xi$ we have

$$
x(\varrho) \leq \frac{f(\varrho+x(\varrho)+h(\varrho))^{c+1}}{f(\varrho+\varrho x(\varrho)+h(\varrho))^{c}}
$$

thus substituting

$$
x(\varrho) \leq \Lambda \exp \left\{a \varrho^{\alpha}\left[(c+1)\left(1+\frac{x(\varrho)}{\varrho}+\frac{h(\varrho)}{\varrho}\right)^{\alpha}-c\left(1+x(\varrho)+\frac{h(\varrho)}{\varrho}\right)^{\alpha}\right]\right\} .
$$

Suppose now that $K_{3}>0$, and evaluate this inequality along a sequence $\left\{\varrho_{n}\right\}$ such that $x\left(\varrho_{n}\right) \rightarrow K_{3}$. Choose $0<\delta<K_{3}$, and let $n$ be large enough that the following inequalities hold:

This yields:

$$
x\left(\varrho_{n}\right)>K_{3}-\delta, \quad \frac{x\left(\varrho_{n}\right)}{\varrho_{n}}<\delta
$$

$$
x\left(\varrho_{n}\right) \leq \Lambda \exp \left\{a \varrho_{n}^{\alpha}\left[(c+1)\left(1+\delta+\frac{h\left(\varrho_{n}\right)}{\varrho_{n}}\right)^{\alpha}-c\left(1+K_{3}-\delta+\frac{h\left(\varrho_{n}\right)}{\varrho_{n}}\right)^{\alpha}\right]\right\} .
$$

Suppose now that $K_{3}$ satisfies

$$
\max _{\mu \in[0, H]}\left\{(c+1)(1+\mu)^{\alpha}-c\left(1+K_{3}+\mu\right)^{\alpha}\right\}<0
$$

and compare it with (6.48). We can say, by continuity, that there exists a small $\delta>0$ such that the expression between square brackets is strictly less than 0 . Letting now $\varrho_{n}$ go to infinity in (6.48) we deduce $0<K_{3} \leq 0$, a contradiction. Note that (6.49) holds if and only if

$$
(c+1)-c\left(\frac{K_{3}}{\mu+1}+1\right)^{\alpha}<0 \quad \forall \mu \in[0, H],
$$

that is,

$$
K_{3}>\left[\left(\frac{c+1}{c}\right)^{\frac{1}{\alpha}}-1\right](1+H) .
$$

Hence, if $K_{3}>0$, we necessarily have

$$
K_{3} \leq\left[\left(\frac{c+1}{c}\right)^{\frac{1}{\alpha}}-1\right](1+H) .
$$


The same technique can be exploited when dealing with (6.46): from

$$
g(\varrho) \leq \frac{1}{\xi} \frac{f(\varrho+g(\varrho)+h(\varrho)) \cdot f(\varrho+h(\varrho))^{c}}{f(\varrho+(1-\xi) g(\varrho)+h(\varrho))^{c}} \quad \forall \xi \in(0,1)
$$

we deduce that it is better to choose $\xi$ near 0 , so we set $\xi=1 / \varrho$ and, with the same notations above, we obtain

$$
x(\varrho) \leq \frac{f(\varrho+\varrho x(\varrho)+h(\varrho)) \cdot f(\varrho+h(\varrho))^{c}}{f(\varrho+(\varrho-1) x(\varrho)+h(\varrho))^{c}} .
$$

Thus,

$$
\begin{aligned}
x(\varrho) \leq & \Lambda \exp \left\{a \varrho ^ { \alpha } \left[\left(1+x(\varrho)+\frac{h(\varrho)}{\varrho}\right)^{\alpha}+\right.\right. \\
& \left.\left.+c\left(1+\frac{h(\varrho)}{\varrho}\right)^{\alpha}-c\left(1+\frac{\varrho-1}{\varrho} x(\varrho)+\frac{h(\varrho)}{\varrho}\right)^{\alpha}\right]\right\} .
\end{aligned}
$$

Next, if $K_{2}>0$ we choose a sequence $\left\{\varrho_{n}\right\}$ realizing $K_{2}$ and we consider $n$ sufficiently large that

$$
\frac{\left(\varrho_{n}-1\right)}{\varrho_{n}}>(1-\delta), \quad K_{2}-\delta<x\left(\varrho_{n}\right)<K_{2}+\delta
$$

obtaining the estimate

$$
\begin{aligned}
x\left(\varrho_{n}\right) \leq & \Lambda \exp \left\{a \varrho _ { n } ^ { \alpha } \cdot \left[\left(1+\left(K_{2}+\delta\right)+\frac{h\left(\varrho_{n}\right)}{\varrho_{n}}\right)^{\alpha}+\right.\right. \\
& \left.\left.+c\left(1+\frac{h\left(\varrho_{n}\right)}{\varrho_{n}}\right)^{\alpha}-c\left(1+(1-\delta)\left(K_{2}-\delta\right)+\frac{h\left(\varrho_{n}\right)}{\varrho_{n}}\right)^{\alpha}\right]\right\}
\end{aligned}
$$

Now, if $K_{2}$ satisfies

$$
\max _{\mu \in[0, H]}\left\{\left(1+K_{2}+\mu\right)^{\alpha}+c(1+\mu)^{\alpha}-c\left(1+K_{2}+\mu\right)^{\alpha}\right\}<0
$$

we reach a contradiction proceeding as in the previous case. Similarly to what we did above this yields the bound

$$
K_{2} \leq\left[\left(\frac{c}{c-1}\right)^{\frac{1}{\alpha}}-1\right](1+H) .
$$

To simplify the writing we now set

$$
W=\left[\left(\frac{c+1}{c}\right)^{\frac{1}{\alpha}}-1\right], \quad Z=\left[\left(\frac{c}{c-1}\right)^{\frac{1}{\alpha}}-1\right] .
$$

To estimate $g_{3}(\varrho) / \varrho$, we shall use (6.50) and, from (6.32), we deduce $h(\varrho) \equiv 0$ and thus $H=0$. Therefore, we get

$$
K_{3} \leq W
$$

We have already shown that $K_{1}=0$. Next, to estimate $g_{2}(\varrho) / \varrho$ we shall consider (6.54). By $(6.41) h(\varrho)=g_{3}(\varrho)+g_{1}(\varrho)$, so we can use for $H$ the sum $W+0=W$, hence

$$
K_{2} \leq Z(1+W)
$$


Proceeding along the same lines we obtain the estimates

$$
\begin{aligned}
& K_{3}^{\prime} \leq W(1+W+Z(1+W)) ; \\
& K_{1}^{\prime}=0 ; \\
& K_{2}^{\prime} \leq Z(1+W+Z(1+W)+W(1+W+Z(1+W))) .
\end{aligned}
$$

Summing up the $K_{j}$ and the $K_{j}^{\prime}$, we obtain the surprisingly simple expression

$$
K \leq \sum_{j=1}^{3}\left(K_{j}+K_{j}^{\prime}\right)=(W+1)^{2}(Z+1)^{2}-1=\left(\frac{c+1}{c-1}\right)^{\frac{2}{\alpha}}-1,
$$

therefore the upper estimate (6.21) holds true. With few modifications it can be seen that, adding the logarithmic term in the definition of $f$, the value of $K$ does not change.

REMARK 6.6. One might ask if, varying the choice of the level sets in (6.27), one could obtain better estimates. It is not hard to see that, for every choice of fixed level sets, (6.21) does not change.

The discussion at the beginning of this section motivates the following

\section{Question:}

(4) Is it true that, in the assumptions of Theorem 6.5,

$$
\lim _{\varrho \rightarrow+\infty} \frac{R_{2}(\varrho)}{\varrho}=1 ?
$$

\subsection{The growth of the index of $-\Delta-q(x)$}

As an immediate example, we quote the following estimate for the growth of the index of Schrödinger operators.

THEOREM 6.7. Let $(M,\langle\rangle$,$) be an m-dimensional complete Riemannian man-$ ifold such that

$$
\left(\operatorname{vol}\left(\partial B_{r}\right)\right)^{-1} \in L^{1}(+\infty), \quad \operatorname{vol}\left(\partial B_{r}\right) \leq \Lambda \exp \left\{a r^{\alpha} \log ^{\beta} r\right\},
$$

for some $\Lambda, a, \alpha>0, \beta \geq 0$. Let $q(x) \in L_{\mathrm{loc}}^{\infty}(M)$, and let $\bar{q}(r)$ be its spherical mean. Assume that, for some $r_{0}>0$ and $c>1$,

$$
\bar{q}(r) \geq c^{2}\left(\frac{a \alpha}{2}\right)^{2} r^{2(\alpha-1)} \log ^{2 \beta} r \quad \forall r \geq r_{0}
$$

Then, $L=-\Delta-q(x)$ has infinite index and

$$
\liminf _{r \rightarrow+\infty} \frac{\operatorname{ind}_{L}\left(B_{r}\right)}{\log r} \geq \frac{\alpha}{2 \log \left(\frac{c+1}{c-1}\right)} .
$$

ProOF. In our assumptions (6.55), (6.56), by Theorem 6.5 and the previous observations $\left(v z^{\prime}\right)^{\prime}+A v z=0$ with $A(r)=\bar{q}(r)$ is oscillatory, thus $L$ has infinite index by the same technique as in Theorem 5.15. Note that (6.57) is equivalent to proving that

$$
\liminf _{r \rightarrow+\infty} \frac{\operatorname{ind}_{L}\left(B_{r}\right)}{\log r} \geq \frac{1}{\log \mu}, \quad \text { where } \quad \mu=\left(\frac{c+1}{c-1}\right)^{\frac{2}{\alpha}} .
$$


Fix $\varepsilon>0$. Then, by Theorem 6.5 there exists $R=R(\varepsilon)$ such that on $[R,+\infty)$

$$
\frac{R_{2}(r)}{r}<\mu_{\varepsilon}=\left(\frac{c+1}{c-1}\right)^{\frac{2}{\alpha}}+\varepsilon
$$

Proceeding as in Theorem 5.15, on $M \backslash B_{r}$ we can find a radial function $\psi_{1}(x)$, with support strictly inside $B_{\mu_{\varepsilon} r}$, whose Rayleigh quotient is zero, hence $\lambda_{1}^{L}\left(B_{\mu_{\varepsilon} r}\right)<0$. Starting from $R_{2}(r)$, the second zero after $R_{2}(r)$ is attained before $\mu_{\varepsilon} R_{2}(r)<\mu_{\varepsilon}^{2} r$, and we can construct a new Lipschitz radial function $\psi_{2}(x)$ whose Rayleigh quotient is zero. Moreover, the supports of $\psi_{2}$ and $\psi_{1}$ are disjoint. In conclusion, the index of $L$ grows at least by 1 when the radius is multiplied by $\mu_{\varepsilon}$, hence

$$
\operatorname{ind}_{L}\left(B_{r}\right) \geq \operatorname{ind}_{L}\left(B_{R}\right)+\left\lfloor\log _{\mu_{\varepsilon}}\left(\frac{r}{R}\right)\right\rfloor
$$

where $\lfloor s\rfloor$ denotes the floor of $s$. Therefore we have

$$
\liminf _{r \rightarrow+\infty} \frac{\operatorname{ind}_{L}\left(B_{r}\right)}{\log _{\mu_{\varepsilon}} r} \geq 1 \quad \forall \varepsilon>0 .
$$

Changing the base of the logarithm yields

$$
\liminf _{r \rightarrow+\infty} \frac{\operatorname{ind}_{L}\left(B_{r}\right)}{\log r} \geq \frac{1}{\log \mu_{\varepsilon}} \quad \forall \varepsilon>0,
$$

and letting $\varepsilon \rightarrow 0$ gives the desired conclusion.

\subsection{The essential spectrum of $-\Delta$ and punctured manifolds}

Our purpose here is to apply oscillation estimates to find sharp bounds for the spectral radius of $M \backslash B_{R}$ as a function of $R$, when the volume growth is faster than exponential. To see which kind of bound we should expect, we readapt Do Carmo and Zhou example 2.24. Let $\left(M_{g}, \mathrm{~d} s^{2}\right)$ be a model manifold with

$$
g(r)= \begin{cases}r & \text { on }[0,1] ; \\ \exp \left\{\frac{a r^{\alpha}}{m-1}\right\} & \text { on }[2,+\infty),\end{cases}
$$

for some $a>0, \alpha \geq 1$. Note that, for $r \geq 2, \operatorname{vol}\left(\partial B_{r}\right)=\exp \left\{a r^{\alpha}\right\}$. We let $b \in(0, a)$ and set

$$
u_{b}(x)=\exp \left\{-b r(x)^{\alpha}\right\} \quad \text { on } M \backslash B_{2} .
$$

A simple checking shows that

$$
\Delta u_{b}+\lambda_{b}(r) u_{b}=0 \quad \text { on } M \backslash B_{2},
$$

where $\lambda_{b}(r)$ is defined as

$$
\lambda_{b}(r)=\alpha^{2} b(a-b) r^{2(\alpha-1)}+\alpha(\alpha-1) b r^{\alpha-2} .
$$

Observe that, in case $\alpha=1, \lambda_{b}(r) \equiv b(a-b)$, while, if $\alpha>1, \lambda_{b}(r)$ is strictly increasing on $\left(r_{0},+\infty\right)$, with $r_{0}$ sufficiently large that

$$
2 \alpha(a-b) r_{0}^{\alpha}+(\alpha-2)>0 .
$$

Up to further enlarging $r_{0}$, we can also assume that

$$
\frac{\alpha-1}{2 \alpha} \frac{1}{r^{\alpha}}<\frac{a}{2} \quad \text { for } r \geq r_{0} .
$$


Barta theorem [Bar37] gives, for every $b \in(0, a), R \geq r_{0}$,

$$
\lambda_{1}^{-\Delta}\left(M \backslash B_{R}\right) \geq \inf _{M \backslash B_{R}}\left(-\frac{\Delta u_{b}}{u_{b}}\right)=\inf _{[R,+\infty)} \lambda_{b}(r)=\lambda_{b}(R) .
$$

The choice

$$
\widetilde{b}=\frac{a}{2}+\frac{\alpha-1}{2 \alpha} \frac{1}{R^{\alpha}}
$$

maximize $\lambda_{b}(R)$ and $\widetilde{b} \in(0, a)$ because of (6.63). Then, for $R \geq r_{0}$

$$
\lambda_{1}^{-\Delta}\left(M \backslash B_{R}\right) \geq \alpha^{2}\left(\frac{a^{2}}{4}-\frac{(\alpha-1)^{2}}{4 \alpha^{2}} \frac{1}{R^{2 \alpha}}\right) R^{2(\alpha-1)}
$$

so that

$$
\liminf _{R \rightarrow+\infty}\left(\frac{\lambda_{1}^{-\Delta}\left(M \backslash B_{R}\right)}{R^{2(\alpha-1)}}\right) \geq \frac{a^{2} \alpha^{2}}{4} .
$$

Note that for $\alpha=1$ the above reduces to

$$
\lambda_{1}^{-\Delta}\left(M \backslash B_{R}\right) \geq \frac{a^{2}}{4} \quad \text { for every } R \geq r_{0},
$$

coherently with Theorem 2.23. This example, for $\operatorname{vol}\left(\partial B_{r}\right) \leq C \exp \left\{a r^{\alpha}\right\}, C, a>0$, $\alpha \geq 1$, suggests to look for an upper bound of $\lambda_{1}^{-\Delta}\left(M \backslash B_{R}\right)$ of the form

$$
C_{1} R^{2(\alpha-1)}
$$

with $C_{1}=C_{1}(a, \alpha)>0$. The guess is indeed correct:

THEOREM 6.8. If $M$ is a complete, non-compact Riemannian manifold such that

$$
\left(\operatorname{vol}\left(\partial B_{r}\right)\right)^{-1} \in L^{1}(+\infty), \quad \operatorname{vol}\left(\partial B_{r}\right) \leq \Lambda \exp \left\{a r^{\alpha} \log ^{\beta} r\right\}
$$

for $r$ large and for some $\Lambda, a, \alpha>0, \beta \geq 0$, the following estimates hold:

- If $0<\alpha<1$ then

$$
\lambda_{1}^{-\Delta}\left(M \backslash B_{R}\right)=0 \quad \forall R \geq 0 .
$$

- If $\alpha=1, \beta=0$ then

$$
\lambda_{1}^{-\Delta}\left(M \backslash B_{R}\right) \leq \frac{a^{2}}{4} \quad \forall R \geq 0 .
$$

- If $\alpha \geq 1$ then

$$
\limsup _{R \rightarrow+\infty}\left(\frac{\lambda_{1}^{-\Delta}\left(M \backslash B_{R}\right)}{R^{2(\alpha-1)} \log ^{2 \beta} R}\right) \leq \frac{a^{2} \alpha^{2}}{4} \inf _{c \in(1,+\infty)}\left\{c^{2}\left(\frac{c+1}{c-1}\right)^{\frac{4(\alpha-1)}{\alpha}}\right\} .
$$

Remark 6.9. Note that $\left(\operatorname{vol}\left(\partial B_{r}\right)\right)^{-1} \in L^{1}(+\infty)$ implies $\operatorname{vol}(M)=\infty$ from Schwarz inequality

$$
\int_{r}^{R} \frac{\mathrm{d} s}{\operatorname{vol}\left(\partial B_{s}\right)} \int_{r}^{R} \operatorname{vol}\left(\partial B_{s}\right) \mathrm{d} s \geq(R-r)^{2}
$$

letting $R \rightarrow+\infty$. Therefore, the cases $\alpha \in(0,1)$ and $\alpha=1, \beta=0$ already follow from Taylor-Brooks-Higuchi Theorem 2.23 (see also [CZ99]). We have decided to add them to the statement of Theorem 6.8 since they can be easily proved with our techniques. 
We stress that, while the hypothesis $\operatorname{vol}(M)=\infty$ is essential as already explained in Remark 2.24, the stronger assumption $\left(\operatorname{vol}\left(\partial B_{r}\right)\right)^{-1} \in L^{1}(+\infty)$ is for convenience: if it fails, we will show in the next lemma that $\lambda_{1}^{\Delta}\left(M \backslash B_{R}\right)=0$ for every $R \geq 0$.

Lemma 6.10. Let $(M,\langle\rangle$,$) be a complete Riemannian manifold satisfying$

$$
\operatorname{vol}\left(\partial B_{r}\right) \leq f(r) \quad \text { on }\left(r_{0},+\infty\right)
$$

for some $r_{0}$ sufficiently large and some $f \in C^{0}\left(\left[r_{0},+\infty\right)\right)$. Fix $R \geq 0$.

- If $M$ has infinite volume and $\left(\operatorname{vol}\left(\partial B_{r}\right)\right)^{-1} \notin L^{1}(+\infty)$ then

$$
\lambda_{1}^{-\Delta}\left(M \backslash B_{R}\right)=0 .
$$

- If $\left(\operatorname{vol}\left(\partial B_{r}\right)\right)^{-1} \in L^{1}(+\infty)$, then for every $\varepsilon>0$ there exists $r_{1}=r_{1}(\varepsilon)>$ $R$ such that

$$
\lambda_{1}^{-\Delta}\left(M \backslash B_{R}\right) \leq\left\{\inf _{r>r_{1}}\left[-\frac{1}{2} \frac{\log \int_{r}^{+\infty} \frac{\mathrm{d} s}{f(s)}}{r-r_{1}}\right]\right\}^{2}+\varepsilon
$$

Proof. Set $v(r)=\operatorname{vol}\left(\partial B_{r}\right)$. We begin with the case $1 / v \in L^{1}(+\infty)$. Up to further enlarging $r_{0}$, we can assume that

$$
r_{0}>R, \quad \int_{r_{0}}^{+\infty} \frac{\mathrm{d} s}{v(s)}<1
$$

and let $\varepsilon>0$. We define on $\left[r_{0},+\infty\right)$

$$
A_{\varepsilon}(r)=\left\{\inf _{s>r}\left[-\frac{1}{2} \frac{\log \int_{s}^{+\infty} \frac{\mathrm{d} x}{f(x)}}{s-r}\right]\right\}^{2}+\varepsilon
$$

Then, $A_{\varepsilon}(r) \geq \varepsilon, A_{\varepsilon}(r)$ is continuous and non-decreasing. By Remark 6.9, $M$ has infinite volume, thus we can apply $(v)$ of Proposition 5.9 to obtain that

$$
\left(v(r) z_{\varepsilon}^{\prime}(r)\right)^{\prime}+A_{\varepsilon}(r) v(r) z_{\varepsilon}(r)=0
$$

is oscillatory. Let $\varrho_{1}<\varrho_{2}$ be two consecutive zeroes of $z_{\varepsilon}$ after $r_{0}$. Define $\phi(x)=$ $z_{\varepsilon}(r(x))$ on $B_{\varrho_{2}} \backslash B_{\varrho_{1}}$. By the domain monotonicity of eigenvalues and integrating by parts we have

$$
\begin{aligned}
0 \leq \lambda_{1}^{-\Delta}\left(M \backslash B_{R}\right)<\lambda_{1}^{-\Delta}\left(B_{\varrho_{2}} \backslash B_{\varrho_{1}}\right) & \leq \frac{\int_{B_{\varrho_{2}} \backslash B_{\varrho_{1}}}|\nabla \phi|^{2}}{\int_{B_{\varrho_{2}} \backslash B_{\varrho_{1}}} \phi^{2}}=\frac{\int_{\varrho_{1}}^{\varrho_{2}}\left[z_{\varepsilon}^{\prime}(s)\right]^{2} v(s) \mathrm{d} s}{\int_{\varrho_{1}}^{\varrho_{2}} z_{\varepsilon}(s)^{2} v(s) \mathrm{d} s} \\
& =\frac{\int_{\varrho_{1}}^{\varrho_{2}} A_{\varepsilon}(s) z_{\varepsilon}(s)^{2} v(s) \mathrm{d} s}{\int_{\varrho_{1}}^{\varrho_{2}} z_{\varepsilon}(s)^{2} v(s) \mathrm{d} s} \leq A_{\varepsilon}\left(\varrho_{2}\right)
\end{aligned}
$$

Thus we get (6.67) with $r_{1}=\varrho_{2}$ (note that $r_{1}$ depends on $\varepsilon$ since $z_{\varepsilon}(r)$ does).

In case $1 / v \notin L^{1}(+\infty)$ and $M$ has infinite volume, by Theorem 5.6 equation $\left(v z^{\prime}\right)^{\prime}+A v z=0$ is oscillatory whenever $A(r) \geq \varepsilon>0$ : indeed

$$
\int_{r_{0}}^{+\infty} A(s) v(s) \mathrm{d} s \geq \varepsilon \int_{r_{0}}^{+\infty} v(s) \mathrm{d} s=+\infty .
$$

Choosing $A_{\varepsilon}(r)=\varepsilon$ and using the Rayleigh quotient as before we deduce (6.66) at once. 
Lemma 6.11. If $1 / v \in L^{1}(+\infty)$, the previous lemma yields in particular the weaker estimate

$$
\lambda_{1}^{-\Delta}\left(M \backslash B_{R}\right) \leq\left\{\liminf _{r \rightarrow+\infty}\left[-\frac{1}{2} \frac{\log \int_{r}^{+\infty} \frac{\mathrm{d} s}{f(s)}}{r}\right]\right\}^{2} \quad \forall R \geq 0 .
$$

Proof. This follows immediately from the next observation: if we substitute in (6.67) "inf" with the greater "liminf", the latter does not depend on $R_{0}(\varepsilon)$. We can thus fix a particular $R_{0}(\varepsilon)$, compute the "liminf" and then let $\varepsilon \rightarrow 0$.

We are now ready to prove Theorem 6.8.

Proof. First, we apply Lemma 6.11 to estimate $\lambda_{1}^{-\Delta}\left(M \backslash B_{R}\right)$ when the volume growth is at most exponential. Towards this aim suppose that $\left(\operatorname{vol}\left(\partial B_{r}\right)\right)^{-1} \in$ $L^{1}(+\infty)$ and that

$$
\operatorname{vol}\left(\partial B_{r}\right) \leq f(r)=\Lambda \exp \left\{a r^{\alpha}\right\} \quad 0<\alpha \leq 1, \quad \Lambda, a>0 .
$$

Due to our choice of $\alpha$ we easily see that

$$
-\frac{1}{2} \frac{\log \int_{r}^{+\infty} \frac{\mathrm{d} s}{f(s)}}{r} \sim \frac{a}{2} r^{\alpha-1} \quad \text { as } r \rightarrow+\infty .
$$

Because of this we can apply Lemma 6.11 to deduce that, for every $R \geq 0$,

$$
\lambda_{1}^{-\Delta}\left(M \backslash B_{R}\right) \leq \begin{cases}0 & \text { if } 0<\alpha<1 ; \\ a^{2} / 4 & \text { if } \alpha=1 .\end{cases}
$$

The above works also when $\operatorname{vol}\left(\partial B_{r}\right) \leq \Lambda \exp \left\{a r^{\alpha} \log ^{\beta} r\right\}$, with $\alpha<1, \beta \geq 0$, since it is enough to observe that

$$
\exp \left\{a r^{\alpha} \log ^{\beta} r\right\}=O\left(\exp \left\{a r^{\widetilde{\alpha}}\right\}\right) \quad \text { for every } 1>\widetilde{\alpha}>\alpha .
$$

We are left with the case $\alpha \geq 1, \beta \geq 0$. For $c>1$ and $r>R$ we define

$$
A(r)=\left[c\left(\frac{a \alpha}{2}\right) r^{\alpha-1} \log ^{\beta} r\right]^{2} .
$$

Note that $A(r)$ is monotone non-decreasing and, by Theorem 6.5 and the previous observations $\left(v z^{\prime}\right)^{\prime}+A v z=0$ is oscillatory. Hence, proceeding as in Lemma 6.10 we have for $R \geq r_{0}$

$$
\lambda_{1}^{-\Delta}\left(M \backslash B_{R}\right) \leq A\left(\varrho_{2}\right),
$$

where $\varrho_{2}(R)$ is the second zero of the solution $z$ of (5.18) after $R$. By Theorem 6.5, for every $\varepsilon>0$ there exists $r_{1}(\varepsilon)$ such that, for every $R \geq r_{1}$

$$
\rho_{2}(R) \leq\left[\left(\frac{c+1}{c-1}\right)^{\frac{2}{\alpha}}(1+\varepsilon)\right] R
$$

Therefore, from the monotonicity of $A(r)$ we get

$$
\lambda_{1}^{-\Delta}\left(M \backslash B_{R}\right) \leq A\left(\left[\left(\frac{c+1}{c-1}\right)^{\frac{2}{\alpha}}(1+\varepsilon)\right] R\right) \quad \forall R \geq r_{1}(\varepsilon) .
$$


Using the definition of $A(r)$, up to choosing $\varepsilon$ small enough and $r_{2} \geq r_{1}$ large enough we deduce that, for every fixed $c>1$,

$\lambda_{1}^{-\Delta}\left(M \backslash B_{R}\right) \leq \frac{a^{2} \alpha^{2}}{4} R^{2(\alpha-1)} \log ^{2 \beta} R\left[c^{2}\left(\frac{c+1}{c-1}\right)^{\frac{4(\alpha-1)}{\alpha}}\right](1+2 \varepsilon) \quad \forall R \geq r_{2}(\varepsilon)$.

Thus, letting first $R \rightarrow+\infty$ and then $\varepsilon \rightarrow 0$, and minimizing over all $c \in(1,+\infty)$ we finally have

$$
\limsup _{R \rightarrow+\infty}\left(\frac{\lambda_{1}^{-\Delta}\left(M \backslash B_{R}\right)}{R^{2(\alpha-1)} \log ^{2 \beta} R}\right) \leq \frac{a^{2} \alpha^{2}}{4} \inf _{c \in(1,+\infty)}\left\{c^{2}\left(\frac{c+1}{c-1}\right)^{\frac{4(\alpha-1)}{\alpha}}\right\},
$$

as desired.

REMARK 6.12. The infimum of the function

$$
c^{2}\left(\frac{c+1}{c-1}\right)^{\frac{4(\alpha-1)}{\alpha}}
$$

is attained by the unique positive solution $c$ of $\alpha(c+1)(c-1)=4(\alpha-1) c$, which can be computed, although its explicit expression is not so neat.

REMARK 6.13. It is worth to point out that estimate (6.71) fits with the estimate (6.70) for $\alpha=1$ and $\beta=0$.

REMARK 6.14. As in the introduction of this section, one can study a model manifold whose function $g(r)$ is of the following type:

$$
g(r)= \begin{cases}r & r \in[0,1] \\ \exp \left\{\frac{a r^{\alpha}}{m-1} \log ^{\beta} r\right\} & r \in[2,+\infty),\end{cases}
$$

for which the volume growth of geodesic spheres is

$$
\exp \left\{a r^{\alpha} \log ^{\beta} r\right\} \text {. }
$$

With the same computations, one obtains for $R$ sufficiently large

$$
\lambda_{1}^{-\Delta}\left(M \backslash B_{R}\right) \geq C R^{2(\alpha-1)} \log ^{2 \beta} R,
$$

for some $C>0$. This shows that the estimate of Theorem 6.8 is sharp even with respect to the power of the logarithm.

We briefly describe an interesting application, due to M.P. Do Carmo and D. Zhou in [CZ99], of spectral estimates to constant mean curvature hypersurfaces. Let $\varphi: M^{m} \rightarrow N^{m+1}$ be a CMC, orientable hypersurface into an orientable ambient manifold $N$. Let $\nu$ be a chosen orientation of $M$. We refer to Section 5.4 both for notations and basic background. The Jacobi operator associated to the stability of $M$ is

$$
L=-\Delta-\left(|I I|^{2}+\overline{\operatorname{Ricc}}(\nu, \nu)\right),
$$

And $M$ is called stable, respectively of finite index, if so is $L$. 
Proposition 6.15 ([CZ99], Theorem 4.2). Let $\varphi: M^{m} \rightarrow N^{m+1}$ be a CMC hypersurface with $\operatorname{vol}(M)=+\infty$ into an oriented, complete Riemannian manifold. Assume that $M$ has finite stability index, and that

$$
\liminf _{r \rightarrow+\infty} \frac{\log \operatorname{vol}\left(B_{r}\right)}{r}=a<+\infty
$$

Then,

$$
H^{2} \leq \frac{1}{m}\left(\frac{a^{2}}{4}-\liminf _{x \rightarrow \infty} \overline{\operatorname{Ricc}}(\nu, \nu)\right)
$$

In particular, if $M$ has subexponential growth and $\overline{\text { Ricc }} \geq 0$, then $M$ is minimal.

Proof. By Theorem 1.41, there exists $r_{0}>0$ and a smooth $w>0$ on $M \backslash \bar{B}_{r_{0}}$ satisfying $L w=0$. Then, By Theorem 2.23 and Persson formula (1.88), for every $R>r_{0}$

$$
\begin{aligned}
\frac{a^{2}}{4} & \geq \lambda_{1}^{-\Delta}\left(M \backslash B_{R}\right) \geq-\inf _{M \backslash B_{R}} \frac{\Delta w}{w}=\inf _{M \backslash B_{R}}\left(|I I|^{2}+\overline{\operatorname{Ricc}}(\nu, \nu)\right) \\
& \geq m H^{2}+\inf _{M \backslash B_{R}} \overline{\operatorname{Ricc}}(\nu, \nu),
\end{aligned}
$$

where the last step follows from Newton inequality $|I I|^{2} \geq m H^{2}$. Letting $R \rightarrow+\infty$ we deduce the desired estimate for $H$.

REMARK 6.16. As observed in Theorem 5.23, if $M$ is a surface and $N^{3}$ has non-negative scalar curvature then $\operatorname{vol}(M)<+\infty$. Therefore, for $m=2$, the assumptions of Proposition 6.15 can be satisfied only when the scalar curvature of $N$ is somewhere negative.

In a similar fashion, Theorem 6.8 can be used to obtain information on the volume growth of the Martin-Morales-Nadirashvili minimal surface

$$
\varphi: M \rightarrow B_{1}(0) \subset \mathbb{R}^{3}
$$

introduced in Section 2.3. We recall a few preliminary facts to put the problem into perspective. It has been observed in [PRS05], Theorem 3.9 that $M$, being minimally immersed into a bounded region of $\mathbb{R}^{3}$, cannot be stochastically complete (see [Gri99] for a beautiful and detailed account on stochastic completeness). Since $M$ is complete, it follows from the sufficient condition in [Gri99], Theorem 9.1 that necessarily

$$
\frac{r}{\log \operatorname{vol}\left(B_{r}\right)} \in L^{1}(+\infty)
$$

In particular, $\operatorname{vol}(M)=+\infty$ and the growth of $\operatorname{vol}\left(B_{r}\right)$ is faster than $\exp \left\{a r^{2}\right\}$, for each $a>0$, at least along some divergent sequence $\left\{r_{k}\right\}$. However, to the best of our knowledge more precise lower bounds on $\operatorname{vol}\left(B_{r}\right)$ have still to be found. For instance, it is not clear whether $\operatorname{vol}\left(\partial B_{r}\right)$ can be bounded from above by some function

$$
f(r)=\Lambda \exp \left\{a r^{\alpha} \log ^{\beta} r\right\}
$$

for some suitable choices of $\Lambda, a>0, \alpha \geq 2$ and $\beta \geq 0$, or if $M$ has faster volume growth along some divergent sequence. We briefly describe here a possible way to get more information.

The basic step to prove the discreteness of the spectrum of the Martin-MoralesNadirashvili is inequality (2.52) of Theorem 2.25. In our setting, the manifold 
$Q$ reduces to a point, no $f$ appears, $k=0, \operatorname{sn}_{k}(r)=r, m=2, R_{0}=1$ and $\left|\mathrm{d}_{N} \varphi\right|^{2}=|\mathrm{d} \varphi|^{2}=2$. Hence, by (2.50), we can choose $c=2$ and (2.52) becomes

$$
\lambda_{1}^{-\Delta}\left(M \backslash \Omega_{R}\right) \geq \frac{2 R}{1-R^{2}}, \quad \text { where } \Omega_{R}=\{x \in M:|\varphi(x)|<R\} \Subset M .
$$

Suppose that we have a good knowledge of the links between $|\varphi(x)|$ and the intrinsic distance $r(x)$. For instance, suppose that we can provide a bound of the type $|\varphi(x)| \leq T(r(x))$, for some explicit, strictly increasing $T: \mathbb{R}^{+} \rightarrow(0,1)$ such that $T \rightarrow 1$ as $r \rightarrow+\infty$. Then, from $\Omega_{T(r)} \subset B_{r}$ and the monotonicity of eigenvalues we deduce that

$$
\frac{2 T(r)}{1-T^{2}(r)} \leq \lambda_{1}^{-\Delta}\left(M \backslash B_{r}\right) .
$$

Now, $M$ satisfies $1 / v \in L^{1}(+\infty)$, for otherwise by Corollary 6.10 we would have $\inf \sigma_{\text {ess }}(-\Delta)=0$, contradicting the fact that $M$ has discrete spectrum. Hence, Theorem 6.8 can be applied. If

$$
\operatorname{vol}\left(\partial B_{r}\right) \leq f(r)=\Lambda \exp \left\{a r^{\alpha} \log ^{\beta} r\right\} \quad \text { for some } \Lambda, a, \alpha \geq 2, \beta \geq 0,
$$

then we obtain

$$
\limsup _{r \rightarrow+\infty} \frac{2 T(r)}{\left[1-T^{2}(r)\right] r^{2(\alpha-1)} \log ^{2 \beta} r}<+\infty .
$$

This shows that a careful analysis of the growth of $2 T(r) /\left[1-T^{2}(r)\right]$ as a function of $r$ allows to deduce lower bounds on the growth of $\operatorname{vol}\left(\partial B_{r}\right)$, at least along a divergent sequence, that could possibly be faster than $r^{2}$. As a matter of fact, the above procedure can be carried on even for faster growths of type

$$
f(r)=\Lambda \exp \left\{a e^{b r}\right\}, \quad \Lambda, a b>0 .
$$

Indeed, $f$ as in (6.72) satisfies property $(P)$ of Definition 6.3 for every $c>1$. Thus, adapting the proof of Theorem 6.8, it can be shown that if $\operatorname{vol}(M)=+\infty$ and

$$
\operatorname{vol}\left(\partial B_{r}\right) \leq \Lambda \exp \left\{a e^{b r}\right\}, \quad \Lambda, a, b>0,
$$

then, for every $\mu>0$,

$$
\lim _{r \rightarrow+\infty}\left(\frac{\lambda_{1}^{-\Delta}\left(M \backslash B_{r}\right)}{\exp \{2 b(1+\mu) r\}}\right)=0 .
$$

However, the problem of finding an explicit $T(r)$ seems to be hard task. Nevertheless, maybe it could be more manageable than a direct estimate for $\operatorname{vol}\left(\partial B_{r}\right)$, mainly because of the technique employed to construct $M$.

Acknowledgements: the authors are deeply grateful to Proff. S. Pigola and A.G. Setti for having suggested them the paper [ER11], which leads to a definite improvement of the results of Section 5.4. Furthermore, they wish to express their gratitude to prof. M. L. Leite and to the referee for helpful comments and corrections, in particular regarding Section 5.5. 



\section{Bibliography}

[AC93] H. Alencar and M.P. Do Carmo, Hypersurfaces of constant mean curvature with finite index and volume of polynomial growth, Arch. Math. 60 (1993), no. 5, 489-493.

[AC98] H. Alencar and A.G. Colares, Integral formulas for the r-mean curvature linearized operator of a hypersurface, Ann. Global Anal. Geom. 16 (1998), 203-220.

[AF91] H. Alencar and K. Frensel, Hypersurfaces whose tangent geodesics omit a nonempty set, Pitman Monogr. Survey Pure Appl. Math. 52 (1991), 1-13.

[Agm85] S. Agmon, Bounds on exponential decay of eigenfunctions of Schrödinger operators, Schrdinger operators (Como, 1984), pp.1-38, Lecture Notes in Mathematics, vol. 1159, Springer, Berlin, 1985.

[Agm10] L Lectures on elliptic boundary value problems, Prepared for publication by B. Frank Jones, Jr. with the assistance of George W. Batten, Jr. Revised edition of the 1965 original, AMS Chelsea Publishing, Providence, RI, 2010.

[Ahl38] L.V. Ahlfors, An extension of Schwarz's lemma, Trans. Amer. Math. Soc. 43 (1938), 359-364.

[AK] K. Akutagawa and H. Kumura, The uncertainty principle lemma under gravity and the discrete spectrum of Schrödinger operators, available at arXiv:0812.4663.

[All74] W. Allegretto, On the equivalence of two types of oscillation for elliptic operators, Pacific J. Math. 55 (1974), 319-328.

[Amb57] W. Ambrose, A theorem of Myers, Duke Math. J. 24 (1957), 345-348.

[AO85] P. Avilès and R. Mc Owen, Conformal deformations of complete manifolds with negative curvature, J. Diff. Geom. 21 (1985), no. 2, 269-281.

[AO88] Conformal deformation to constant negative scalar curvature on noncompact Riemannian manifolds, J. Diff. Geom. 27 (1988), no. 2, 225-239.

[Aro57] N. Aronszajn, A unique continuation theorem for solution of elliptic partial differential equations or inequalities of second order., J. Math. Pures Appl. 36 (1957), 235-249.

[Bar37] J. Barta, Sur la vibration fundamentale d'une membrane, C. R. Acad. Sci. 204 (1937), $472-473$.

[BC97] J.L.M. Barbosa and A.G. Colares, Stability of Hypersurfaces with Constant r-Mean Curvature, Ann. Glob. An. Geom. 15 (1997), 277-297.

[BCS97] P. Bèrard, M.P. Do Carmo, and W. Santos, The index of constant mean curvature surfaces in hyperbolic 3-space, Math. Z. 234 (1997), 313-326.

[BJM10] G.P. Bessa, L.P. Jorge, and J.F. Montenegro, The spectrum of the Martin-MoralesNadirashvili minimal surface is discrete, J. Geom. Anal. 23 (2010), 63-71.

[BM07] G.P. Bessa and J.F. Montenegro, An extension of Barta's theorem and geometric applications, Ann. Global Anal. Geom. 31 (2007), no. 4, 345-362.

[BMR] B. Bianchini, L. Mari, and M. Rigoli, Yamabe-type equations with sign-changing nonlinearities on non-compact Riemannian manifolds, preprint.

[BMR09] _ Spectral radius, index estimates for Schrödinger operators and geometric applications, J. Funct. An. 256 (2009), 1769-1820.

[BN83] H. Brézis and L. Nirenberg, Positive solutions of nonlinear elliptic equations involving critical Sobolev exponents, Comm. Pure Appl. Math. 36 (1983), no. 4, 437-477.

[BR97] B. Bianchini and M. Rigoli, Non existence and uniqueness of positive solutions of Yamabe type equations on non positively curved manifolds, Trans. Amer. Math. Soc. 349 (1997), 4753-4774.

[Bro81] R. Brooks, A relation between growth and the spectrum of the Laplacian, Math. Z. 178 (1981), no. 4, 501-508. 
[Bro84] _ On the spectrum of noncompact manifolds with finite volume, Math. Z. 187 (1984), no. 3, 425-432.

[BRS98a] L. Brandolini, M. Rigoli, and A.G. Setti, Positive Solutions of Yamabe Type Equations on Complete Manifolds and Applications, J. Funct. An. 160 (1998), 176-222.

[BRS98b] _ Positive solutions of Yamabe-type equations on the Heisenberg group, Duke Math. J. 91 (1998), no. 2, 241-296.

[Cal67] E. Calabi, On Ricci curvature and geodesics, Duke Math. J. 34 (1967), 667-676.

[Car92] M.P. Do Carmo, Riemannian Geometry, Mathematics: Theory and Applications, Birkäuser Boston INC, Boston, MA, 1992.

[Cas06] P. Castillon, An inverse spectral problem on surfaces, Comm. Math. Helv. 81 (2006), 271-286.

[CGT82] J. Cheeger, M. Gromov, and M. Taylor, Finite propagation speed, Kernel estimates for functions of the Laplace operator and the geometry of complete Riemannian manifolds, J. Diff. Geom. 17 (1982), 15-53.

[Cha84] I. Chavel, Eigenvalues in Riemannian Geometry, Pure and Applied Mathematics, 115, Academic Press, Inc., Orlando, FL, 1984.

[Che70] J. Cheeger, A lower bound for the smallest eigenvalue of the Laplacian, Problems in analysis (Papers dedicated to Salomon Bochner, 1969. Princeton Univ. Press, Princeton, N. J.) (1970), 195-199.

[Che73] P. Chernoff, Essential self-adjointness of powers of generators of hyperbolic equations, J. Funct. An. 12 (1973), 401-414.

[CL87] K.S. Cheng and J.T. Lin, On the elliptic equations $\Delta u=K(x) u^{\sigma}$ and $\Delta u=K(x) e^{2 u}$, Trans. Amer. Math. Soc. 304 (1987), no. 2, 639-668.

[CM02] T. Colding and W. Minicozzi, Estimates for parametric elliptic integrands., Int. Math. Res. Not. (2002), no. 6, 291-297.

[CM05] , The Calabi-Yau conjectures for embedded surfaces, Ann. Math. 161 (2005), $727-758$.

[CM11] A. Cianchi and V. Mazýa, On the discreteness of the spectrum of the Laplacian on noncompact Riemannian manifolds, J. Diff. Geom. 87 (2011), no. 3, 469-491.

[CP79] M.P. Do Carmo and C.K. Peng, Stable complete minimal surfaces in $\mathbb{R}^{3}$ are planes, Bull. Amer. Math. Soc. 1 (1979), 903-906.

[CR] M. Castelpietra and L. Rifford, Regularity properties of the distance functions to conjugate and cut loci for viscosity solutions of Hamilton-Jacobi equations and applications in Riemannian geometry, ESAIM Control Optim. Calc. Var. 16, no. 3, 695-718.

[CV35] S. Cohn-Vossen, KürzesteWege und Totalkrümmung auf Flächen. (German), Compositio Math. 2 (1935), 69-133.

[CY75] S.Y. Cheng and S.T. Yau, Differential equations on Riemannian manifolds and their geometric applications, Comm. Pure Appl. Math. 28 (1975), no. 3, 333-354.

[CZ99] M.P. Do Carmo and D. Zhou, Eigenvalue estimate on complete non compact Riemannian manifolds and applications, Trans. Amer. Math. Soc. 351 (1999), 1391-1401.

[Dav95] E.B. Davies, Spectral theory and differential operators, Cambridge University Press, 1995.

[Dev] B. Devyver, On the finiteness of the Morse Index for Schrödinger operators, to appear on Manuscr. Math., first version available at arXiv:1011.3390.

[DG92] H. Donnelly and N. Garofalo, Riemannian manifolds whose Laplacians have purely continuous spectrum, Math. Ann. 293 (1992), 143-161.

[DG97] Schrödinger operators on manifolds, essential self-adjointness, and absence of eigenvalues, J. Geom. Anal. 7 (1997), no. 2, 241-257.

[DN85] W.Y. Ding and W.M. Ni, On the elliptic equation $\Delta u+K u^{(n+2) /(n-2)}=0$ and related topics, Duke Math. J. 52 (1985), no. 2, 485-506.

[Don81a] H. Donnelly, Eigenvalues embedded in the continuum for negatively curved manifolds, Michigan Math. J. 28 (1981), 53-62.

[Don81b] _ On the essential spectrum of a complete Riemannian manifold, Topology 20 (1981), no. 1, 1-14

[Don90] - Negative curvature and embedded eigenvalues, Math. Z. 203 (1990), 301-308.

[EF92] J.F. Escobar and A. Freire, The spectrum of the Laplacian on manifolds of positive curvature, Duke Math. J. 65 (1992), 1-21. 
[EFK11] T. Ekholm, R.L. Frank, and H. Kovař̀k, Eigenvalue estimates for Schroedinger operators on metric trees, Adv. Math. 226 (2011), no. 6, 5165-5197.

[EG92] L.C. Evans and R.F. Gariepy, Measure theory and fine properties of functions, Studies in Advanced Mathematics, CRC Press, Boca Raton, FL, 1992.

[EH90] J.H. Eschenburg and E. Heintze, Comparison theory for Riccati equations, Manuscripta Math. 68 (1990), 209-214.

[EL86] W.D. Evans and T. Lewis, Eigenvalues below the essential spectra of singular elliptic operators, Trans. Amer. Math. Soc. 297 (1986), no. 1, 197-222.

[Elb02] M.F. Elbert, Constant positive 2-mean curvature hypersurfaces, Illinois J. Math. 46 (2002), 247-267.

[EO80] J.H. Eschenburg and J.J. O'Sullivan, Jacobi tensors and ricci curvature, Math. Ann. 252 (1980), 1-26.

[ER91] K.D. Elworthy and S. Rosenberg, Manifolds with wells of negative curvature, Invent. Math. 103 (1991), no. 3, 471-495, With an appendix by Daniel Ruberman.

[ER11] J.M. Espinar and H. Rosenberg, A Colding-Minicozzi stability inequality and its applications, Trans. Amer. Math. Soc. 363 (2011), no. 5, 2447-2465.

[Esc85] J. Escobar, On the spectrum of the Laplacian on complete Riemannian manifolds, Commun. Partial Diff. Eq. 11 (1985), 63-85.

[FC85] D. Fischer-Colbrie, On complete minimal surfaces with finite Morse index in three manifolds, Invent. Math. 82 (1985), 121-132.

[FCS80] D. Fischer-Colbrie and R. Schoen, The structure of complete stable minimal surfaces in 3-manifolds of non negative scalar curvature, Comm. Pure Appl. Math. XXXIII (1980), 199-211.

[Fed69] H. Federer, Geometric Measure Theory, Springer-Verlag, New York, 1969.

[Fia41] F. Fiala, Le problème des isopérimètres sur les surfaces ouvertes à courbure positive, Comm. Math. Helv. 13 (1941), 293-346.

[Fin65] R. Finn, On a class of conformal metrics, with application to differential geometry in the large, Comm. Math. Helv. 40 (1965), 1-30.

[Fit18] W.B. Fite, Concerning the zeros of the solutions of certain differential equations, Trans. Amer. Math. Soc. 19 (1918), 341-352.

[Ğ̈9] L. Gärding, An inequality for hyperbolic polynomials., J. Math. Mec. 8 (1959), 957-965.

[Gaf54] M.P. Gaffney, A special Stokes's theorem for complete Riemannian manifolds, Ann. Math. 60 (1954), no. 2, 140-145.

[Gag80] M.E. Gage, Upper bounds for the first eigenvalue of the Laplace-Beltrami operator, Indiana Univ. Math. J. 29 (1980), no. 6, 897-912.

[Gal82] G.J. Galloway, Compactness criteria for Riemannian manifolds, Proc. Amer. Math. Soc. 84 (1982), 106-110.

[GHL90] S. Gallot, D. Hulin, and J. Lafontaine, Riemannian Geometry, Springer-Verlag, Berlin, 1990.

[Gla65] I.M. Glazman, Direct Methods of Qualitative Spectral Analysis of Singular Differential Operators, Israel Program for Scientific Translations, Davey, Hartford, Conn., 1965.

[GP94] R. Grimaldi and P. Pansu, Sur la régularité de la fonction croissance d'une variété riemannienne, Geom. Dedicata 50 (1994), no. 3, 301-307.

[Gri99] A. Grigor'yan, Analytic and geometric background of recurrence and non-explosion of the Brownian motion on Riemannian manifolds, Bull. Amer. Math. Soc. 36 (1999), 135-249.

[GS77] K. Grove and K. Shiohama, A generalized sphere theorem, Ann. Math. 106 (1977), no. 2, 201-211.

[GT98] D. Gilbarg and N. Trudinger, Elliptic Partial Differential Equations of Second Order, third ed., Springer-Verlag, 1998.

[Gui92] F.F. Guimarães, The integral of the scalar curvature of complete manifolds without conjugate points, J. Diff. Geom. 36 (1992), no. 3, 651-662.

[Gul86] R. Gulliver, Index and total curvature of complete minimal surfaces, Geom. Meas. Theory and Calc. Var. (Arcata, Calif., 1984), Proc. Sympos. Pure Math. 44 (1986), 207-211.

[Gul88] Minimal surfaces of finite index in manifolds of positive scalar curvature, Calculus of Variations and Partial Differential Equations (Trento), Lect. Notes Math., vol. 1340, Springer, 1988. 
[GW79] R.E. Greene and H. Wu, Function theory on manifolds which possess a pole, Lecture Notes in Mathematics, vol. 699, Springer, Berlin, 1979.

[Hal71] B. Halpern, On the immersion of an m-dimensional manifold in $(m+1)$-dimensional Euclidean space, Proc. Am. Math. Soc. 30 (1971), 181-184.

[Har64a] P. Hartman, Geodesic parallel coordinates in the large, Amer. J. Math. 86 (1964), $705-727$.

[Har64b] _ Ordinary Differential Equations, John Wiley, 1964.

[Hig01] Y. Higuchi, A remark on exponential growth and the spectrum of the Laplacian, Kodai Math. J. 24 (2001), 42-47.

[Hil48] E. Hille, Non-oscillation theorems, Trans. Amer. Math. Soc. 64 (1948), 234-252.

[HK84] T. Hasanis and D. Koutroufiotis, A property of complete minimal surfaces, Trans. Am. Math. Soc. 281 (1984), 833-843.

[HL95] J. Hounie and M.L. Leite, The maximum principle for hypersurfaces with vanishing curvature functions, J. Diff. Geom. 41 (1995), 247-258.

[HL99] $\quad$ Two-ended hypersurfaces with zero scalar curvature, Indiana Univ. Math. J. 48 (1999), no. 3, 867-882.

[HLP52] G.H. Hardy, J.E. Littlewood, and G. Polya, Inequalities (2d ed.), Cambridge University Press, 1952.

[Hub57] A. Huber, On subharmonic functions and differential geometry in the large, Comm. Math. Helv. 32 (1957), 13-72.

[IMR11] D. Impera, L. Mari, and M. Rigoli, Some geometric properties of hypersurfaces with constant r-mean curvature in Euclidean space, Proc. Amer. Math. Soc. 139 (2011), no. $6,2207-2215$.

[IT98] J.I. Itoh and M. Tanaka, The dimension of a cut locus on a smooth Riemannian manifold, Tôhoku Math. J. 50 (1998), 571-575.

[IT01a] - The Lipschitz continuity of the distance function to the cut locus, Trans. Amer. Math. Soc. 353 (2001), no. 1, 21-40.

[IT01b] _ A Sard Theorem for the distance function, Math. Ann. 320 (2001), 1-10.

[Kat74] T. Kato, A second look at the essential selfadjointness of the Schrödinger operators, Physical reality and mathematical description. Reidel, Dodrecht (1974), 193-201.

[Kat95] _ Perturbation theory for linear operators, Reprint of the 1980 edition. Classics in Mathematics, Springer-Verlag, Berlin, 1995.

[Kaz85] J.L. Kazdan, Prescribing the Curvature of a Riemannian Manifold, CBMS Regional Conference Series in Mathematics, vol. 57, Published for the Conference Board of the Mathematical Sciences, Washington, DC, by the American Mathematical Society, Providence, RI, 1985.

[KF80] A.N. Kolmogorov and S.V. Fomin, Elements of function theory and functional analysis, (Italian translation), Mir, 1980.

[Kle88] R. Kleine, Discreteness conditions for the Laplacian on complete, noncompact Riemannian manifolds, Math. Z. 198 (1988), no. 1, 127-141.

[KN84] C. Kenig and W.M. Ni, An exterior Dirichlet problem with applications to some nonlinear equations arising in geometry, Amer. J. Math. 106 (1984), no. 3, 689-702.

[KS88] W. Kirsch and B. Simon, Corrections to the classical behavior of the number of bound states of Schrödinger operators, Ann. Phys. 183 (1988), 122-130.

[Kum] H. Kumura, A note on the best constant of decay of eigenfunctions on Riemannian manifolds, available at arXiv:1108.0034.

[Kum97] _ On the essential spectrum of the Laplacian on complete manifolds, J. Mat. Soc. Japan 49 (1997), no. 1, 1-14.

[Kum02]__ A note on the absence of eigenvalues on negatively curved manifolds, Kyushu J. Math. 56 (2002), 109-121.

[Kum05] _ On the essential spectrum of the Laplacian and vague convergence of the curvature at infinity, Comm. P. Diff. Eq. 30 (2005), no. 10-12, 1555-1565.

[Kum11] _ The lower bound of the Ricci curvature that yields the infinite number of the discrete spectrum of the Laplacian, Ann. de l'inst. Fourier 61 (2011), no. 4, 1557-1572.

[Kup86] D.N. Kupeli, On existence and comparison of conjugate points in Riemannian and lorentzian geometry, Math. Ann. 276 (1986), no. 1, 67-79.

[Kur89] T. Kura, The weak supersolution-subsolution method for second order quasilinear elliptic equations, Hiroshima Math. J. 19 (1989), no. 1, 1-36. 
[LD79] P. Li and H. Donnelly, Pure point spectrum and negative curvature for noncompact manifolds, Duke Math. J. 46 (1979), no. 3, 497-503.

[Leb72] N. N. Lebedev, Special Functions and Their Applications, Dover N.Y., 1972.

[Lei50] W. Leighton, The detection of the oscillation of solutions of a second order linear differential equation, Duke Math. J. 17 (1950), 57-61.

[Li94] J. Li, Spectrum of the Laplacian on a complete Riemannian manifold with non-negative ricci curvature which possess(es) a pole, J. Math. Soc. Japan 46 (1994), 213-216.

[Lin85] F.H. Lin, On the elliptic equation $D_{i}\left[a_{i j}(x) D_{j} U\right]-k(x) U+K(x) U^{p}=0$, Proc. Amer. Math. Soc. 95 (1985), no. 2, 219-226.

[LN88] Y. Li and W.M. Ni, On conformal scalar curvature equations in $\mathbb{R}^{n}$, Duke Math. J. 57 (1988), no. 3, 895-924.

[LN05] Y. Li and L. Nirenberg, The distance function to the boundary, Finsler geometry, and the singular set of viscosity solutions of some Hamilton-Jacobi equations, Comm. Pure Appl. Math. 58 (2005), no. 1, 85-146.

[LS84] P. Li and R. Schoen, $L^{p}$ and mean value properties of subharmonic functions on Riemannian manifolds, Acta Math. 153 (1984), no. 3-4, 279-301.

[LTD98] P. Li, L.F. Tam, and D.Yang, On the elliptic equation $\Delta u+k u-K u^{p}=0$ on complete Riemannian manifolds and their geometric applications, Trans. Amer. Math. Soc. 350 (1998), no. 3, 1045-1078.

[LW06] P. Li and J. Wang, Weighted Poincaré inequality and rigidity of complete manifolds, Ann. Sci. École Norm. Sup. (4) 39 (2006), no. 6, 921-982.

[Mar] L. Mari, On the equivalence of finite Morse index and stability at infinity for Schrödinger operators on Riemannian manifolds, submitted.

[McK70] H.P. McKean, An upper bound to the spectrum of $\Delta$ on a manifold of negative curvature, J. Diff. Geom. 4 (1970), 359-366.

[MM03] C. Mantegazza and A.C. Mennucci, Hamilton-Jacobi equations and distance functions on Riemannian manifolds, Appl. Math. Optim. 47 (2003), 1-25.

[MM05] F. Martin and S. Morales, Complete proper minimal surfaces in convex bodies of $\mathbb{R}^{3}$, Duke Math. J. 128 (2005), 559-593.

[MM06] Complete proper minimal surfaces in convex bodies of $\mathbb{R}^{3}$. II. The behaviour of the limit set, Comment. Math. Helv. 81 (2006), 699-725.

[Moo55] R.A. Moore, The behavior of solutions of a linear differential equation of second order, Pacific J. Math. 5 (1955), 125-145.

[MP78] W.F. Moss and J. Piepenbrink, Positive solutions of elliptic equations, Pac. J. Math. 75 (1978), 219-226.

[MPR08] W. Meeks, J. Perez, and A. Ros, Stable constant mean curvature hypersurfaces, Handbook of Geometric Analysis. International Press 1 (2008), 301-380.

[MRS10] L. Mari, M. Rigoli, and A.G. Setti, Keller-Osserman conditions for diffusion-type operators on Riemannian manifolds, J. Funct. An. 258 (2010), no. 2, 665-712.

[MRV] P. Mastrolia, M. Rimoldi, and G. Veronelli, Myers' type theorems and some related oscillation results, to appear on J. Geom. Anal.

[Mye41] S.B. Myers, Riemannian manifolds with positive mean curvature, Duke Math. J. 8 (1941), 401-404.

[Nad96] N. Nadirashvili, Hadamard's and Calabi-Yau's conjecture on negatively curved and minimal surfaces, Invent. Math. 126 (1996), 457-465.

[Nai84] M. Naito, A note on bounded positive entire solutions of semilinear elliptic equations, Hiroshima Math. J. 14 (1984), 211-214.

[Neh57] Z. Nehari, Oscillation criteria for second-order linear differential equations, Trans. Amer. Math. Soc. 85 (1957), 428-445.

[Ni82] W.M. Ni, On the Elliptic Equation $\Delta u+K(x) u^{(n+2) /(n-2)}=0$, its Generalizations, and Applications in Geometry, Indiana Univ. Math. J. 31 (1982), no. 4, 493-529.

[OK90] B. Opic and A. Kufner, Hardy inequalities, Pitman Research Notes in Mathematics, vol. 219, Longman Scientific \& Technical, Harlow, 1990.

[Ozo74] V. Ozols, Cut loci in Riemannian manifolds, Tôhoku Math. J. 26 (1974), 219-227.

[Per60] A. Persson, Bounds for the discrete part of the spectrum of a semi-bounded Schrödinger operator, Math. Scand. 8 (1960), 143-153.

[Pet97] P. Petersen, Riemannian Geometry, Springer-Verlag, 1997. 
[Pie74a] J. Piepenbrink, Finiteness of the Lower Spectrum of Schrödinger Operators, Math. Z. 140 (1974), 29-40.

[Pie74b] - Nonoscillatory Elliptic Equations, J. Diff. Eq. 15 (1974), 541-550.

[Pie77]__ A Conjecture of Glazman, J. Diff. Eq. 24 (1977), 173-177.

[Pin78] M.A. Pinsky, The spectrum of the Laplacian on a manifold of negative curvature I, J. Diff. Geom. 13 (1978), 87-91.

[Pin81]_ On the spectrum of Cartan-Hadamard manifolds, Pacific J. Math. 94 (1981), no. $1,223-230$.

[Pog81] A.V. Pogorelov, On the stability of minimal surfaces, Soviet Math. Dokl. 24 (1981), 274-276.

[PRRS11] S. Pigola, M. Rimoldi, M. Rigoli, and A.G. Setti, Ricci almost solitons, Ann. Sc. Norm. Sup. Pisa X (2011), no. 4, 757-799.

[PRS05] S. Pigola, M. Rigoli, and A.G. Setti, Maximum principles on Riemannian manifolds and applications, Mem. Amer. Math. Soc. 174 (2005), no.822.

[PRS06] Maximum principles at infinity on Riemannian manifolds: an overview, Mat. Contemp. 31 (2006), 81-128.

[PRS08] _ Vanishing and finiteness results in Geometric Analisis. A generalization of the Böchner technique, Progress in Math., vol. 266, Birkäuser, 2008.

[PRS10] _ Existence and non-existence results for a logistic-type equation on manifolds, Trans. Amer. Math. Soc. 362 (2010), no. 4, 1907-1936.

[PW84] M.H. Protter and H.F. Weinberger, Maximum principles in differential equations, (Corrected reprint of the 1967 original), Springer-Verlag, 1984.

[PW97] P. Petersen and G. Wei, Relative volume comparison with integral curvature bounds, Geom. Funct. Anal. 7 (1997), 1031-1045.

[Rei73] R.C. Reilly, Variational properties of functions of the mean curvatures for hypersurfaces in space forms, J. Diff. Geom. 8 (1973), no. 3, 465-477.

[Rif04] L. Rifford, A Morse-Sard theorem for the distance function on Riemannian manifolds, Manuscripta Math. 113 (2004), 251-265.

[Ros93] H. Rosenberg, Hypersurfaces of constant curvature in space forms, Bull. Sc. Math., $2^{e}$ Série 117 (1993), 211-239.

[RRV94] A. Ratto, M. Rigoli, and L. Veron, Scalar curvature and conformal deformation of hyperbolic space, J. Funct. An. 121 (1994), no. 1, 15-77.

[RRV97] - Scalar curvature and conformal deformations of noncompact Riemannian manifolds, Math. Z. 225 (1997), 395-426.

[RS75] M. Reed and B. Simon, Methods of Modern Mathematical Physics. II. Fourier Analysis, Self Adjointness, Academic Press, New York-London, 1975.

[RS78] Methods of Modern Mathematical Physics. IV. Analysis of Operators, Academic Press, New York-London, 1978.

[RS80] _ Methods of Modern Mathematical Physics. I. Functional Analysis (2nd ed.), Academic Press, New York, 1980.

[RS01] M. Rigoli and A.G. Setti, On the $L^{2}$-form spectrum of the Laplacian on nonnegatively curved manifolds, Tôhoku Math. J. 53 (2001), 443-452.

[RY94] S. Rosenberg and D. Yang, Bounds on the fundamental group of a manifold with almost nonnegative Ricci curvature, J. Math. Soc. Japan 46 (1994), no. 2, 267-287.

[Sak96] T. Sakai, Riemannian Geometry, Translations of Mathematical Monographs, vol. 149, American Mathematical Society, Providence, RI, 1996.

[Sar58] A. Sard, Images of Critical Sets, Ann. Math. 68 (1958), no. 2, 247-259.

[Sat72] D.H. Sattinger, Monotone methods in nonlinear elliptic and parabolic boundary value problems, Indiana Univ. Math. J. 21 (1971/72), 979-1000.

[Shi84] K. Shiohama, The role of Total Curvature on complete noncompact Riemannian 2manifolds, Ill. J. Math. 28 (1984), no. 4, 597-620.

[Shi85] - Total curvatures and Minimal Areas of Complete Open Surfaces, Proc. Amer. Math. Soc. 94 (1985), no. 2, 310-316.

[Sma65] S. Smale, On the Morse index theorem, J. Math. Mech. 14 (1965), 1049-1055.

[Sma67]_ On the Morse index theorem (corrigendum), J. Math. Mech. 16 (1967), 10691970.

[ST93] K. Shiohama and M. Tanaka, The length function of geodesic parallel circles, Progress in Diff. Geom., Adv. Stud. Pure Math. 22 (1993), 299-308. 
[Ste70] W. Stenger, On two complementary variational characterizations of eigenvalues, Inequalities, II (Proc. Second Sympos., U.S. Air Force Acad., Colo., 1967, Academic Press, New York, 1970.

[Str83] R.S. Strichartz, Analysis of the Laplacian on the complete Riemannian manifold, J. Funct. An. 52 (1983), no. 1, 48-79.

[Swa68] C.A. Swanson, Comparison and Oscillation Theory for Linear differential operators, Academic Press, New York and London, 1968.

[Tay89] M.E. Taylor, $L^{p}$-estimates on functions of the Laplace operator, Duke Math. J. 58 (1989), no. 3, 773-793.

[Tol84] P. Tolksdorf, Regularity of a more general class of quasilinear elliptic equations, J. Diff. Eq. 51 (1984), 126-150.

[War67] F.W. Warner, Conjugate loci of constant order, Ann. Math. 86 (1967), no. 1, 192-212.

[Wei87] J. Weidmann, Spectral theory of ordinary differential operators, Lecture Notes in Mathematics, vol. 1258, Springer-Verlag, Berlin, 1987.

[Wer68] A.L. Werner, Concerning S. Cohn-Vossen's theorem on the integral curvature of complete surfaces, Siber. Math. J. 9 (1968), 150-153.

[Win49] A. Wintner, A criterion of oscillatory stability, Quart. Appl. Math. 7 (1949), 115-117.

[Wu91] J.Y. Wu, Complete manifolds with a little negative curvature, Amer. J. Math. 113 (1991), no. 4, 567-572.

[Yau76] S.T. Yau, Some function-theoretic properties of complete Riemannian manifold and their applications to geometry, Indiana Univ. Math. J. 25 (1976), no. 7, 659-670.

[Yau00] Review of Geometry and Analysis, Asian J. Math. 4 (2000), 235-278.

[YS79] S.T. Yau and R. Schoen, Existence of incompressible minimal surfaces and the topology of three dimensional manifolds with non-negative scalar curvature, Ann. Math. 110 (1979), 127-142.

[Zha04] Q.S. Zhang, Positive solutions to $\Delta u-V u+W u^{p}=0$ and its parabolic counterpart in noncompact manifolds, Pacific J. Math. 213 (2004), no. 1, 163-200. 